
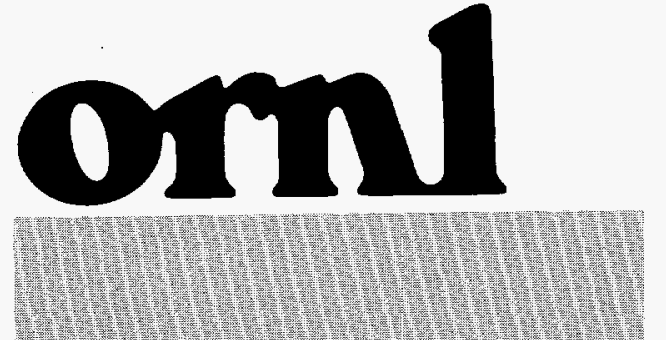

OAK RIDGE

NATIONAL

LABORATORY

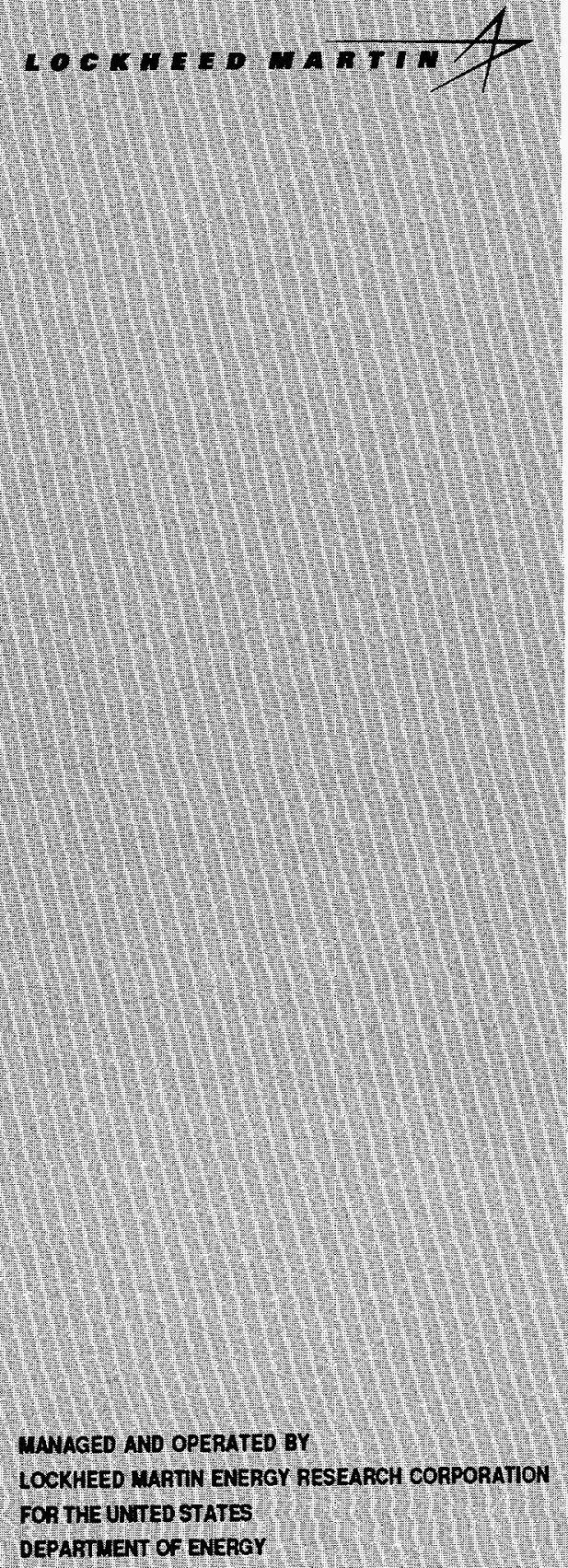

\section{CFIVED \\ JUL 151908 \\ OSTI}

\section{Nuclear and Fundamental Physics Instrumentation for the ANS Project}

S. J. Robinson

S. Raman

J. Arterburn

T. McManamy

F. J. Peretz

H. Faust

A. E. Piotrowski

Completed: August 1995

Published: May 1996

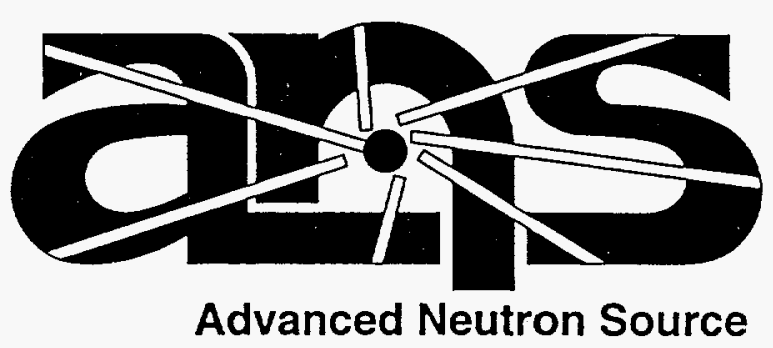


This report has been reproduced directly from the best available copy.

Available to DOE and DOE contractors from the Office of Scientific and Technical Information, P.O. Box 62, Oak Ridge, TN 37831; prices available from (615) 576-8401, FTS 626-8401.

Available to the public from the National Technical Information Service, U.S. Department of Commerce, 5285 Port Royal Rd., Springfield, VA 22161.

This report was prepared as an account of work sponsored by an agency of the United States Government. Neither the United States Government nor any agency thereof, nor any of their employees, makes any warranty, express or implied, or assumes any legal liability or responsibility for the accuracy, completeness, or usefulness of any information, apparatus, product, or process disclosed, or represents that its use would not infringe privately owned rights. Reference herein to any specific commercial product, process, or service by trade name, trademark, manufacturer, or otherwise, does not necessarily constitute or imply its endorsement, recommendation, or favoring by the United States Government or any agency thereof. The views and opinions of authors expressed herein do not necessarily state or reflect those of the United States Government or any agency thereot. 


\title{
NUCLEAR AND FUNDAMENTAL PHYSICS INSTRUMENTATION FOR THE ANS PROJECT
}

\author{
S. J. Robinson \\ Physics Department, Tennessee Technological University \\ Cookeville, Tennessee 38505 \\ S. Raman \\ Physics Division, Oak Ridge National Laboratory \\ Oak Ridge, Tennessee 37831 \\ J. Arterburn, T. McManamy, and F.J. Peretz \\ ANS Project, Oak Ridge National Laboratory \\ Oak Ridge, Tennessee 37831 \\ H. Faust \\ Institut Laue-Langevin \\ Grenoble, France \\ A. E. Piotrowski \\ Soltan Institute for Nuclear Studies \\ 0S-400, Swierk, Poland \\ Completed: August 1995 \\ Published: May 1996 \\ Prepared by \\ OAK RIDGE NATIONAL LABORATORY \\ Oak Ridge, Tennessee 37831 \\ managed by \\ LOCKHEED MARTIN ENERGY RESEARCH CORP. \\ for the \\ U.S. DEPARTMENT OF ENERGY \\ under contract DE-AC05-96OR22464
}





\section{DISCLAIMER}

Portions of this document may be illegible in electronic image products. Images are produced from the best available original document. 



\section{CONTENTS}

LIST OF FIGURES $\ldots \ldots \ldots \ldots \ldots \ldots \ldots \ldots \ldots \ldots \ldots \ldots \ldots \ldots \ldots \ldots$

ACRONYMS $\ldots \ldots \ldots \ldots \ldots \ldots \ldots \ldots \ldots \ldots \ldots \ldots \ldots \ldots \ldots \ldots \ldots$

PREFACE $\ldots \ldots \ldots \ldots \ldots \ldots \ldots \ldots \ldots \ldots \ldots \ldots \ldots \ldots \ldots \ldots \ldots \ldots \ldots \ldots$

1. INTRODUCTION $\ldots \ldots \ldots \ldots \ldots \ldots \ldots \ldots \ldots \ldots \ldots \ldots \ldots \ldots \ldots \ldots$

2. DEFINITION OF INSTRUMENT SYSTEMS $\ldots \ldots \ldots \ldots \ldots \ldots \ldots \ldots \ldots \ldots \ldots \ldots$

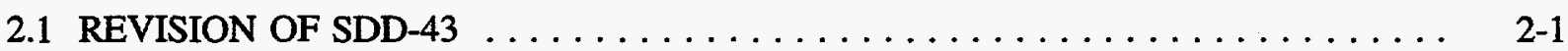

2.2 PRELIMINARY BUDGETS FOR INSTRUMENT CONSTRUCTION $\ldots \ldots \ldots \ldots \ldots$ 2-1

3. DESIGN STUDIES FOR THE ON-LINE ISOTOPE SEPARATION FACILITY . . . . . 3-1

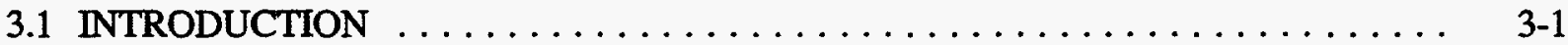

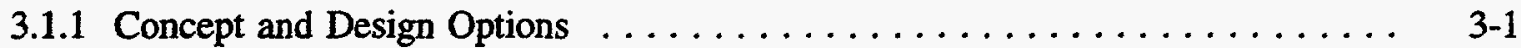

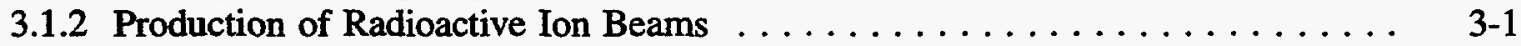

3.2 ION SOURCES-DETAILED DESIGN OPTIONS $\ldots \ldots \ldots \ldots \ldots \ldots \ldots \ldots \ldots . \ldots$

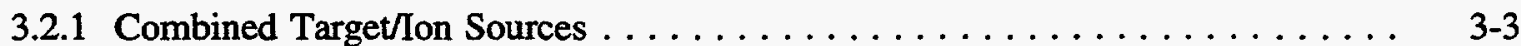

3.3 INTERNAL TARGET AND HELIUM-JET ION SOURCE $\ldots \ldots \ldots \ldots \ldots \ldots \ldots$

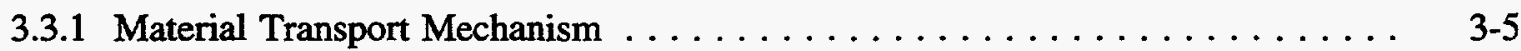

3.3.2 Helium Transport System . . . . . . . . . . . . . . . . . . . 3-14

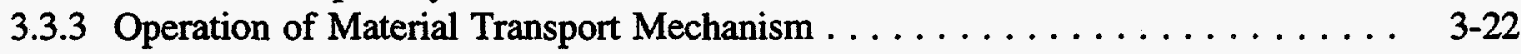

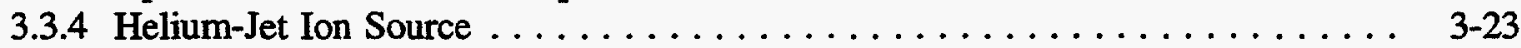

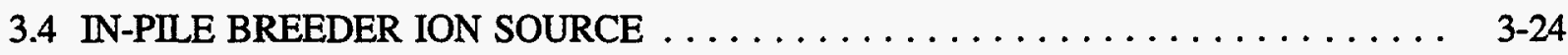

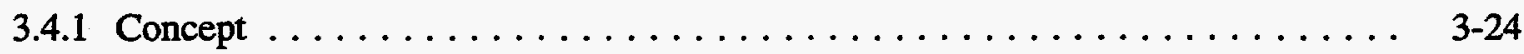

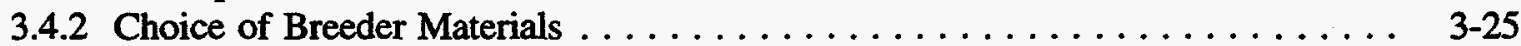

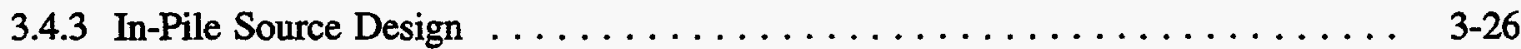

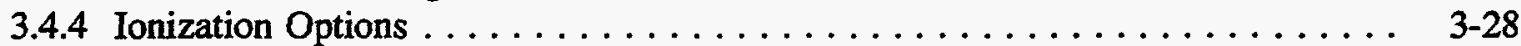

3.4.5 Beam Transport in Pile . . . . . . . . . . . . . . . . . . . . . . . . 3-29

3.4.6 Bending Magnet and First Mass Separation . . . . . . . . . . . . . . 3-29

3.4 .7 Parasitic Uses . . . . . . . . . . . . . . . . . . . . . .

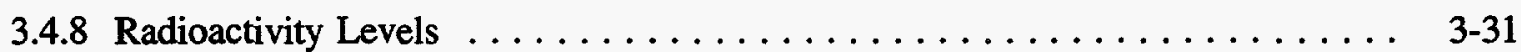

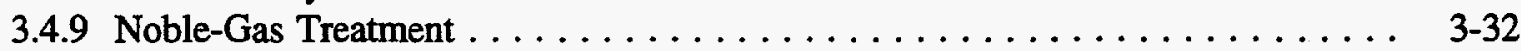

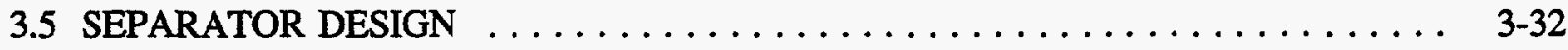

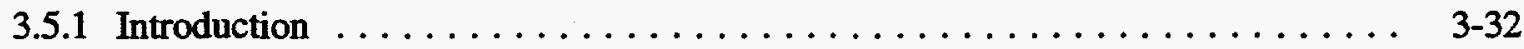

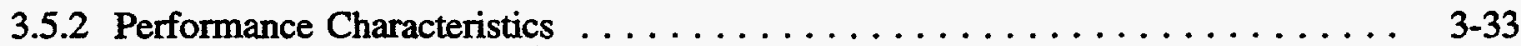

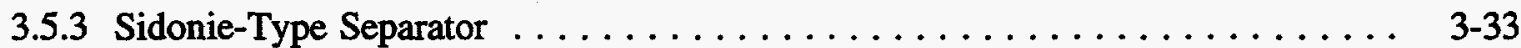

3.5.4 High Mass-Resolving-Power Separator . . . . . . . . . . . . . 3-35

3.5.5 Focal-Plane Box, Switchyard, and Beam Lines . . . . . . . . . . . . 3-35

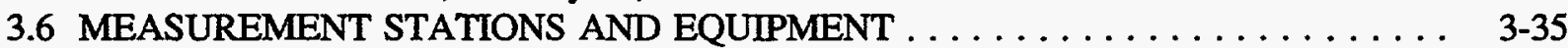

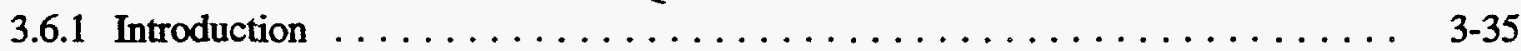

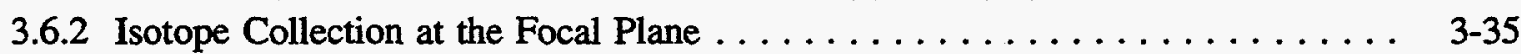

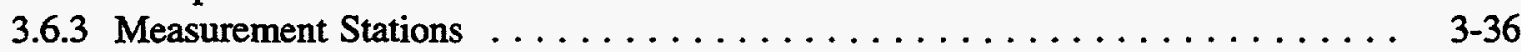

3.6 .4 Tape Transport Systems . . . . . . . . . . . . . . . . . . 3-37

3.7 APPLICATION FOR RADIOACTIVE ION BEAMS $\ldots \ldots \ldots \ldots \ldots \ldots \ldots \ldots \ldots$ 


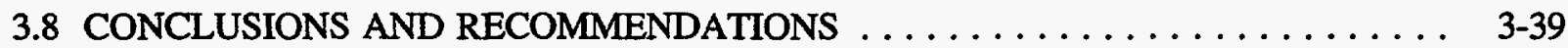

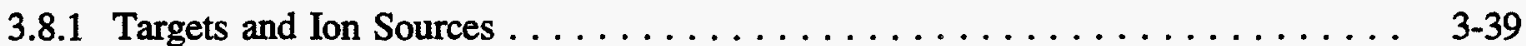

3.8.2 Separator Design . . . . . . . . . . . . . . . . . . . . . . 39

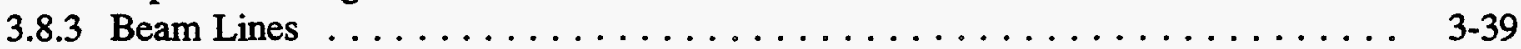

4. DESIGN CONSIDERATIONS FOR THE THROUGH-TUBE $\ldots \ldots \ldots \ldots \ldots \ldots \ldots \ldots$ 4-1

4.1 SOURCE-HOLDER ASSEMBLY DESIGN $\ldots \ldots \ldots \ldots \ldots \ldots \ldots \ldots \ldots \ldots$. . . . . . . .

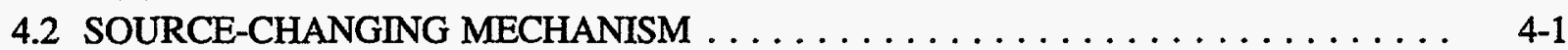

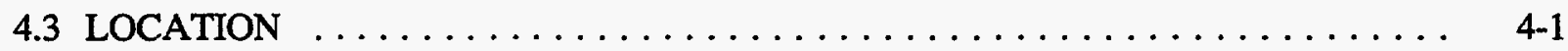

5. DETECTORS FOR NUCLEAR SPECTROSCOPY $\ldots \ldots \ldots \ldots \ldots \ldots \ldots \ldots \ldots \ldots$ 5-1

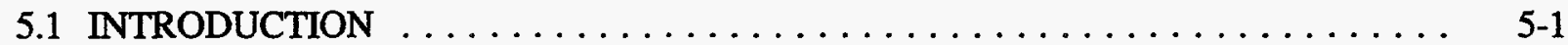

5.2 NEED FOR DETECTORS AT ANS $\ldots \ldots \ldots \ldots \ldots \ldots \ldots \ldots \ldots \ldots \ldots$

5.3 GENERAL DETECTOR POOL $\ldots \ldots \ldots \ldots \ldots \ldots \ldots \ldots \ldots \ldots \ldots \ldots \ldots$

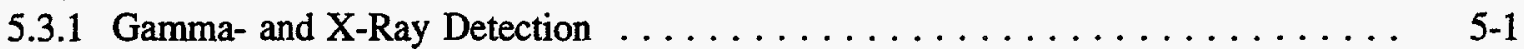

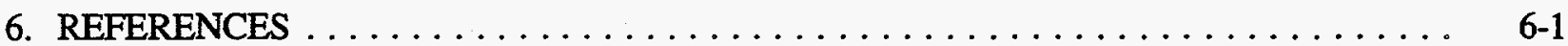

Appendix A. JUNE 1993 SDD-43 $\ldots \ldots \ldots \ldots \ldots \ldots \ldots \ldots \ldots \ldots \ldots \ldots \ldots \ldots$

Appendix B. COST AND PERSONNEL ESTIMATES $\ldots \ldots \ldots \ldots \ldots \ldots \ldots \ldots$

Appendix C. CONVENTIONAL ION SOURCE TYPES $\ldots \ldots \ldots \ldots \ldots \ldots \ldots \ldots$ C-1 


\section{LIST OF FIGURES}

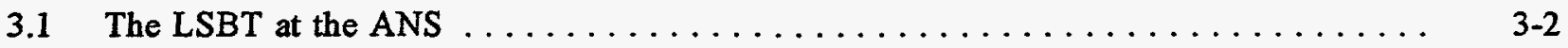

3.2 The main elements of the MTM in relation to the LSBT $\ldots \ldots \ldots \ldots \ldots \ldots$

3.3 Front view of the MTM target capsule $\ldots \ldots \ldots \ldots \ldots \ldots \ldots \ldots \ldots \ldots \ldots$

3.4 Side view of the MTM target capsule $\ldots \ldots \ldots \ldots \ldots \ldots \ldots \ldots \ldots \ldots$

3.5 Front view of the MTM capsule-transport carriage $\ldots \ldots \ldots \ldots \ldots \ldots \ldots \ldots$

3.6 Side view of the MTM capsule-transport carriage $\ldots \ldots \ldots \ldots \ldots \ldots \ldots \ldots \ldots$

3.7 Side view of the MTM carriage-capsule assembly in position at the lower end

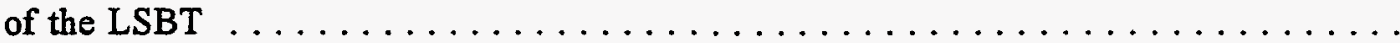

3.8 End view of the MTM carriage-capsule assembly in position at the lower end

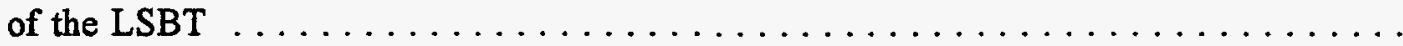

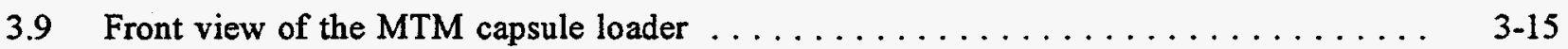

3.10 Side view of the MTM capsule loader $\ldots \ldots \ldots \ldots \ldots \ldots \ldots \ldots \ldots \ldots$

3.11 Side view of the MTM carriage-capsule loader assembly in position at the

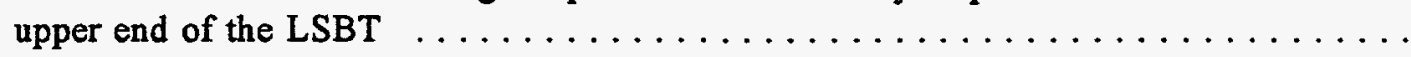

3.12 Front view of the MTM carriage-capsule loader assembly in position at the upper end of the LSBT

3.13 Rear view of the MTM carriage-capsule loader assembly in position at the

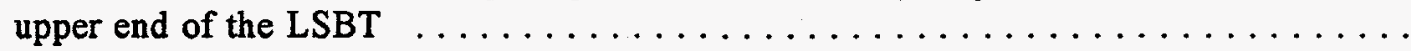

3.14 A schematic diagram of the helium-jet transport system $\ldots \ldots \ldots \ldots \ldots \ldots$

3.15 Source arrangement for the in-pile breeder ion source $\ldots \ldots \ldots \ldots \ldots \ldots \ldots$

3.16 A schematic diagram of the LSBT with the elements for use with the in-pile breeder ion source shown in place

3.17 A schematic layout of a Sidonie-type separator in the second-floor experimental area 


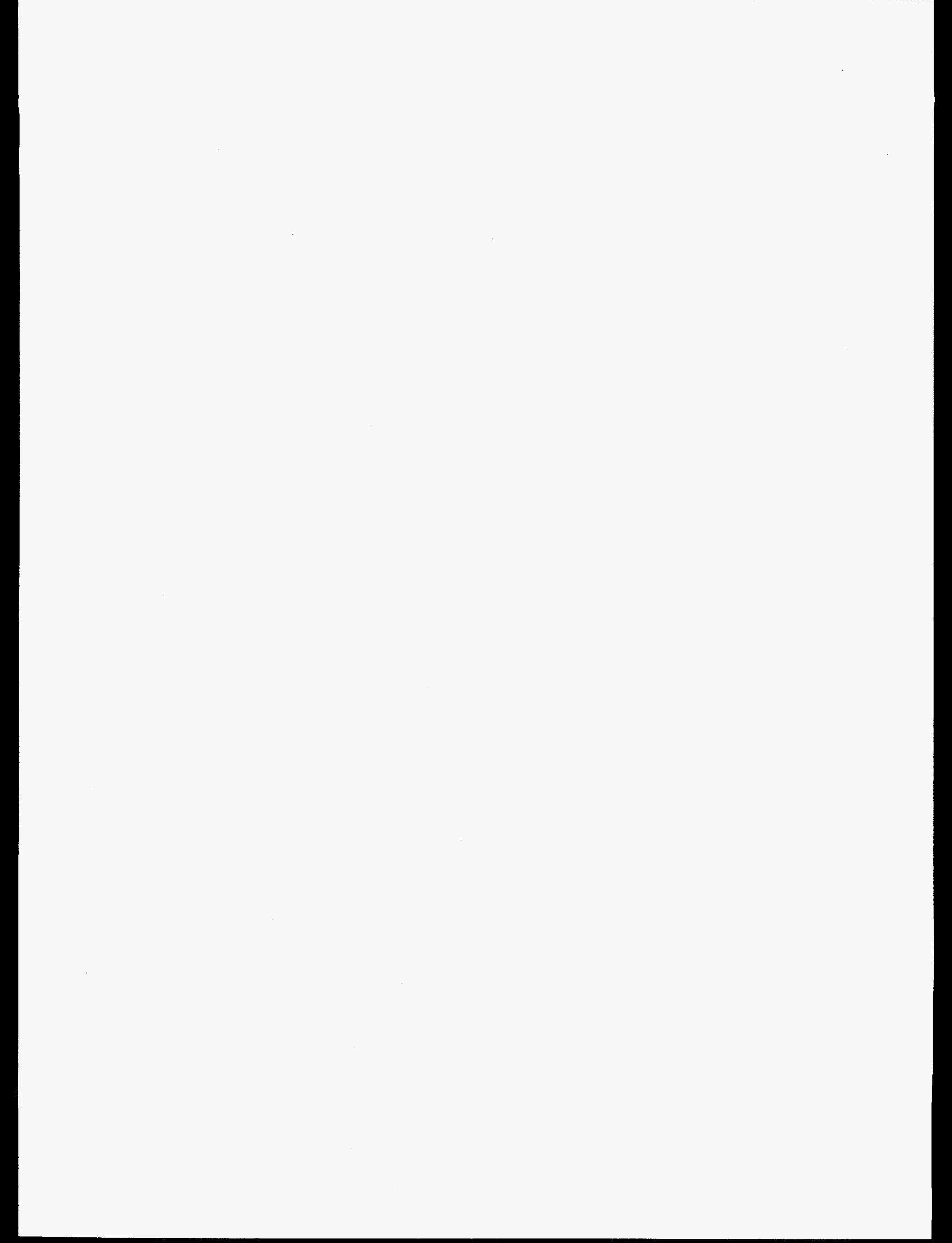




\section{ACRONYMS}

ANS

CERN

FEBIAD

FWHM

FWTM

ILL

ISOL

LSBT

LINAC

PIAFE

NIST

MTM

RIB

SDD
Advanced Neutron Source

Centre d'Etudes et Recherche Nucleaire

forced electron beam induced arc discharge

full width at half maximum

full width at tenth maximum

Institut Laue-Langevin

isotope separation on-line

long slant beam tube

linear accelerator

Production, Ionisation, Accélération de Faisceaux Exotiques

National Institute of Standards and Technology

material transport mechanism

radioactive ion beam

system design description 



\section{PREFACE}

This report summarizes work carried out during the period 1991-1995 in connection with the refinement of the concepts and detailed designs for nuclear and fundamental physics research instrumentation at the proposed Advanced Neutron Source (ANS) at Oak Ridge National Laboratory (ORNL).

Initially, emphasis was placed on refining the existing System Design Document (SDD-43) to detail more accurately the needs and interfaces of the instruments that are identified in the document. The conceptual designs of these instruments were also refined to reflect current thinking in the field of nuclear and fundamental physics. In particular, the on-line isotope separator (ISOL) facility design was reconsidered in the light of the development of interest in radioactive ion beams within the nuclear physics community.

The second stage of this work was to define those instrument parameters that would interface directly with the reactor systems so that these parameters could be considered in the basic reactor design. To this end, several different options were considered for the ISOL facility and particularly for its associated ion source. Since two of these options involved ion sources internal to the long slant beam tube, these were studied in detail. In addition, preliminary work was done to identify the needs for the target holder and changing facility to be located in the tangential through-tube.

Because many of the planned nuclear and fundamental physics instruments have similar needs in terms of detection apparatus, some progress was also made in defining the parameters for these detectors.

The work covered in this report was primarily carried out by S. J. Robinson (Tennessee Technological University), J. G. Arterburn (ANS Project group), and S. Raman (ORNL). J. Piotrowski (Swierk) and H. Faust (Institut Laue-Langevin) were involved in developing the various ISOL conceptual designs during short-term visits in 1992 and 1994, respectively. In addition, T. J. McManamy and F. J. Peretz (both from ANS) were consulted whenever interfacing with reactor structures or systems was involved. 


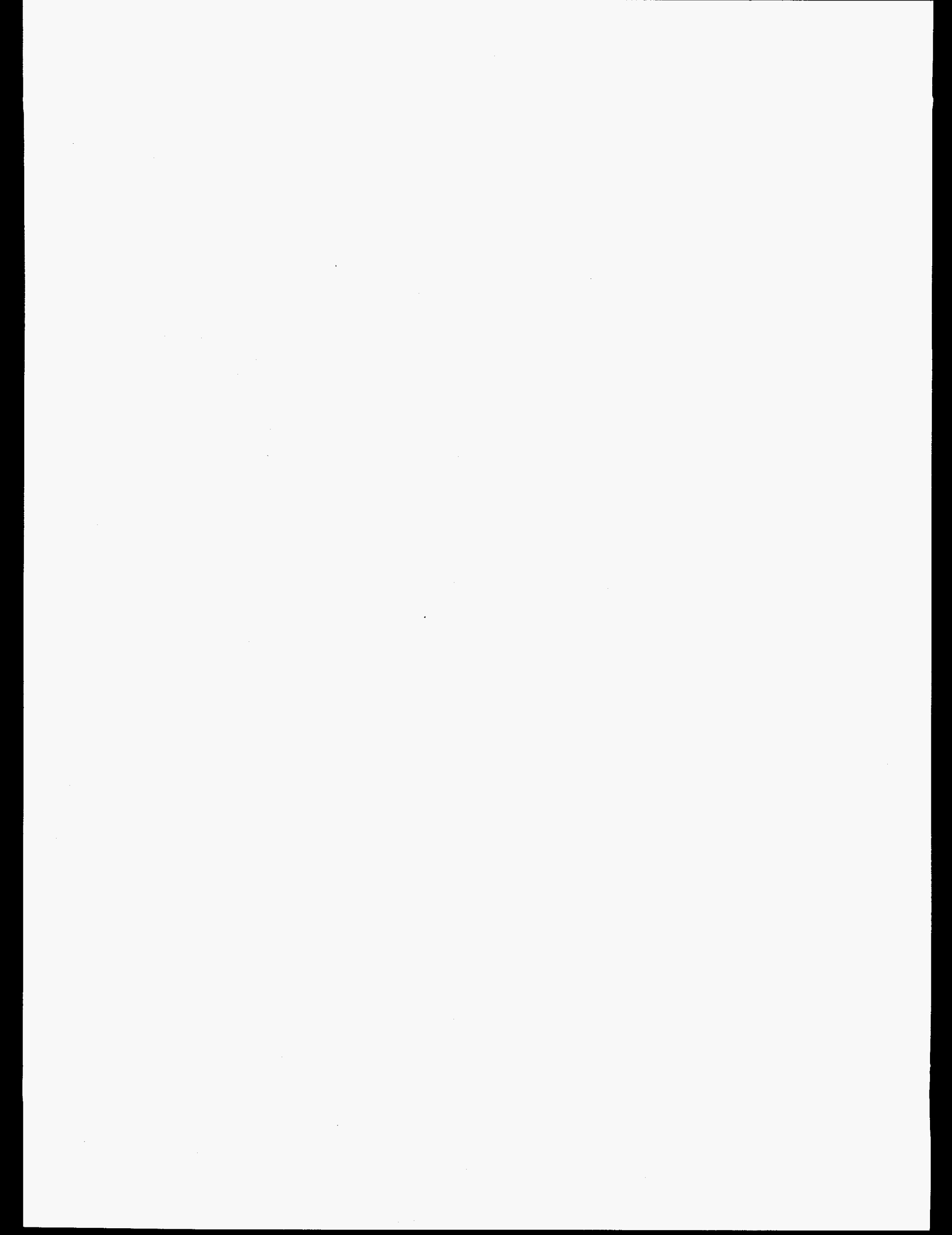




\section{INTRODUCTION}

The design parameters for the proposed Advanced Neutron Source (ANS) predict accessible neutron fluxes that are up to an order of magnitude larger than those currently available at the Institut Laue-Langevin (ILL) in Grenoble, France. The ILL is generally recognized as the world leader in the field of reactor-based neutron physics.

This increase in flux would make possible many new studies in the realms of nuclear and fundamental physics. At the most basic level, this would mean that, even with current technology, it would be possible to study processes with much lower cross sections. However, in certain cases, by taking the opportunity to interact in the design stage of the ANS reactor itself, new and exciting developments became possible. This is the case for the planned fission product isotope separation online (ISOL) facility, which could be used to form the basis of a unique neutron-rich radioactive ion-beam (RIB) installation.

The nuclear and fundamental physics instruments at ANS are classified into four categories:

- Thermal nuclear physics. With the use of a tangential through-tube, samples are introduced into the high-flux region of the reflector vessel. Gamma rays, following neutron capture, are studied using ultrahigh resolution to determine nuclear level schemes and the lifetimes of excited nuclear states.

- Polarized nuclear physics. This category includes an experimental station for the use of polarized neutron beams in weak interaction studies. Also included is an experimental station dedicated to the use of large quantities of liquid hydrogen. Finally, a low-temperature nuclear orientation facility will provide nuclear structure information and also serve as a development station for the use of a bolometer detector in precision gamma-ray spectroscopy.

- General nuclear physics. The major instrument in this category is an ISOL for the production and separation of fission products following thermal neutron capture. It would also form the basis for a RIB facility. Also included are two general purpose experimental stations to be used in a variety of nuclear and fundamental physics applications. Instrumentation would be drawn from a general pool of detectors and electronics held at ANS or would be provided by external users.

- Neutron interferometer. This dedicated instrument uses neutron interference patterns to study the fundamental interactions of the neutron itself and to determine neutron-scattering parameters, which are of importance in general neutron-scattering work.

All of these instruments will be situated in the ANS reactor and guide hall buildings. The instruments can be classified essentially as either "roll-in, roll-out" or dedicated installations. The latter may require specific interfaces to building structures or services.

When finalized, all conceptual design details and specific parameters of these instruments will be included in a system design description (SDD) for the ANS Project. The specific section dealing with nuclear and fundamental physics instrumentation is designated SDD-43. As design work has progressed, it has been incorporated into SDD-43, where appropriate. 



\section{DEFINITION OF INSTRUMENT SYSTEMS}

\subsection{REVISION OF SDD-43}

A draft baseline version of Sect. 1 of SDD-43," "Functions and Design Requirements," was released in May 1991 for comments on format and top-level requirements. The first tasks undertaken by this group were to refine SDD-43 to include more details of instrument function (Sect. 1.1) and to expand considerably the section on design requirements (Sect. 1.2) to reflect more accurately the anticipated interfaces with building structures and systems.

The most important aspect of this work was to redefine the function of the ISOL facility to include its possible use as a front-end facility for the generation of RIBs. The major impact of this change would be to provide for the passage of a low-energy ion beam from the ISOL, through the wall of the reactor containment building, to an external accelerator facility. Also detailed were the requirements for vibration isolation for several of the proposed instruments and the safety requirements for the handling of liquid hydrogen at a special station dedicated for this purpose.

After this work, a draft of Sect. 2 of SDD-43, "Design Description," was prepared. This section contains detailed descriptions of the individual instruments and experimental stations proposed for nuclear and fundamental physics at ANS. Included are the principles of operation of each instrument (where appropriate), detailed system descriptions (Sect. 2.2), system performance parameters (Sect. 2.3), system arrangements (Sect. 2.4), detailed descriptions of individual component designs (Sect. 2.5), and instrumentation and control (Sect. 2.6). A completed SDD-43 was approved, issued in June 1992, and revised in June $1993 .^{2}$ A copy of this version of SDD-43 is included as Appendix A to this report. Following the update of SDD-43, the next task of the group was to identify and attempt to complete those instruments and design specifications that would have major direct interfaces with reactor systems and structures. These were the ISOL facility and the through-tube target-loading facility. Work on these facilities is described in Sects. 3 and 4 of this report.

\subsection{PRELIMINARY BUDGETS FOR INSTRUMENT CONSTRUCTION}

Preliminary estimates were made for the budgets needed to construct the proposed instruments. Also included were estimates of the personnel needed for both the design and the construction phases. These estimates were based, where appropriate, on those of similar facilities installed elsewhere (suitably scaled), manufacturers' published price lists, and discussions with experts in the field. The budget estimates are given in Appendix B. 



\section{DESIGN STUDIES FOR THE ON-LINE ISOTOPE SEPARATION FACILITY}

\subsection{INTRODUCTION}

\subsubsection{Concept and Design Options}

The 1993 version of SDD-43 (see Appendix A) includes a preliminary design for the ISOL facility based on the Lohengrin separator at ILL. It was soon concluded that such a device would not be the most scientifically desirable for ANS, and a major effort was made to specify a more suitable design.

The ISOL facility uses the neutron flux in the large slant beam tube (LSBT) at the ANS (see Fig. 3.1) to induce fission in fissile material. The fission products are extracted and ionized before they pass into a separator stage, which is configured to allow the passage of only one particular mass chain. The separated fission products are then delivered to a measurement station for study.

The different stages of the separator assembly can be considered as being somewhat independent. Of major import to reactor systems is the ion-source assembly, for which three possibilities were identified. These are

- to make use of the neutrons exiting from the LSBT to interact with an external target and ion source at the top end of the LSBT,

- to introduce a small sample cell into the higher neutron fluxes available at the lower end of the LSBT and to extract fission fragments using a helium jet (these would be introduced into an external device for subsequent ionization), and

- to install a self-contained, in-pile breeder ion source with electrostatic extraction of ionized fission fragments and direct injection into a separator.

The first two approaches were developed in collaboration with A. E. Piotrowski of the Soltan Institute for Nuclear Studies in Swierk, Poland. They can be considered as complementary because both would be possible with a properly configured rotary shutter mechanism at the top of the LSBT (Fig. 3.1).

The third, and most radical, design (the in-pile breeder ion source) was developed in collaboration with $\mathrm{H}$. Faust of the ILL to be a dedicated facility, which was foreseen to remain in place for the life of the LSBT.

Before the development of the in-pile breeder ion-source approach, a considerable amount of work was done on detailing the mechanism for loading and unloading a remote target cell into the LSBT. This mechanism was dubbed the material transport mechanism (MTM) and is described in detail in Sect. 3.3.1.

Two draft internal reports were prepared concerning the individual stages of the separator assembly and considering the possible options for each stage, based on present and predicted future capabilities and already existing and planned facilities. This section of the current report attempts to draw together the work accomplished on possible designs for the ISOL facility and to make recommendations for any future developments.

\subsubsection{Production of Radioactive Ion Beams}

The study of nuclear structure using stable, heavy-ion beams is an established practice. Recently, several facilities have been proposed to produce RIBs so that different nuclei can be studied. These facilities will concentrate on proton-rich RIBs, which allow the study of nuclei closer to the proton 


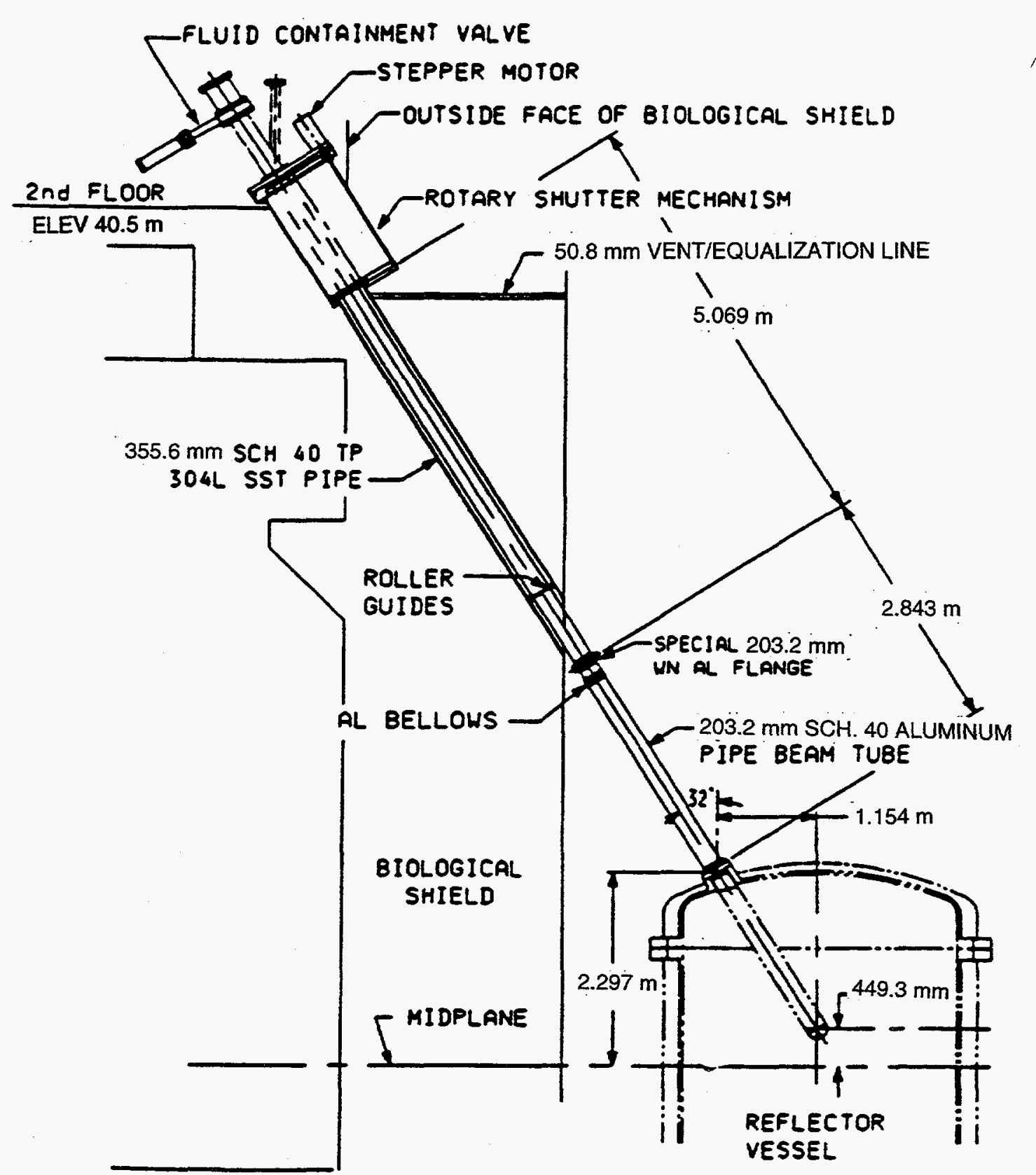

Fig. 3.1. The LSBT at the ANS. The helium-jet target capsule would be lowered to a point just outside the reactor reflector vessel. The ion sources and separator would be located at the upper end of the LSBT, which is in the second-floor experimental area. 
drip line. However, adding neutrons to a stable configuration may also considerably change the behavior of nuclear forces and strongly modify the spectroscopic properties near the neutron drip line. It is therefore important to choose nuclear reactions that allow the production of exotic nuclei with neutron excesses of about 15 to 20 neutrons above the last stable configuration.

The most suitable reaction to produce these exotic nuclei is thought to be thermal neutron-induced fission, ${ }^{3 *}$ following which primary reaction products with large neutron excesses may be extracted. The maximum yield for fission products occurs for isotopes having neutron excesses of about 8 to 9 neutrons above the last stable configuration. For an intense fission source, it is possible to extract exotic species having neutron excesses as far as 15 to 20 neutrons above the last stable configuration. For many isotopic chains, this would represent nuclei very close to the neutron drip line.

The first stage in generating a heavy-ion beam is to produce a stream of charged ions for injection into an accelerator, such as a linear accelerator (LINAC) or a Van de Graaf machine. At the ILL, the Production, Ionisation, Accélération de Faisceaux Exotiques (PIAFE) project is already in progress. ${ }^{4,5}$ This project makes use of an in-pile ion source in the high-flux reactor to produce exotic isotopes and to accelerate them further to energies above the Coulomb barrier. The project will be installed at the ILL reactor using an existing standard beam tube. This approach has definite advantages in terms of budget requirements and time scale for the realization of the project. On the other hand, the existing infrastructure leads to a considerable loss of freedom in the concept and the design of the ion source. Furthermore, a dedicated beam tube is not available. The preferred beam tube configuration is an inclined tube with a large diameter.

The ANS separator could be used also to produce such a beam (from the isotopes separated after neutron-induced fission) for injection into an external accelerator facility. The concept of the ANS makes it possible to define the optimum conditions for the implementation of an exotic beam facility beforehand. Furthermore, the increased neutron flux opens new possibilities not available elsewhere.

Another major consideration in the ISOL design was the provision for a future passage of the mass-separated ion beam through the reactor building wall. This would allow the separator to be used as a source of neutron-rich isotopes for the production of neutron-rich RIBs using a heavy-ion accelerator external to the reactor building itself.

\subsection{ION SOURCES-DETAILED DESIGN OPTIONS}

\subsubsection{Combined Target/Ion Sources ${ }^{1}$}

The most conventional approach is to allow neutrons to traverse the LSBT and emerge through the shutter to interact with fissile material contained in an external ion-source assembly. (The rotating shutter mechanism will have holes of different diameter to allow for collimation of the neutron beam reaching the target.) Several different types of ion source are possible, depending on the particular application; the most important of these are discussed in Appendix C. All involve the basic principle of first thermalizing the recoiling fission products and then ionizing and delivering them to the separator stage.

\subsubsection{General Features}

The target should have a large surface area to enable all fission products created in the bulk of the target material to reach a surface quickly. The diffusion of the thermalized fission products in the

\footnotetext{
*H, Nifnecker, ed., Project Physics Case, 1993 (unpublished).
} 
target matrix depends on the mobility of the particular element involved, the material of the target matrix (usually graphite), and the temperature. A high temperature is needed to encourage quick desorption from the surface into the ionization region. Such conventional ion sources at reactors have been placed either at in-pile positions (e.g., at the University of Mainz, Germany and at the Studsvik Neutron Research Laboratory, Sweden) with neutron fluxes on the order of $1 \times 10^{15} \mathrm{~m}^{-2} \cdot \mathrm{s}^{-1}$ or as external facilities outside the reactor vessel containment (Brookhaven National Laboratory) and on neutron guides (ILL, Grenoble) at neutron fluxes below $1 \times 10^{13} \mathrm{~m}^{-2} \cdot \mathrm{s}^{-1}$. The total mass of ${ }^{235} \mathrm{U}$ used in these sources was a few grams, for which the fission rate is far from sufficient to heat the devices up to $-2400^{\circ} \mathrm{C}$, the temperature required for the fast release of short-lived fission products to be investigated. As a consequence, external heating is required for these sources, and additional heat shields and cooling are necessary for proper operation.

After leaving the target matrix, the fission products are ionized by whatever mechanism the source was designed to use, and then, because the ionization region is at a higher potential than its surroundings, the ions can be extracted from any hole in the ionization enclosure. Therefore, it is extremely important for the ion source to be hermetically sealed with only one escape pathway for the ions. The ion beam is formed using an extraction electrode placed a few millimeters outside the ion-source exit orifice. The ions are accelerated across the high potential difference (typically around $40 \mathrm{kV}$ ) between the ion source and the extraction electrode.

The consequence of these requirements is a rather involved design for the source, leading to many components subject to failure. Usually, the lifetimes of such sources are on the order of $2000 \mathrm{~h}$, determined mostly by failures of the filament heating system, vacuum components (O-rings and water seals), and electrical insulators.

Another consideration is that, because certain parts of these ion sources are likely to become highly active during their lifetimes, they should incorporate easily interchangeable elements and that time periods between these changes should be as long as possible. Thus, the need is for highly flexible, reliable, and long-lived ion sources. When ion-source elements (or the sources themselves) must be changed, provisions must be made for the handling and disposing of the active elements.

Use of such an ion source at the ANS assumes that its installation is at the upper end of the LSBT. Neutron fluxes at this position are estimated to be of the order $5 \times 10^{13} \mathrm{~m}^{-2} \cdot \mathrm{s}^{-1}$. Assuming the target material to be $-5 \mathrm{~g}$ of ${ }^{235} \mathrm{U}$, this would lead to a fission rate of $3.6 \times 10^{10} \mathrm{fissions} / \mathrm{s}$. (This translates to a total inventory of fission products of about $6 \mathrm{Ci}$, all contained in the experimental hall.) This fission rate is not sufficient to ensure heating of the source to the required $2400^{\circ} \mathrm{C}$, and so the usual external heating, water cooling, and heat shields would be required. All of these complications mean also an expected source lifetime of about $2000 \mathrm{~h}$. However, it should be noted that the position of the ion source at the upper end of the LSBT means that no major interface with reactor systems is necessary to effect regular repairs and maintenance.

However, the main disadvantage to this approach is that it is very unlikely that any new physics would emerge from it because this type of fission source has been extensively used for the last 20 years. In particular, access to very exotic isotopes far from stability seems to be almost excluded. The yield of these isotopes is about $10^{-9}$ of the fission yield, which, with the previously discussed fission rate, is too low to be detected if losses resulting from extraction are considered.

\subsubsection{Mode of Operation}

When the separator is to be operated in the combined target/ion-source mode, the MTM will not be used. In this case the empty transport carriage will be raised to the top of the ball screw and left there. An appropriate opening in the rotary shutter mechanism will be selected to collimate the neutrons exiting the LSBT and interacting with the external target. 


\subsection{INTERNAL TARGET AND HELIUM-JET ION SOURCE}

The basic idea of a helium-jet ion source is to have a target of fissile material in a remote cell in a high neutron flux. This cell is flushed continuously with helium gas, which is saturated with aerosol clusters of a carrier material. These aerosol clusters carry the fission products through a capillary tube several meters in length to the ion source, where the fission products are removed, ionized, and injected into the separator stage.

The main advantage of such a system is that the sometimes considerable delays associated with diffusion through the target matrix and desorption at the target surface do not occur, even for high-melting-point elements. Thus, many elements not available with more conventional ion sources can be studied. The primary limitation is the ability of the system to handle the high helium gas load, which is an inevitable consequence of fast and efficient transport of the fission products.

\subsubsection{Material Transport Mechanism}

\subsubsection{Design Requirements}

In order to achieve the objectives for nuclear physics experiments, the following design requirements for the MTM must be met.

- The target material must be sealed in a capsule with access ports for capillary tube connection.

- A capillary tube system must supply helium gas to the capsule and return with fission products to an experimental area.

- The capsule must be positioned in the LSBT at a point of acceptable neutron flux.

- The capsule must be retrievable from the LSBT.

To satisfy these requirements, a system has been designed in which the target cell will be mounted on a carriage mechanism for transport up and down the LSBT (Fig. 3.2). This carriage will operate on a ball screw. Insertion and removal of the target cell from the LSBT will be via a separate capsuleloading mechanism, which will pass through an opening in the rotary shutter. Connection to the fixed helium-transport helium-capillary tubes will be made at the bottom of the LSBT, via automatic connectors.

\subsubsection{Target Cell}

In order to make use of the high neutron flux at the lower end of the LSBT, a target cell containing foils impregnated with fissile material will be introduced into the LSBT to a position just above the point at which the LSBT interfaces with the reactor reflector vessel. The dimensions of the target cell are limited by the dimensions of the LSBT ( $200 \mathrm{~mm}$ diam) and any transport mechanism necessary for the insertion of the cell itself. The most efficient geometry would be a cylindrical chamber several inches in diameter and in length into which could be placed stacks of foils containing fissile material. Figures 3.3 and 3.4 show a possible alternative geometry, based on a square cross section of $9 \mathrm{~cm}$ on each side. This geometry offers ease of construction and handling but may be less efficient than other geometries in terms of helium flow. The target capsule will be aluminum. Both ends of the target capsule will accommodate thin windows of a low-activation material, such as beryllium, to allow the passage of neutrons through the cell and to minimize the activation of the cell material itself. 


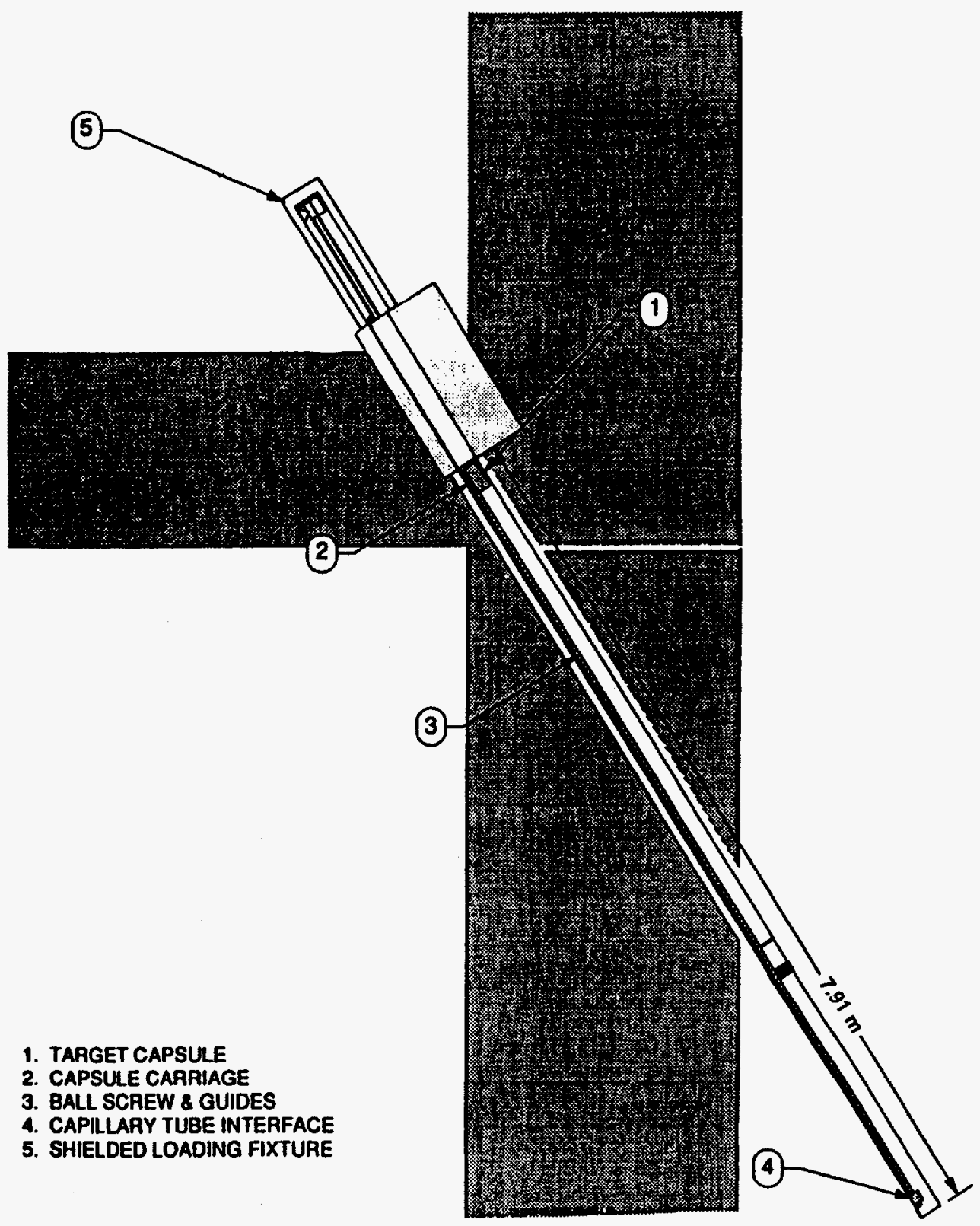

Fig. 3.2. The main elements of the MTM in relation to the LSBT. 


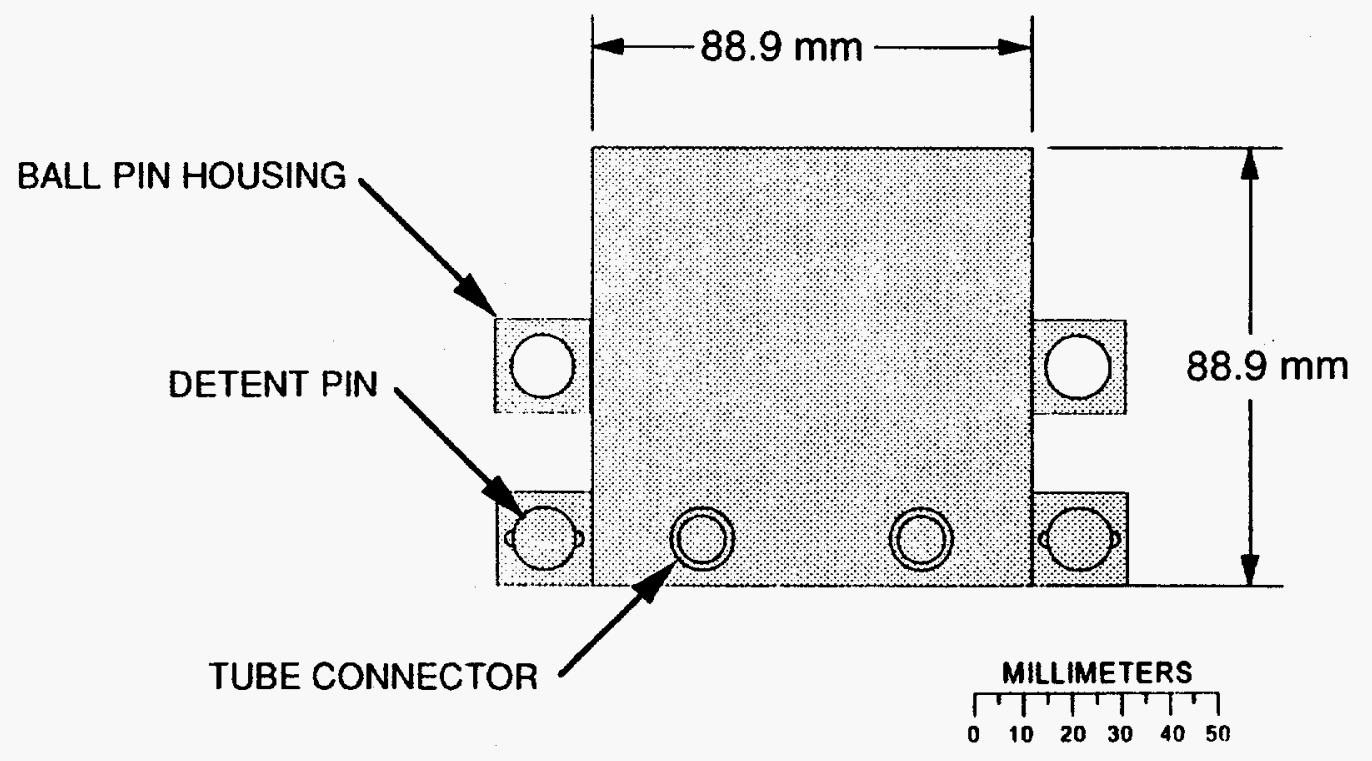

Fig. 3.3. Front view of the MTM target capsule. 


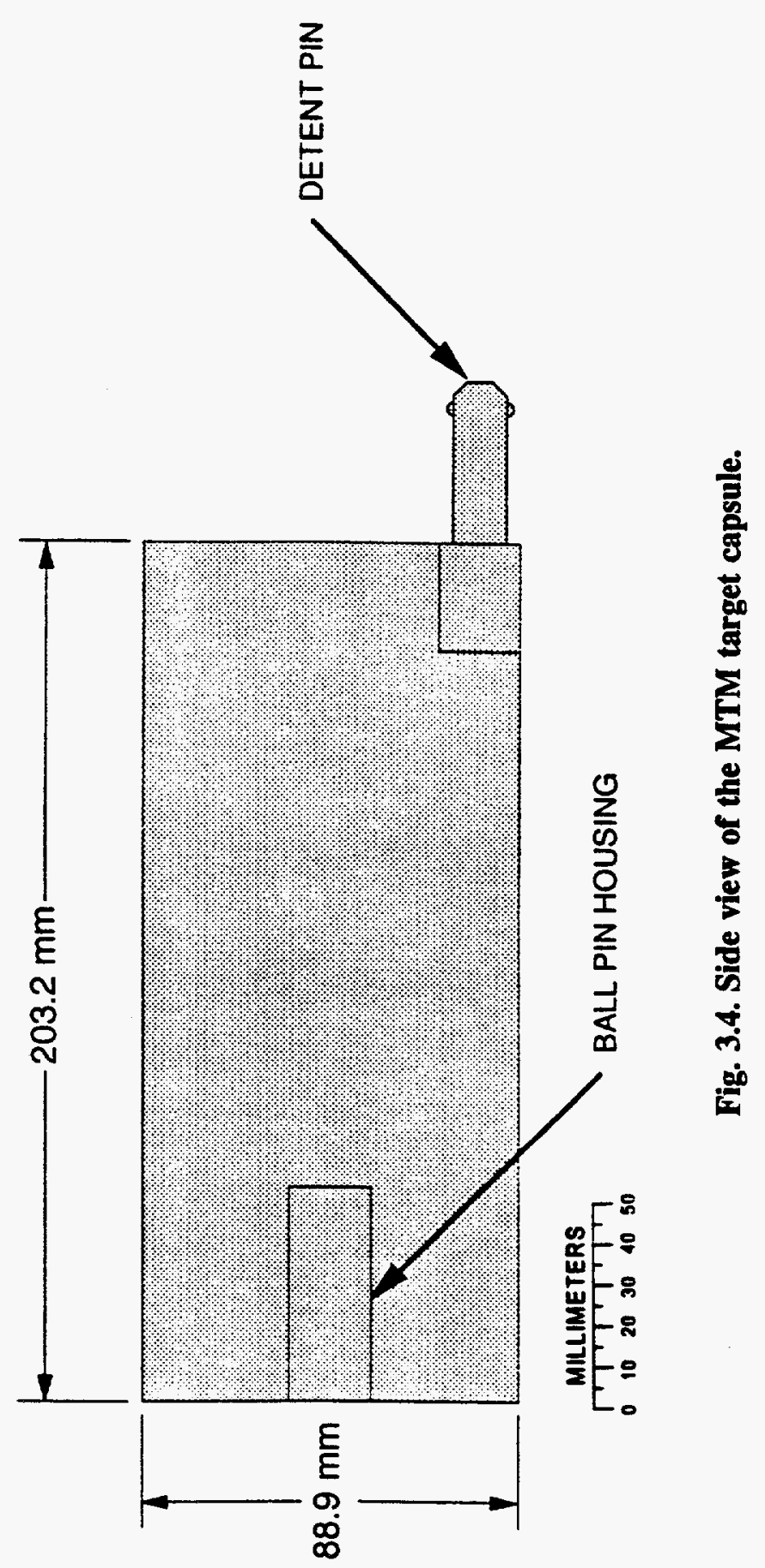


Target cell dimensions of about $200 \times 90 \times 90 \mathrm{~mm}$ will allow about $2.7 \mathrm{~g}$ of target material in the form of nickel-supported $93 \%$ metallic uranium foils of thickness $-10 \mathrm{~g} / \mathrm{m}^{2}$. These foils will allow most of the fission products to escape into the helium atmosphere. Fission products leave the target foils almost unslowed and lose their kinetic energy in the surrounding helium. The helium gas carries sodium chloride clusters to which the fission products attach. The clusters are then swept away to the ion-source assembly located in the separator enclosure in the second-floor beam room.

The increased neutron flux at this position (estimated to be about $5 \times 10^{17} \mathrm{~m}^{-2} \cdot \mathrm{s}^{-1}$ ) then leads to a fission rate of $2 \times 10^{14}$ fissions/s with a heating power of $6 \mathrm{~kW}$. The half-life of the target is $-235 \mathrm{~d}$. It is understood that the heating power must be evacuated by the helium gas itself and by radiative cooling. Preliminary calculations have shown that this heat evacuation is possible.

Many different geometries have been tested for target-cell inlet and outlet manifolds (for helium-gas circulation), but it has been found that a simple arrangement of two inlets and one outlet is an efficient design for transporting short-lived activities. ${ }^{6}$ With a target cell pressure of $500 \mathrm{kPa}$, transit times as low as $230 \mathrm{~ms}$ have been recorded for a $22-\mathrm{m}$-long capillary transport tube. Capillary lengths less than $10 \mathrm{~m}$ will be needed for the ANS system, but significant improvement in transit time is not expected because it has been determined that target chamber dwell time dominates any such transport process. ${ }^{7}$ Transport efficiencies of up to $60 \%$ have been reported for such systems.

The inlet and outlet valves (for the helium flow) will be of the automatic quick-disconnect type. These stainless steel connectors will be located on the front face of the capsule (Fig. 3.3). When the capsule is in position, these valves will mate with their counterparts at the lower end of the LSBT (see below) to complete the helium circulation system. Until a positive mating is achieved, these connectors will remain closed so that no release of target material into the volume of the LSBT is possible.

The capsule will have locking pins on its front face to hold it in position on the transport carriage and ball-pin housings on the back end to interface with locking ball pins on the capsule-loading mechanism (Figs. 3.3 and 3.4).

\subsubsection{Transport Carriage}

The target capsule will be transported up and down the LSBT on a carriage transport mechanism. The target capsule will be held on the transport carriage with two locking pins (located on the front face of the capsule), which will engage with locking-pin housings on the front of the transport carriage.

The carriage frame will be made of aluminum. Ball slides will be mounted to the frame and ride on guides fixed within the LSBT. A ball nut will be mounted on a support welded to the carriage frame. The ball nut will travel on a ball screw fixed within the LSBT. Détente pins at the front of the carriage will engage with housings fixed to the lower support of the ball screw. Locking-pin housings will provide attachment points for the capsule.

Figures 3.5 and 3.6 show front and side views of the carriage alone. Figures 3.7 and 3.8 show the combined carriage and target capsule assembly at its interface with the capillary tubes at the lower end of the LSBT.

\subsubsection{Ball Screw}

A stainless steel ball screw will be rigidly supported at bearings on both ends and will provide the drive mechanism to position the carriage along the LSBT (Fig. 3.2). The top bearing will allow penetration of the ball-screw drive shaft. The drive shaft will engage the driving motor mounted on the capsule loader assembly. The bottom bearing will be mounted to a support that also has locking-pin 


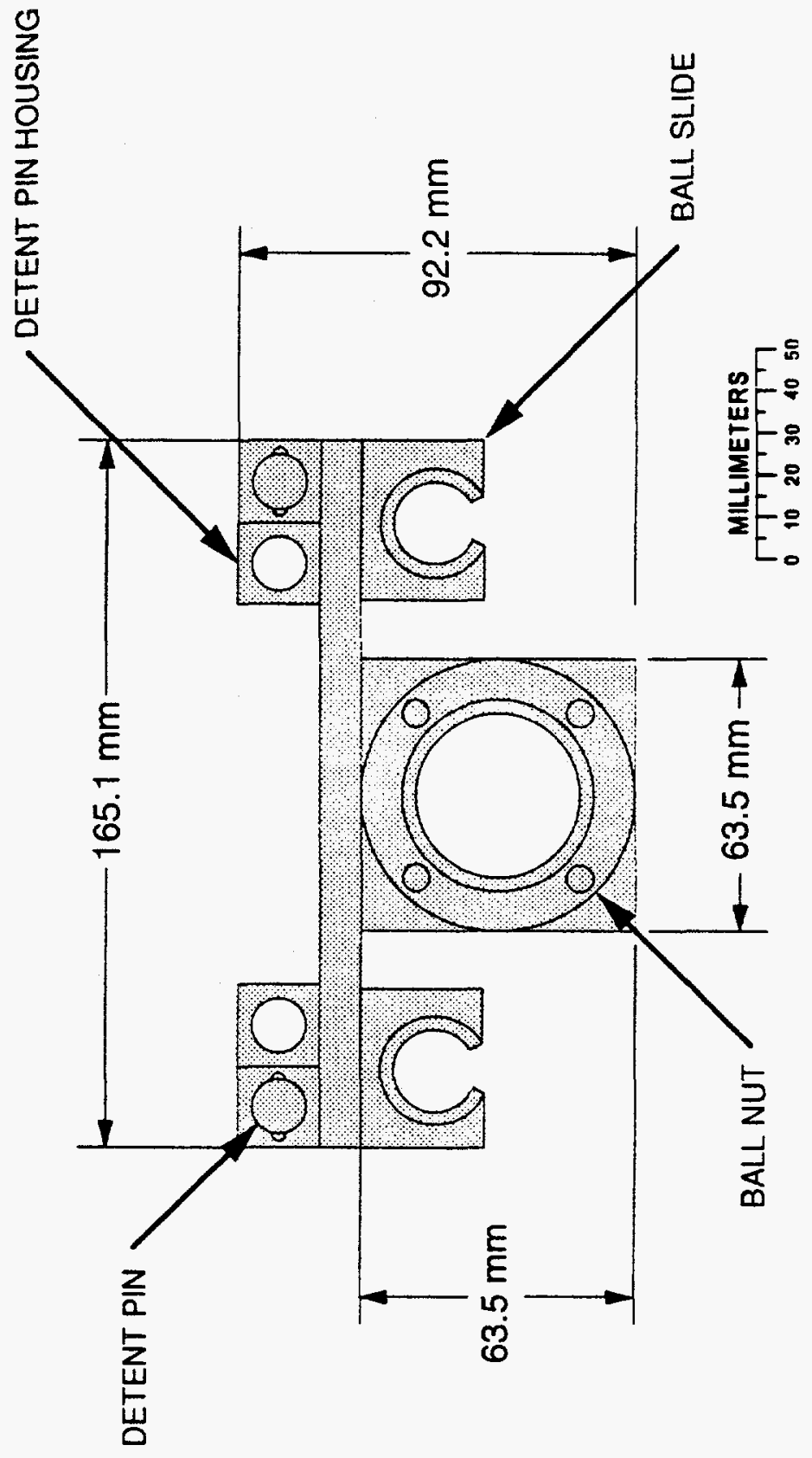




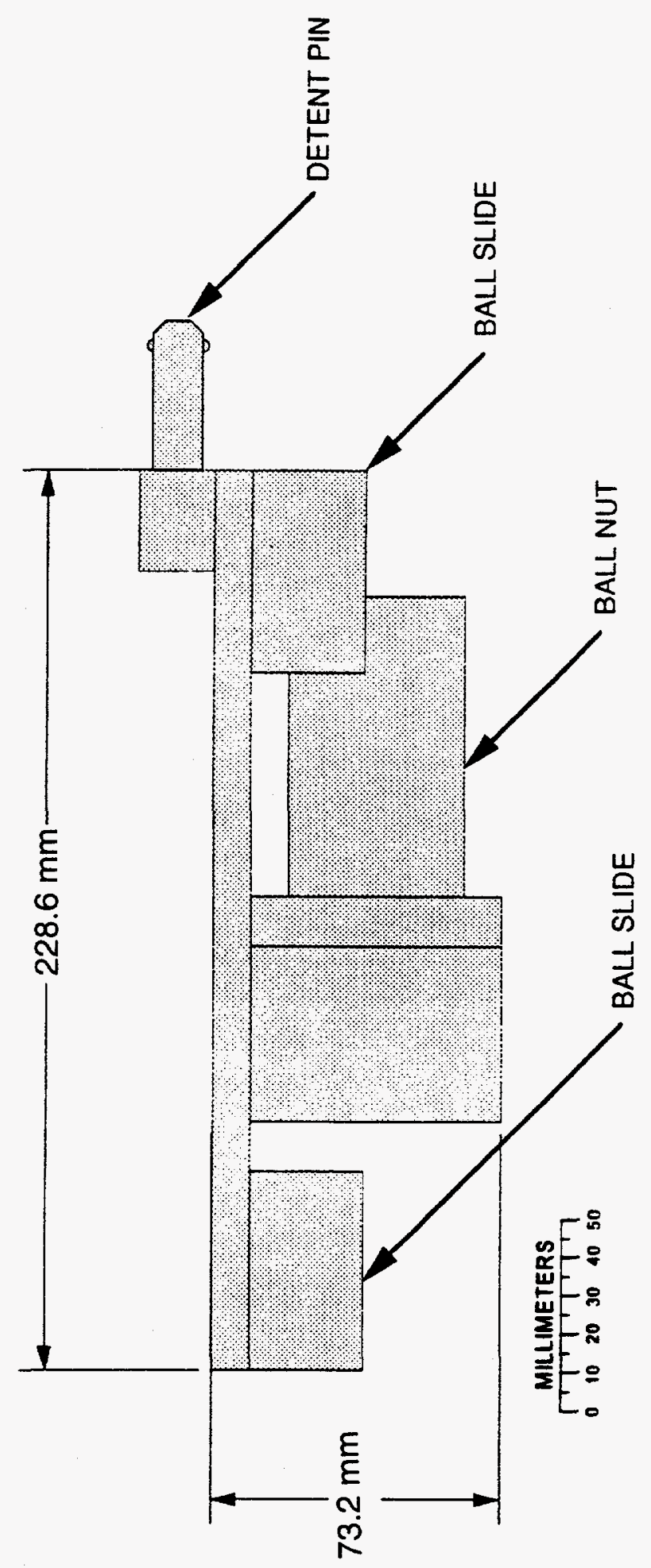




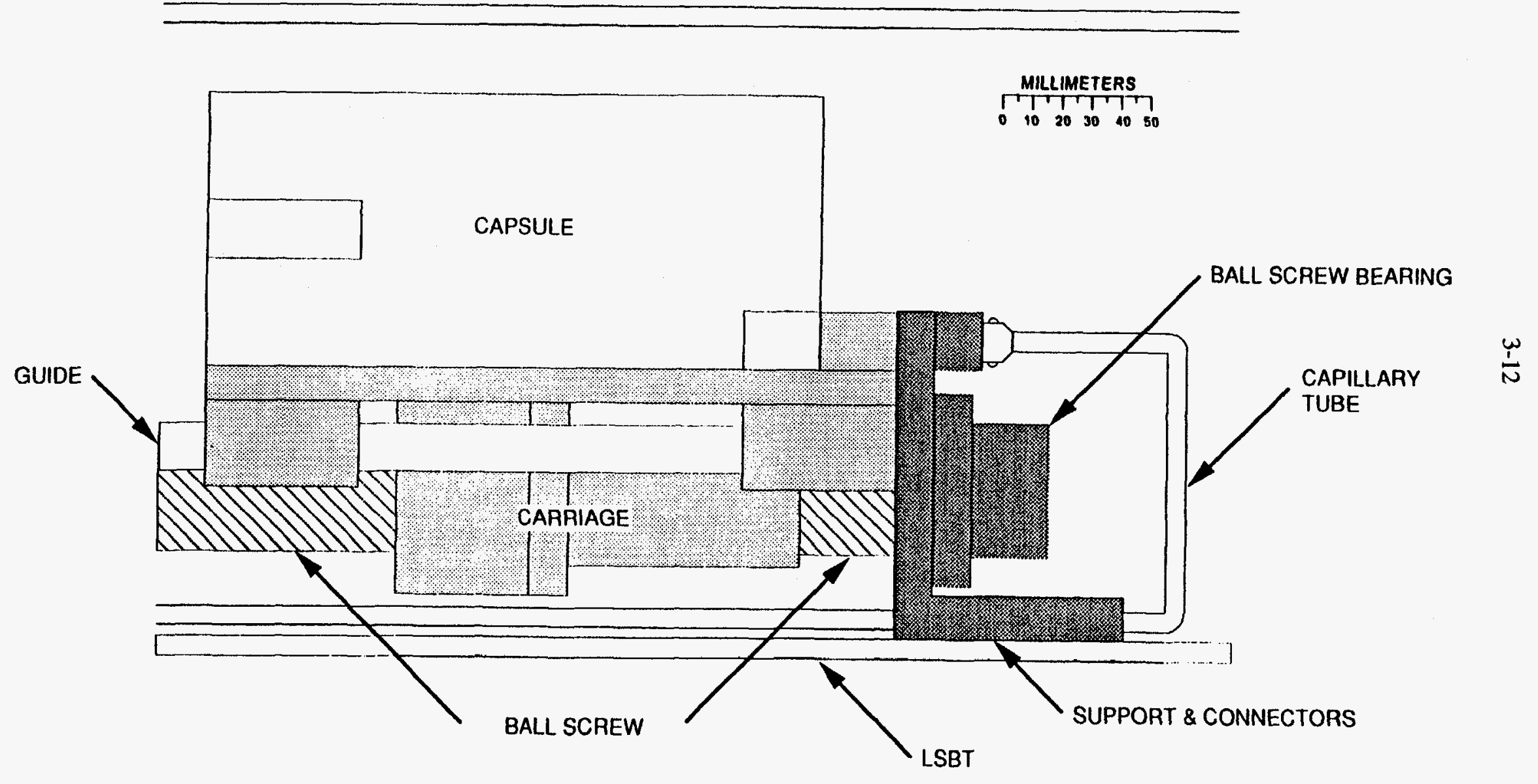

Fig. 3.7. Side view of the MTM carriage-capsule assembly in position at the lower end of the LSBT. 


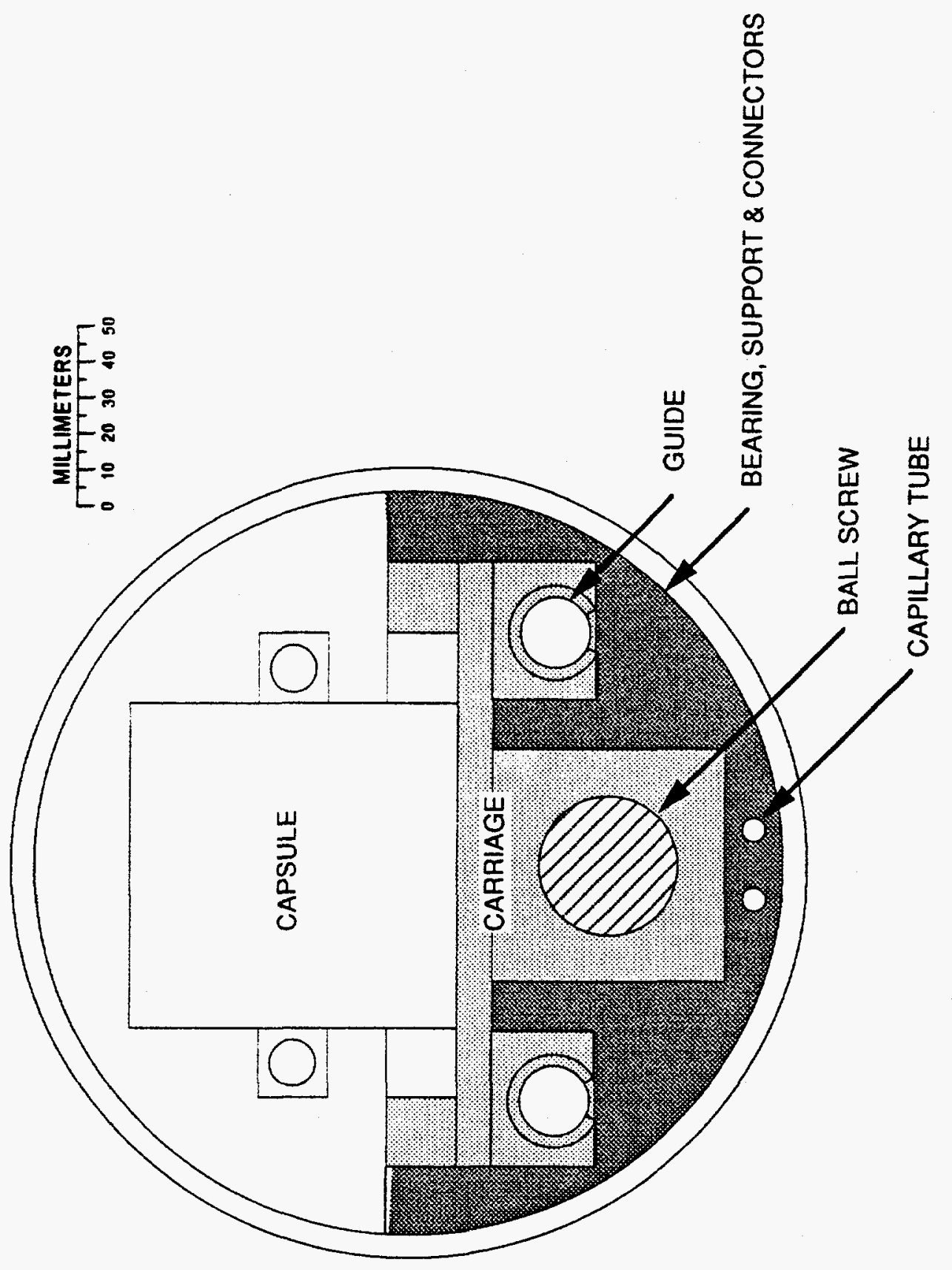

星 
housings for locking the transport carriage in place and mating couplings for connection of the helium capillary tubes to the target capsule (Fig. 3.7).

\subsubsection{Guide Rods}

Two sets of guide rods are used in conjunction with the ball slides. One set supports and guides the capsule-loader assembly (see below). The other set supports and guides the transport carriage (Fig. 3.8). The rods will be made from stainless steel and will be supported at specified intervals.

\subsubsection{Capsule-Loader Mechanism}

Loading and removal of target capsules will be through the large hole in the rotary shutter at the top of the LSBT (Fig. 3.2). The capsule-loader assembly will pass through this hole to deliver or extract a target capsule from the transport carriage while the carriage is at the upper end of the LSBT.

The loader frame will be aluminum, and ball slides will be mounted to the frame and run on guides fixed in the rotary shutter passage. Locking ball pins with actuators will secure the target capsule during its loading and will disengage the capsule from the transport carriage during its withdrawal.

A motor will be mounted underneath the loader frame. The motor shaft will be fitted with a coupling to engage and drive the transport ball screw to facilitate the movement of the transport carriage up and down the LSBT.

Figures 3.9 and 3.10 show a front and side view of the loader alone. Figures 3.11, 3.12, and 3.13 show the capsule-loader assembly at its interface with the transport carriage at the top of the LSBT.

\subsubsection{Shielded Cask}

A shielded cask will be attached to the top face of the rotary shutter mechanism during operation of the material transport mechanism (Fig. 3.2). This cask will serve

- to minimize the residual radiation escaping from the LSBT when the rotary shutter is in its fully open position,

- to shield the activated target capsule after removal from the LSBT, and

- to transport the target capsule and loader mechanism to and from the separator enclosure.

To facilitate these operations, the cask will have a closure on its lower end. The closure can be opened when the cask is attached to the rotary shutter and can be closed before the cask is removed. The cask will also have internal mountings to allow for the insertion and removal of the capsuleloader mechanism.

\subsubsection{Helium Transport System}

\subsubsection{Capillary Tubes}

The transport of helium gas to and from the target cell will be achieved using stainless steel capillary tubes of $\sim 2-\mathrm{mm}$ internal diameter. These tubes will be fixed inside the LSBT, with upward-facing coupling connectors fixed on the lower ball-screw support (Fig. 3.7). These couplings 


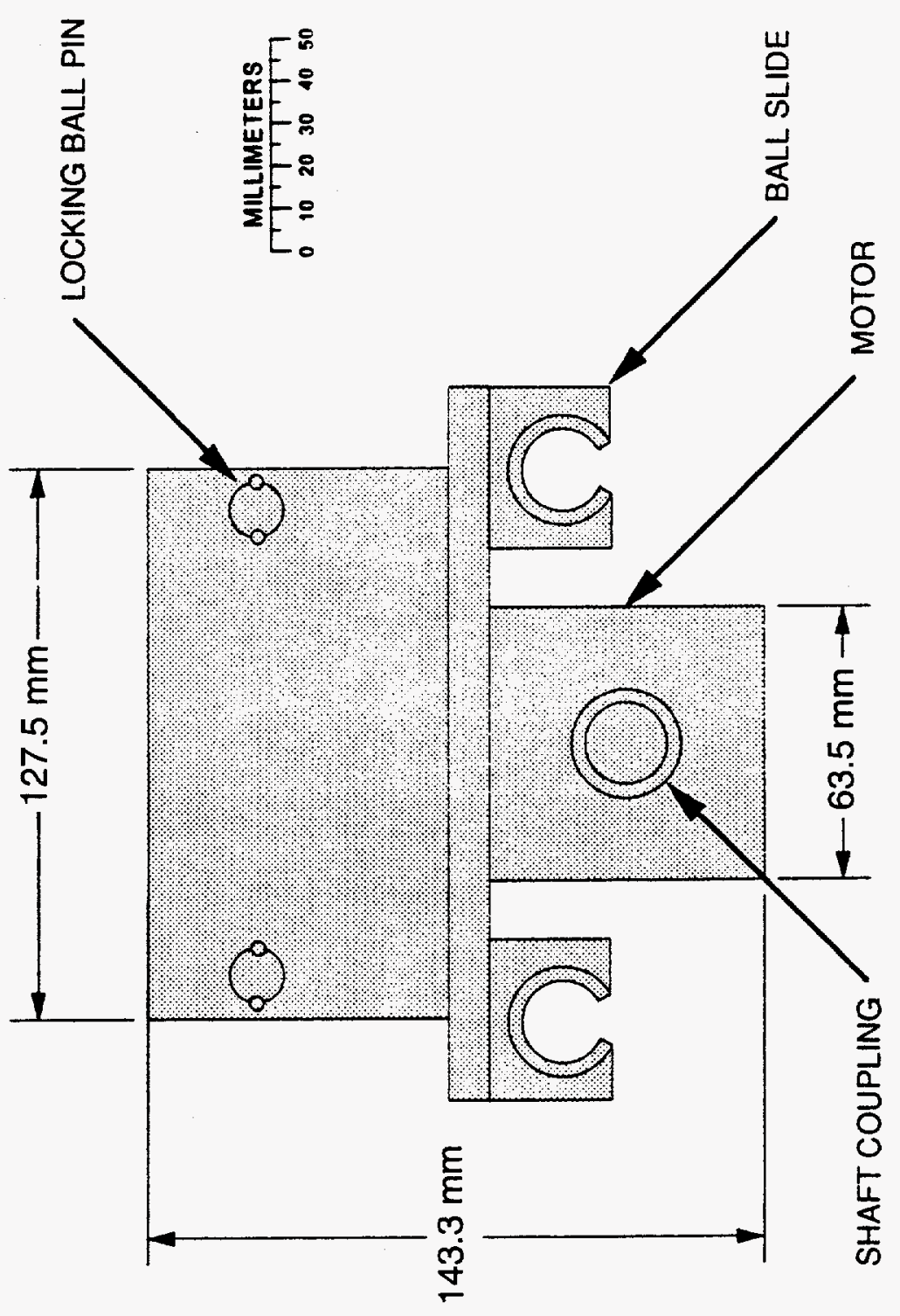

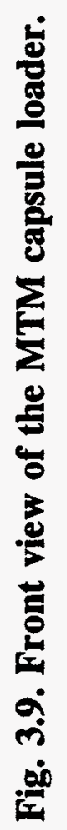




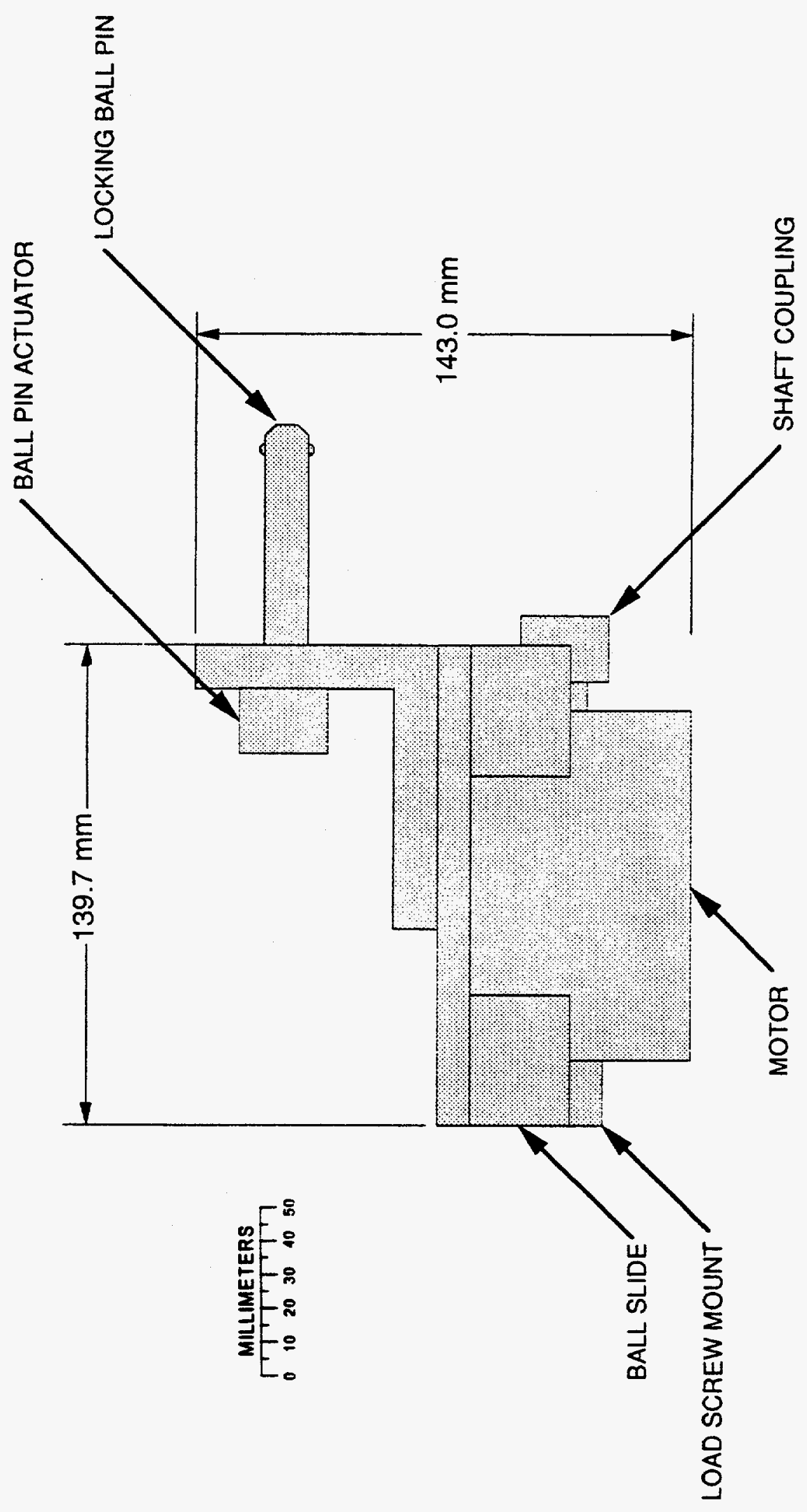

尊 


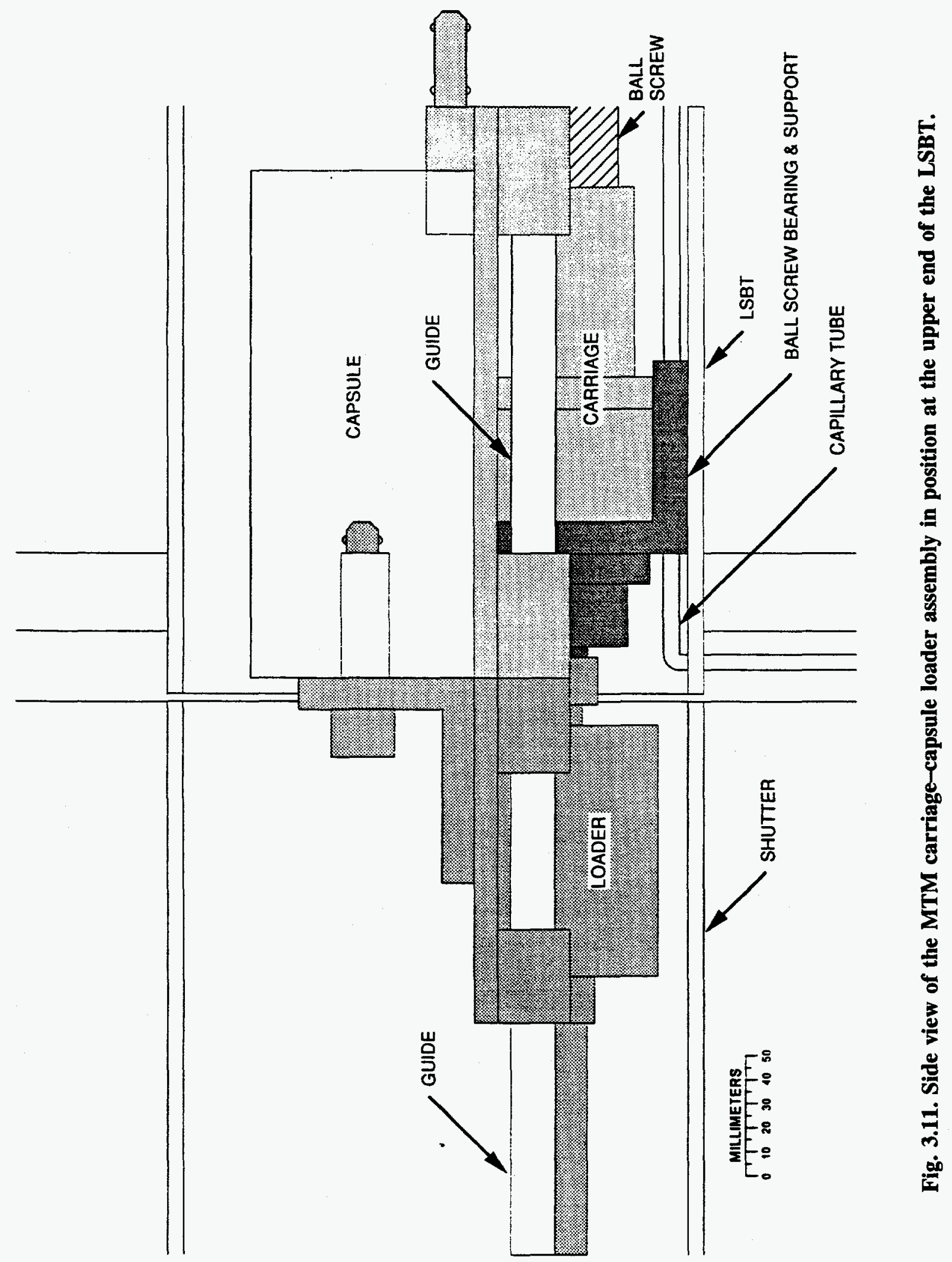




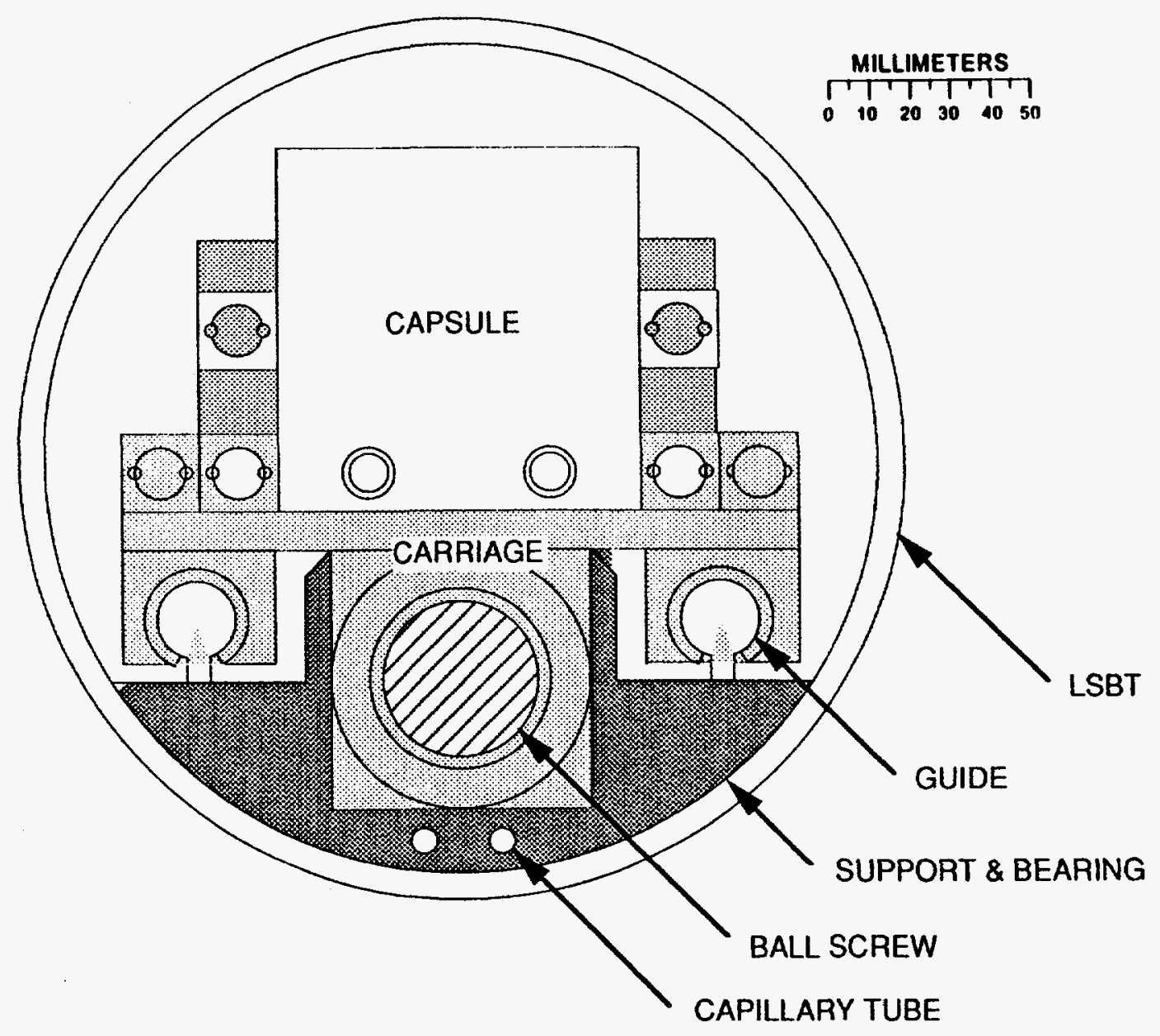

Fig. 3.12. Front view of the MTM carriage-capsule loader assembly in position at the upper end of the LSBT. 


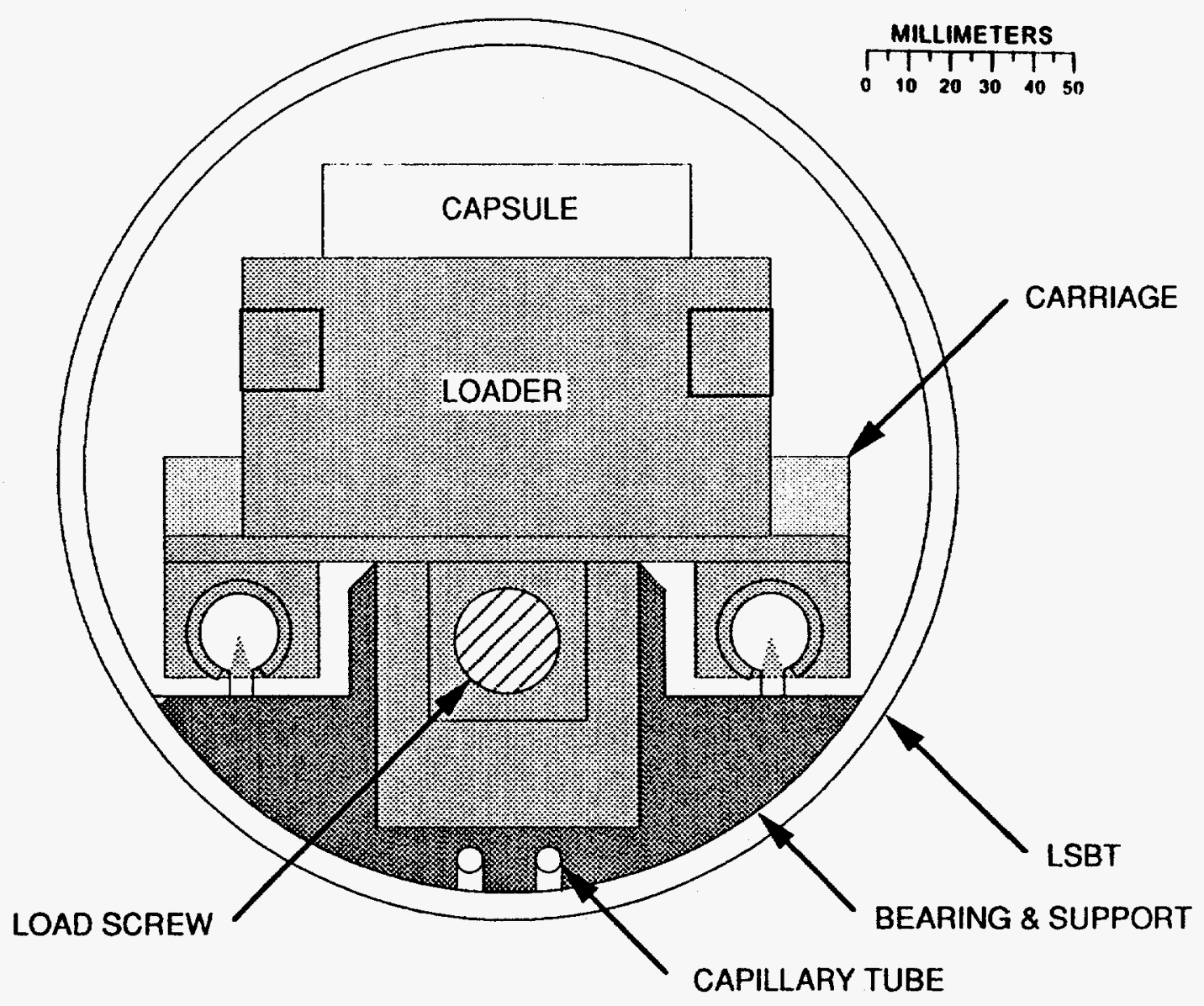

Fig. 3.13. Rear view of the MTM carriage-capsule loader assembly in position at the upper end of the LSBT. 
will engage with the mating couplings on the front face of the target cell (Fig. 3.3) and will engage when the cell is lowered into its final position. Until positive mating is achieved, these connectors will remain sealed.

The capillary tubes themselves will exit from the LSBT at a position below the rotary shutter mechanism and be fed into the separator enclosure on the second-floor experimental beam room. Provision will be made to shut off these tubes in the event of a system failure. Typical helium flow rates in the capillary tubes are 3,000 to $300,000 \mathrm{~mm}^{3} / \mathrm{s}$, and ultimately all of this gas is delivered into the ion source/separator stage. Therefore, the pumping systems of the latter must be designed to accommodate a gas load much higher than normal ISOL systems.

The normal choice for aerosol material in such systems has been $\mathrm{NaCl}$, but it has been found that $\mathrm{PbCl}_{2}$ can be more efficient. ${ }^{6}$ Aerosol particles are produced by passing the helium-gas stream over a ceramic boat containing the aerosol material heated to $-25^{\circ} \mathrm{C}$ below its melting point.

Currently existing helium-jet systems use commercial-grade helium, and no special purification measures are taken. However, the large neutron flux at the lower end of the LSBT will mean significant activation of some impurities in the helium and also of the aerosol materials. It has yet to be determined, however, whether this contamination will be significant enough to necessitate the use of high-purity gas or the search for a more suitable aerosol material. Figure 3.14 shows a schematic view of the components of a helium-jet transport system. The high efficiency of such a system is attractive. However it must be understood (1) that nearly all fission activity, including noble gases, is transported to the experimental hall and (2) that considerable shielding radioactive handling capabilities will be needed.

\subsubsection{Skimmer System}

Most of the helium conducted by the capillary is removed by a skimming process before it reaches the ion source. After its transport through the capillary, the helium jet, containing the aerosol clusters to which are attached the fission products, exits from a nozzle, which is a short distance (typically a few millimeters) from a flat skimmer plate, into which is bored a small orifice of $\sim 1 \mathrm{~mm}$ in diameter. The skimmer plate itself should be only about $1 \mathrm{~mm}$ thick in the region of the orifice, but it should be thicker farther out to accommodate cooling channels. (The alignment of the capillary nozzle relative to the skimmer orifice is crucial; consequently, provision must be made for adjusting it on an empirical basis.)

Upon streaming out of the nozzle, most of the helium gas expands into a wide cone and is pumped away by a roots pump, which has a pumping speed of $2000 \mathrm{~L} / \mathrm{s}$ or more. Most of the activity-laden aerosol particles are relatively heavy; therefore, they leave the nozzle in a narrow cone and penetrate the zone of helium turbulence (ahead of the skimmer plate) undeflected. They then pass through the skimmer orifice into the body of the ion source. It has been shown that with this simple arrangement, some $97.5 \%$ of the helium can be skimmed off, while essentially all (>90\%) of the fission products that are transmitted through the transport capillary pass through the skimmer orifice. ${ }^{8}$

If required, the amount of helium skimmed off can be increased to $99.85 \%$ through the use of a side jet that produces a curtain of helium across the entrance of the skimmer orifice. ${ }^{7}$ This side jet is most effective when its throughput is equal to that of the transport capillary and its use does not affect the transmission of the aerosol clusters through the skimmer orifice. However, it has been found that the effectiveness of such a side jet depends critically on its alignment relative to the capillary nozzle.? 


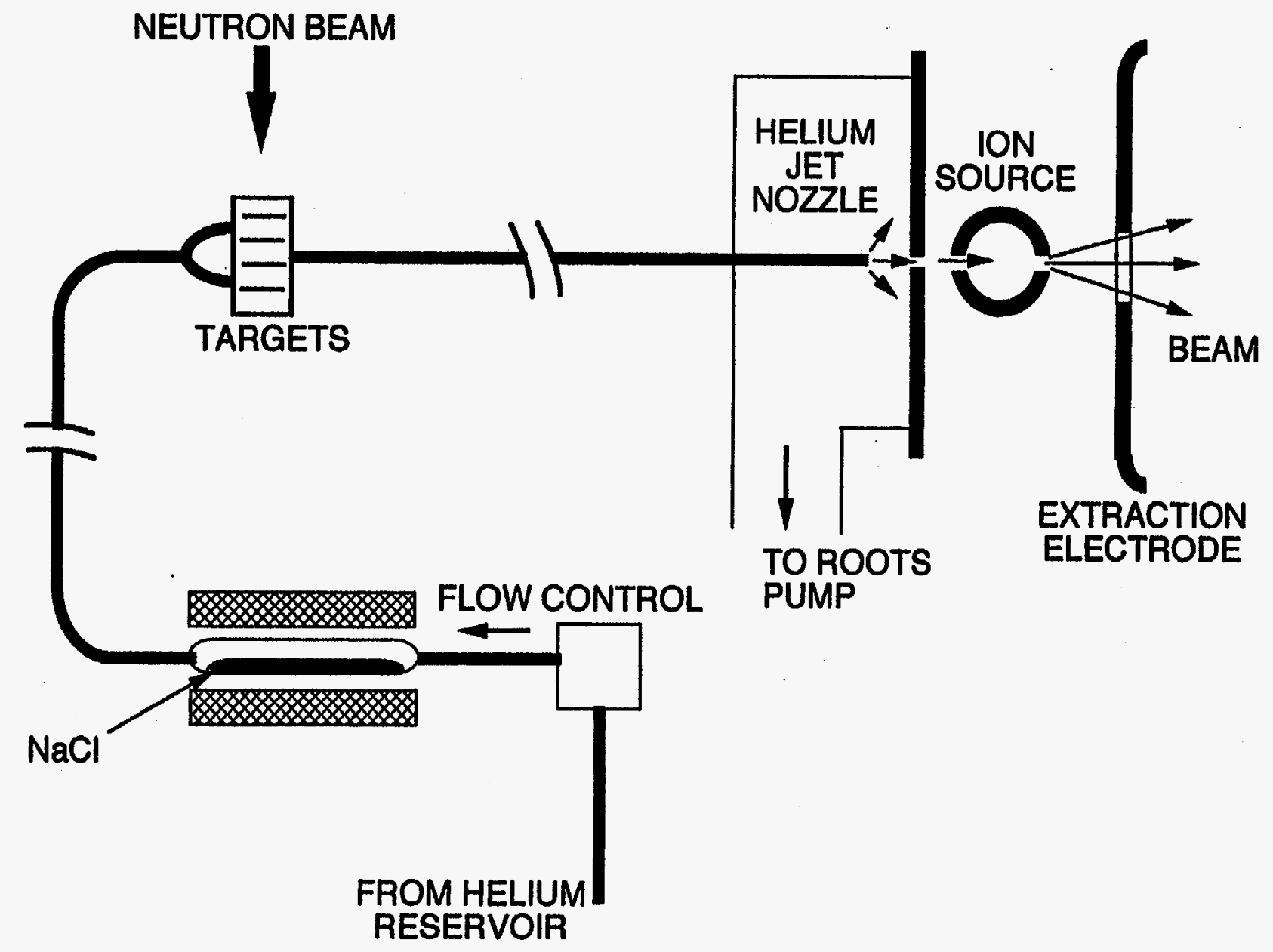

Fig. 3.14. A schematic diagram of the helium-jet transport system. Fission products attach to aerosol ( $\mathrm{NaCl}) \mathrm{clusters} \mathrm{in}$ the target chamber and are carried to the ion source. Most of the helium gas is removed by the skimmer between the jet nozzle and the ion source. 


\subsubsection{Operation of Material Transport Mechanism}

It is envisaged that the MTM will be operated only during reactor shutdown periods. This provision greatly reduces risk of accidental release of radiation from the LSBT.

\subsubsection{Loading of the Target Capsule}

The initial conditions assumed in the description of this operation are that the empty transport carriage is already at the top of the ball screw in the LSBT and that the rotary shutter mechanism is closed.

First, in a remote sample-preparation area, the new target capsule will be locked onto the capsuleloader mechanism. The combined capsule-loader assembly is then inserted into the shielded cask, which is closed, transported to the separator enclosure, and mounted on top of the rotary shutter mechanism (Fig. 3.2).

The rotary shutter is then moved to its fully open position, and the shielded cask is opened to provide direct access from the cask to the shutter. The capsule-loader assembly is then lowered into the shutter opening until it engages with the transport carriage. When in position, the capsule will engage with the transport carriage (via locking pins and housings), and the motor will engage with the ball screw (Fig. 3.11).

Next, the locking pins on the loader are disengaged to release the capsule so that it is now attached only to the transport carriage. The motor is then operated to rotate the ball screw so that the carriage-capsule assembly travels down the LSBT. The position and speed of the carriage can be controlled by the motor.

At the bottom of the ball screw, the carriage engages with the end stop (bottom ball-screw support) via locking pins and housings. At this point, automatic couplings on the capsule engage with the mating couplings on the ends of the capillary tubes (Fig. 3.7). Helium circulation through the capsule is then begun to verify the integrity of the circuit.

The shielded cask is closed, and the rotary shutter is moved to its fully closed position. The shielded cask is removed from the separator enclosure and monitored for contamination.

\subsubsection{Removal of the Target Capsule}

The initial conditions assumed in the description of this operation are that the target capsule is mounted onto the transport carriage and is located at the bottom of the ball screw in the LSBT and that the rotary shutter mechanism is closed. Active helium circulation through the capsule is also disabled.

First, an empty loader mechanism is loaded into the shielded cask, and the cask is closed. The cask is then transported to the separator enclosure and mounted on top of the rotary shutter mechanism.

The rotary shutter is then moved to its fully open position, and the shielded cask is opened to provide direct access from the cask to the shutter. The loader mechanism is then lowered into the shutter opening until it engages with the ball-screw drive-shaft coupling.

The motor is operated to rotate the ball screw. The resulting tension breaks the coupling (locking pins) of the transport carriage to the lower support and breaks the automatic coupling of the helium capillaries to the target capsule (Fig. 3.7). The motor is then operated to transport the carriage-capsule assembly to the top of ball screw.

At the top of the LSBT, the target capsule engages with the locking pins on the loader mechanism (Fig. 3.11). The pins are then locked, and withdrawal of the loader mechanism breaks the connection 
of the target capsule with the carriage mechanism (locking pins). The capsule-loader assembly is then withdrawn through the rotary shutter into the shielded cask.

The shielded cask is then closed, and the rotary shutter is moved to its fully closed position. The shielded cask is removed from the separator enclosure to a hot cell for monitoring and disposal of the irradiated target cell.

\subsubsection{Helium-Jet Ion Source}

\subsubsection{Design Considerations}

The primary consideration for the selection of an appropriate ion-source geometry is its capability of handling high gas throughput. It is expected that a source that normally requires a high throughput of feed gas would be least perturbed by the addition of helium from a skimmer system. A Bernas-Nier type source ${ }^{9}$ with slit geometry is an obvious choice because it offers a very favorable combination of throughput, source efficiency, and source emittance. Such a source (with a 1- $\times 30-\mathrm{mm}$ exit slit) has been shown to perform substantially better than a forced electron beam induced arc discharge (FEBIAD)-type ion source with a circular extraction orifice of $1 \mathrm{~mm} .^{8}$ The use of other, less-efficient ion-source geometries at certain helium-jet facilities can be attributed entirely to the inability of these facilities to handle the inherently high gas loads delivered by a slit geometry. ${ }^{10,11}$

Another important design consideration is a large ratio of extraction slit to entrance orifice area to prevent backstreaming of the fission products once they reach the source. A ratio of 1:10 is easily achievable, though in many existing helium-jet ion sources this ratio is actually less than 1 . In addition, the distance between the capillary nozzle and the ionization region should be minimized because of solid angle considerations. A typical distance between the skimmer and the source entrance orifice is $5 \mathbf{m m}$.

Beyond these considerations, consideration must also be given to minimizing the source volume, to operating with the source body at high temperatures, and to ensuring a high cold-spot temperature. All of these are general criteria for any ion-source design aimed at short holdup times, high source efficiency, and minimized deposition of material on insulating surfaces.

\subsubsection{Skimmer/Ion-Source/Pumping-Line Interface}

Because the body of the ion source must be operated at a high ( 30 to $70 \mathrm{kV}$ ) potential (and because there is a relatively high gas pressure in the small region between the skimmer and the ion source), any significant potential difference between the skimmer and the ion source will result in continuous discharge and possibly a general high-voltage breakdown. It is evident, then, that the skimmer must also be held at the same high potential.

However, this constraint merely transforms the problem to that of a possible continuous glow discharge in the pump line to the roots pump, which would be at ground potential. One possible solution to this problem is to introduce a multistage spark arrester into the pumping line ${ }^{8}$ (to decrease the potential in small, equal steps), but it has been found that even this approach cannot prevent breakdown at high helium flow rates. A simple solution to this obstacle is to operate the roots pump and associated fore-vacuum pumps at the same high potential as the ion source.?

\subsubsection{Ion Source}

Although it is possible that other ion source geometries could be used, this discussion focuses on the Bernas-Nier type, ${ }^{9}$ which has been shown to be applicable to helium-jet applications. ${ }^{7,8}$ 
The source body, which serves as the anode of the ion source, is a cylinder of 1 to $2 \mathrm{~cm}$ in diameter and 100 to $150 \mathrm{~mm}$ in length with an entrance orifice of $1.5 \mathrm{~mm}$ in diameter. The extraction slit on the back side of the ion source has typical dimension of $1 \times 30 \mathrm{~mm}$. The cathode assembly, separated from the source body by a ring of insulating material such as boron nitride, is inserted into one end of the cylinder and heated, either directly or by electron bombardment. To achieve source temperatures above $2400^{\circ} \mathrm{C}$, additional heating elements can be used. A spring-based mechanism compensates for thermal expansion of the ion-source body, and a locating pin maintains the alignment with the skimmer orifice. Concentric heat shields surround the source body, which is attached to a water-cooled copper back plate and further enclosed by water-cooled copper side plates.

For operation in plasma-discharge mode, a support gas, usually argon, is bled into the source, and arc discharge occurs between the cathode and anode. The source requires an axial magnetic field of 0.02 to $0.03 \mathrm{~T}$, which is supplied by an external electromagnet. Upon entering the plasma region, the aerosol particles break up and are ionized along with the transported fission products. The ions are then extracted, using an extraction electrode placed behind the exit slit, and delivered to the separator stage of the facility.

Efficiencies of 1 to $11 \%$ have been reported for such ion sources when operating in plasma discharge mode. ${ }^{8}$ When operated in surface-ionization mode (suitable only for alkali elements), efficiencies of better than $20 \%$ are possible. ${ }^{8}$ In such helium-jet ion-source systems, the target cell dwell time dominates any ion source or transit dwell time. Even so, total transit times, from production to extraction from the ion source, of well below $1 \mathrm{~s}$ are feasible.

It is evident that the previously given numbers for the fission source are maximum numbers. They would imply that efficient cooling of the source is ensured in all conditions (risk of meltdown of the target) and that almost all fission activity, about $20 \mathrm{kCi}$, is exported into the reactor hall. The final source design must be calculated for evacuation of the heat, failure of the helium gas flow, and the maximum admissible quantity of radioactivity attached to aerosols in the reactor hall.

Several problems are still subject to investigation within the scheme using the helium-jet approach. The loading of the helium with salt clusters of definite size and stable concentration is a procedure that is still in a prototype status. The coupling of the clusters to an ion source after pumping away the helium is a delicate task that may jeopardize the efficiency of the jet system. The next problem concerns the ionization and extraction efficiency of the ion source, for which information is not yet available. A remaining problem is the separation of the radioactive noble gases from the helium.

A serious drawback of the setup is the nonselectivity of the jet system coupled with its high efficiency. This leads to all the radioactivity (some $25 \mathrm{kCi}$ ) being transported out of the source and deposited in the experimental hall. Most of this activity will be deposited in the separator stage, though special provision would have to be made to trap noble gases (see below).

\subsection{IN-PILE BREEDER ION SOURCE}

\subsubsection{Concept}

Neutron flux values at the far end of the LSBT are predicted to be about $2.7 \times 10^{19} \mathrm{~m}^{-2} \cdot \mathrm{s}^{-1}$. This is about five times greater than those available at the high-flux reactor of the ILL and about three orders of magnitude higher than those found at existing medium-flux reactors. It is also about one order of magnitude higher than is expected at the planned new research reactor at Garching, Germany.

For in-pile positions at high-flux reactors, a few grams of ${ }^{235} \mathrm{U}$ leads to fission rates on the order of $10^{14}$ fission/s, which in turn leads to heating of about $6 \mathrm{~kW}$. If only radiation cooling is used, temperatures of $2400^{\circ} \mathrm{C}$ can be reached. (This is the temperature required for the fast release of 
short-lived fission products, and, in this case, no further means of heating or cooling of the source are necessary.) However, problems with such sources may arise because of the fast burnout of the uranium content. The half-life of such sources ranges from approximately three weeks to a few days when fluxes of $1 \times 10^{19} \mathrm{~m}^{-2} \cdot \mathrm{s}^{-1}$ are approached. For neutron fluxes below $5 \times 10^{18} \mathrm{~m}^{-2} \cdot \mathrm{s}^{-1}$ burnout compensation can be achieved by use of an antireactivity shield or by moving the source into higher neutron fluxes with time. It has been calculated that fission rates can be stabilized for about $120 \mathrm{~d}$ under these conditions.

At the ANS, neutron fluxes are such that the use of highly enriched ${ }^{235} U$ at the maximum flux position can be excluded for practical reasons because the burnout would be extremely fast-on the order of $4.5 \mathrm{~d}$. Also, it is not possible to compensate for this fast burnout with either an antireactivity shield or a source displacement.

However, the high neutron flux at ANS opens the way to a breeder solution for the ion source. In this approach the target material does not itself have a high fission cross section, but the neutron-capture reaction (and possibly subsequent beta decay) is used to "breed" a fissionable isotope. Depending on the various reaction rates involved, an equilibrium can be quickly attained, thus leading to a fission rate that is stable over a long time period.

\subsubsection{Choice of Breeder Materials}

The concept of a breeder source has considerable advantages in a high-flux reactor. The fission rate in saturation conditions is proportional to the breeder rate. With a sufficiently high cross section for fission, equilibrium conditions are rapidly achieved, and the proportionality factor is just 1 .

Breeding cross sections should be small to achieve a slow burnout rate of the source. For neutron fluxes at ANS, cross sections between 1 and $10 \mathrm{~b}$ are convenient. They allow for slow burnout and for reasonable amounts of material $(5$ to $15 \mathrm{~g}$ ) to be put into the ion source itself.

Two breeding reaction paths are possible. The first one is double neutron capture via an intermediate stable isotope. Reactions of this kind are ${ }^{231} \mathrm{~Pa}(2 \mathrm{n}, \mathrm{f}),{ }^{237} \mathrm{~Np}(2 \mathrm{n}, \mathrm{f})$, and ${ }^{241} \mathrm{Am}(2 \mathrm{n}, \mathrm{f})$. Here, lifetimes of the intermediate nuclei are long enough for the neutron-capture reaction to proceed directly to the fissioning compound nucleus. An interesting nucleus to investigate may be ${ }^{248} \mathrm{Cm}$, for which successive neutron capture leads to fission of both ${ }^{250} \mathrm{Cm}$ and ${ }^{251} \mathrm{Bk}$. Especially for fission products close to the magic tin shell, a considerable gain in intensity is expected in a thermal neutron source using this last reaction. However, all reactions proceeding via the $(2 n, f)$ path require the use of radioactive target material and would probably have to be considered at a later stage of the project.

Therefore, in the beginning, easy-to-handle sources should be considered, and the most natural choice would be either ${ }^{232} \mathrm{Th}$ or ${ }^{238} \mathrm{U}$. The amounts of material needed to reach fission activities on the order of $1 \times 10^{14} \mathrm{fission} / \mathrm{s}$ for these two are about 5 and $10 \mathrm{~g}$, respectively, which compare favorably with values currently used in thermal ion sources, either in in-pile positions or at external beams.

The burnout time of a ${ }^{232} \mathrm{Th}$ source would be about $400 \mathrm{~d}$ of reactor operation. The lifetime of the

${ }^{238} U$ source would be substantially longer $(1100 \mathrm{~d})$ because of the lower cross section. These long burnout times suggest that the breeder sources may stay in place for several years and would not have to be changed. The easiest solution would be to replace the fission source at the same time as the beam tube.

It is known that thorium and uranium atoms form stable chemical complexes with carbon at $2400^{\circ} \mathrm{C}$. However, a study would have to be made of whether protactinium and neptunium complexes will be built up (following thorium or uranium beta decay, respectively) in order to avoid large losses of the intermediate isotopes by diffusion out of the ion source.

If thorium is to be used, the fission product mass and charge distribution will be characteristic of ${ }^{233} \mathrm{U}$ fission with some admixture of ${ }^{235} \mathrm{U}$ fission. The fission yield characteristics obtained from such a 
source will be essentially the same as those currently obtained in other sources and thus will produce essentially the same fission products as those expected for the PIAFE setup.

If, however, breeding from ${ }^{238} \mathrm{U}$ is used, fission products will result from fission of the compoundnucleus ${ }^{240} \mathrm{Pu}$. In this case, the most likely fission product masses are shifted toward symmetry with respect to uranium. It is not expected that large gain factors will result for the yield of refractive elements between zirconium and palladium because these isotopes are known to be difficult to extract from a thermal source. However, an important feature is the increase of yield for symmetric fission, including silver, cadmium, and indium isotopes. For these elements, which are near the magic proton number 50 and neutron number 82 , it is expected that long isochains may be investigated on the neutron-rich side, thus leading to species far in excess of 17 neutrons above the stable configuration. In this respect, the use of ${ }^{238} \mathrm{U}$ in the breeder source would give access to new regions of fission product activity not currently accessible.

It should be noted, however, that if natural uranium is used, care must be taken to account for the ${ }^{235} \mathrm{U}$ content, which burns out rapidly and leads to a strong heating of the target arrangement with $\sim 12 \mathrm{~kW}$ at maximum flux.

It should be understood that the previous estimates for fission rate and burnout time are based on the assumption that thermal fission cross sections are used. Calculations at the ILL in Grenoble have shown that, depending on the source design, the neutron spectrum at the target position may harden considerably, causing the capture cross sections to decrease by as much as a factor of 2 in general. Final source designs will have to account for this shift in the neutron spectrum and the appropriate changes in cross sections.

\subsubsection{In-Pile Source Design}

The source itself will consist of a cylinder of porous graphite approximately $10 \mathrm{~cm}$ in length and $2.5 \mathrm{~cm}$ in diameter, with an inner cavity $-5 \mathrm{~mm}$ in diameter. The porosity of the graphite will allow the impregnation of $-10 \mathrm{~g}$ of breeder material. In order to avoid losses of fission products through the outer surface, the source must be sealed with a container of a highly refractive material, such as rhenium. The cylinder will be open to one side with an outlet orifice of 1 to $2 \mathrm{~mm}$ in diameter, and a heat shield should be used to minimize thermal conductivity away from the source. The whole is enclosed by a high-density graphite container to give the source mechanical stability and to allow for evacuation of heat by radiation to the beam tube. The exact dimensions of the source will be such that an equilibrium temperature of the fission source itself (inner shell) of about $2400^{\circ} \mathrm{C}$ can be reached in order to allow for the fast diffusion of short-lived fission products.

The source containment has to be maintained at a high potential of $-30 \mathrm{kV}$ to enable the extraction of the ionized fission products. Therefore, the gap between the outside of the source and the beam tube should be around 10 to $20 \mathrm{~mm}$. To hold the source in place, it will be mounted on a titanium tube $-2 \mathrm{~m}$ long. Titanium has been used for 20 years on the far end of the source changer to hold the fission source in place at the ILL fission product spectrometer Lohengrin. The neutron fluxes at that position are $\sim 5 \times 10^{18} \mathrm{~m}^{-2} \cdot \mathrm{s}^{-1}$. High-density graphite source holders have been used at the beta spectrometer at the $\mathrm{ILL}$ for the same period in fluxes near $3 \times 10^{18} \mathrm{~m}^{-2} \cdot \mathrm{s}^{-1}$.

A preliminary sketch of the thimble assembly, showing the source, the extraction plates, and two of the transport lenses (see Sect. 3.4.5) is shown in Fig. 3.15.

An insulator [made out of either aluminum oxide $\left(\mathrm{Al}_{2} \mathrm{O}_{3}\right)$ or beryllium oxide $(\mathrm{BeO})$ ] will be attached at the upper end of the titanium tube. The placement of the insulator at a distance of $-2 \mathrm{~m}$ from the far end of the beam tube ensures that the insulator is located in a considerably lower neutron flux and is much less subject to the influence of neutron capture, gamma heating from the core, and the continued deposition of evaporating fission products. The lifetime of insulators in the Lohengrin 


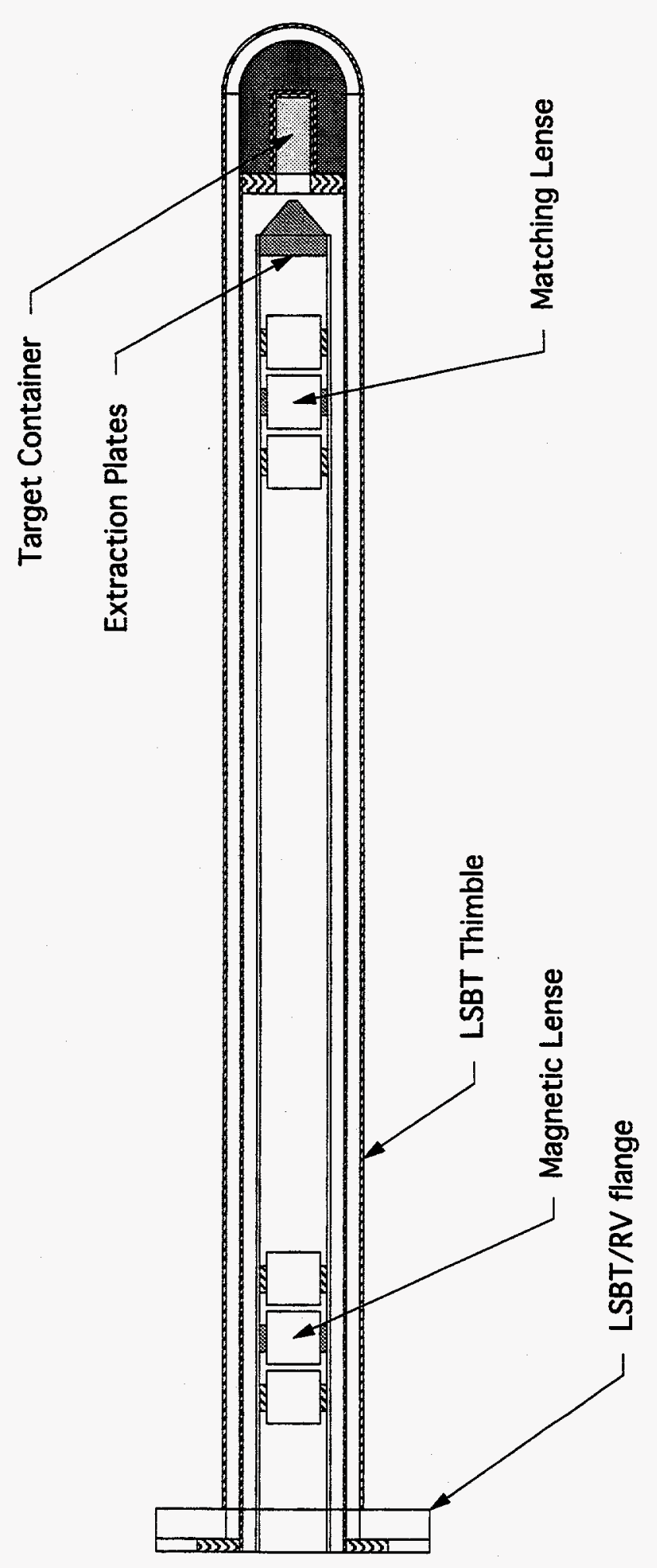

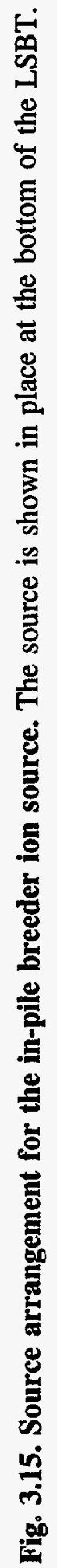


beam tube at ILL, which are located in a position where neutrons are almost absent, depends strongly on the vacuum conditions. Because pumping oils may crack under strong electric fields, an oil-free vacuum is required to avoid deposition onto the insulator surface. The lifetime of the Lohengrin insulators, with electric field values of above $1.2 \mathrm{gV} / \mathrm{m}$, is on the order of 3 to 4 years.

It is understood that the value given for the fission rate of the source is meant only as a guide. The power of $6 \mathrm{~kW}$ stems from the consideration that this power can be safely handled in a standard beam tube merely by using radiation cooling. The final layout of the source, its dimensions, and the number of heat shields will determine how strong the source can be made. The primary criterion is that the inner part of the source must reach a temperature of $-2400^{\circ} \mathrm{C}$ in order to facilitate the rapid extraction of exotic isotopes from fission. The power generated by the source is to be evacuated by radiation cooling only. The distance from the source container to the beam tube is to be $-1 \mathrm{~cm}$ to ensure a sufficient electrical insulation, which determines the maximum radius of the graphite container and, as such, in part determines its surface area, which is directly connected to the cooling rate.

Finally, it must be emphasized that the absence of forced heating (filament) or cooling (e.g., water, helium) constitutes the simplest approach to make the source largely maintenance free.

\subsubsection{Ionization Options}

\subsubsection{Surface and Electron-Impact Ionization}

To extract the fission products from the source electrostatically, they must first be ionized. The standard ionization procedure for fission products from in-pile sources is currently surface ionization on the extraction orifice, which leads to the selective extraction of rubidium and cesium isotopes with efficiencies of about $50 \%$ and barium and strontium at the $1 \%$ level. Any other elements can be extracted with only very small efficiencies.

At Studsvik, electron-impact ionization is used to ionize all elements in nuclear fission with efficiencies of about $\mathbf{0 . 1 \%}$. This leads to a considerable number of isotopes that are available at the focal position of the mass separator. However, electron-impact ionization is nonselective, meaning that large quantities of unwanted radioactivity are extracted, too.

Furthermore, the efficiency is low for any specific isotope. Also, electron-impact ionization demands (1) the positioning of a hot, electron-emitting electrode in front of the fission source and (2) the acceleration of the electrons into the source with an energy of $\sim 80 \mathrm{eV}$. This part of the extraction system must be considered as one of the elements leading to a limited lifetime of the setup.

\subsubsection{Laser Ionization}

Resonant laser ionization uses up to three laser frequencies to ionize selectively a particular element. (Because of Doppler broadening, selectivity is achieved for a given element, but not for specific isotopes.) Fast progress is currently being made in this area, and resonant levels have been established for the ionization of tin, thallium, and ytterbium with efficiencies at the $10 \%$ level. It is expected that resonant levels in other elements will be found soon, thus increasing considerably the range of isotopes that can be investigated. Additional heating of the source because of laser power is only a few watts and, therefore, negligible.

In nonresonant laser excitation, the frequency of the laser is unmatched, and multistep excitation proceeds via virtual levels. Ionization cross sections are 2 to 3 orders of magnitude lower. However, for future in-pile sources, this type of ionization may be of advantage because it leads to a maintenance-free ionization scheme, at least in the vicinity of high radiation near the source. The 
lasers themselves are located in the reactor hall in a radioactivity-free zone, about $14 \mathrm{~m}$ away from the source. Ionization over such distances is already standard at the isotope separator facility (Isolde) located at the Centre d'Etudes et Recherche Nucléaire (CERN) in Geneva, Switzerland. If, however, nonresonant ionization is to be achieved with high efficiency, the laser power may substantially contribute to the heating of the source. In turn this may be used to control the temperature of the fission source within a $10 \%$ temperature range.

The application of the resonant laser ionization scheme would be a substantial step forward for a highly efficient exotic beam facility. The increase in efficiency with respect to the Studsvik source and PIAFE would be a factor of $\sim 100$. Unwanted radioactivity is not ionized and, therefore, not extracted because it will stay near to the fission source itself. Ionization is maintenance-free in the neighborhood of the fission source itself; therefore, the lifetime of the fission source is not restricted because of the ionization step.

\subsubsection{Beam Transport in Pile}

The ion optical system to be positioned in the LSBT comprises the extraction electrode with a matching electrostatic lens and a periodical lens system in which identical Einzel lenses are placed $\sim 1.5 \mathrm{~m}$ apart in the beam tube. Each element of this periodic lens system is operated with the same high voltage, considerably decreasing the number of electrical feed-throughs and connections needed in the beam tube. A further study is needed to determine whether a second matching lens will be necessary in front of the first bending magnet (see below) to adjust the beam to the magnet aperture. The lenses will be made out of titanium, with the supporting insulators being either $\mathrm{Al}_{2} \mathrm{O}_{3}$ or $\mathrm{BeO}$.

Calculations have shown that such a periodical lens system can confine the ion beam on the tube axis within a diameter of $\sim 10 \mathrm{~mm}$, depending somewhat on the emittance of the extracted beam. Standard emittances are expected to be about $11 \pi \mathrm{mm} \mathrm{mrad}$, but the exact value depends on the ionization scheme, the outlet orifice, the temperature of the source, and the extraction voltage.

The distance $(\sim 10 \mathrm{~m})$ between the source and the bending magnet implies the need for about six electrostatic Einzel lenses with diameters of $\sim 80 \mathrm{~mm}$. With an extraction voltage of $30 \mathrm{kV}$ applied to the source, voltages on the Einzel lenses will be between 10 and $15 \mathrm{kV}$. Thus, there will be four different high voltages applied in the beam tube: one of $30 \mathrm{kV}$ for the source and three of $15 \mathrm{kV}$ for the electrostatic lens system.

The lens elements could be mounted into a large-diameter titanium tube at ground potential. Because of the thermal load of the tube at the lower end, axial dilatations are expected to be on the order of some centimeters. The crucial distance in the system is the gap between the source and the extraction electrode. This gap width must therefore be adjustable in the millimeter range, meaning that the whole optical system must be adjustable from the top end of the beam tube. Positioning of the setup should be on the order of $0.5 \mathrm{~mm}$. Radial dilatation plays a minor role, because of the axial symmetry of the setup.

Titanium can be used in high neutron fluxes for many years, as evidenced by the Lohengrin source changer at the ILL. Therefore, its use for the optical system implies that little or no maintenance will be necessary. The periodic lens system is shown in Fig. 3.16.

\subsubsection{Bending Magnet and First Mass Separation}

The LSBT is at an angle of about $58^{\circ}$. The ion beam must be deflected by this value to proceed in the horizontal plane to the separator stage either by electrostatic deflection with a mirror or by a bending magnet. The magnet solution has the advantage that, as well as deflection in the right direction, a first mass separation step is achieved. The angle of $58^{\circ}$ is convenient because it is near to 


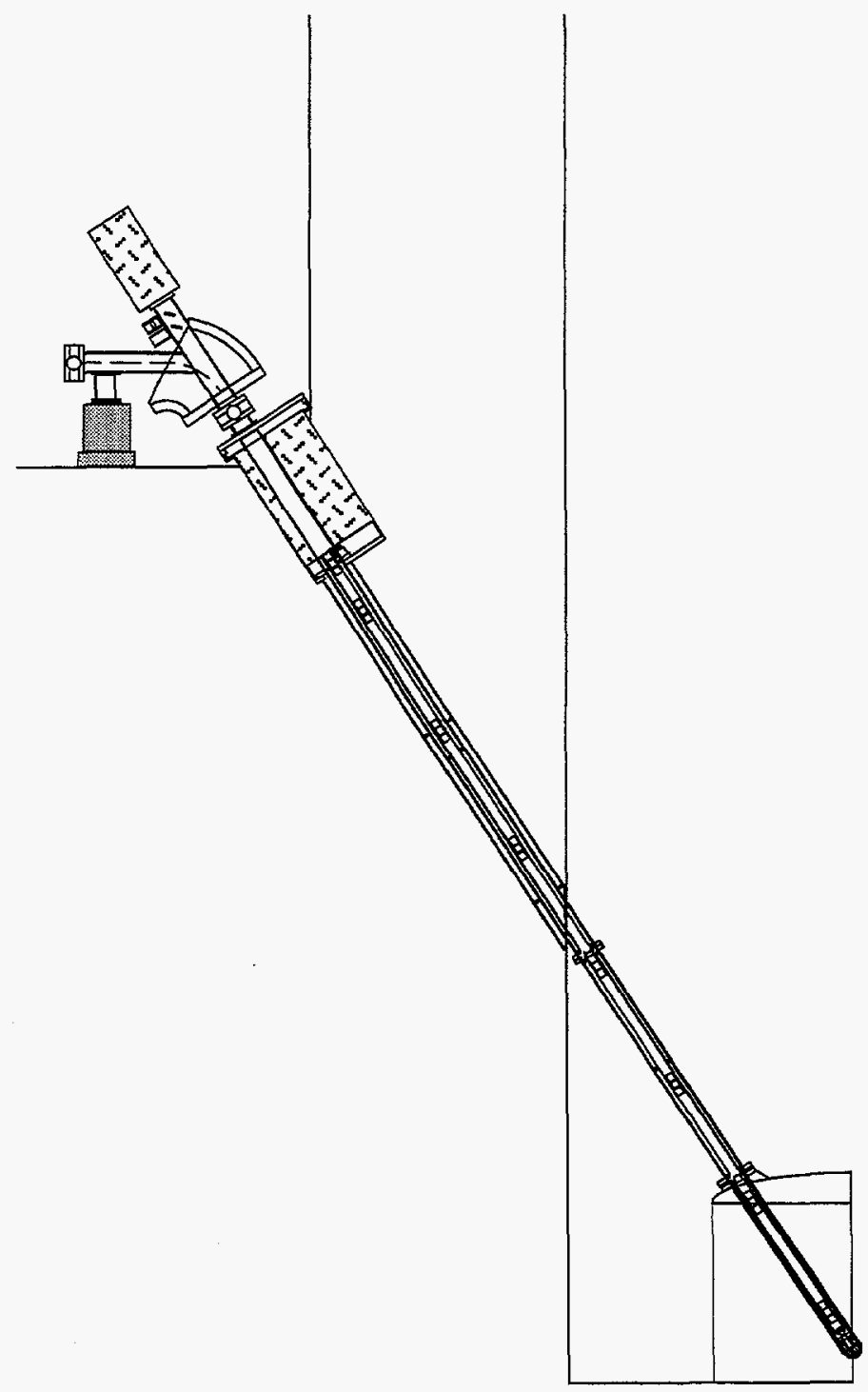

Fig. 3.16. A schematic diagram of the LSBT with the elements for use with the in-pile breeder ion source shown in place. The source itself is located at the lower end, and the periodic lens system is also shown, with the bending magnet at the top of the LSBT. 
the deflection of standard mass separators as used in other places: Isolde (CERN), Osiris (Studsvik), and PIAFE (Grenoble). If equipped with an edge at the exit side of about $35^{\circ}$, focusing is achieved at a distance of $\sim 900 \mathrm{~mm}$, and a diaphragm system can be used to perform a first mass separation.

The mass resolution of a bending magnet depends on the quality of the beam and the characteristics of the magnet itself. With a standard device, resolutions $(R=M / \delta M)$, between 700 and 1500 may be achieved, a value that is largely sufficient for a first mass separating step.

The dimensions of the magnet will depend on the bending radius and the gap width (distance between magnet poles). The bending radius of the magnet in standard devices is $\sim 700 \mathrm{~mm}$ for fission products of kinetic energy near $30 \mathrm{keV}$. The upper end of the LSBT has a diameter of $-200 \mathrm{~mm}$, which leads to a gap distance of $-250 \mathrm{~mm}$. Depending on the maximum beam size chosen at the magnet entrance (which, in turn, affects the mass resolving power), the length of the entrance slit will be $\sim 800$ to $1000 \mathrm{~mm}$ ( 4 times the gap width).

The choice of a large gap of $250 \mathrm{~mm}$ makes it easier to prevent neutron activation of the magnet and permits the easy extraction of the ion optical system if repair should be necessary. Because the magnet is to be installed on an inclined tube, its construction can be done in an H-shape. This shape reduces considerably the amount of iron needed for the magnet yoke and thus the weight of the device. Figure 3.16 shows how this magnet would be placed at the top of the LSBT.

\subsubsection{Parasitic Uses}

Parasitic use of additional masses on the mass separator is an option included at Isolde/CERN by use of an electrostatic deflection system behind the mass separating magnet. However, if an element-specific ionization process is chosen, the choice of parasitic masses will be restricted to the same element at ANS.

The dispersion of the bending magnet leads to distances between two neighboring mass lines of $\sim 7 \mathrm{~mm}$. Within one isotopic chain, some 8 masses are available from the fission process, located within a distance of $60 \mathrm{~mm}$. This appears to be too tight a space in which to place electrostatic separators. Moreover, the bending magnet deflecting vertically will not allow the separation of different masses in a horizontal plane.

\subsubsection{Radioactivity Levels}

Radioactivity values at the source position will depend on the type of breeder material used. Because of the relatively long lifetimes of intermediate actinides in the breeding process, it is expected that a series of transuranium isotopes will accumulate. Precise values need to be calculated with a burnup code.

Radioactivity from fission in a $6-\mathrm{kW}$ source amounts to about $25,000 \mathrm{Ci}$, mostly independent of what actinide is actually used. Whatever ionization scheme is chosen, most of this radioactivity will stay in the ion source itself. This is, however, not the case for the noble gases krypton and xenon, which will diffuse out of the fission source and reach the fore-pumps after a delay time of $\sim 5 \mathrm{~s}$. Halogens are also expected to diffuse out of the beam tube.

The radioactivity that will reach the diaphragm behind the first separator magnet is largely dependent on the ionization option. Nonselective ionization will allow a large fraction of the fission products to be ionized and to reach the mass separator. Depending on the setup, this activity may amount to values between 1 and $0.1 \%$, so that radioactivities on the order of $1000 \mathrm{Ci}$ are expected on the diaphragm behind the first mass separation. Nonspecific ionization leads to a high intensity of unwanted fission products in a given mass line. This activity will not be intercepted by the diaphragm in the focal plane behind the bending magnet and will propagate to the experimental area. 
If an element-specific ionization such as resonant laser ionization is applied, most of the background radioactivity will deposit on the diaphragm at the focal position behind the first bending magnet. The level of radioactivity at the diaphragm position will depend on the ionization process and the isotopes selected.

Resonant laser ionization may lead to a background ionization efficiency for all elements by nonresonant excitation at about $0.1 \%$ efficiency, which, in turn, leads to an activity of about $25 \mathrm{Ci}$ on the diaphragm. Tuned to a given element, the radioactivity at the diaphragm may reach values as high as $250 \mathrm{Ci}$. This is about a factor of 4 less than expected at PIAFE, where electron impact and surface ionization are responsible for high extraction rates over the whole fission product region.

\subsubsection{Noble-Gas Treatment}

Noble gases and part of the halogens will diffuse out of the source and will be pumped through the system. After a delay of $-5 \mathrm{~s}$, they will accumulate in the oil of the fore-pumps. Here about 95 to $98 \%$ of the radioactivity will be fixed. The remaining amount of radioactivity will go through the pumps and will have to be trapped. This is accomplished at CERN by stainless steel containers of $-5 \mathrm{~m}^{3}$ in volume. At the beginning of a cycle, these containers are evacuated to a primary vacuum and are subsequently filled with the pumped gases. After filling to a pressure of $\sim 200 \mathrm{kPa}$, the collected activity is allowed to decay before the gases are released through filters to the outside. Filling times are on the order of six months.

The fact that the fore-pump oils contain almost all of the total noble-gas radioactivity, amounting to about $2500 \mathrm{Ci}$, demands the installation of automated equipment to allow for the regular changing of the pump oils or for access to the pumping station when it is required for maintenance or repair. Because of the restricted access to the zone in which pumps and containers are located, all pumping stages should be designed to be redundant. Means should be foreseen to isolate a defective pumping unit and transfer its task to a spare unit.

Turbopumps will be used as secondary pumps, and standard rotary pumps will be used as fore-pumps. Roots pumps will be used to reach a pressure value of $200 \mathrm{kPa}$ in the storage containers. The outlets of the fore-pumps and the roots pumps must be vacuum-tight not to release gaseous fission products into the reactor containment hall. Because of the rather high values of radioactivity, the pumping units other than the turbopumps must be located in a shielded area.

\subsection{SEPARATOR DESIGN}

\subsubsection{Introduction}

Once the beam of ionized fission products is extracted from the ion source, the next stage is to isolate the desired mass for study. This is most easily done using a magnetic field of high intensity that deflects the particles in circular paths whose radii, in a given uniform magnetic field, depend on their mass-to-charge ratio $(M / q)$. Because the ions delivered by the ion sources discussed in this report are singly charged $(q=+1)$, for the vast majority, this stage amounts to a simple mass separation with the path radius $(r)$ determined by the mass $(M)$ of the ions and the magnetic field strength $(B)$. By using a sector magnet of fixed radius, different masses can be brought to a focus at different positions on an image plane after the magnet. 


\subsubsection{Performance Characteristics}

The performance of such a spectrometer can be discussed in terms of its resolving power $R=M / \delta M$, where $\delta M$ is the full width at half maximum (FWHM) of a peak at mass $M . R$ can also be expressed in terms of the dispersion $(D)$ as $R=D / m w$, where $m$ is the magnification of the separating magnet and $w$ is the object slit width. The dispersion is, in turn, given in terms of the displacement $d$ on the image plane, taken perpendicular to the beam axis, between focused ion beams of mass $M$ and $M+\Delta M: D=d(M / \Delta M)$. As a guide, a resolving power of $R=10$ is capable of effectively differentiating between only very light masses, whereas a value of $R>30,000$ would be capable of easily separating the different chemical species within one isobaric chain.

High resolving power can be achieved only through high dispersion, and this is proportional to the magnet's mean radius $r_{0}$. Unfortunately, the cost of constructing such a magnet scales approximately as $r_{0}^{3}$, whereas its performance is, within limits, proportional to $r_{0}$. Furthermore, the magnet gap has to be sufficiently high and wide such as to permit nearly complete beam transmission. Other considerations are the ability of the separator to handle high ion-beam currents, the range of isobaric masses for which it has reasonable mass resolution, and the overall transmission efficiency of the separated ions. In addition, any pumping system must be able to handle the increased gas loads inherent with a helium-jet ion source.

In reviewing the wide variety of separator designs currently in use, it is evident that two particular geometries offer superior performance. These will now be described.

\subsubsection{Sidonie-Type Separator}

This design of spectrometer, distinguished by its simplicity, was originally installed at Orsay ${ }^{12}$ and subsequently adopted for use at Chalk River. ${ }^{13}$ It involves a single separating magnet with a sector angle of $135^{\circ}$ and a mean radius of $\sim 1 \mathrm{~m}$. The distance between the ion-source orifice and the magnet entrance is also $\sim 1 \mathrm{~m}$. However, the beam shape is determined solely by the extraction geometry and can be optimized by moving the extraction electrode with precision drives that control four mechanical degrees of freedom (two translational and two rotational). Beyond this, there are no focusing lenses.

The gap between the pole pieces allows the use of a transport vacuum chamber $300 \mathrm{~mm}$ wide and $50 \mathrm{~mm}$ high. The simple geometry and large dimensions of the vacuum chamber mean that it can be easily accessed when components need changing or when the chamber must be decontaminated. This latter procedure can be made even easier with the use of a flexible liner inside the chamber. Care must be taken, however, to ensure that the vacuum chamber lines up with the optical axis of the separating magnet.

The separator beam travels through the $135^{\circ}$ magnet into a collection chamber. The image plane of the magnet crosses the collection chamber almost diagonally. The collection chamber is equipped with diagnostic equipment allowing fine-tuning of the separator field via correction coils mounted within the main magnet. In addition, equipment can be installed in the chamber to collect samples for off-line analysis. Separated beams can be guided through a narrow aperture in the image plane. This aperture defines the entrance to a beam transport line.

This separator has been shown to have excellent mass resolution even at very high beam currents $(20 \mathrm{~mA}) .^{13}$ Resolving powers of between 2500 and 8000 have been reported for masses up to $300 \mathrm{amu}$, and ion source to image plane transport efficiencies of up to $95 \%$ are also possible. The one big drawback of this system is that, because the mass of such a large magnet can be up to $16,000 \mathrm{~kg}$, provision must be made for this weight in the design of the second-floor beam room of the ANS. Figure 3.17 shows how an installation of a Sidonie-type spectrometer might be placed at the ANS. 


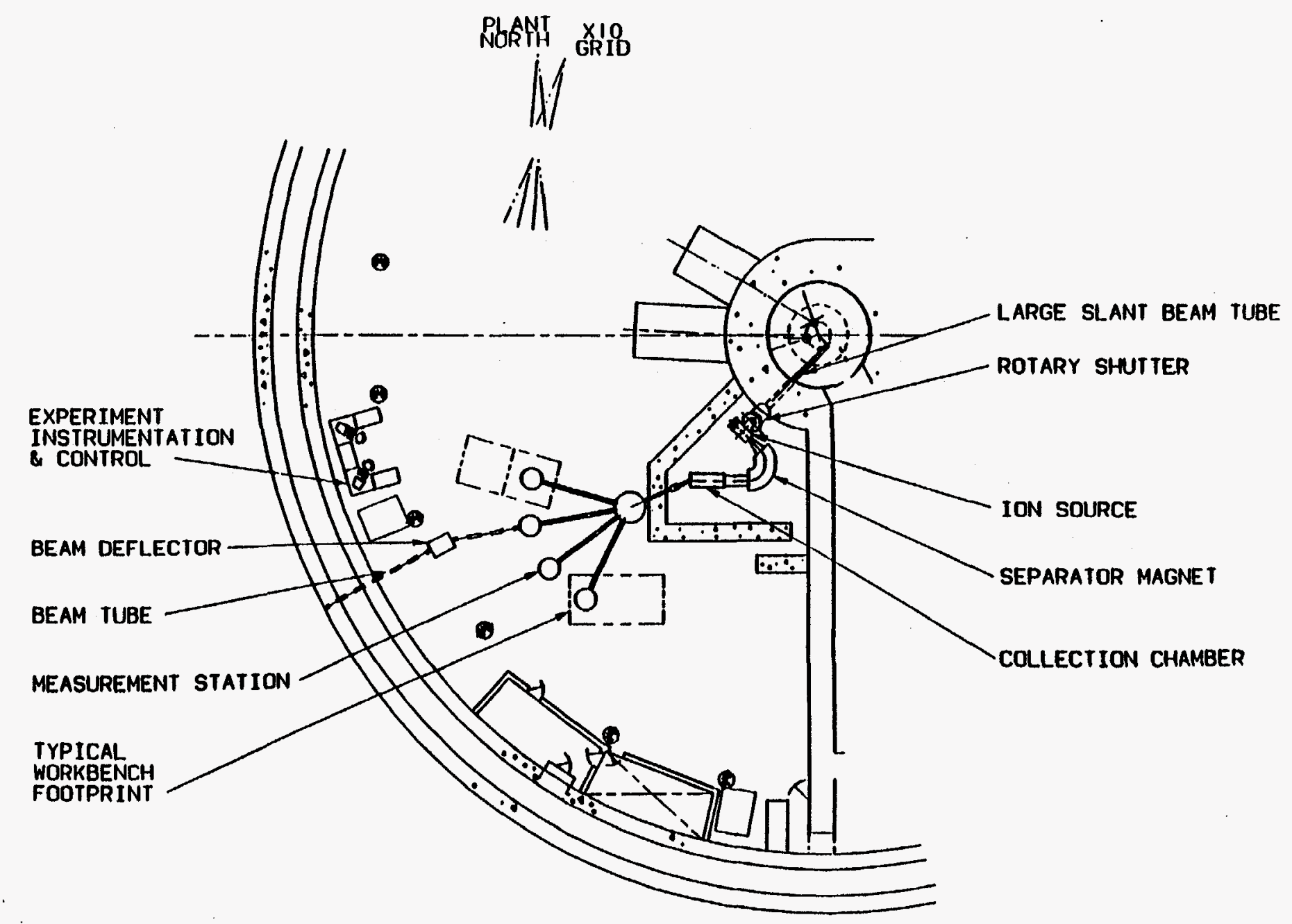

Fig. 3.17. A schematic layout of a Sidonie-type separator in the second-floor experimental area. The ion source, separator, and the collection chamber are in a shielded enclosure. The dashed beam line shows a future extension for delivering an ion beam to an external RIB facility. This beam line would penetrate the wall of the reactor building. 


\subsubsection{High Mass-Resolving-Power Separator}

The high mass-resolving-power separator ${ }^{14}$ takes a different approach in that it includes a high-current preseparator of low resolving power to remove the intense $\mathrm{He}^{+}$ion beam from the low-current radioactive beams of interest produced by a helium-jet ion source. This approach also allows more control over the ion-source plasma density and the shape of the plasma boundary. Ions of mass $\mathrm{A}=50$ to 250 are then passed into a two-stage separator consisting of two $90^{\circ}$ sector magnets, each with $1.5-\mathrm{m}$ mean radius and each preceded by an electrostatic quadrupole lens. The first stage will bring several ion masses into focus, but only one mass will be selected for transmission to the second stage by use of a narrow, interstage slit.

In order that the mass dispersions of the two separate stages add, they are arranged to deflect the ion beam in opposite directions, with an intermediate image between them. It has been calculated that such a separator could have a resolving power in excess of 30,000 . This is enough to separate different members of a single isobaric chain, a feat not achieved by any traditional separator designs. This feature would allow the study of nuclei with very low-production cross sections, even in the presence of very abundantly produced nearby nuclei.

\subsubsection{Focal-Plane Box, Switchyard, and Beam Lines}

All ion beams produced by the separator are brought to a focus some distance beyond the exit of the separator stage. This image plane will lie within a collection chamber (Fig. 3.17), allowing access to the separated ions at this point. Because several different mass chains can be brought to separate foci, an aperture at the focal plane allows the selection of the mass chain of interest.

Beyond this aperture lies an electrostatic deflector (switchyard), which is used to deflect the ion beam down one of four possible beam lines for delivery to a measurement station. Refocusing of the ion beam at the measurement station is achieved using a pair of electromagnetic quadrupole elements.

To facilitate the mounting and removal of experimental equipment while leaving enough space for more complex arrangements, a beam height of $\sim 1 \mathrm{~m}$ above the floor is desirable.

\subsection{MEASUREMENT STATIONS AND EQUIPMENT}

\subsubsection{Introduction}

In order to accommodate the variety of possible experiments that will be performed using the ISOL facility, four separate measurement stations are planned. Figure 3.17 shows how these measurement stations will be positioned, together with typical working area footprints and a possible location for experiment and instrument control. In addition to these stations, there will be access to the ion beam in the collection chamber at the focal plane position.

\subsubsection{Isotope Collection at the Focal Plane}

In the Sidonie-type separator (Sect. 3.5.3), several different mass chains can be brought to a focus within the collection chamber (Fig. 3.17) at different points on the focal plane. At these points, foils can be inserted to collect particular long-lived isotopes for later study. The mechanism for the insertion and removal of these foils will involve an interface between the high vacuum in the collection chamber and the outside environment. This can be achieved most simply with an intermediate chamber 
that can be evacuated or let up to atmosphere, as needed. Several ports on top of the collection chamber will be needed to access different regions of the focal plane. In the case of shorter-lived activity, these ports could be connected to a "rabbit" system for rapid transport of samples to a low background area for study.

Another possibility is to install a tape transport system (Sect. 3.6.4) at the focal plane to transport activity to a counting station located nearby. The advantage of having this option is that spectroscopy experiments could be carried out on one isotopic chain while another isotopic chain is passed on from the focal plane to the normal counting stations.

\subsubsection{Measurement Stations}

\subsubsection{Nuclear Spectroscopy}

It is expected that the main use for the isotope separator will be in the field of nuclear spectroscopy, so one measurement station will be reserved for this purpose. Typical studies will measure gamma-ray energies and intensities, gamma-gamma coincidences and correlations, fast-timing, conversion electrons, delayed neutron emission, and Q-values in $\beta$-decay. In all these cases the actual radiation detection will be performed using detectors from the central pool to be set up at ANS. These detectors will be mounted in a flexible framework at the counting site (on- or off-line). The detectors for particle decay will need to interface directly with the counting-chamber vacuum to avoid any losses in chamber windows or in the air. Each counting chamber must be designed to accommodate such ports.

\subsubsection{Measurements of g-Factors}

The measurements of nuclear g-factors require that the ion beam pass through a region of high magnetic field, supplied by a superconducting magnet. Because of the difficulty of removing and reinstalling such a magnet, a second measurement station will be dedicated to this apparatus.

\subsubsection{Laser Spectroscopy}

The most convenient method to measure the isotope shifts among different isotopes of the same element is by collinear laser spectroscopy. The sensitivity of the apparatus needed for this technique requires that it be permanently mounted on its own beam line. It is also preferable that this setup be contained in its own isolated chamber.

This beam line could also be used for ion-trap experiments, during which small numbers of ions are trapped and studied.

\subsubsection{Solid-State Physics}

The principle solid-state physics application for the separator will be the implantation of radioactive ions into solid matrix materials. These samples are then removed for later study off-line. Such implantations could be carried out at the focal plane, but better control of the beam spot and quality would be available at one of the counting chambers. A measurement station dedicated to ion implantation will mean that complex setups at other stations can be left in place and need not be disturbed for this relatively simple application. 


\subsubsection{Tape Transport Systems}

The isobaric chains produced by an isotope separator contain many different activities coming from sequential decays of the isobars in the separated mass chain. This phenomenon can greatly complicate matters when only one particular isotope is being studied.

To overcome this difficulty, a tape transport mechanism can be used. In such a system, a flexible tape intercepts the focused ion beam in the counting station, and the ions are collected on the tape. After a predetermined period, the tape is moved so that a new, clean portion of tape is brought into the counting chamber and the previously collected activity moves off to another location.

If the aim is to study short-lived isotopes, then the radiation detectors will be placed around the counting chamber, and the tape transport will be used to remove longer-lived daughter activity to a shielded location so that the activity does not interfere with the primary measurement. If the aim is to study one component of this daughter activity, then the radiation detectors will be mounted at a remote, off-line counting station. The tape cycle would then consist of a collection step, removal to an intermediate station to allow the short-lived activity to die out and, finally, delivery to the off-line counting station. The duration of collection cycles is determined by the lifetimes of the activities involved. The nuclear spectroscopy measurement station must be designed to interface with such a system. A tape transport system could also be used to collect separated ions at the focal plane in the collection chamber (Sect. 3.6.2).

For off-line fast-timing experiments, it is important that the activity spot be delivered to the same location (within a tolerance of $1 \mathrm{~mm}$ ) because the exact position of the source can affect the measurements. Such tape transport mechanisms are in common use at most separator facilities. To ensure precise placement of activities at remote stations, a special tape system has been developed at the University Isotope Separator at Oak Ridge. ${ }^{15}$ This system uses a combination of a light-emitting diode, a light sensor, and a tape with small holes in it to measure the exact movement of the tape.

\subsection{APPLICATION FOR RADIOACTIVE ION BEAMS}

As mentioned above, the ANS separator provides an opportunity to generate neutron-rich RIBs from the isotopes separated after neutron-induced fission. The selected isotope (or isobaric chain) would be passed from the focal plane through a beam tube to a remote location for re-ionization and then injection into a heavy-ion accelerator. The fact that the separator beam can be easily steered with the use of electromagnetic elements means that the beam could be transported long distances.

In the PIAFE project at Grenoble, ${ }^{4}$ this transport is accomplished via different successive steps: stopping the ion beam in a catcher foil, evaporation from the foil into the plasma of an electron-cyclotron resonance source, transformation to highly charged ions, injection into a first cyclotron, acceleration, extraction, stripping, injection into the second cyclotron, post-acceleration, and finally extraction of the particles with energies above the coulomb barrier. This scheme indicates that, although the fission source itself will be truly a second-generation device with ion currents of a specific isotope to be extracted of up to $1 \times 10^{12} \mathrm{particles} / \mathrm{s}$, the subsequent steps for acceleration are subject to intensity losses reaching up to 3 orders of magnitude.

The most convenient arrangement at ANS would be for the beam to exit through the reactor building at a point close to the separator, and for the re-ionization and acceleration to be located outside in a separate facility dedicated to this purpose. Therefore, provision has been made for a future extension of one of the central beam lines to pass through the wall of the reactor building (Fig. 3.17). This port should be in such a position that the beam will exit into a region reserved for the possible future installation of a RIB facility. The implantation of a LINAC near ANS would avoid the losses 
inherent in the PIAFE project and would make the installation a second-generation facility from the ion source design and from the acceleration scheme.

\subsection{CONCLUSIONS AND RECOMMENDATIONS}

\subsubsection{Targets and Ion Sources}

It is generally agreed that the conventional ion-source design option at ANS offers no new opportunities beyond those already available elsewhere. The same is certainly not true for the helium-jet ion source. However, this option does involve considerable risk in terms of interface with reactor systems and the expected delivery of large amounts of undesired radioactivity to the experimental area. Therefore, bearing in mind the expected rapid progress in laser ionization and the experience that will be gained with the PIAFE project in Grenoble, it is strongly recommended that an in-pile breeder ion source be considered for the ANS ISOL facility. Such an ion source offers the best opportunities for investigating new physics, while being considerably less complicated and easier to maintain than the helium-jet ion source.

In order to validate the approach for the new exotic beam source at ANS, several steps should be undertaken. First, the conditions for the in-pile breeder source have to be investigated by the verifying of source heating and equilibrium temperature conditions using well-developed and verified calculation procedures. Second, the conditions for radioactive-gas treatment must be fully elaborated.

The extraction and the electrostatic beam line can be tested by constructing a model and using a thermal rubidium ion source. The emittance and energy spread of the ion beam behind the $10-\mathrm{m}$ test tube can then be determined experimentally. In addition, the calculated reactor conditions may be approached by heating the test tube. It is also expected that the PIAFE setup in Grenoble will contribute considerably to the knowledge of conditions for the installation of an in-pile source in a high-flux reactor.

\subsubsection{Separator Design}

The Sidonie-type design is recommended for nuclear structure applications because it gives a combination of good resolving power and high beam current. The fact that several different isobaric chains can be brought to focus simultaneously may allow more than one experiment to be performed at a time. The mass of such a large separating magnet (and associated shielding) will need to be considered in the structural requirements for the second-floor beam room. The recommended height for the beam above the floor is $1 \mathrm{~m}$.

\subsubsection{Beam Lines}

Because some of the proposed experimental apparatus is sensitive to movement, it is recommended that four beam lines be established, with each dedicated to a specific application. Figure 3.17 shows a possible schematic layout for such a switchyard arrangement. Included is a branch that would leave the reactor building for delivery to an external RIB facility. A port will be provided in the reactor containment for this beam line. 


\section{DESIGN CONSIDERATIONS FOR THE THROUGH-TUBE}

\subsection{SOURCE-HOLDER ASSEMBLY DESIGN}

The tangential through-tube at ILL has been used successfully for high-precision gamma spectroscopy since its startup. ${ }^{16}$ Increasing demands for more mechanically stable sources led to a complete redesign of the source holder, which was installed in $1985 .{ }^{17}$ This design had great success in reducing mechanical source motion and in providing increased sensitivity in adjustment of the source position. This experience suggests that the source-holder assembly at the ANS facility should be based on the ILL design.

\subsection{SOURCE-CHANGING MECHANISM}

The ILL source-changing mechanism suffers from being very long and massive because of the continuous lead collimation, which must be inserted and withdrawn each time a source is to be changed. It can be shown that the same collimation could be achieved using only small collimators, one close to the source and one at the exit of the beam tube. This fact opens the way to two possibly simpler mechanisms:

- A small chariot carrying the sources and first collimator could be transported to the high-flux position. This would then be followed by the insertion of the second collimator at the exit of the beam tube.

- A multistage telescope-type arrangement could be used. In this way, sources would be loaded into the holder while the telescope is collapsed. The telescope would then be extended, section by section, until the source reaches the high-flux position.

A preliminary study of the ILL through-tube design does not preclude either of these options, but much further study would be needed to determine their feasibility.

\subsection{LOCATION}

At ILL, spectrometers are located at both sides of the tangential through-tube. At ANS the planned spectrometer would be on one side (T10), while the source-changing mechanism was originally planned to be on the other side (T9). However, a rearrangement of planned scattering instruments in the ground-floor beam room placed severe space restrictions on the T10 side while opening some space on the T9 side. For this reason, it was decided to relocate the spectrometer to the T9 side and place it behind the source-changing mechanism. Experience at $I L L$ shows that this would not be a serious problem. However, if one of the space-saving options for the source-changing mechanism discussed previously could be used, it is possible that it could be relocated to the T10 side. 



\section{DETECTORS FOR NUCLEAR SPECTROSCOPY}

\subsection{INTRODUCTION}

This section covers work in progress on the selection of detection systems for the nuclear and fundamental physics instruments within the ANS Project. Only the selection criteria for germanium (Ge) detectors have so far been defined in detail.

\subsection{NEED FOR DETECTORS AT ANS}

The detection and measurement of nuclear radiation will play a large part in experiments performed at several stations at ANS.

- The crystal spectrometer will measure gamma radiation (energy $<$ a few $\mathrm{MeV}$ ) from targets irradiated in the through-tube.

- The pair spectrometer will also measure gamma radiation (energies between 2 and $12 \mathrm{MeV}$ )

- Experiments at the nuclear spectroscopy stations will need to be able to measure $X$ and gamma rays, electrons (from $\beta$-decay and internal conversion), neutrons (fast and slow), and charged particles such as protons, alphas, and fission fragments.

- Similar capabilities will be needed at the nuclear orientation facility and at the ISOL.

- Fundamental physics experiments will also need access to radiation detectors for certain experiments.

In many cases, several detectors will be used simultaneously to detect coincidences between different radiations.

The detectors needed to accomplish these tasks fall into two categories:

- Generic, off-the-shelf devices that are commercially available would form a general pool from which individual detectors would be taken depending on the experiment configuration desired. An obvious example of a detector type falling into this category is the germanium detector, which is a standard for measuring the energy and intensity of $\mathrm{X}$ and gamma radiation.

- Dedicated instruments designed for a particular purpose and location that would, in general, have to be custom built. An example might be a magnetic spectrometer for the analysis of conversion and beta-decay electrons.

\subsection{GENERAL DETECTOR POOL}

\subsubsection{Gamma- and X-Ray Detection}

\subsubsection{Performance Characteristics}

In rating the performance of gamma detectors, it is common to consider four aspects:

1. The energy resolution at a given energy, which is expressed as the FWHM of the statistical distribution of peak heights resulting when monoenergetic photons deposit all of their energy in 
the detector. The standard photon energies usually chosen for this are $5.9 \mathrm{keV}$ (manganese $\mathrm{K}$ $\mathrm{X}$ rays from a ${ }^{55} \mathrm{Fe}$ source), $122 \mathrm{keV}\left({ }^{57} \mathrm{Co}\right)$, and $1332 \mathrm{keV}\left({ }^{60} \mathrm{Co}\right)$. For scintillation detectors the resolution is normally expressed as a percentage of the photon energy, whereas for semiconductor detectors an absolute value is usually given.

2. The detector efficiency measures the fraction of monoenergetic photons emitted by a point source that deposit their full energy in the detector. Thus the efficiency is geometry dependent. Standard practice is to express a detector's efficiency (as a percentage) relative to that of a 76.2-mm-diam $x$ $76.2-\mathrm{mm}$-long $\mathrm{NaI}(\mathrm{Tl})$ crystal if both were placed $25 \mathrm{~cm}$ from the photon source. [The absolute efficiency of such an $\mathrm{NaI}(\mathrm{Tl})$ crystal, in this geometry, is known to be $1.2 \times 10^{-3}$.] It must be noted that the efficiency is both geometry and energy dependent.

3. The peak shape is often specified in terms of the ratio of its full width at a tenth of the maximum height (FWTM) to the FWHM. For an ideal Gaussian response, this ratio should be 1.82 .

4. When a photon interacts inside a detector, it may be Compton scattered. If the scattered photon then leaves the detector, it is evident that only a part of the original incoming energy is deposited. This gives rise to a continuous Compton background at energies lower than the full energy of the original photon. A further characteristic of a detector's performance is the ratio of Compton scattering events that deposit only a part of the original photon energy to the number of events that leave the full photon energy in the detector, known as the peak-to-Compton ratio.

These performance characteristics are not independent. For instance, a larger detector volume will have a higher efficiency and a better peak-to-Compton ratio simply because there is more detector material available in which photons can interact. However, it is generally true that a larger volume detector will be less efficient in terms of charge (or light) collection, and so its energy resolution will be slightly worse.

\subsubsection{Selection Criteria}

Several criteria need to be considered in the selection of photon detectors.

- Detector type. For most applications, energy resolution is the most important factor, and in this case a semiconductor detector should be chosen. For some applications (such as fast timing), the poor energy resolution of scintillation detectors is more than compensated for by their superior timing characteristics. For scintillation detectors, the main choice is in what scintillation material to use. The selection of an appropriate photomultiplier tube is then based upon this choice.

- Detector material. For semiconductor detectors, the choice is among $\mathrm{Si}(\mathrm{Li})$ (which is better for energies below $20 \mathrm{keV}$ ), a p-type $\mathrm{Ge}$, or an n-type $\mathrm{Ge}$. Because of their thin frontal layer, n-type $\mathrm{Ge}$ detectors are able to measure both high- and low-energy regions. They are also more resistant to damage from fast neutrons, although they are usually more expensive than the other choices.

- Detector configuration. Because semiconductor detectors require constant cooling, they are usually coupled to dewars of liquid nitrogen. The configuration of the dewar assembly is determined by the space available in the experimental setup.

- Sensitive volume. The volume and cross section of the detector will depend on the expected use of the detector. For the detection of low-energy photons, it is better to choose a thin detector so that high-energy photons can pass straight through it without interacting. For the detection of high-energy photons, it is often desirable to choose the largest volume detector available, though obviously it will also be the most expensive. 
- Window material and thickness. When detecting low-energy photons, it is desirable to minimize their absorption in the inert detector housing. To this end, such detectors are equipped with thin windows of low-absorption material, usually beryllium.

- Detector energy resolution and response. Within small variations, most similar types of detectors will have similar energy resolutions (FWHM). It is known that the resolution of semiconductor detectors gets slightly worse with increasing volume, but this is not a very significant effect. However, at very low energies $(<10 \mathrm{keV})$ the resolution of an n-type Ge detector can be 3 to 4 times worse than that of a $\mathrm{Si}(\mathrm{Li})$ or planar $\mathrm{Ge}$.

The deviation of a detector's response from the ideal Gaussian response function can provide problems when it comes to analyzing experimental data. For a true Gaussian, the FWTM/FWHM ratio should be 1.82, whereas real detectors generally have ratios of between 1.9 and 2.0 .

The peak-to-Compton ratio is determined largely by the detector size and resolution and is usually a secondary consideration in detector selection.

\subsubsection{Ge Detectors}

The most convenient detector for $\mathrm{X}$ and gamma rays is a large germanium $\mathrm{p}-\mathrm{n}$ junction diode, combining high relative efficiency ( 20 to $120 \%$ ) with good resolution. Photons deposit energy in such a detector by either the photoelectric effect, Compton scattering, or $\mathrm{e}^{+} \mathrm{e}^{-}$pair creation. In principle, all these processes result in the photon energy's being transferred to electron-hole pairs in the Ge crystal. These charges are then collected at the surface electrodes of the crystal, and the size of the current that then flows is determined by the energy deposited. Efficient charge collection is accomplished by applying a large reverse bias to the crystal. 



\section{REFERENCES}

1. Nuclear and Fundamental Physics Instruments, ORNL/ANS/NNT-26/SDD-43, Martin Marietta Energy Systems, Inc., Oak Ridge Natl. Lab., May 1991 (draft).

2. Nuclear and Fundamental Physics Instruments, ORNL/ANS/NNT-26/SDD-43/RA, Martin Marietta Energy Systems, Inc., Oak Ridge Natl. Lab., June 1993.

3. H. Faust, Exotic Isotope Production from Nuclear Fission, 94FA1T, Institut Laue-Langevin, Grenoble, France, 1994.

4. H. Faust et al., Principles on the Implontation of a Thermal Ion Source in the H9 Beam Tube of the ILL, 93FA9T, Institut Laue-Langevin, Grenoble, France, 1993.

5. H. Faust et al., Proposal for an Exotic Beam Facility at the ILL, 94FA2T, Institut Laue-Langevin, Grenoble, France, 1993.

6. W. L. Talbert et al., "Optimization of a He-Jet Activity Transport System to Use at LAMPF," Nucl. Instrum. Methods B26, 345 (1987).

7. V. T. Koslowsky et al., "The Chalk River High-Temperature Helium-Jet Ion Source," Nucl. Instrum. Methods B70, 245 (1992).

8. H. Schmeing et al., "The Chalk River Helium-Jet Ion Source," Nucl. Instrum. Methods B26, 321 (1987).

9. J. Chavet and R. Bernas, "A New Type of Ion Source with High Emmitance," Nucl. Instrum. Methods 51, 77 (1967).

10. F. B. Blönnigen et al., "Improvement to the Helium-Jet Coupled On-Line Mass Separator RAMA," Nucl. Instrum. Methods B26, 328 (1987).

11. Y. Kawase et al., "Mass Separation of Rare-Earth Elements by a High-Temperature Thermal Ion Source Coupled with a He-Jet System," Nucl. Instrum. Methods B26, 341 (1987).

12. J. Camplan et al., "Sidonie, the New Electromagnetic Isotope Separator at Orsay," Nucl. Instrum. Methods 84, 37 (1970).

13. H. Schmeing et al., "The Chalk River On-Line Isotope Separator," Nucl. Instrum. Methods 186, 47 (1981).

14. W. L. Talbert et al., "Ion-Optical Design for an On-Line Mass Separator at LAMPF," Nucl. Instrum. Methods B26, 351 (1987).

15. P. K. Joshi et al., "Picosecond Lifetime Measurement Systems for Levels Populated by Electron Capture and for Levels Decaying by E0 Transitions," Nucl. Instrum. Methods A335, 461 (1993).

16. H. R. Koch et al., "The Bent-Crystal Spectrometers GAMS 1 and GAMS 2/3," Nucl. Instrum. Methods 175, 401 (1980).

17. F. Hoyler et al., "Recent Developments at the ILL (n,y) Facility," Inst. Phys. Conf. Ser. 88, 161 (1988). 

Appendix A

JUNE 1993 SDD-43 



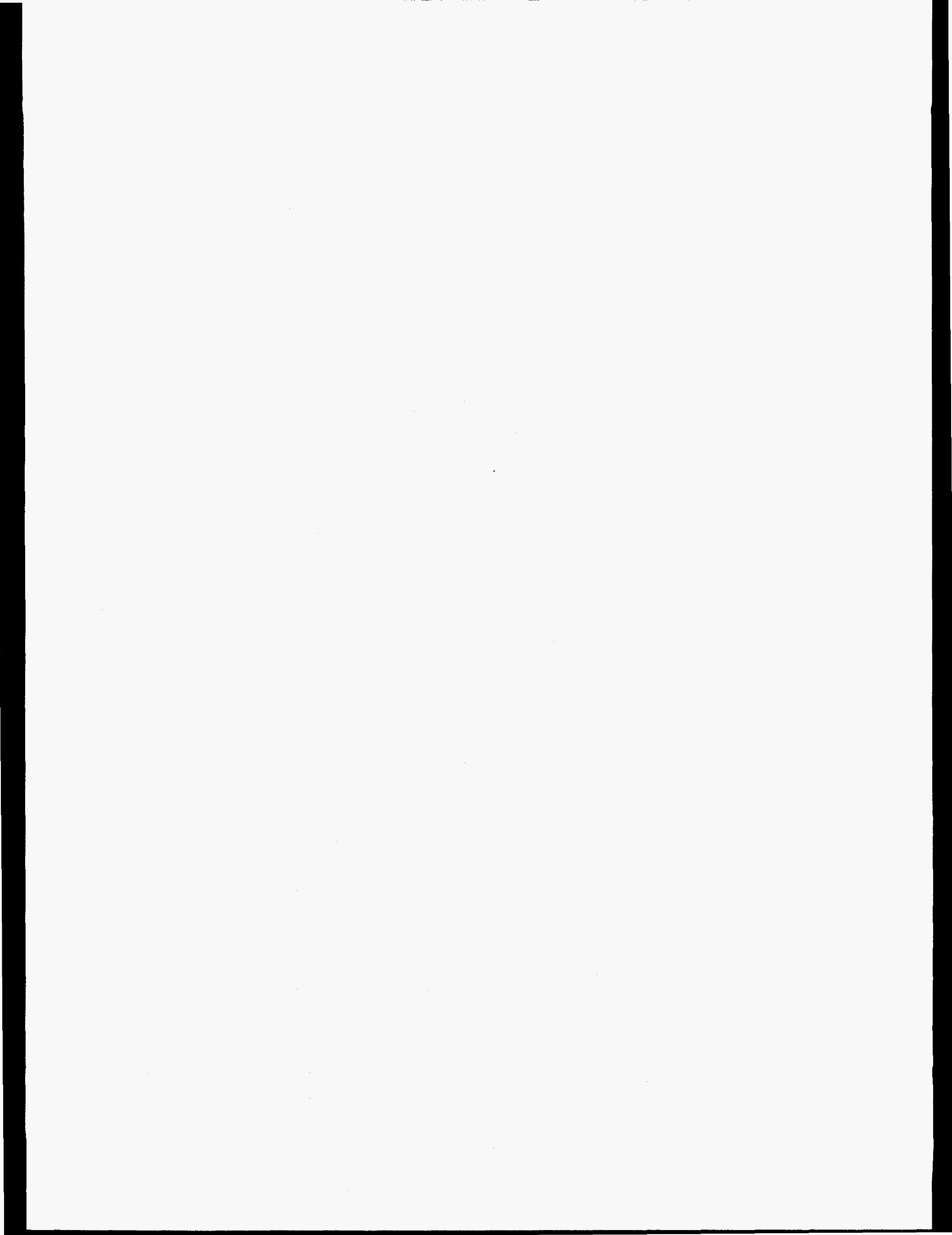




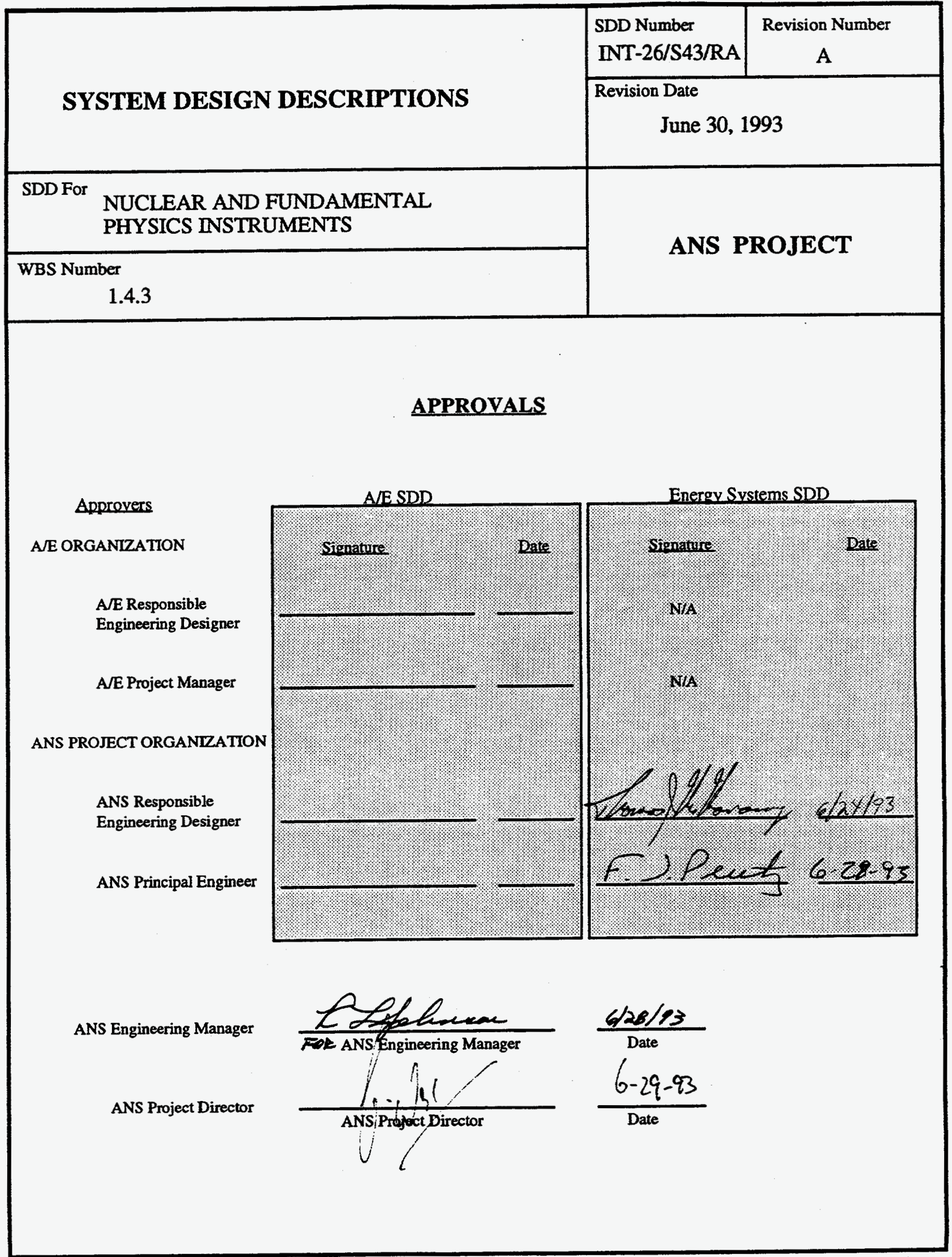




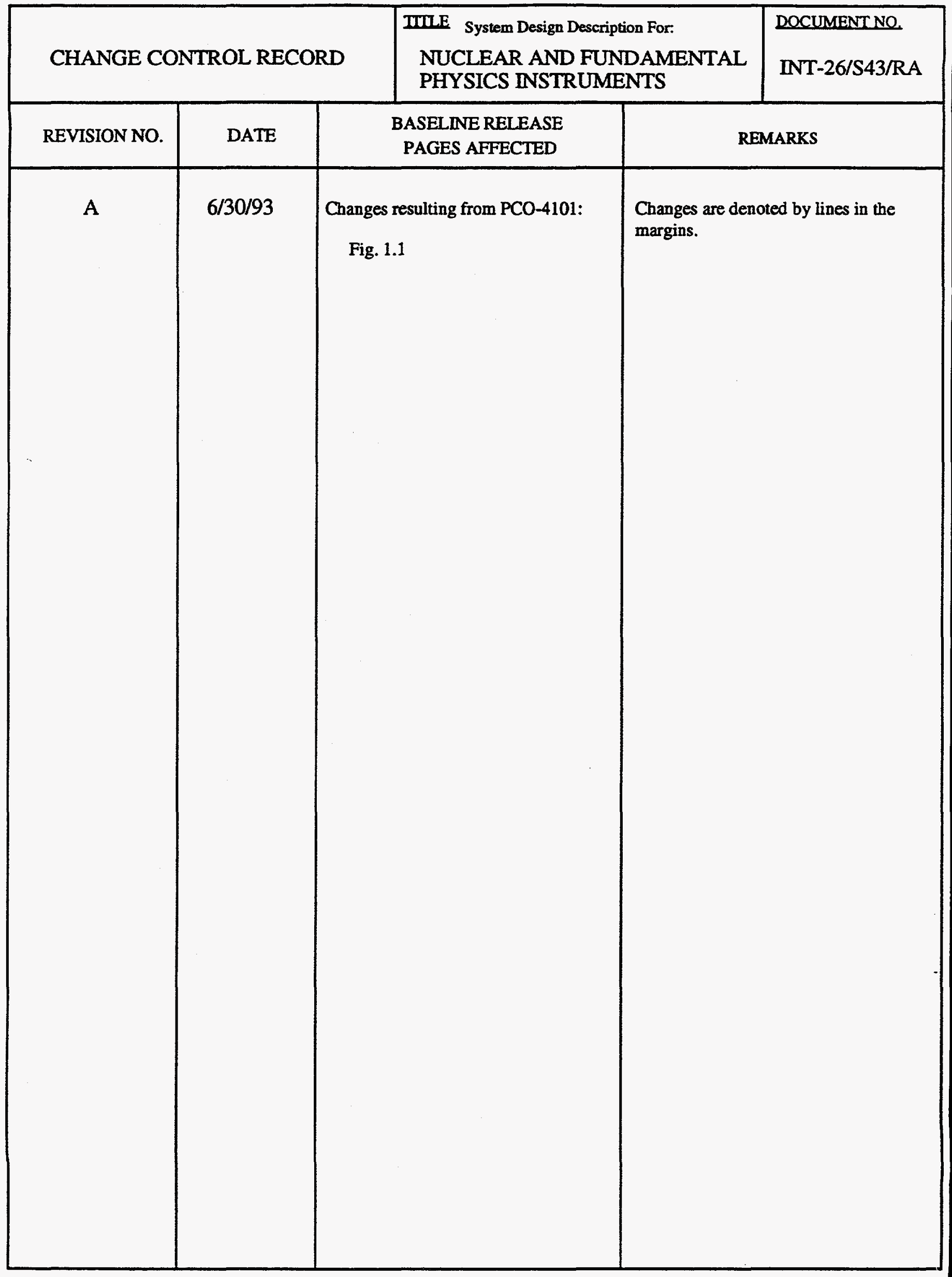




\section{ADVANCED NEUTRON SOURCE PROJECT SYSTEM DESIGN DESCRIPTION}

This document is an integral part of the total documentation prepared in support of the design of the Advanced Neutron Source (ANS) Project. A complete list of the System Design Descriptions (SDDs) and the Integrating System Design Descriptions (ISDDs) planned for the ANS Project appears on the next page.

This SDD was initiated as part of the conceptual design documentation for the ANS Project. Section 1, "Funetions and Design Requirements," of this SDD was approved as "Baseline" at the time of issuance of the Conceptual Design Report with the remaining sections of the SDD issued "Approved" but not under baseline control. As design work progresses, this SDD will incorporate more information, with periodic releases planned throughout the life of the project. At each release, an appropriate change documentation entry will be made to the Change Control Record page of this SDD. This record will include identification of baseline and nonbaseline changes.

Development of this SDD is an ongoing process. Users of this document are encouraged to contact the ANS Project Office to obtain the latest information on the status of this SDD.

Advanced Neutron Source Project Office

Fusion Engineering Design Center, Mail Stop 8218

104 Union Valley Road

Oak Ridge, Tennessee, 37831-8218

Phone: 615/574-0558 (FAX 615/576-3041) 


\section{Integrating Systems:}

21 Reactor containment systems

22 Reactor shutdown and cooling systems

23 Environmental compliance and monitoring

24 Security functions

25 Instrumentation, control, computing, and telecommunications systems

\section{Reactor Systems:}

31 Reactor assembly

32 Refueling system

33 Reactor instrumentation and control

34 Fuel element assembly

35 Reactor assembly mockup

36 Reactor maintenance systems

\section{Experiment Systems:}

41 Neutron beam transport

42 Neutron scattering instruments

43 Nuclear and fundamental physies instruments

44 Transuranium production facilities

45 Materiais irradiation facilities

46 Isotopes production facilities

47 Analytical chemistry facilities

48 Instrument support facilities.

49 Experiment systems computer network

410 Cold source assemblies

411 Hot source assembly

Site and Buildines:

51 Land improvements

52 Reactor building

53 Reactor support building/operations suppor building

54 Guide hall/research support building

55 Office building/interface building

56 Detritiation building

57 Other structures

58 Construction support

\section{Plant Systems:}

61 Reactor water systems

62 Plant electric power and communication systems

63 Environmental control systems

64 Plant water systems

65 Plant services systems

66 Plant fire protection

67 Plant waste systems

68 Heavy water upgrade and detritiation systems

69 Plant instrumentation, control, and data systems

610 Plant maintenance and general purpose equipment 


\section{CONTENTS}

$\underline{\text { Page }}$

LIST OF FIGURES $\ldots \ldots \ldots \ldots \ldots \ldots \ldots \ldots \ldots \ldots \ldots \ldots \ldots \ldots \ldots \ldots \ldots \ldots$

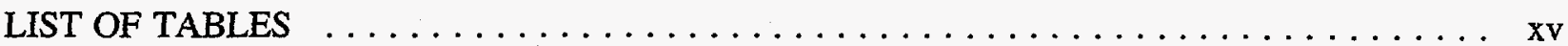

ACRONYMS $\ldots \ldots \ldots \ldots \ldots \ldots \ldots \ldots \ldots \ldots \ldots \ldots \ldots \ldots \ldots \ldots \ldots \ldots \ldots$

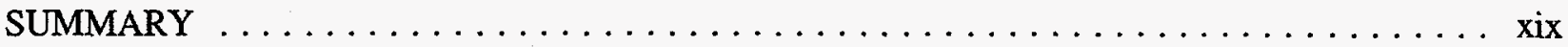

1. FUNCTIONS AND DESIGN REQUIREMENTS $\ldots \ldots \ldots \ldots \ldots \ldots \ldots \ldots \ldots \ldots \ldots$

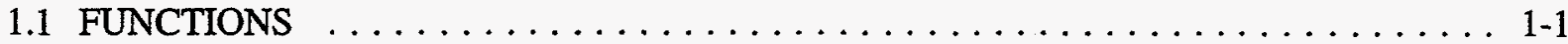

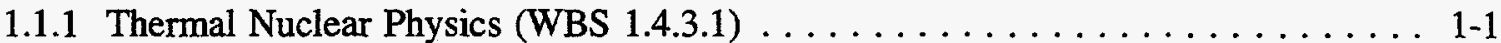

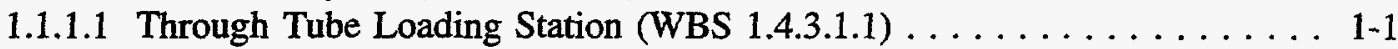

1.1.1.2 Ultrahigh Resolution $\gamma$-Spectrometer (WBS 1.4.3.1.2) . . . . . . . . . 1-1

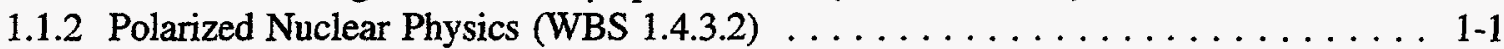

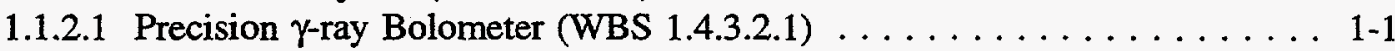

1.1.2.2 Weak Interaction Physics (WBS 1.4.3.2.2) $\ldots \ldots \ldots \ldots \ldots \ldots \ldots \ldots$ 1-1

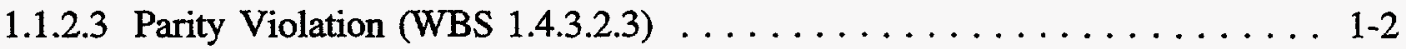

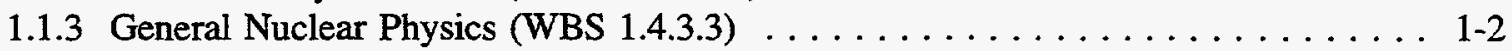

1.1.3.1 Nuclear Structure (WBS 1.4.3.3.1) . . . . . . . . . . . . . . 1-2

1.1.3.2 Nuclear Spectroscopy (WBS 1.4.3.3.2) . . . . . . . . . . . 1-2

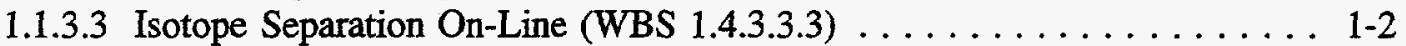

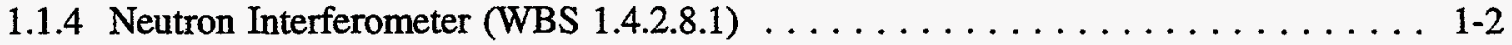

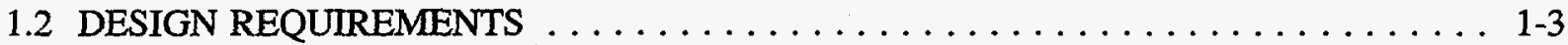

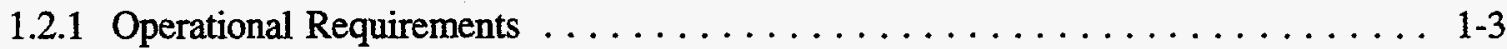

1.2.1.1 Thermal Nuclear Physics (WBS 1.4.3.1) . . . . . . . . . . . 1-3

1.2.1.1.1 Through tube loading station (WBS 1.4.3.1.1) . . . . . . 1-3

1.2.1.1.2 Ultrahigh resolution $\gamma$-spectroscopy (WBS 1.4.3.1.2) $\ldots \ldots \ldots 1-3$

1.2.1.2 Polarized Nuclear Physics (WBS 1.4.3.2) $\ldots \ldots \ldots \ldots \ldots \ldots \ldots \ldots$. . . . . . . . .

1.2.1.2.1 Precision $\gamma$-ray bolometer (WBS 1.4.3.2.1) . . . . . . 1-3

1.2.1.2.2 Weak interaction physics (WBS 1.4.3.2.2) . . . . . . . 1-4

1.2.1.2.3 Parity violation (WBS 1.4 .3 .2 .3$) \ldots \ldots \ldots \ldots \ldots \ldots . \ldots \ldots$

1.2.1.3 General Nuclear Physics (WBS 1.4.3.3) . . . . . . . . . . . 1-4

1.2.1.3.1 Nuclear structure (WBS 1.4.3.3.1) . . . . . . . . . . 1-4

1.2.1.3.2 Nuclear spectroscopy (WBS 1.4 .3 .3 .2$) \ldots \ldots \ldots \ldots \ldots$. . . . . . . .

1.2.1.3.3 Isotope separation on-line (WBS 1.4.3.3.3) $\ldots \ldots \ldots \ldots$. . . .

1.2.1.4 Neutron Interferometer (WBS 1.4.2.8.1) . . . . . . . . . . . . . . . 1-4

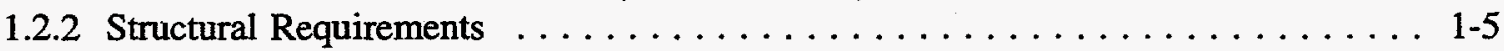

1.2.2.1 Thermal Nuclear Physics (WBS 1.4.3.1) . . . . . . . . . . 1-5

1.2.2.1.1 Through tube loading station (WBS 1.4.3.1.1) . . . . . . 1-5

1.2.2.1.2 Ultrahigh resolution $\gamma$-spectroscopy (WBS 1.4.3.1.2) $\ldots \ldots \ldots 1-5$

1.2.2.2 Polarized Nuclear Physics (WBS 1.4.3.2) $\ldots \ldots \ldots \ldots \ldots \ldots \ldots$. . . . . . . .

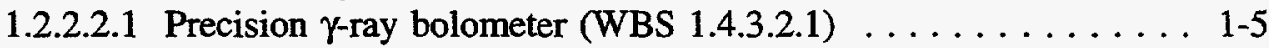

1.2.2.2.2 Weak interaction physics (WBS 1.4.3.2.2) . . . . . . . 1-6

1.2.2.2.3 Parity violation (WBS 1.4 .3 .2 .3$) \ldots \ldots \ldots \ldots \ldots \ldots$ 
1.2.2.3 General Nuclear Physics (WBS 1.4.3.3) . . . . . . . . . . 1-6

1.2.2.3.1 Nuclear structure (WBS 1.4.3.3.1) . . . . . . . . . . 1-6

1.2.2.3.2 Nuclear spectroscopy (WBS 1.4.3.3.2) . . . . . . . . . . 1-6

1.2.2.3.3 Isotope separation on-line (WBS 1.4.3.3.3) . . . . . . . 1-6

1.2.2.4 Neutron Interferometer (WBS 1.4.2.8.1) . . . . . . . . . . . . . 1-7

1.2.3 System Configuration and Essential Features . . . . . . . . . . . . 1-7

1.2.3.1 Thermal Nuclear Physics (WBS 1.4.3.1) . . . . . . . . . . . 1-7

1.2.3.1.1 Through tube loading station (WBS 1.4.3.1.1) . . . . . . 1-7

1.2.3.1.2 Ultrahigh resolution $\gamma$-spectroscopy (WBS 1.4.3.1.2) $\ldots \ldots \ldots$ 1-7

1.2.3.2 Polarized Nuclear Physics (WBS 1.4.3.2) $\ldots \ldots \ldots \ldots \ldots \ldots \ldots \ldots$. . . . . . . . .

1.2.3.2.1 Precision $\gamma$-ray bolometer (WBS 1.4.3.2.1) $\ldots \ldots \ldots \ldots \ldots$ 1-7

1.2.3.2.2 Weak interaction physics (WBS 1.4.3.2.2) . . . . . . 1-11

1.2.3.2.3 Parity violation (WBS 1.4 .3 .2 .3$) \ldots \ldots \ldots \ldots \ldots \ldots$ 1-11

1.2.3.3 General Nuclear Physics (WBS 1.4.3.3) . . . . . . . . . . . . 1-11

1.2.3.3.1 Nuclear structure (WBS 1.4.3.3.1) . . . . . . . . 1-11

1.2.3.3.2 Nuclear spectroscopy (WBS 1.4.3.3.2) . . . . . . . . 1-11

1.2.3.3.3 Isotope separation on-line (WBS 1.4.3.3.3) . . . . . . 1-11

1.2.3.4 Neutron Interferometer (WBS 1.4.2.8.1) . . . . . . . . . . . 1-12

1.2.3.4.1 Neutron monochromator . . . . . . . . . . . 1-12

1.2.3.4.2 Interferometer enclosure . . . . . . . . . . . . 1-12

1.2.4 Maintenance . . . . . . . . . . . . . .

1.2.4.1 Scheduled Maintenance . . . . . . . . . . . . . . . . . 1-12

1.2.4.2 Unscheduled Maintenance . . . . . . . . . . . . . . . 1-12

1.2.4.3 Sample Preparation Area . . . . . . . . . . . . . . . . . 1-12

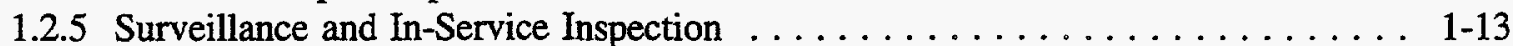

1.2.5.1 Thermal Nuclear Physics (WBS 1.4.3.1) . . . . . . . . . . . . . 1-13

1.2.5.1.1 Through tube loading station (WBS 1.4.3.1.1) . . . . . 1-13

1.2.5.1.2 Ultrahigh resolution $\gamma$-spectroscopy (WBS 1.4.3.1.2) $\ldots \ldots$ 1-13

1.2.5.2 Polarized Nuclear Physics (WBS 1.4.3.2) . . . . . . . . . . . 1 1-13

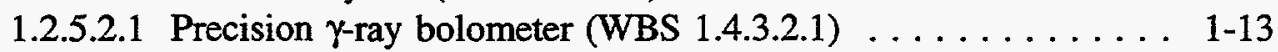

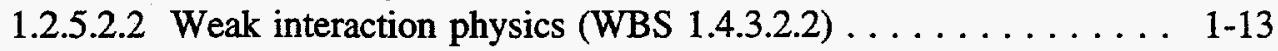

1.2.5.2.3 Parity violation (WBS 1.4 .3 .2 .3$) \ldots \ldots \ldots \ldots \ldots 1-13$

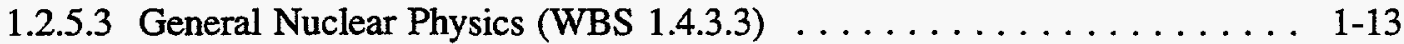

1.2.5.3.1 Nuclear spectroscopy (WBS 1.4.3.3.1) . . . . . . . . . . 1-13

1.2.5.3.2 Nuclear structure (WBS 1.4.3.3.2) . . . . . . . . . . . . . 1-14

1.2.5.3.3 Isotope separation on-line (WBS 1.4.3.3.3) . . . . . . . 1-14

1.2.5.4 Neutron Interferometer (WBS 1.4.2.8.1) . . . . . . . . . . . . . . 1-14

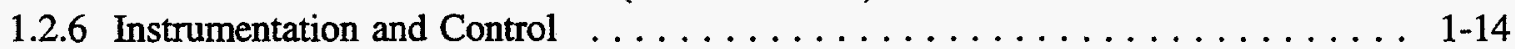

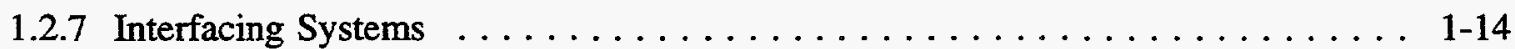

1.2.7.1 Electrical ........................ 1-14

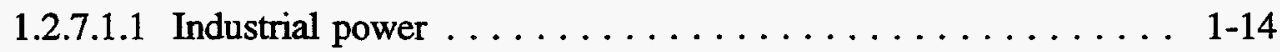

1.2.7.1.2 Clean power . . . . . . . . . . . . . . . 1-14

1.2.7.1.3 Uninterruptible power . . . . . . . . . . . . 1-14

1.2.7.2 Compressed Gas . . . . . . . . . . . . . . . . . . 15

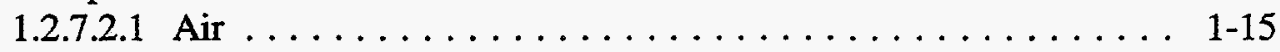

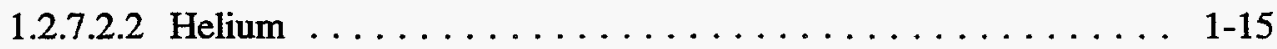

1.2.7.2.3 Nitrogen . . . . . . . . . . . . . . 1-15 


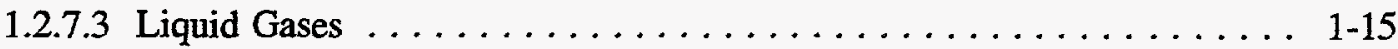

1.2.7.3.1 Helium ............................ 1-15

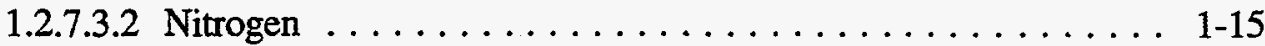

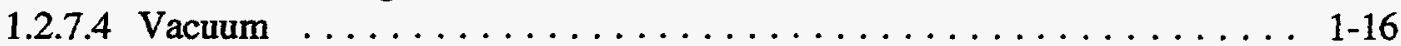

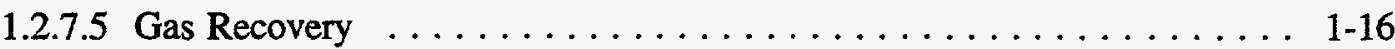

1.2.7.5.1 Vacuum pump exhaust $\ldots \ldots \ldots \ldots \ldots \ldots \ldots \ldots \ldots \ldots$

1.2.7.5.2 Helium . . . . . . . . . . . . . . . . . . . . . 16

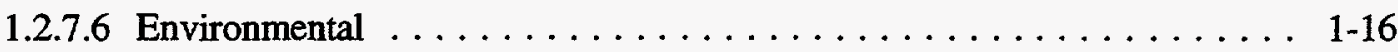

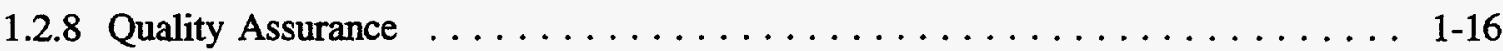

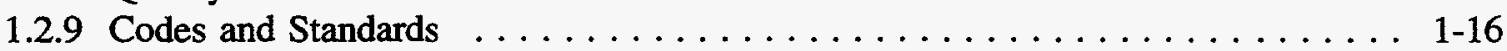

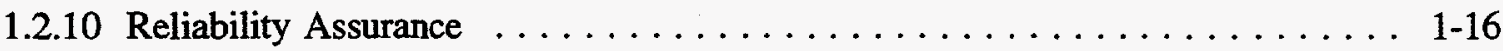

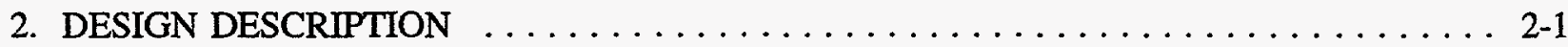

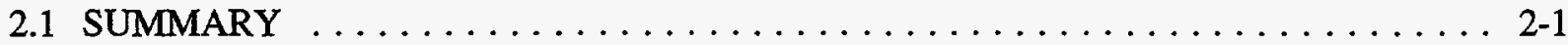

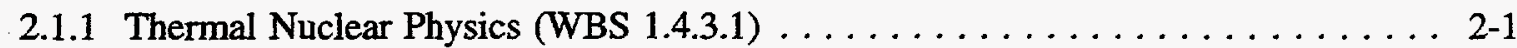

2.1.1.1 Through Tube Loading Station (WBS 1.4.3.1.1) ........... 2-1

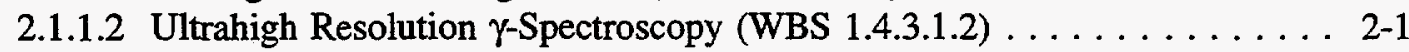

2.1.2 Polarized Nuclear Physics (WBS 1.4.3.2) $\ldots \ldots \ldots \ldots \ldots \ldots \ldots \ldots \ldots . \ldots \ldots . .1$

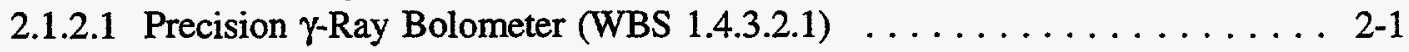

2.1.2.2 Weak Interaction Physics (WBS 1.4.3.2.2) $\ldots \ldots \ldots \ldots \ldots \ldots \ldots .2-1$

2.1.2.3 Parity Violation (WBS 1.4 .3 .2 .3$) \ldots \ldots \ldots \ldots \ldots \ldots \ldots \ldots \ldots \ldots \ldots \ldots .2-2$

2.1.3 General Nuclear Physics (WBS 1.4.3.3) $\ldots \ldots \ldots \ldots \ldots \ldots \ldots \ldots \ldots \ldots .2-2$

2.1.3.1 Nuclear Structure (WBS 1.4.3.3.1) . . . . . . . . . . . . 2-2

2.1.3.2 Nuclear Spectroscopy (WBS 1.4 .3 .3 .2$) \ldots \ldots \ldots \ldots \ldots \ldots \ldots .2-2$

2.1.3.3 Isotope Separation On-Line (WBS 1.4.3.2.3) $\ldots \ldots \ldots \ldots \ldots \ldots \ldots .2-2$

2.1.4 Neutron Interferometer (WBS 1.4.2.8.1) $\ldots \ldots \ldots \ldots \ldots \ldots \ldots \ldots .2-2$

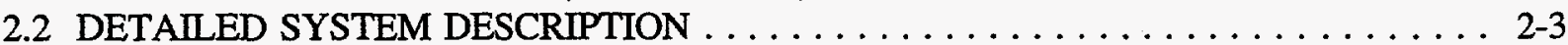

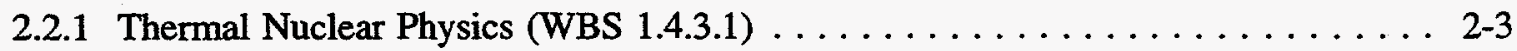

2.2.1.1 Through Tube Loading Station (WBS 1.4.3.1.1) .......... 2-3

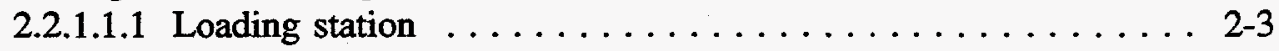

2.2.1.1.2 Sample irradiation assembly $\ldots \ldots \ldots \ldots \ldots \ldots \ldots \ldots, 2-3$

2.2.1.1.3 Through Tube .................... 2-3

2.2.1.2 Ultrahigh Resolution $\gamma$-Ray Spectroscopy (WBS 1.4.3.1.2) $\ldots \ldots \ldots \ldots$ 2-4

2.2.1.2.1 Crystal $\gamma$-ray spectrometer $\ldots \ldots \ldots \ldots \ldots \ldots \ldots \ldots, 2-4$

2.2.1.2.2 Pair formation $\gamma$-ray spectrometer $\ldots \ldots \ldots \ldots \ldots \ldots \ldots 2-5$

2.2.2 Polarized Nuclear Physics (WBS 1.4.3.2) $\ldots \ldots \ldots \ldots \ldots \ldots \ldots \ldots \ldots .2-5$

2.2.2.1 Precision $\gamma$-Ray Bolometer (WBS 1.4.3.2.1) $\ldots \ldots \ldots \ldots \ldots \ldots .2-5$

2.2.2.2 Weak Interaction Physics (WBS 1.4.3.2.2) $\ldots \ldots \ldots \ldots \ldots \ldots . . .2-5$

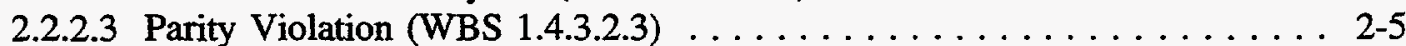

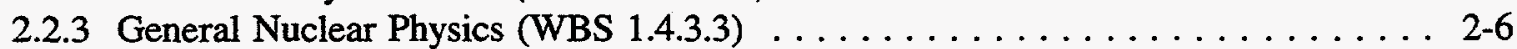

2.2.3.1 Nuclear Structure (WBS 1.4.3.3.1) . . . . . . . . . . . . .

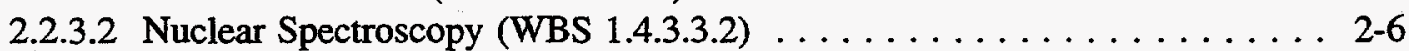

2.2.3.3 Isotope Separation On-Line (WBS 1.4.3.3.3) $\ldots \ldots \ldots \ldots \ldots \ldots \ldots .2-6$

2.2.3.3.1 Production of fission products $\ldots \ldots \ldots \ldots \ldots \ldots \ldots \ldots \ldots \ldots$

2.2.3.3.2 Separation of fission products $\ldots \ldots \ldots \ldots \ldots \ldots \ldots \ldots, 2-6$

2.2.3.3.3 Measurement station .................... 2-6

2.2.4 Neutron Interferometer (WBS 1.4.2.8.1) $\ldots \ldots \ldots \ldots \ldots \ldots \ldots \ldots \ldots .2-7$

2.2.4.1 Double-Crystal Monochromator .................. 2-7 
2.2.4.2 Neutron Interferometer $\ldots \ldots \ldots \ldots \ldots \ldots \ldots \ldots$. . . . . . . . . . . .

2.3 SYSTEM PERFORMANCE CHARACTERISTICS $\ldots \ldots \ldots \ldots \ldots \ldots \ldots \ldots \ldots \ldots$

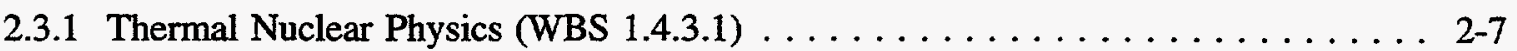

2.3.1.1 Through Tube Loading Station (WBS 1.4.3.1.1) . . . . . . . . . 2-7

2.3.1.2 Ultrahigh Resolution $\gamma$-Spectroscopy (WBS 1.4.3.1.2) . . . . . . . . 2-8

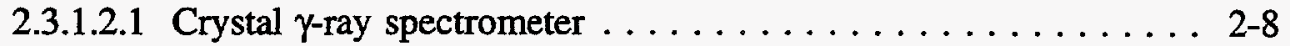

2.3.1.2.2 Pair formation $\gamma$-spectrometer . . . . . . . . . . . 2-9

2.3.2 Polarized Nuclear Physics (WBS 1.4.3.2) . . . . . . . . . . . . . . . . . . 2-9

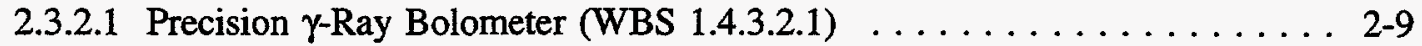

2.3.2.2 Weak Interaction Physics (WBS 1.4.3.2.2) $\ldots \ldots \ldots \ldots \ldots \ldots \ldots$. . . . .

2.3.2.3 Parity Violation (WBS 1.4 .3 .2 .3 ) . . . . . . . . . . . . . . 2-9

2.3.3 General Nuclear Physics (WBS 1.4.3.3) . . . . . . . . . . . . . . . . 2-9

2.3.3.1 Nuclear Structure (WBS 1.4.3.3.1) . . . . . . . . . . . 2-9

2.3.3.2 Nuclear Spectroscopy (WBS 1.4.3.3.2) . . . . . . . . . . 2-10

2.3.3.3 Isotope Separation On-Line (WBS 1.4.3.3.3) . . . . . . . . . 2-10

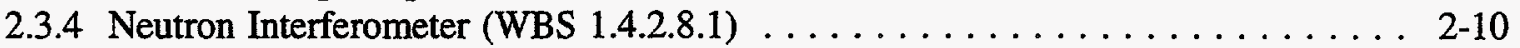

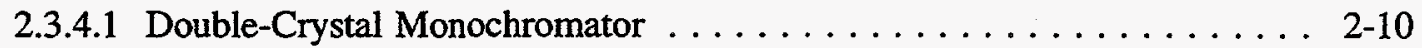

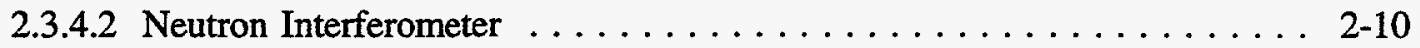

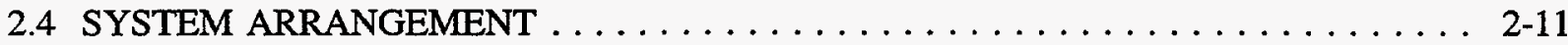

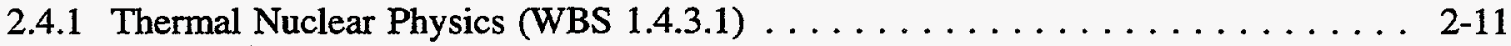

2.4.1.1 Through Tube Loading Station (WBS 1.4.3.1.1) . . . . . . . . . 2-11

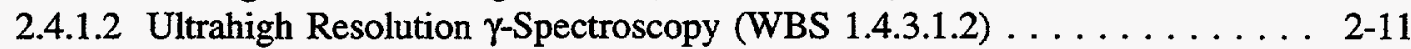

2.4.1.2.1 Crystal spectrometer . . . . . . . . . . . . . . . 2-11

2.4.1.2.2 Pair formation spectrometer . . . . . . . . . . . 2-14

2.4.2 Polarized Nuclear Physics (WBS 1.4.3.2) . . . . . . . . . . . . . 2-14

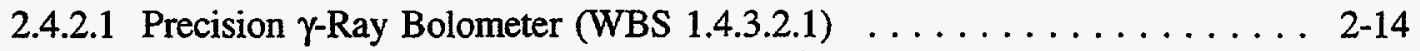

2.4.2.2 Weak Interaction Physics (WBS 1.4.3.2.2) $\ldots \ldots \ldots \ldots \ldots \ldots \ldots$ 2-14

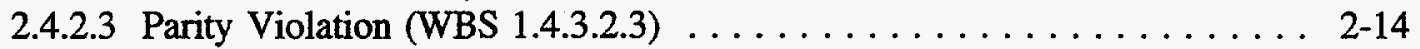

2.4.3 General Nuclear Physics (WBS 1.4.3.3) . . . . . . . . . . . . . 2 2-14

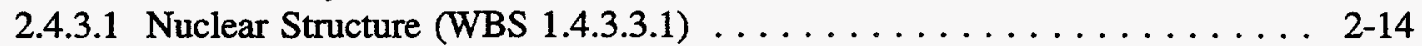

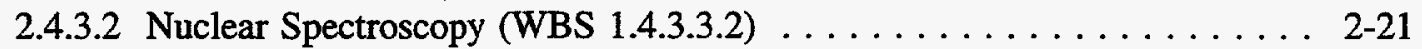

2.4.3.3 Isotope Separation On-Line (WBS 1.4.3.3.3) . . . . . . . . . . 2-21

2.4.3.3.1 Ion source and beam transport . . . . . . . . . 2-21

2.4.3.3.2 Isotope separation $\ldots \ldots \ldots \ldots \ldots \ldots \ldots \ldots . \ldots \ldots$ 2-21

2.4.3.3.3 Measurement station . . . . . . . . . . . . . 2-21

2.4.4 Neutron Interferometer (WBS 1.4.2.8.1) . . . . . . . . . . . . 2-21

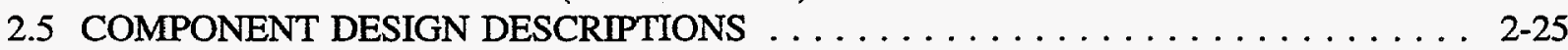

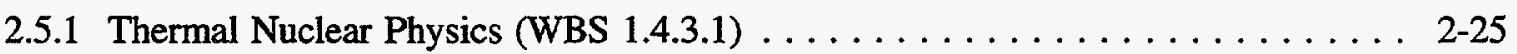

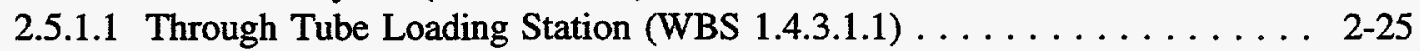

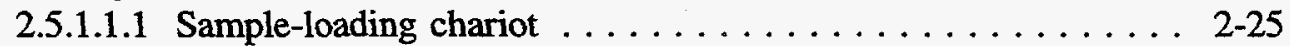

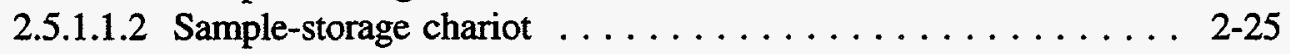

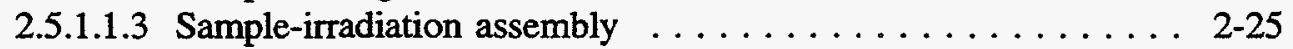

2.5.1.1.4 Work area . . . . . . . . . . . . . . . 2-26

2.5.1.2 Ultrahigh Resolution $\boldsymbol{\gamma}$-Ray Spectroscopy (WBS 1.4.3.1.2) . . . . . . 2-26

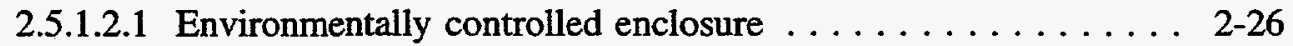

2.5.1.2.2 Crystal spectrometer . . . . . . . . . . . . 2-26

2.5.1.2.3 Pair formation spectrometer . . . . . . . . . . 2-27 


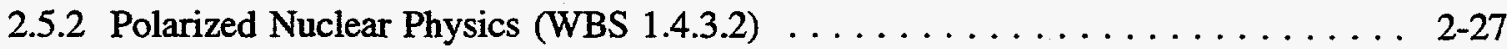

2.5.2.1 Precision $\gamma$-Ray Bolometer (WBS 1.4.3.2.1) $\ldots \ldots \ldots \ldots \ldots \ldots$ 2-27

2.5.2.2 Weak Interaction Physics (WBS 1.4.3.2.2) $\ldots \ldots \ldots \ldots \ldots \ldots \ldots$. $\ldots \ldots$

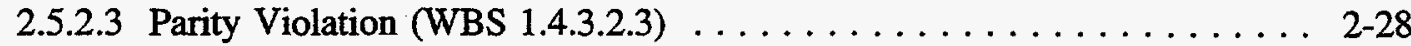

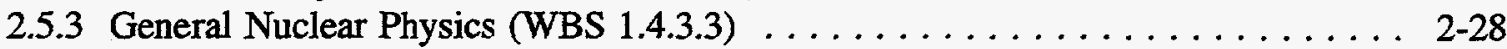

2.5.3.1 General Detector Pool . . . . . . . . . . . . . . . . 2-28

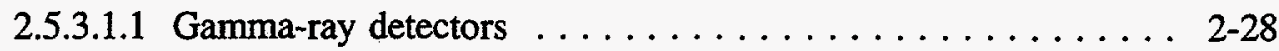

2.5.3.1.2 Particle detectors ................... 2-29

2.5.1.2.2 Charged-particle detectors . . . . . . . . . . . . 2-29

2.5.1.2.3 Neutron detectors . . . . . . . . . . . . . . . . 2-29

2.5.3.2 Nuclear Structure (WBS 1.4.3.3.1) . . . . . . . . . . . . 2-30

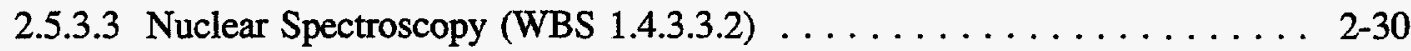

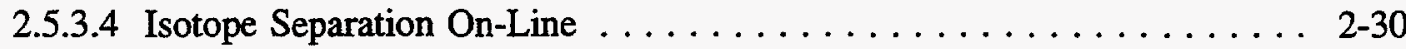

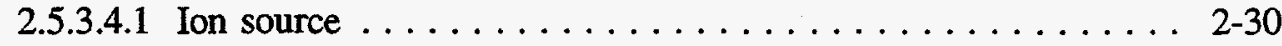

2.5.3.4.2 Isotope separation $\ldots \ldots \ldots \ldots \ldots \ldots \ldots \ldots \ldots \ldots \ldots \ldots \ldots$ 2-31

2.5.3.4.3 Measurement station . . . . . . . . . . . . . . 2-31

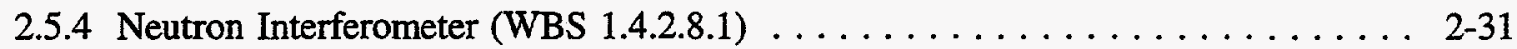

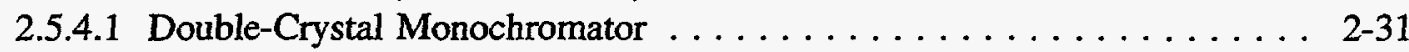

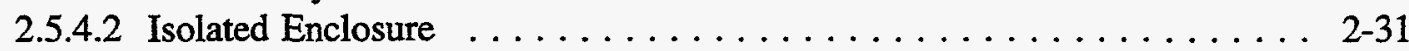

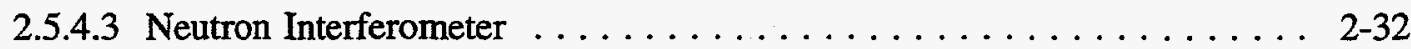

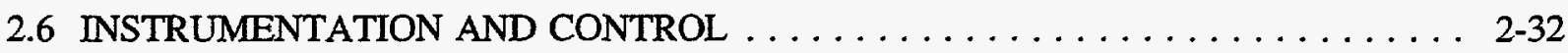

2.6.1 Thermal Nuclear Physics (WBS 1.4.3.1) . . . . . . . . . . . . 2-32

2.6.1.1 Through Tube Loading Station (WBS 1.4.3.1.1) . . . . . . . . 2-32

2.6.1.2 Ultrahigh Resolution $\gamma$ Spectroscopy (WBS 1.4.3.1.2) . . . . . . . 2-33

2.6.2 Polarized Nuclear Physics (WBS 1.4.3.2) . . . . . . . . . . . . . . 2-33

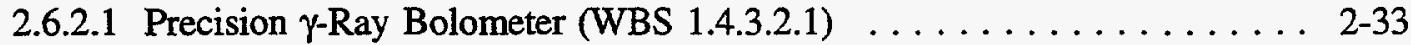

2.6.2.2 Weak Interaction Physics (WBS 1.4.3.2.2) $\ldots \ldots \ldots \ldots \ldots \ldots$. $\ldots \ldots$

2.6.2.3 Parity Violation (WBS 1.4 .3 .2 .3$) \ldots \ldots \ldots \ldots \ldots \ldots \ldots . \ldots \ldots \ldots$

2.6.2 General Nuclear Physics (WBS 1.4.3.3) . . . . . . . . . . . . . 2-33

2.6.3.1 Nuclear Structure (WBS 1.4.3.3.1) . . . . . . . . . . . . 2-33

2.6.3.2 Nuclear Spectroscopy (WBS 1.4.3.3.2) . . . . . . . . . . 2-33

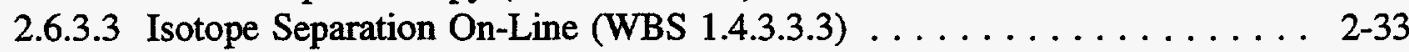

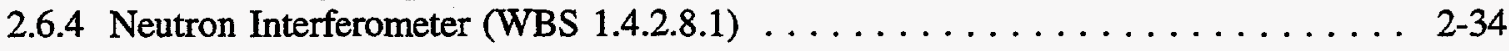

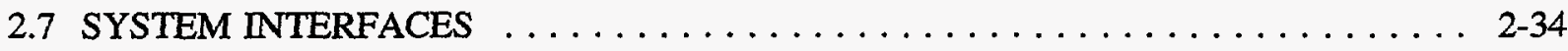

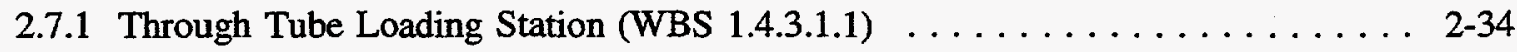

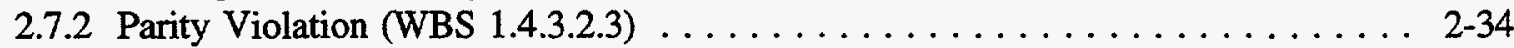

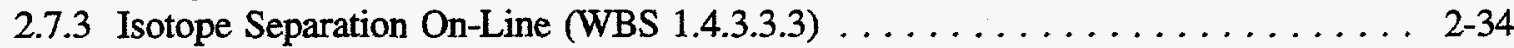

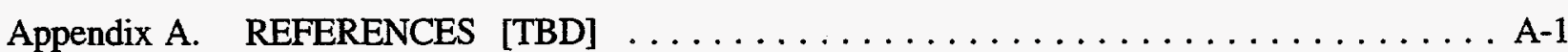

Appendix B. PARAMETERS LIST $[\mathrm{TBD}] \ldots \ldots \ldots \ldots \ldots \ldots \ldots \ldots \ldots \ldots \ldots \ldots \ldots$ B-1

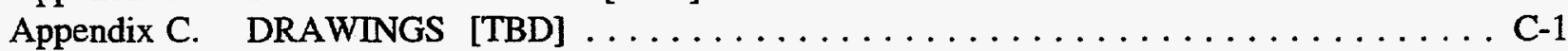

Appendix D. INSTRUMENT LIST $[$ TBD] $\ldots \ldots \ldots \ldots \ldots \ldots \ldots \ldots \ldots \ldots \ldots \ldots \ldots$ D-1

Appendix E. VALVE LIST $[\mathrm{TBD}] \ldots \ldots \ldots \ldots \ldots \ldots \ldots \ldots \ldots \ldots \ldots \ldots \ldots \ldots \ldots \ldots$

Appendix F. EQUIPMENT LIST $[\mathrm{TBD}] \ldots \ldots \ldots \ldots \ldots \ldots \ldots \ldots \ldots \ldots \ldots \ldots \ldots \ldots$

Appendix G. LINE LIST $[$ TBD] $\ldots \ldots \ldots \ldots \ldots \ldots \ldots \ldots \ldots \ldots \ldots \ldots \ldots \ldots \ldots \ldots$

Appendix $H . \quad$ PRIMARY SYSTEM INTERFACES $[\mathrm{TBD}] \ldots \ldots \ldots \ldots \ldots \ldots \ldots \ldots \ldots \ldots$

Appendix I. SECONDARY SYSTEM INTERFACES [TBD] $\ldots \ldots \ldots \ldots \ldots \ldots \ldots \ldots \ldots$

Appendix J. APPLICABLE STANDARDS, CODES AND GUIDES $\ldots \ldots \ldots \ldots \ldots \ldots \ldots . . .$. 
Appendix K. SYSTEM/COMPONENT SAFETY CLASSIFICATION AND STRUCTURAL DESIGN BASIS $\mathrm{K}-1$

Appendix L. ELECTRICAL LOAD LIST [TBD] $\ldots \ldots \ldots \ldots \ldots \ldots \ldots \ldots \ldots \ldots \ldots \ldots \ldots$ Appendix M. REQUIREMENTS TRACEABILITY INDEX [TBD] .............. M-1 Appendix N. CONFORMANCE WITH REGULATORY (NRC, DOE)

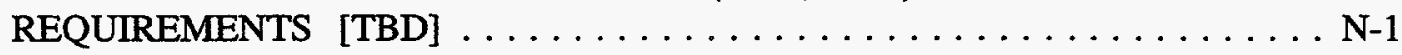

Appendix O. HOLD/TBD RESPONSIBILITY-STATUS LIST [TBD] $\ldots \ldots \ldots \ldots \ldots \ldots$ O-1 Appendix P. CONTRAINMENT PENETRATION SCHEDULE $[\mathrm{TBD}] \ldots \ldots \ldots \ldots \ldots$ P-1 


\section{LIST OF FIGURES}

$\underline{\text { Figure }}$

$\underline{\text { Page }}$

1.1. Plan view for the ground floor beam room $\ldots \ldots \ldots \ldots \ldots \ldots \ldots \ldots \ldots$

1.2. Plan view for the second floor beam room $\ldots \ldots \ldots \ldots \ldots \ldots \ldots \ldots \ldots$

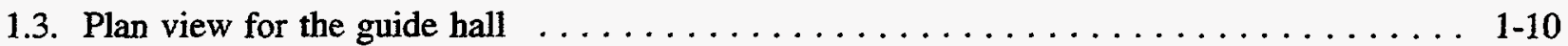

2.1. Schematic diagram of through tube loading station operations $\ldots \ldots \ldots \ldots \ldots \ldots \ldots$ 2-12

2.2. Possible geometries of the crystal gamma-ray spectrometer $\ldots \ldots \ldots \ldots \ldots \ldots \ldots \ldots$

2.3. Schematic plan view of the ultrahigh resolution spectroscopy station when configured for the formation spectrometer $\ldots \ldots \ldots \ldots \ldots \ldots \ldots \ldots$

2.4. Schematic side view of sample area in the helium dilution

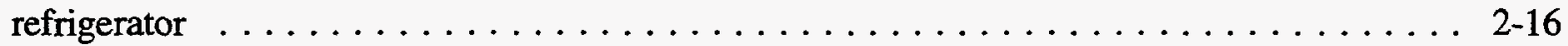

2.5. Schematic view of the helium dilution refrigerator $\ldots \ldots \ldots \ldots \ldots \ldots \ldots \ldots \ldots \ldots$

2.6. Schematic plan view of the "weak interaction" physics and

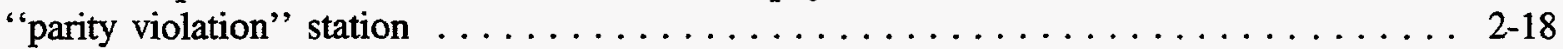

2.7. Schematic plan view of sample area for nuclear structure and nuclear spectroscopy stations . . . . . . . . . . . . . . . . . . . . 2-19

2.8. Schematic horizontal section through flexible detector array $\ldots \ldots \ldots \ldots \ldots \ldots \ldots$

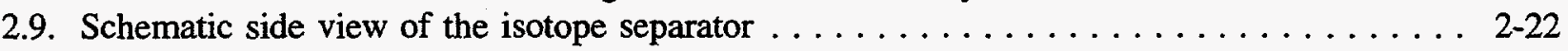

2.10. Schematic plan view of the isotope separator $\ldots \ldots \ldots \ldots \ldots \ldots \ldots \ldots \ldots \ldots \ldots \ldots$

2.11. Schematic of the $L-7$ neutron interferometer $\ldots \ldots \ldots \ldots \ldots \ldots \ldots \ldots \ldots \ldots \ldots . \ldots \ldots$ 



\section{LIST OF TABLES}

Table

$\underline{\text { Page }}$

There are no tables in this SDD. 


\section{ACRONYMS}

$\mathrm{A} / \mathrm{E} \quad$ architect/engineer

ALARA as low as reasonably achievable

ANS Advanced Neutron Source

BGO bismuth germanate

DOE Department of Energy

HPGe hyperpure germanium

IIL Institut Laue-Langevin

ISDD Integrating System Design Description

ISOL Isotope Separation On-Line

PDR Plant Design Requirements

PGAA prompt gamma activation analysis

RIB radioactive ion beam

SANS Small Angle Neutron Scattering

SDD System Design Description

TBD to be determined

WBS work breakdown structure 

The nuclear and fundamental physics instruments are listed under work breakdown structure (WBS) element 1.4.3. This element covers instruments that are situated on neutron beams or guides but that differ qualitatively from the instruments classified under neutron scattering, some of which (e.g., certain optical and ultracold neutron instruments) also could be considered fundamental physics instruments from a scientific viewpoint. The instruments are situated in the reactor and guide-hall buildings (SDD-52 and SDD-54).

Instruments covered in this System Design Description (SDD) fall into two basically different categories. The first type includes essentially "roll-in, roll-out" instruments that need a beam or guide station, a beam preparation system, a safety system, and standard services. The project thus provides all essential interfaces to the instrument, but in general the design and construction of these particular instruments lies outside the scope of the currently proposed instrument complement for the project. The beam preparation system includes the monochromator, polarizer, and collimators that define the beam and associated biological shielding that must reduce the level of radiation at its external surface to below $25 \mu \mathrm{Sv}(2.5 \mathrm{mrem} / \mathrm{h})$. The instrument safety system defines the distance of safest approach and provides access control and interlock monitoring to ensure operator safety.

The second type includes instruments that require specialized installations and may necessitate special safety systems in addition to those normally provided at a beam station, either because of a need to handle small amounts of radioactive material or because of an intrinsic danger associated with the experiment, such as handling liquid hydrogen. These instruments may require specific interfaces to building structures. The extent of specialized interfaces provided by the project for these instruments is being developed separately for each case. At least the minimum interface to permit future installation of any given instrument will be provided. 


\section{FUNCTIONS AND DESIGN REQUIREMENT}

\subsection{FUNCTION}

\subsubsection{Thermal Nuclear Physics (WBS 1.4.3.1)}

The through beam tube (WBS 1.4.1.2.2) functions as a means to introduce nuclei that undergo $(n, \gamma)$ reactions into the high-flux region of the reflector and to allow the $\gamma$ rays to be viewed without the spectral contamination that would arise from background generated by the end window of a normal thermal beam tube.

\subsubsection{Through Tube Loading Station (WBS 1.4.3.1.1)}

The through tube loading station receives samples and permits their insertion into and recovery from the beam tube. Transfer to a shielded handling cask is provided for samples that become activated in the high flux. The loading station terminates the tube and provides the required radiation shielding.

\subsubsection{Ultrahigh Resolution $\gamma$-Spectrometer (WBS 1.4.3.1.2)}

The function of this spectrometer is to measure $\gamma$-ray energies with very high precision so that definitive nuclear levels in the emitting nucleus can be found by means of the Ritz recombination principle. In addition, the observed Doppler broadening induced by prior gamma transitions can be used to measure the lifetimes of intermediate nuclear states.

\subsubsection{Polarized Nuclear Physics (WBS 1.4.3.2)}

The general function of the polarized nuclear physics stations is to provide work areas served by intense, cold polarized beams for nuclear physics experiments.

\subsubsection{Precision $\gamma$-ray Bolometer (WBS 1.4.3.2.1)}

This instrument will function as a development station for precision $\gamma$-ray spectroscopy by means of a bolometer detector. This is a novel technique that measures photons released when $\gamma$ rays interact with material; it functions essentially as a low-temperature $(5-\mathrm{mK})$ calorimeter. It will also make possible the study of neutron capture by polarized nuclei.

\subsubsection{Weak Interaction Physics (WBS 1.4.3.2.2)}

This station provides a polarized beam and a work area suitable for the study of weak interactions. Applications include the measurement of the nuclear optical rotation of the beam as it passes through matter and the "in-beam" measurement of the properties of the neutron itself. 


\subsubsection{Parity Violation (WBS 1.4.3.2.3)}

The function of this station is to provide an intense polarized cold neutron beam and a location suitable for safely performing experiments involving multiliter quantities of liquid hydrogen. The beam is used to investigate weak interactions by creating polarized, excited nuclei by the capture of spinpolarized neutrons and studying the asymmetry of the radiation emitted by the excited nucleus.

\subsubsection{General Nuclear Physics (WBS 1.4.3.3)}

\subsubsection{Nuclear Structure (WBS 1.4.3.3.1)}

This functions as a general purpose cold neutron workstation for recording the energy and intensity of $\gamma$ radiation, electrons ( $\beta$ rays and internal conversion) and charged particles following neutron capture.

\subsubsection{Nuclear Spectroscopy (WBS 1.4.3.3.2)}

This functions as a general purpose cold neutron workstation for recording the energy and intensity of prompt $\gamma$ rays following neutron capture.

\subsubsection{Isotope Separation On-Line (WBS 1.4.3.3.3)}

The functions of the isotope separation on-line (ISOL) facility are:

- to introduce small (mg) quantities of fissile isotopes into a region of high thermal flux, thus producing neutron-rich fission-product nuclides far from the line of beta stability,

- to provide a means of extracting these nuclides and separating them,

- to deliver these nuclides to a counting station so that their nuclear properties can be studied,

- to provide a source of unusual radioactive ions for ion implantation studies of electron emission, channelling, and hyperfine interactions associated with implanted radioactive atoms, and to be accessible such that the facility could be used as an ion source to be coupled to a heavy ion accelerator for the generation of neutron-rich radioactive heavy ion beams.

\subsubsection{Neutron Interferometer (WBS 1.4.2.8.1)}

This instrument is also described in SDD-42, but because its dual-purpose usage will be in the field of fundamental physics, it is also included in this document (SDD-43).

The neutron interferometer uses a beam of monochromatic neutrons, produced by a double crystal system, to measure interference effects in neutron beams diffracted by high-purity crystals. Such interference patterns can be used to determine better the fundamental interactions of the neutron itself and to determine standard neutron scattering amplitudes, which are of use in general neutron scattering work. 


\subsection{DESIGN REQUIREMENTS}

\subsubsection{Operational Requirements}

General design requirements, particularly for associated work areas and interfacing services, are the same as for neutron scattering instruments (SDD-42).

\subsubsection{Thermal Nuclear Physics (WBS 1.4.3.1)}

A cylindrical beam tube of $200 \mathrm{~mm}$ (7.9 in.) diam is required. It should pass through the reflector vessel.

\subsection{Through tube loading station (WBS 1.4.3.1.1)}

The through tube loading station must

provide a complete beam stop and biological shield at one end of the through beam tabe; provide a means of loading/unloading small samples of stable isotopes into the shielding; provide a means of loading/unloading several samples independently; provide a safe means of moving samples from the loading station into the center of the beam tube; provide a means for storing irradiated samples for later reuse; provide for monitoring of the sample integrity during sample loading, unloading, and irradiation; provide a means for the safe disposal of highly active irradiated samples; and provide for operation under inert gas atmospheres with direct coupling to the through tube itself.

\subsection{Ultrahigh resolution $\gamma$-spectroscopy (WBS 1.4.3.1.2)}

This station must be situated at the end of the through beam tube opposite the end where the loading station is situated. It must interface to a crystal $\gamma$-ray spectrometer of variable geometry for the measurement of energies up to a few million electron volts and for the measurement of Doppler broadened gamma transitions. Both the $\gamma$ and neutron backgrounds must be low, especially for the latter application. For measuring energies greater than $1.5 \mathrm{MeV}$ with higher sensitivity, the station should also contain a pair-formation $\gamma$ ray spectrometer.

\subsubsection{Polarized Nuclear Physics (WBS 1.4.3.2)}

\subsection{Precision $\gamma$-ray bolometer (WBS 1.4.3.2.1)}

This requires a focussed white beam of cold neutrons. There must be an option to polarize the beam if desired, either by the focussing device or by an insertion polarizer, and an optional filter. The cryogenic device used to cool the samples to operating temperature must have an access port to allow neutrons to impinge on the sample. A separate loading facility to allow the rapid exchange of samples without dismantling the station is also required. 


\subsection{Weak interaction physics (WBS 1.4.3.2.2)}

This requires a focussed white beam of cold neutrons. There must be an option to polarize the beam if desired, either by the focussing device or by an insertion polarizer, and an optional filter.

\subsection{Parity violation (WBS 1.4.3.2.3)}

This requires a polarized, clean beam of cold neutrons. Optional beam focussing should be provided. The beam must be delivered to a workspace designed for the safe handling of experiment targets containing up to $20 \mathrm{~L}$ (5.3 gal) of liquid hydrogen and designed to withstand the worst-case accident involving the target.

\subsubsection{General Nuclear Physics (WBS 1.4.3.3)}

\subsection{Nuclear structure (WBS 1.4.3.3.1)}

This requires a beam of cold neutrons. Optional beam filtering and focussing should be provided. The beam should be delivered to a workspace allowing for the use of symmetric arrangements of gamma detectors. Gamma background should be as low as is reasonably achievable (ALARA).

\subsection{Nuclear spectroscopy (WBS 1.4.3.3.2)}

This requires a beam of cold neutrons. Optional beam filtering, polarization and focussing should be provided. The beam should be delivered to a workspace allowing for symmetric arrangements of gamma detectors to be used. Gamma background should be minimized.

\subsection{Isotope separation on-line (WBS 1.4.3.3.3)}

The ISOL facility requirements are

- a slant thermal beam tube of $200 \mathrm{~mm}$ (7.87 in.) diam (see SDD-41),

- an ion source situated in the neutron beam and located in a small hot cell constructed as an integral part of the reactor biological shielding,

. a mass separator located external to the hot cell but requiring biological shielding,

- ion transport lines that may penetrate containment to carry ion beams to a remote experimental area in a future expansion, and

- monitoring of safe operation during loading, unloading, and irradiation of the target.

\subsubsection{Neutron Interferometer (WBS 1.4.2.8.1)}

The monochromator system must be capable of delivering a beam of monochromatic neutrons with variable wavelength in the range from 0.13 to $0.55 \mathrm{~nm}(1.3$ to $5.5 \AA)$. This beam will be delivered to the neutron interferometer station. Precision control is necessary to ensure that the point of delivery of the monochomatized beam to the interferometer is independent of the neutron energy. The interferometer position itself must provide the best possible thermal, vibrational, acoustic, and seismic isolation. 


\subsubsection{Structural Requirements}

\subsubsection{Thermal Nuclear Physics (WBS 1.4.3.1)}

\subsection{Through tube loading station (WBS 1.4.3.1.1)}

The through tube itself should be of such material and design that it is capable of maintaining the integrity of an atmosphere of inert gas at pressures ranging from $500 \mathrm{~Pa}(0.072 \mathrm{psi})$ to $0.2 \mathrm{MPa}(29.0$ psi). The closure of the tube after introduction of a sample should maintain the integrity of this atmosphere. The end window of the through tube on the side of the ultrahigh resolution $\gamma$-spectroscopy station (WBS 1.4.3.1.2) should be thin enough to minimize the absorption of the $\gamma$ rays at energies as low as $30 \mathrm{keV}$ while maintaining the integrity of the internal atmosphere.

Floor loading capabilities in the area of the through tube loading station must be compatible with the extensive biological shielding required.

\subsection{Ultrahigh resolution $\gamma$-spectroscopy (WBS 1.4.3.1.2)}

The crystal $\gamma$-ray spectrometer must be housed in its own blockhouse within which the atmospheric environment can be closely controlled and monitored. A floor space of $5 \mathrm{~m}$ (16.4 ft) perpendicular to the beam axis is required and this should be asymmetric with respect to this axis, having a $1.5 \mathrm{~m}: 3.5 \mathrm{~m}(4.9 \mathrm{ft}: 11.5 \mathrm{ft})$ split. A total length of $7 \mathrm{~m}(22.96 \mathrm{ft})$ along the beam axis direction is required.

Vibration should be minimized by standing the sensitive section of the spectrometer on a floor that is decoupled, as far as possible, from the rest of the reactor building. Acoustic insulation is also necessary.

\subsubsection{Polarized Nuclear Physics (WBS 1.4.3.2)}

\subsection{Precision $\gamma$-ray bolometer (WBS 1.4.3.2.1)}

The only device capable of cooling samples to the required temperature $5 \mathrm{mK}$ is a helium dilution refrigerator. Such a device has a number of structural requirements.

- The bolometer itself will be sensitive to excessive vibrations and so the whole device should be mounted on a section of floor which is decoupled, as far as possible, from the surrounding area. To enable sample loading, a rigid platform is required at the top of the device $\sim 4 \mathrm{~m}(13.1 \mathrm{ft})$, capable of supporting several people and dewars of liquefied gasses.

The actual sample loading procedure involves the vertical introduction of a long syphon vertically into the device. Thus, a clearance of at least a further $4 \mathrm{~m}(13.1 \mathrm{ft})$ above the loading platform is required.

A total floor area of $4 \mathrm{~m}$ by $4 \mathrm{~m}(13.1 \mathrm{ft}$ by $13.1 \mathrm{ft})$ positioned symmetrically with respect to the beam axis is required. 
A floor space of $6 \mathrm{~m}(19.7 \mathrm{ft})$ [ $3 \mathrm{~m}(9.8 \mathrm{ft})$ either side of the beam axis] by $8 \mathrm{~m}(26.2 \mathrm{ft})$ is required. Access must be possible for lifting equipment to insert and remove large, user-supplied equipment. The area should be shielded to reduce both neutron and $\gamma$-ray background. A small, separate blockhouse for the optional insertion of neutron polarizers or filters should be located in front of the experimental area. This area should have a floor space of approximately $2 \mathrm{~m}$ by $2 \mathrm{~m}(6.6 \mathrm{ft}$ by $6.6 \mathrm{ft})$.

\subsection{Parity violation (WBS 1.4.3.2.3)}

This experimental station should be completely enclosed such that a worst-case accident involving multiliter quantities of liquid hydrogen will not compromise the safety of the rest of the building. To this end it is proposed to surround the area with rigid walls but provide only a thin roof to direct any blast upward and out of the building.

Access must be possible for lifting equipment to insert and remove large, user-supplied equipment. The area should be shielded to reduce both neutron and $\gamma$-ray background. A small, separate, blockhouse for the optional insertion of neutron polarizers or filters should be located in front of the experimental area. This area should have a floor space of approximately $2 \mathrm{~m}$ by $2 \mathrm{~m}(6.6 \mathrm{ft}$ by $6.6 \mathrm{ft}$ ). The experimental area itself must provide symmetric access to the beam.

\subsubsection{General Nuclear Physics (WBS 1.4.3.3)}

\subsection{Nuclear structure (WBS 1.4.3.3.1)}

An experimental area of approximately $3 \mathrm{~m}$ by $3 \mathrm{~m}(9.8 \mathrm{ft}$ by $9.8 \mathrm{ft})$, symmetric to the beam axis, is required. Further space may be required for the construction of biological shielding needed in specific circumstances. Any pretreatment of the neutron beam (polarization filtering or focussing) should be housed in a separate blockhouse with a floor space of approximately $2 \mathrm{~m}$ by $2 \mathrm{~m}$ ( $6.6 \mathrm{ft}$ by $6.6 \mathrm{ft}$ ).

\subsection{Nuclear spectroscopy (WBS 1.4.3.3.2)}

An experimental area of approximately $4 \mathrm{~m}$ by $4 \mathrm{~m}(13.1 \mathrm{ft}$ by $13.1 \mathrm{ft})$, symmetric to the beam axis, is required. Further space may be required for the construction of biological shielding needed in specific circumstances. Any pretreatment of the neutron beam (polarization, filtering or focussing) should be housed in a separate blockhouse with a floor space of approximately $2 \mathrm{~m}$ by $2 \mathrm{~m}(6.6 \mathrm{ft}$ by $6.6 \mathrm{ft}$ ).

\subsection{Isotope separation on-line (WBS 1.4.3.3.3)}

Floor loading capabilities should be compatible with the installation of a large separating magnet, electrostatic detectors, and associated biological shielding. 


\subsubsection{Neutron Interferometer (WBS 1.4.2.8.1)}

The interferometer position must provide the best possible thermal, vibrational, acoustic and seismic isolation. To this end the instrument will be enclosed in its own, environmentally controlled, blockhouse, the floor of which will be decoupled, as far as possible, from the rest of the building. An enclosed area of $8 \mathrm{~m}(26.2 \mathrm{ft})$ long by $6 \mathrm{~m}(19.7 \mathrm{ft})$ wide is required.

\subsubsection{System Configuration and Essential Features}

General layouts for the set of instruments have been developed that meet the requirements of beam spectral range and instrument geometry. Appendix $\mathrm{C}$ of this document contains figures that show these general layouts. Figure 1.1 is the plan view for the ground floor beam room, which contains all instruments on horizontal thermal neutron beam tubes. Figure 1.2 is the plan view for the second floor beam room. Figure 1.3 is the plan view for the guide hall with the instruments on cold guides. Particular features for each instrument are given in the following paragraphs.

\subsubsection{Thermal Nuclear Physics (WBS 1.4.3.1)}

\subsection{Through tube loading station (WBS 1.4.3.1.1)}

The through tube loading station will provide a means of introducing several independent samples into the beam tube. Suitable collimation will prevent some of these sample positions from being directly viewed by the crystal $\gamma$-ray spectrometer. One of the main sample positions should have a mechanism such that the orientation of the sample can be varied over a small range to optimize the resolution when the crystal $\gamma$-ray spectrometer is used under certain geometries.

\subsection{Ultrahigh resolution $\gamma$-spectroscopy (WBS 1.4.3.1.2)}

The crystal $\gamma$-ray spectrometer will consist of three sections:

a fully adjustable collimation system for the $\gamma$ beam emerging from the through tube, a vibration insulated table on which will be mounted the diffracting crystals and associated interferometer optics, and

a translatable table on which a $\gamma$ detector will be mounted.

\subsubsection{Polarized Nuclear Physics (WBS 1.4.3.2)}

\subsection{Precision $\gamma$-ray bolometer (WBS 1.4.3.2.1)}

The neutron beam must be focussed or collimated so that samples of only a few millimeters dimension can be irradiated without activation of surrounding mounting materials, etc.

The location should be compatible with the requirements for operation of a helium dilution refrigerator. (See Sect. 1.2.2.2.1 for structural requirements.) To allow neutrons to impinge on the sample, the refrigerator must be directly coupled to the neutron guide. Provision must be made for periodic interruptions of the neutron beam. Neutrons that do not interact with the sample must be 


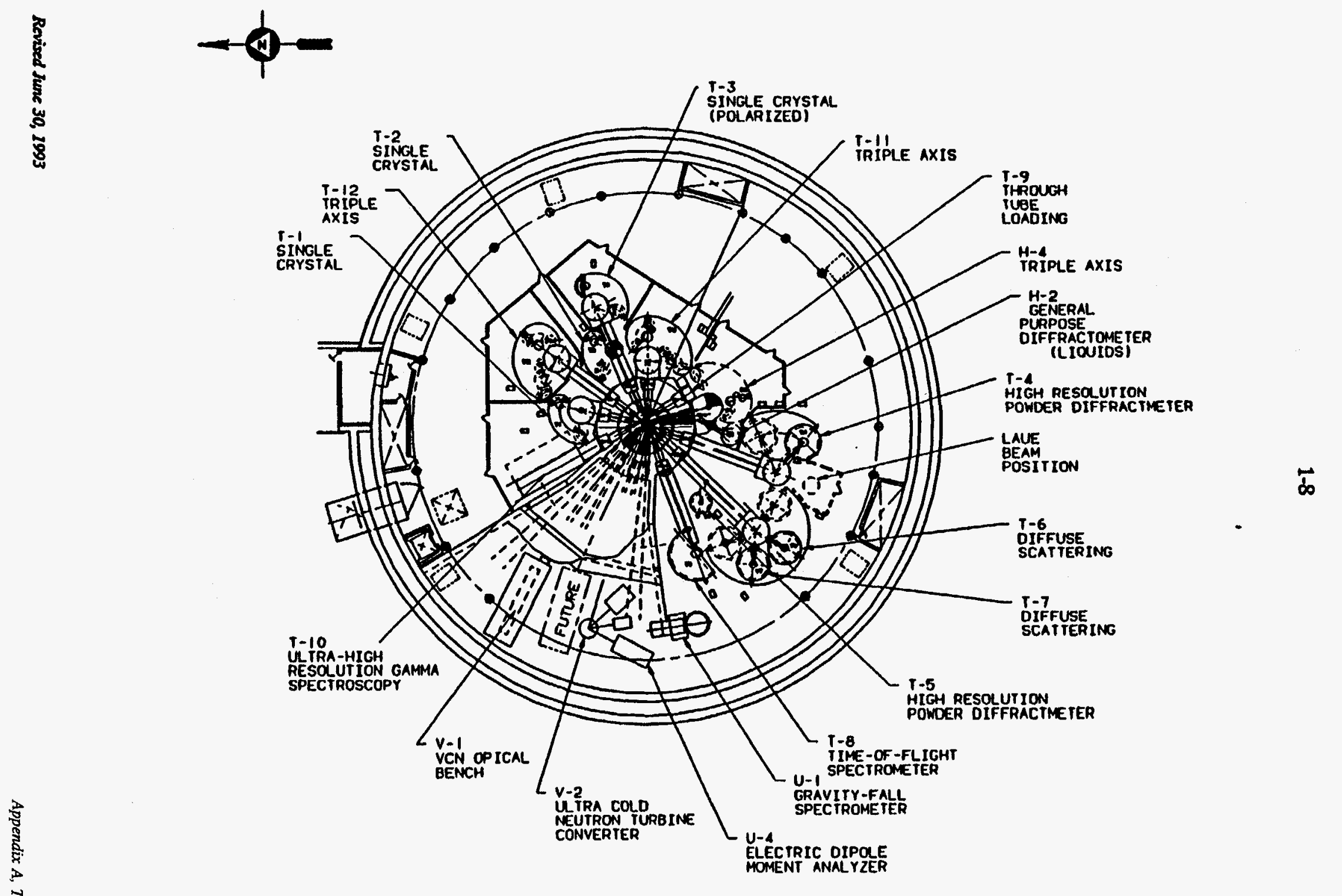

Fig. 1.1. Plan vicw for the ground floor beam room 


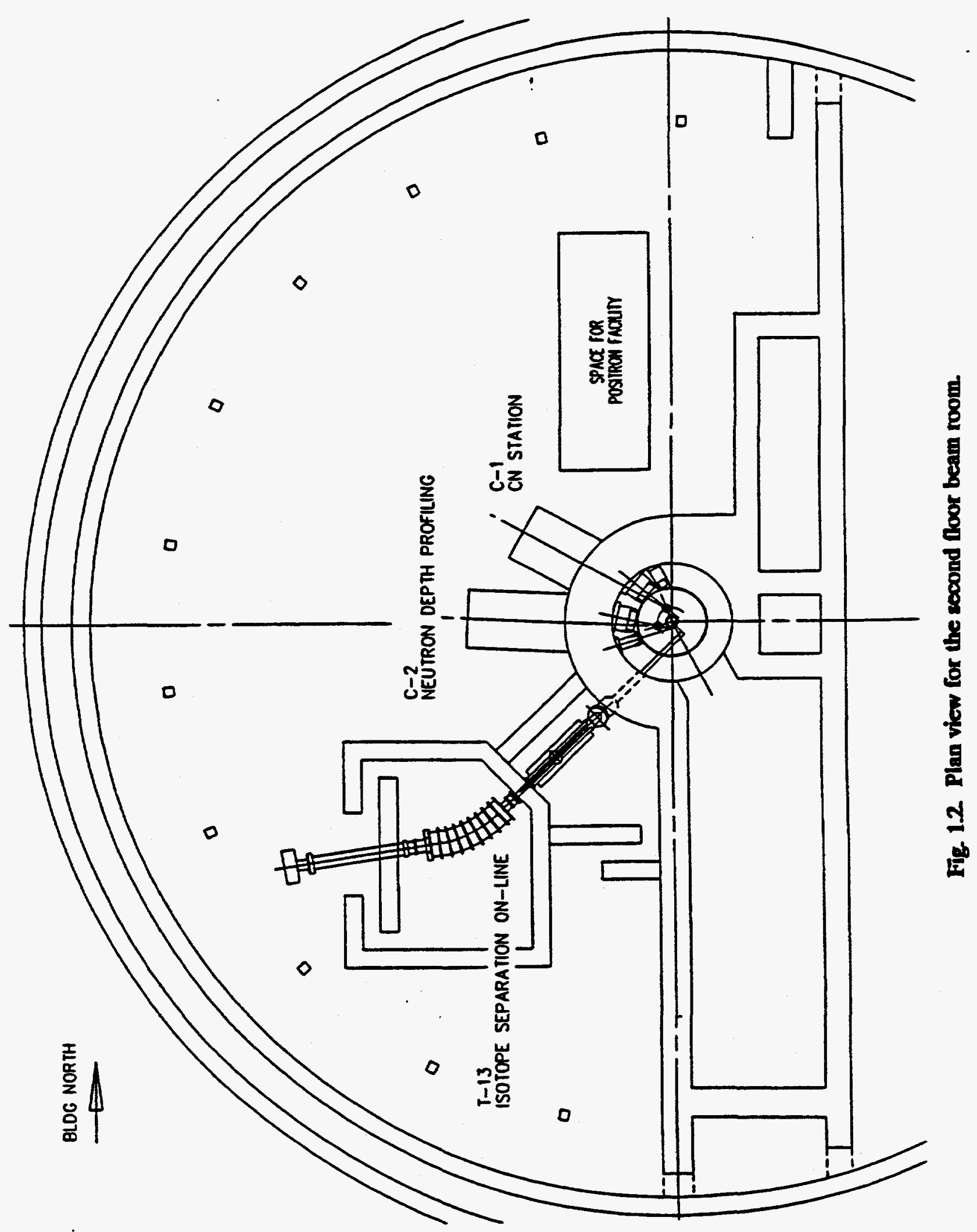

Appendix A, TM-13089 


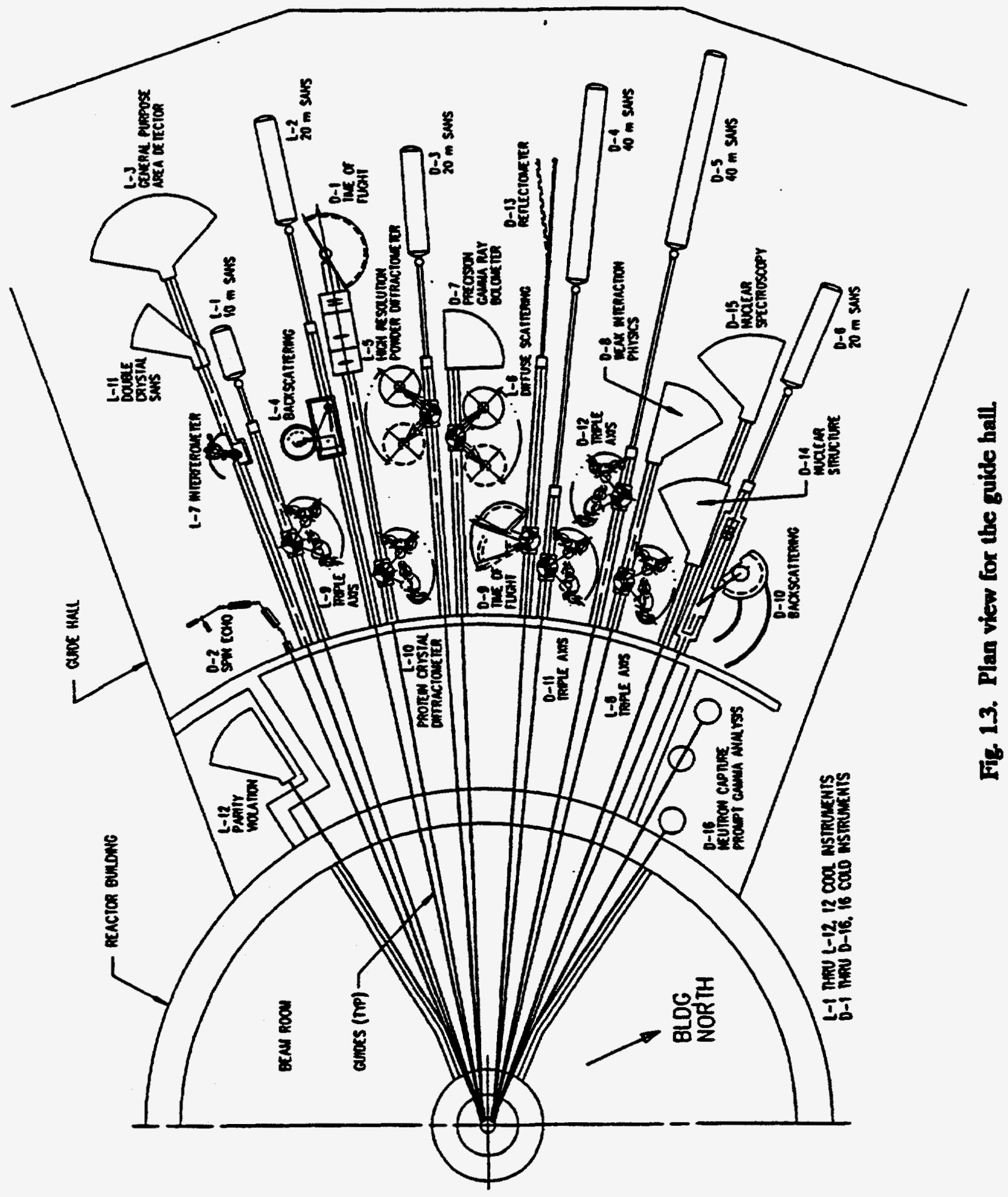


allowed to leave the immediate environment before being absorbed by other materials (i.e., a remote beam dump must be provided).

\subsection{Weak interaction physics (WBS 1.4.3.2.2)}

After passage through a user-supplied setup, any remaining neutrons should be directed to a remote beam dump. Because of the varying geometries of experiments performed at this station, neutron guides of various lengths should be available.

\subsection{Parity violation (WBS 1.4.3.2.3)}

After passage through a user-supplied setup, any remaining neutrons should be directed to a remote beam dump. Because of the varying geometries of experiments performed at this station, neutron guides of various lengths should be available.

\subsubsection{General Nuclear Physics (WBS 1.4.3.3)}

\subsection{Nuclear structure (WBS 1.4.3.3.1)}

Adjustable collimation of the neutron beam should be provided. Because the sample will be placed directly in this collimated beam, an externally adjustable sample mounting should also be provided. An adaptable skeletal framework for the mounting of varying detector types, numbers, and geometries must be available.

\subsection{Nuclear spectroscopy (WBS 1.4.3.3.2)}

Adjustable collimation of the neutron beam should be provided. Because the sample will be placed directly in this collimated beam, an externally adjustable sample mounting should also be provided. An adaptable skeletal framework for the mounting of varying detector types, numbers, and geometries must be available.

After passage through the target area, the emerging neutron beam should be directed to a remote beam dump.

\subsection{Isotope separation on-line (WBS 1.4.3.3.3)}

The ISOL facility will consist of three identifiable sections:

- an ion source located in the neutron beam, consisting of milligram quantities of fissile material and a means to ionize and extract the fission products;

- a stage that will separate the ions according to their mass-to-ionic-charge ratio; and

- an external station to which the mass-separated beam will be delivered. At this point an option should be provided either to conduct experiments directly at this point or to transport the collected ions to an "off-line" experimental station.

The whole facility must be located such that it is possible to extract the beam from the building for use as a neutron rich radioactive ion beam (RIB) for injection into a heavy ion accelerator. 


\subsubsection{Neutron Interferometer (WBS 1.4.2.8.1)}

\subsection{Neutron monochromator}

Neutrons of the desired energy/wavelength will be selected from the spectrum of neutrons in the guide by reflection from an oriented crystal. This monochromatic beam will then be directed to the interferometer position by reflection from a crystal oriented further along the guide, thus producing a monochromatic beam, parallel to the original white beam, which will be delivered to the neutron interferometer position.

\subsection{Interferometer enclosure}

The monochromatic neutron beam will enter the controlled environment of the interferometer enclosure. Collimation of the beam may be necessary before it reaches the interferometer proper. To ensure minimum environmental disruption of the interferometer, several nested stages of isolation (passive and active) will be employed to eliminate thermal gradients and vibrational/seismic disturbance.

\subsubsection{Maintenance}

The nuclear and fundamental physics instruments will be designed to minimize maintenance and spare parts inventory.

\subsubsection{Scheduled Maintenance}

Equipment that operates for a fixed number of hours or a fixed number of operations between scheduled maintenance periods will be configured to minimize redundant operations whenever the increased capital cost of so doing is offset by the reduced maintenance cost. All maintenance will be scheduled to take place during the normal reactor shutdowns unless it specifically requires a neutron beam for testing.

\subsubsection{Unscheduled Maintenance}

Unscheduled maintenance and repair will be minimized by undertaking regular scheduled maintenance and by using well-tested standard designs wherever possible. Downtime for unscheduled maintenance will be minimized by maximizing the use of interchangeable standard components.

\subsubsection{Sample Preparation Area}

An area of $10 \mathrm{~m}^{2}\left(107.6 \mathrm{ft}^{2}\right)$ will be provided near each instrument for final sample preparation and small maintenance operations. A standard workbench and service package will be provided in this area.

In addition, the nuclear physics personnel will require access to a laboratory equipped to handle low-activity radioisotopes and to perform simple chemical and mechanical operations on all samples. 


\subsubsection{Surveillance and In-Service Inspection}

All instrumental areas should be equipped with standard $\gamma$-ray and neutron monitors for background or sample-connected activity. In addition, all exhaust gases coming from each area should be monitored for increased activity resulting from possible loss of sample integrity.

\subsubsection{Thermal Nuclear Physics (WBS 1.4.3.1)}

\subsection{Through tube loading station (WBS 1.4.3.1.1)}

Surveillance of sample loading and unloading should be provided by a comprehensive system of TV cameras connected to a central monitoring location. The helium gas egressing from the through tube should be monitored at all times for radioactive contamination, which could indicate disintegration of the sample or loss of integrity of the internal atmosphere.

\subsection{Ultrahigh resolution $\gamma$-spectroscopy (WBS 1.4.3.1.2)}

The experimental equipment will also provide a means of monitoring sample integrity, etc., which would be indicated by sudden changes in counting rates or in resolution.

To prevent excessive disturbance of the controlled environment of the crystal $\boldsymbol{\gamma}$-ray spectrometer, TV monitoring equipment should be employed.

\subsubsection{Polarized Nuclear Physics (WBS 1.4.3.2)}

\subsection{Precision $\gamma$-ray bolometer (WBS 1.4.3.2.1)}

Regular logging of conditions at various locations within the helium dilution refrigerator will serve to identify any possible loss of liquid refrigerant, vacuum leaks, or blockages in circulatory systems.

\subsection{Weak interaction physics (WBS 1.4.3.2.2)}

No special requirements are needed other than standard monitoring of $\gamma$-ray and neutron background.

\subsection{Parity violation (WBS 1.4.3.2.3)}

The earliest possible detection of liquid hydrogen leaks in this area is essential.

\subsubsection{General Nuclear Physics (WBS 1.4.3.3)}

\subsection{Nuclear spectroscopy (WBS 1.4.3.3.1)}

Experimental count rates will serve as additional monitors of sample integrity. 


\subsection{Nuclear structure (WBS 1.4.3.3.2)}

Experimental count rates will serve as additional monitors of sample integrity.

\subsection{Isotope separation on-line (WBS 1.4.3.3.3)}

Experimental count rates will serve to monitor the continuing optimization of the mass separator stage performance.

\subsubsection{Neutron Interferometer (WBS 1.4.2.8.1)}

Experimental count rates will serve to monitor the correct functioning of the instrument.

\subsubsection{Instrumentation and Control}

Overall control of each instrument will be provided by a dedicated autonomous computer that is interfaced to the electromechanical components of the instrument to provide remote setting and readout of motors, magnets, and sample environment parameters. Software will be capable of computing or storing scan settings, performing scans, data-logging, data analysis, and monitoring safe operations. The hardware interfaces will include limit sensing and safety interlocks to the beam control system.

\subsubsection{Interfacing Systems}

A standard set of services is required at each nuclear and fundamental physics station.

\subsubsection{Electrical}

All power sources required for instrument operation will be stoppable from a single pushbutton, which will be clearly labelled as a safety cutout, easily visible, and quickly accessible.

\subsection{Industrial power}

Industrial power will be provided as needed to run motorized equipment and for general purpose use.

\subsection{Clean power}

Clean power will be provided to run non-safety-related electronic interfaces in which electrical noise would compromise data or reliability. To preserve the integrity of the supply, clean power will be hard-wired or available through special connectors only.

\subsection{Uninterruptible power}

Uninterruptible power will be provided to the instrument control computer and interfaces whose failure could result in an unsafe local condition, such as safety interlocks or temperature control 
devices. To preserve the integrity of the supply, uninterruptible power will be hard-wired or available through special connectors only.

\subsubsection{Compressed Gas}

\subsection{Air}

Compressed air access is required for all instruments for the operation of beam shutters and safety interlocks. In addition, compressed air may be used to position individual instrument components on "air pads."

\subsection{Helium}

The through tube and loading station (WBS 1.4.3.1.1) will require a constant supply of helium gas in a circulatory system. If the purity of the "in-house" supply is not sufficient, provision should be made for decoupling this system and substituting an independent, "clean" supply.

Depending on user demand and configuration, the weak interaction physics (WBS 1.4.3.2.2), parity violation physics (WBS 1.4.3.2.3) and neutron interferometer (WBS 1.4.2.8.1) stations may also require access to gaseous helium.

Other nuclear and fundamental physics stations will require gaseous helium only on a very occasional basis.

\subsection{Nitrogen}

All nuclear and fundamental physics stations will require gaseous nitrogen only on very rare occasions.

\subsubsection{Liquid Gases}

\subsection{Helium}

When in operation, the helium dilution refrigerator at the precision $\gamma$-ray bolometer station (WBS 1.4.3.2.1) will require multiliter quantities of liquid helium daily. Depending on user demand and configuration this may also apply to the weak interaction physics (WBS 1.4.3.2.2) and parity violation physics (WBS 1.4.3.2.3) stations.

\subsection{Nitrogen}

All nuclear and fundamental physics stations will require multiliter quantities of liquid nitrogen daily. 


\subsubsection{Vacuum}

All nuclear and fundamental physics stations will require access to rough vacuum 13.3 to $1.3 \mathrm{~Pa}\left(-10^{-1}\right.$ to $10^{-2}$ Torr) facilities. In addition, much harder vacuums $0.13 \mathrm{mPa}\left(-10^{-6} \mathrm{Torr}\right)$ will be needed regularly by the polarized nuclear physics (WBS 1.4.3.2) stations and occasionally also by all other stations.

\subsubsection{Gas Recovery}

\subsection{Vacuum pump exhaust}

This will be required by all nuclear and fundamental physics stations.

\subsection{Helium}

Helium recovery ports must be provided at all polarized nuclear physics (WBS 1.4.3.2) stations.

\subsubsection{Environmental}

The environment provided at each instrument must be compatible with standard research laboratory requirements.

Gamma-ray and neutron backgrounds must be kept ALARA. For the Precision $\gamma$-ray Spectroscopy Station, specific efforts should be made to reduce such background to as low levels as possible.

\subsubsection{Quality Assurance}

Quality assurance requirements for design, procurement, manufacture, installation, inspection, testing, and operation activities shall be in accordance with the requirements for the safety classification specified in ORNL/TM-11446/R1. The quality assurance requirements for each safety class are given in Sect: 4.5 of the Plant Design Requirements (PDR).

\subsubsection{Codes and Standards}

A list of standards, codes, and guides that are considered to be applicable to the design of the experiment systems is given in Appendix $\int$.

\subsubsection{Reliability Assurance}

Reliability will be ensured through training users in correct operational procedures, regular scheduled maintenance, a design that minimizes the number of different components, and using clean power to run critical electronic components. 


\section{DESIGN DESCRIPTION}

This section contains detailed descriptions of the nuclear and fundamental physics instruments proposed for the ANS.

\subsection{SUMMARY}

\subsubsection{Thermal Nuclear Physics (WBS 1.4.3.1)}

\subsubsection{Through Tube Loading Station (WBS 1.4.3.1.1)}

The sample "irradiation assembly" will be capable of holding several independent samples simultaneously. These will be accessed from the loading station. The holder will be inserted into the tangential through tube such that the samples can be placed in various neutron fluxes. On removal, active samples will be stored or disposed of appropriately.

\subsubsection{Ultrahigh Resolution $\gamma$-Spectroscopy (WBS 1.4.3.1.2)}

This facility will be located at the opposite end of the tangential through tube from the loading station and will consist of a crystal $\gamma$-ray spectrometer and a pair formation $\gamma$-ray spectrometer.

The crystal $\gamma$-ray spectrometer will be based on a new design currently under development at Institut Laue-Langevin (ILL). This arrangement will allow for the device to be configured in three geometries:

a single bent-crystal geometry

a double bent-crystal geometry

a double flat-crystal geometry

In all cases wavelength selection from a collimated $\gamma$-ray beam is performed by Bragg diffraction (in transmission) by an appropriate arrangement of crystals. The diffracted $\gamma$ rays are detected by a separate $\gamma$ detector.

\subsubsection{Polarized Nuclear Physics (WBS 1.4.3.2)}

\subsubsection{Precision $\gamma$-Ray Bolometer (WBS 1.4.3.2.1)}

The bolometer system will consist of a helium dilution refrigerator within which samples can be cooled to milliKelvin temperatures. It will have a side access port coupled directly to a neutron guide to allow neutrons to reach the sample. To allow for rapid sample exchange, a top-loading facility will also be included.

\subsubsection{Weak Interaction Physics (WBS 1.4.3.2.2)}

This station will be left open for user-supplied equipment. 


\subsubsection{Parity Violation (WBS 1.4.3.2.3)}

This station will be left open for user-supplied equipment.

\subsubsection{General Nuclear Physics (WBS 1.4.3.3)}

Although the three general nuclear physics installations will be used for very different applications, it is likely that all will make use of the same techniques to determine nuclear radiation energies, intensities, and coincidence relationships. Therefore, a general pool of interchangeable detectors for these functions is foreseen.

\subsubsection{Nuclear Structure (WBS 1.4.3.3.1)}

The basic installation will be a skeletal framework for the mounting of $\gamma$-ray and particle detectors from the general pool (see WBS 1.4.3.3). These detectors will view a central target placed in the neutron beam.

\subsubsection{Nuclear Spectroscopy (WBS 1.4.3.3.2)}

The basic installation will be a skeletal framework for the mounting of $\gamma$-ray and particle detectors from the general pool (see WBS 1.4.3.3). These detectors will view a central target placed in the neutron beam.

\subsubsection{Isotope Separation On-Line (WBS 1.4.3.2.3)}

Ionically charged fission products recoiling from fission events will enter an electromagnetic device that will separate them according to mass. The separated beam will be brought to a station where the ions can be accumulated for nuclear structure studies or implanted into hosts for solid state experiments. Further developments may see this beam itself being injected into a remote heavy ion accelerator.

For nuclear structure studies the basic installation will be a skeletal framework for the mounting of $\gamma$-ray and particle detectors from the general pool (see WBS 1.4.3.3).

\subsubsection{Neutron Interferometer (WBS 1.4.2.8.1)}

The neutron interferometer station will consist of a double-crystal monochromator inserted into the white neutron beam to provide a parallel beam of monoenergetic neutrons. This beam will be delivered to an environmentally isolated enclosure for use in the neutron interferometer.

The interferometer itself will be environmentally and vibrationally isolated from changes and/or activity in the immediate vicinity of the enclosure. 


\subsection{DETAILED SYSTEM DESCRIPTION}

\subsubsection{Thermal Nuclear Physics (WBS 1.4.3.1)}

\subsubsection{Through Tube Loading Station (WBS 1.4.3.1.1)}

\subsection{Loading station}

Samples will be introduced into the through tube loading station via an interlock to maintain the integrity of the internal inert atmosphere. Operations on samples will then be carried out via remote handling devices. Different sample holder designs will all interface in the same way with the remote handling devices. Within the through tube loading station will be a work area, designated for sample manipulation into which various components can be maneuvered as desired.

For the loading of new samples and the extraction of active old samples, the complete sample irradiation assembly will be brought into the work area. Individual samples may then be manipulated as desired. With the sample irradiation assembly withdrawn, there will be access to a chute for the disposal of active samples.

In the event that retention of an active sample is required, a storage assembly will be brought into the work area to receive the sample. This assembly will then be withdrawn to a shielded area near the work area. The storage assembly will be capable of retaining up to six individual samples of varying design (TBD) [HOLD].

\subsection{Sample irradiation assembly}

The through tube loading station will interface with the tangential through tube so that the sample irradiation assembly can be introduced to, and withdrawn from, the through tube. Following the insertion of the irradiation assembly into the through tube, this interface will be sealed and shielded. The irradiation assembly will be stopped at a position in the through tube corresponding to the neutron flux desired for sample irradiation.

The sample irradiation assembly will be constructed from graphite to minimize background radiation. It will be configured such that several samples may be irradiated simultaneously. Two of these sample positions are intended only for the "breeding" of neutron-rich isotopes in situ to be used for subsequent study with ultrahigh resolution $\gamma$-spectroscopy. These positions will be shielded, by suitable collimation, from direct view by the $\gamma$-spectrometers. The other sample positions will be collinear with the axis of the through tube to be viewed directly by the $\gamma$-ray spectrometers. A minimum of five collinear sample positions is required so that multigram quantities of sample material may be irradiated.

One of the main sample positions will be controlled remotely so that the orientation of the sample can be adjusted around two perpendicular axes. This adjustment is necessary only when the crystal $\gamma-$ ray spectrometer is configured in a particular geometry.

\subsection{Through Tube}

During normal operation the through tube will contain an atmosphere of gaseous helium at a pressure of $100 \mathrm{kPa}(1 \mathrm{~atm})$. Particular experiments will require that this pressure be variable from $500 \mathrm{~Pa}$ ( $5 \mathrm{mbar}$ ) to $200 \mathrm{kPa}(2 \mathrm{~atm})$. The helium will be continually cycled through the tube, and the 
egressing gas will be monitored for signs of increased activity, which may indicate loss of integrity of a sample or other contamination. This circulation system will also interface with the through tube loading station to ensure integrity of the internal atmosphere during sample manipulation.

Lead $(\mathrm{Pb})$ collimators will be located within the through tube to ensure that only a narrow beam of intense $\gamma$ radiation is delivered to the ultrahigh resolution $\gamma$-ray spectrometers. At this end the through tube will be sealed with only a thin window to maintain the integrity of the internal atmosphere while allowing the passage of low-energy $(-30 \mathrm{keV}) \gamma$ radiation.

\subsubsection{Ultrahigh Resolution $\gamma$-Ray Spectroscopy (WBS 1.4.3.1.2)}

The ultrahigh resolution $\gamma$-ray spectroscopy station will house two distinct instruments. These will view the same samples, which will be located in the tangential through tube. Possible experiments will utilize either one or both of these instruments.

For maximum flexibility, a fully adjustable collimator for the $\gamma$-ray beam will be located at the exit of the through tube. This will be adjustable in both absolute horizontal and vertical placement and also in the width and height of the opening.

\subsection{Crystal $\gamma$-ray spectrometer}

The crystal $\gamma$-ray spectrometer will consist of three distinct parts. The first will be a vibrationinsulated base platform on which will be located a separate, moveable table that will accommodate the diffracting crystals, their rotation mechanisms, and the laser-based optical interferometry systems needed for controlling the crystal orientations. The elevation and inclination of the base platform will be continuously monitored to ensure that the diffracting planes of the crystals are kept vertical.

The crystal table will be located on air pads so that its position and rotation, relative to the $\gamma$-ray beam axis, can be easily varied. On this table, approximately $500 \mathrm{~mm}$ (19.7 in.) apart, will be located two mounts for diffracting crystals. These mounts will be connected to mechanical systems that allow precise rotation about a vertical axis. These rotations will be monitored and set using polarization-sensitive Michelson interferometer systems interacting with optical elements at the ends of rigid arms fixed to the rotational axes. The optical elements of the interferometers (including the laser) will also be mounted on the crystal table.

The crystal mounts themselves will accommodate either flat or bent crystals, depending on the desired instrument geometry. Fine adjustment of the vertical alignment of the crystal planes will be made mechanically on the crystal mounts.

A $\gamma$-ray detector will be mounted behind the main platform. The mounting for this detector will allow both translation and rotation so that the detector itself may intercept the diffracted $\gamma$ beam. Because many experiments will involve very low counting rates, the $\gamma$ detector will be heavily shielded from both $\gamma$ and neutron backgrounds. In addition, the small diffraction angles involved at high $\gamma$ energies will require additional collimation of the diffracted $\gamma$ beam between the crystal table and the detector. This collimation will also be maneuverable to allow the $\gamma$ detector a clear view of the first or second diffraction crystal, depending on the particular instrument geometry in use.

Because of its sensitivity to changes in climate and environment, the whole spectrometer will be housed in a sealed and acoustically insulated blockhouse. The internal environment will be closely controlled and monitored. 


\subsection{Pair formation $\gamma$-ray spectrometer}

The principle of operation of this spectrometer is based on the $\mathrm{e}^{+} \mathrm{e}^{-}$pair formation mechanism for the interaction of $\gamma$ rays of energy higher than $1.02 \mathrm{MeV}$ with matter. The $\mathrm{e}^{+}$, after thermalization, will annihilate to give two antiparallel $\gamma$ rays of $511 \mathrm{keV}$ energy each. If the initial interaction takes place in one detector while the two $511-\mathrm{keV} \gamma$ rays are detected in other detectors that surround the first, a unique triple-coincidence signature results.

The central detector of the pair formation $\gamma$-ray spectrometer will be mounted co-axially with the collimated $\gamma$ beam. It will be immediately preceded by adjustable collimation so that the number of $\gamma$ rays reaching the detector per second can be adjusted to optimize performance. The central detector will be surrounded by further detectors, with no direct view of the $\gamma$-beam. The purpose of these is to detect the $511 \mathrm{keV} \gamma$ rays coming from the $\mathrm{e}^{+}$annihilation in the central detector.

The whole assembly will be shielded from both $\gamma$-ray and neutron background and will be housed in its own separate blockhouse.

\subsubsection{Polarized Nuclear Physics (WBS 1.4.3.2)}

\subsubsection{Precision $\gamma$-Ray Bolometer (WBS 1.4.3.2.1)}

Samples to be cooled to $5 \mathrm{mK}$ will be attached to the cold finger of a helium dilution refrigerator. Such devices are available from a number of commercial sources.

To allow neutrons to reach the sample, the cryostat of the refrigerator will have a side access port coupled directly to a neutron beam line. Internal, adjustable, collimation will ensure that only a narrow neutron beam is allowed to enter the cryostat itself. A small station in the neutron beam line, ahead of the cryostat access port, will allow the insertion of equipment for optional polarization, filtering, or chopping of the neutron beam.

To make possible the rapid changing of samples, the refrigerator will also be provided with a toploading facility. By using this mechanism, new samples are rapidly precooled with liquid refrigerants and are then inserted directly into the low-temperature region.

Neutrons that pass into the cryostat but do not interact with the sample will be allowed to exit through a similar exit port and led to a remote beam dump.

\subsubsection{Weak Interaction Physics (WBS 1.4.3.2.2)}

This station will be left open for user-supplied equipment. A small blockhouse preceding this station will house equipment for optional polarization, filtering, or chopping of the neutron beam.

\subsubsection{Parity Violation (WBS 1.4.3.2.3)}

This station will be left open for user-supplied equipment. A small blockhouse preceding this station will house equipment for optional polarization, filtering or chopping of the neutron beam. This experimental area will be isolated from the rest of the neutron guide hall since certain experiments may require the use of multiliter quantities of liquid hydrogen. 


\subsubsection{General Nuclear Physics (WBS 1.4.3.3)}

All general nuclear physics installations will make use of $\gamma$-ray and particle detectors. Such detectors are commercially available and a general pool of these will be established. Details will be given in Sect. 2.5.3.

\subsubsection{Nuclear Structure (WBS 1.4.3.3.1)}

Adjustable collimation will define a neutron beam to be delivered to an evacuated sample chamber. Optional polarization, filtering, or chopping of the beam will be provided. The sample chamber will be at the center of a flexible skeletal framework into which will be mounted detectors from the general pool.

Particle detectors that require direct access to the evacuated sample chamber will be accommodated by having a number of chambers with custom access ports. These chambers will be lined to minimize the escape of sample-scattered neutrons, which may damage the surrounding detectors.

\subsubsection{Nuclear Spectroscopy (WBS 1.4.3.3.2)}

Adjustable collimation will define a neutron beam to be delivered to an evacuated sample chamber. Optional polarization or chopping of the beam will be provided. The sample chamber will be at the center of a flexible skeletal framework into which detectors from the general pool will be mounted.

Particle detectors that require direct access to the evacuated sample chamber will be accommodated by a number of different chambers with custom access ports. These chambers will be lined to minimize the escape of sample-scattered neutrons, which may damage the surrounding detectors.

\subsubsection{Isotope Separation On-Line (WBS 1.4.3.3.3)}

\subsection{Production of fission products}

Milligram quantities of fissile material will be introduced into a neutron beam via a shielded cask. The ionized fission fragments will be guided to the mass separator using a system of magnetic lenses.

\subsection{Separation of fission products}

On arrival at the mass separator stage, the charged fission products will enter a planar region between the poles of a large electromagnet and then between the plates of a large condensor. By suitably adjusting the magnetic and electric field strengths, the desired mass can be guided to an exit port. Magnetic lenses will then guide the selected ions to a measurement station.

\subsection{Measurement station}

At the measurement station a number of options will be available. 
The ion beam could be stopped at the center of a flexible skeletal framework into which will be mounted detectors from the general pool. Particle detectors that require direct access to the evacuated sample chamber will be accommodated by a number of different chambers with the custom access ports.

The ion beam could be deposited on a tape transport system to be transported to an off-line study position.

The ion beam could be implanted in a solid sample for later study of solid state effects resulting from implanted radioactive ions.

The ion beam could be directed to a heavy ion accelerator for acceleration to higher energies thus providing a source of neutron-rich RIBs.

\subsubsection{Neutron Interferometer (WBS 1.4.2.8.1)}

\subsubsection{Double-Crystal Monochromator}

An appropriately cut crystal will be inserted in the white neutron beam within a neutron guide. To maximize efficiency this crystal will have dimensions such that it will cover the whole neutron beam area. The orientation of the crystal will be varied to select, by diffraction in reflection, the desired neutron energy/wavelength. This diffraction beam will be intercepted by a second, identical crystal oriented at the same angle, which thus will re-reflect the monochromatic neutron beam such that it is parallel to the initial white beam but laterally displaced.

\subsubsection{Neutron Interferometer}

The laterally displaced monochromatic neutron beam will enter the interferometer enclosure through a small window. The environment in the enclosure will be closely controlled and monitored.

The floor on which the interferometer stands will be decoupled from that of the rest of the guide hall and a separate platform, supported only on pneumatic springs, will be installed. Further vibration isolation will be provided by mounting the interferometer on a second platform, the supports for which will provide a second, active, isolation system via a servofeedback mechanism. This second platform will be enclosed by a moveable noise and thermal shield. Active stabilization will be measured relative to an inertial test mass.

The interferometer itself will consist of a set of crystals used to split, diffract, and recombine the monochromatic neutron beam after passage of one "arm" through a sample. The intensity of the recombined beam will be measured with a standard neutron detector.

\subsection{SYSTEM PERFORMANCE CHARACTERISTICS}

\subsubsection{Thermal Nuclear Physics (WBS 1.4.3.1)}

\subsubsection{Through Tube Loading Station (WBS 1.4.3.1.1)}

Under normal operation samples will be introduced to the inert atmosphere of the loading station via on interlock. For air-sensitive samples it will be necessary to have a separate sealed container interfaced with this interlock. All sample manipulation within the loading station will be carried out 
using remote mechanical arms. However, provisions will be made for manual intervention in the event of system failure or accident.

After the introduction of the sample irradiation assembly into the through tube and the closure of the loading station/through tube interface, the internal atmosphere of helium gas will be circulated via the main helium circuit. Under normal circumstances the internal atmosphere will be maintained at a pressure of just under $100 \mathrm{kPa}(1 \mathrm{~atm})$. Special circumstances may require pressures as low as $500 \mathrm{~Pa}$ ( 5 mbar) or up to $200 \mathrm{kPa}(2 \mathrm{~atm})$. In all cases gas exiting from the through tube will be monitored for increased radioactivity before it is reintroduced to the main helium circuit.

For certain low counting rate experiments, it will be necessary to replace the main helium supply with a source of ultra-pure helium.

The insertion and withdrawal of the sample irradiation assembly will proceed over a time scale of approximately $15 \mathrm{~min}$ to eliminate excessive thermal gradients. Exact times will depend on the desired irradiation position.

Once in position mechanical drives will allow the rotation of one sample position by $\pm 10^{\circ}$ (in steps of $0.1^{\circ}$ ) about the horizontal axis coaxial with the through tube and also about the vertical axis. Such adjustments will only be necessary if the crystal $\gamma$-ray spectrometer is being used in its single bent crystal geometry.

\subsubsection{Ultrahigh Resolution $\gamma$-Spectroscopy (WBS 1.4.3.1.2)}

\subsection{Crystal $\gamma$-ray spectrometer}

The performance characteristics of the crystal $\gamma$-ray spectrometer will depend on the particular operating geometry.

In double flat-crystal mode, energy resolutions of a few parts per million are expected. This is achieved by measuring diffraction angles with a precision of 1 arc second and by using ultra-pure diffraction crystals. The energy range for the spectrometer in this mode will be from $30 \mathrm{keV}$ to several hundred femto Joules (million electron volts). However, the sharp reduction in efficiency that occurs with increasing energy will limit practical measurements to energies less than $-5 \mathrm{MeV}$. This geometry will be used to measure absolute $\gamma$-ray wavelengths and narrow Doppler profiles. The low efficiency of the spectrometer will mean that only approximately one in every $10^{12} \gamma$ rays leaving the sample will be detected.

In double bent-crystal mode, energy resolutions of 10 to $20 \mathrm{ppm}$ are expected with an increase in efficiency of two to three orders of magnitude over the flat-crystal geometry.

Energy range and limitations are the same as for the double flat-crystal geometry above. This will be the normal operating mode for nuclear structure experiments.

In the single bent-crystal geometry, the energy resolution will depend critically on sample preparation. However, typical values will be several hundred parts per million. The absolute efficiency will be increased by a factor of two over that of the double bent crystal. Because of the decreased resolution, the useful energy range for this geometry will be from $50 \mathrm{keV}$ to approximately $1.5 \mathrm{MeV}$. The principal use of this mode will be to scan simple $\gamma$-ray spectra and to identify regions in more complex spectra that will require more detailed study using the highresolution geometries. 


\subsection{Pair formation $\gamma$-spectrometer}

This spectrometer will measure $\gamma$-ray spectra in the energy range from 2 to $10 \mathrm{MeV}$. Using commercially available technology, energy resolutions of $2 \mathrm{keV}$ at $2 \mathrm{MeV}$ and $6 \mathrm{keV}$ at $10 \mathrm{MeV}$ are possible. The resolution of such systems deteriorates with increasing counting rate, and thus adjustable collimation will be used to limit the number of $\gamma$ rays reaching the detector to $10^{4} / \mathrm{s}$. Of these only $-1 \%$ will produce the characteristic triple-coincidence signature, thus making the useful counting rate approximately $100 / \mathrm{s}$.

\subsubsection{Polarized Nuclear Physics (WBS 1.4.3.2)}

\subsubsection{Precision $\gamma$-Ray Bolometer (WBS 1.4.3.2.1)}

Under normal operating conditions, with neutrons impinging on the sample, the helium dilution refrigerator will maintain the sample at a temperature of below $10 \mathrm{mK}$. With the neutron access port closed the system will maintain a temperature of $5 \mathrm{mK}$ (or below) continuously.

The cooling power of the refrigeration stage will be commensurate with technology at the time of construction. (Currently, such technology is advancing rapidly and no definite figures can be given.) Samples loaded into the refrigerator will attain base temperature in a time determined by the cooling power. (Currently, this time is a few hours.)

\subsubsection{Weak Interaction Physics (WBS 1.4.3.2.2)}

System performance characteristics will be determined by user-supplied equipment.

\subsubsection{Parity Violation (WBS 1.4.3.2.3)}

System performance characteristics will be determined by user-supplied equipment.

When liquid hydrogen is in use, all relevant equipment will be monitored for leaks using standard industrial practices for handling this material. Automatic shutdown of all equipment will be initiated, and audible and visual alarms will be activated if such leaks are detected.

\subsubsection{General Nuclear Physics (WBS 1.4.3.3)}

\subsubsection{Nuclear Structure (WBS 1.4.3.3.1)}

In the most general case the sample chamber, directly attached to the neutron guide, will operate under a rough vacuum of $1.3 \mathrm{~Pa}\left(10^{-2}\right.$ Torr). In cases in which particle detectors must be mounted inside the sample chamber, vacuums of as low as $0.13 \mathrm{mPa}\left(10^{-6}\right.$ Torr $)$ may be needed.

The usual limitation in such experiments is imposed by the degradation of the energy resolution of $\gamma$-ray detectors with high counting rates $(>10,000$ counts per second). Variable collimation of the incoming neutron beam will allow for the optimum adjustment of these counting rates.

Another important factor is the damage that can be caused to certain types of detector if neutrons are allowed to enter them. To minimize this effect all neutrons scattered from the sample and its mounting will be absorbed within the sample chamber itself by an inner coating of ${ }^{6} \mathrm{Li}$. 


\subsubsection{Nuclear Spectroscopy (WBS 1.4.3.3.2)}

In the most general case the sample chamber, directly attached to the neutron guide, will operate under a rough vacuum of $1.3 \mathrm{~Pa}\left(10^{-2} \mathrm{Torr}\right)$. In cases in which particle detectors must be mounted inside the sample chamber, vacuums of as low as $0.13 \mathrm{mPa}\left(10^{-6}\right.$ Torr) may be needed.

The usual limitation in such experiments is imposed by the degradation of the energy resolution of $\gamma$-ray detectors with high counting rates $(>10,000$ counts per second). Variable collimation of the incoming neutron beam will allow for the optimum adjustment of these counting rates.

Another important factor is the damage that can be caused to certain types of detectors if neutrons are allowed to enter them. To minimize this effect all neutrons scattered from the sample and its mounting will be absorbed within the sample chamber itself using an inner coating of ${ }^{6} \mathrm{Li}$.

\subsubsection{Isotope Separation On-Line (WBS 1.4.3.3.3)}

The fissile material within the ion source assembly will be configured such that the escape of the ionized fission products is allowed so that they may be transported to the mass-separation stage. The transit time from formation to separation will be minimized by using a system of electromagnetic accelerators and lenses to guide the products.

The separation stage will be capable of producing individual mass chains that have high resolving power. The stabilization of the separator magnetic field will be commensurate with maintaining this resolution. To ensure proper operation the whole system must be run with a vacuum of at most $0.13 \mathrm{mPa}\left(10^{-6}\right.$ Torr $)$.

Under normal operation a tape transport system will be installed at the post separation experimental station. This system will also be coupled to the main vacuum of the separator.

\subsubsection{Neutron Interferometer (WBS 1.4.2.8.1)}

\subsubsection{Double-Crystal Monochromator}

The monochromator must be capable of selecting neutrons with wavelengths in the range from 0.13 to $0.55 \mathrm{~nm}\left(1.3\right.$ to $5.5 \AA$ ), corresponding to minimum and maximum reflection angles of $40^{\circ}$ and $110^{\circ}$ respectively. (It will be necessary to insert different crystals to achieve these extrema.)

The final diffracted beam must be delivered to the neutron monochromator at the same spot, independent of wavelength, within a tolerance of (TBD).

\subsubsection{Neutron Interferometer}

The temperature within the interferometer enclosure must be stable to within a few millidegrees. Also, the nested vibration isolation system must reduce acoustic and seismic disturbances to the minimum practical level. 


\subsection{SYSTEM ARRANGEMENT}

Schematic diagrams for all relevant instruments and configurations are contained in Appendix C.

\subsubsection{Thermal Nuclear Physics (WBS 1.4.3.1)}

\subsubsection{Through Tube Loading Station (WBS 1.4.3.1.1)}

The through tube loading station will be located at one end of the tangential through tube. Within the station there will be a shielded area into which the sample irradiation assembly can be withdrawn when sample manipulations are to be carried out.

Interfaced with the sample manipulation area will be

an interlock for the introduction of samples from the outside, an area in which irradiated samples can be stored for future use, and a facility for the disposal of active samples.

Whenever the sample irradiation assembly is either fully extracted from or fully inserted into the through tube, the interface between the tube and the working area will be sealed and shielded.

A schematic diagram of all these configurations is given in Fig. 2.1.

Sample manipulations will be carried out using closed-circuit TV and remote mechanical arms controlled from an adjacent control panel.

Other functions to be controlled from this panel are

the circulation of gaseous helium through the loading station and through tube, the insertion and extraction of the sample irradiation assembly into the through tube, rotation of sample position (if desired), and monitoring of exit gases for increased radioactivity.

\subsubsection{Ultrahigh Resolution $\gamma$-Spectroscopy (WBS 1.4.3.1.2)}

Gamma rays from samples within the reactor will pass through a variable collimator and enter the spectroscopy station.

\subsection{Crystal spectrometer}

The $\gamma$ beam will impinge on the front crystal of the spectrometer, which will select a narrow band of energies. The rest of the intense $\gamma$ beam will proceed and will be intercepted by heavy lead shielding at a convenient point.

The two crystals of the spectrometer will be mounted on an isolated table within an environmentally stable enclosure. After diffraction, the selected $\gamma$-rays will be detected by using a standard $\gamma$ detector, which will be heavily shielded against scattered neutron and $\gamma$ background.

The possible geometries for the crystal spectrometer are shown in Fig. 2.2. 


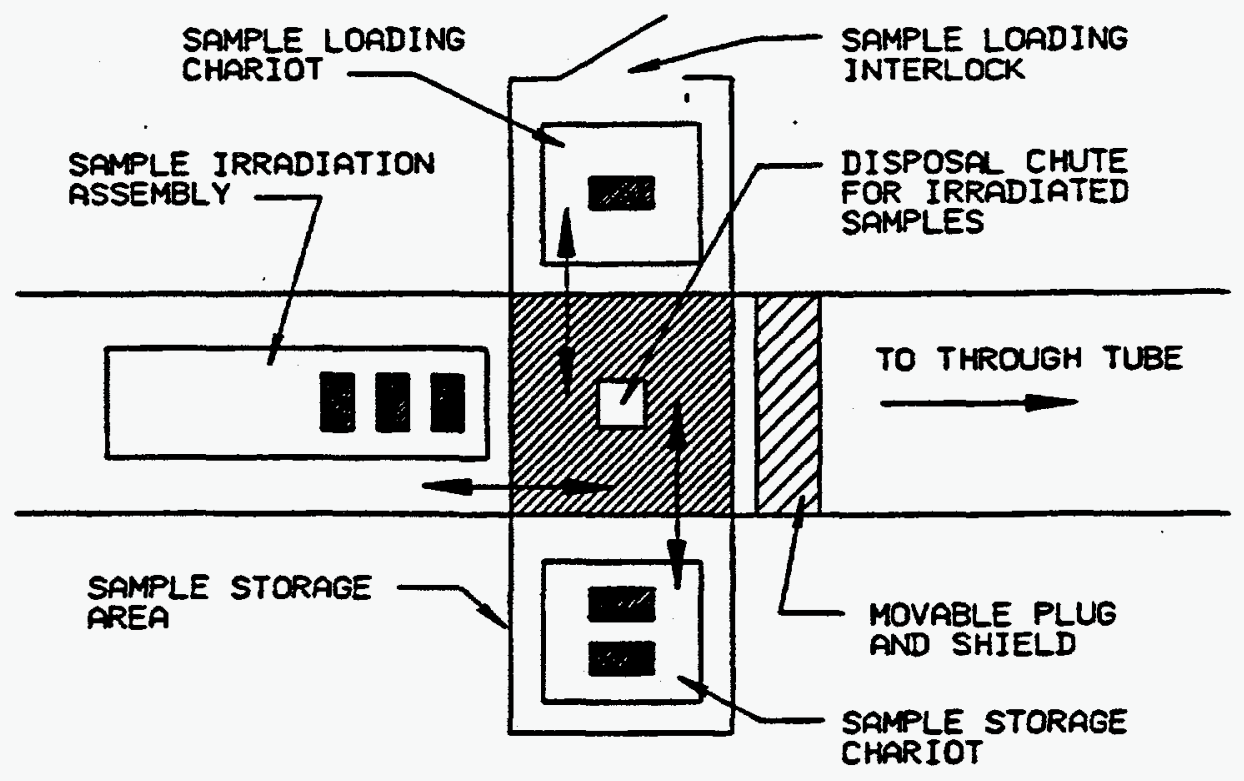

SCHEMATIC PLAN VIEW OF THROUGH TUBE LOADING STATION WITH ALL ELEMENTS WITHDRAWN AND INTERLOCK OPEN FOR INTRODUCTION OF SAMPLE. THE INDICATED ELEMENTS CAN BE BROUGHT INTO THE WORKING AREA FOR VARIOUS SAMPLE MANIPULATIONS.

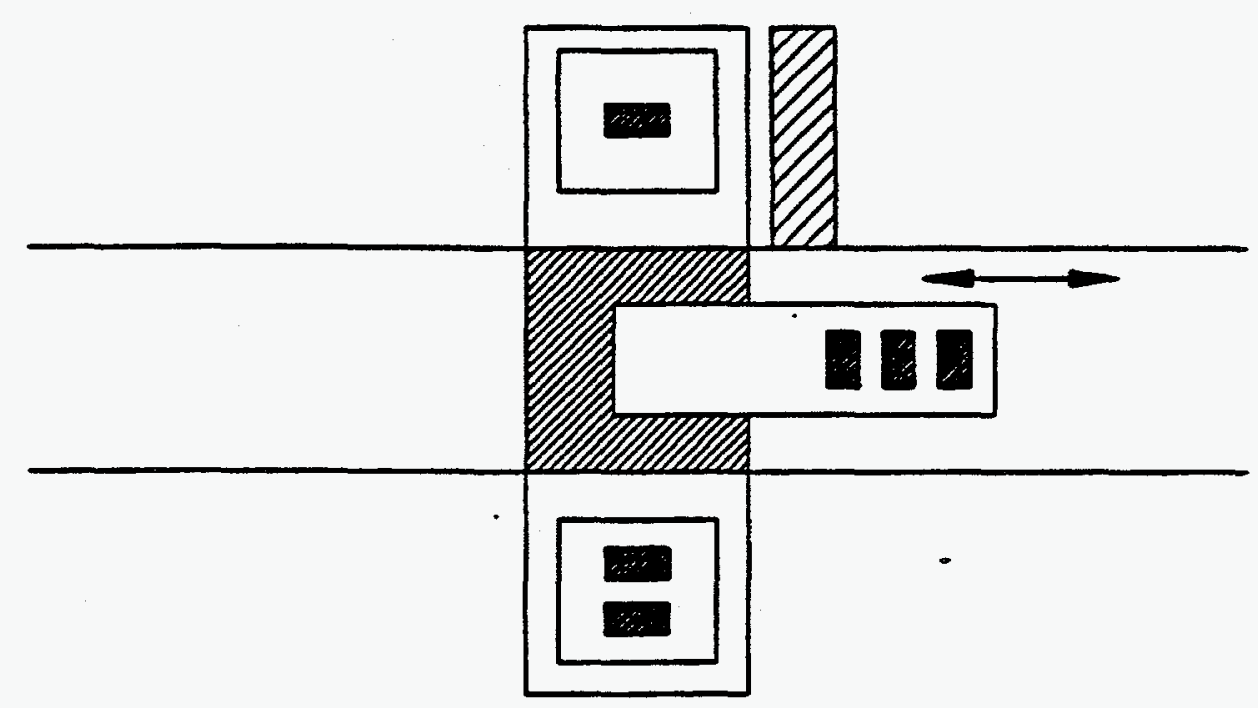

SCHEMATIC PLAN VIEW OF THROUGH TUBE LOADING STATION DURING INSERTION OR WITHDRAWAL OF THE SAMPLE IRRADIATION ASSEMBLY.

Fie 21. Schematic diagram of through tube loading station operations 


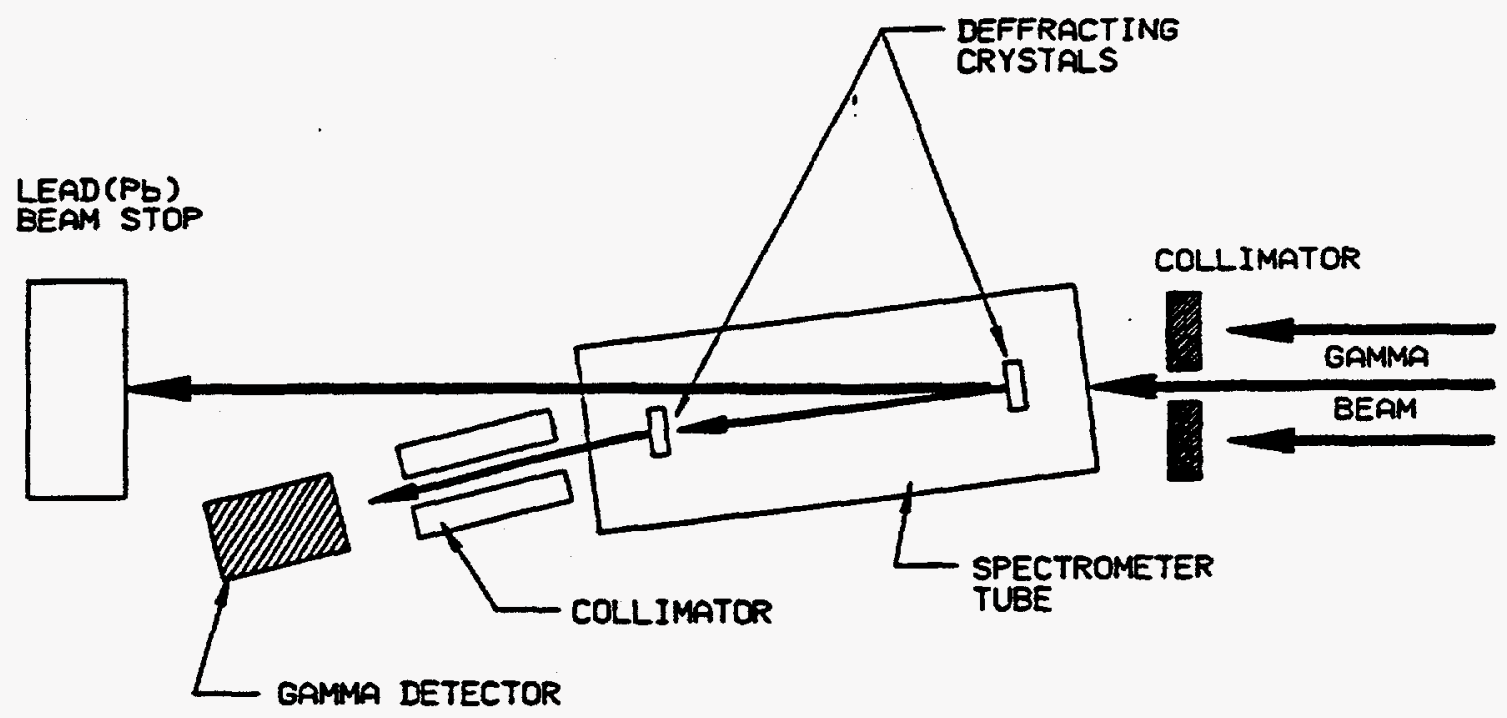

SCHEMATIC PLAN VIEW OF THE CRYSTAL GAMMA-RAY SPECTROMETER IN THE "DOUBLE-CRYSTAL" GEOMETRY.

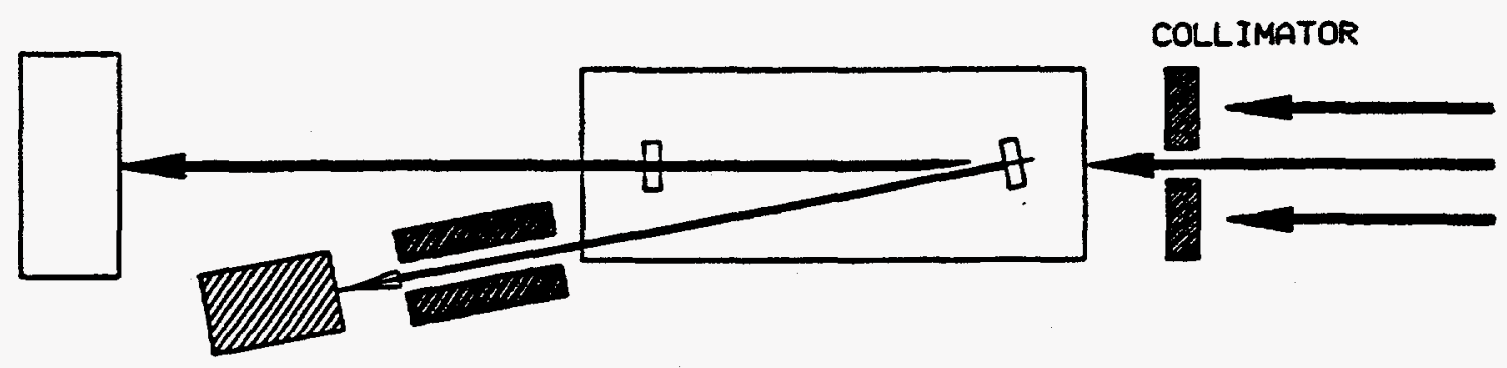

SCHEMATIC PLAN VIEW OF THE CRYSTAL GAMMA-RAY SPECTROMETER IN ITS "SINGLE-CRYSTAL" GEOMETRY.

Fig: 22 Possible goometries of the cystal gamma-ray spectrometer. 


\subsection{Pair formation spectrometer}

When the pair formation spectrometer is in use, the crystal spectrometer will be withdrawn from the $\gamma$-beam axis and extra collimation will be inserted just ahead of the detector. The arrangement of instruments within the enclosure during this operation is shown in Fig. 2.3.

\subsubsection{Polarized Nuclear Physics (WBS 1.4.3.2)}

\subsubsection{Precision $\gamma$-Ray Bolometer (WBS 1.4.3.2.1)}

After optional polarization and filtering, the collimated neutron beam will be allowed to enter the low-temperature area of the dilution refrigerator. The neutron beam will be allowed to impinge only on the region of the cold finger of the refrigerator that contains the sample to be studied. Neutrons that do not interact with the sample will be allowed to exit the low-temperature region, via a rear port, and will then be directed to a remote beam dump. Fig. 2.4 shows this arrangement in schematic form.

The cold finger of the dilution refrigerator will be an extension of the ${ }^{3} \mathrm{He}-{ }^{4} \mathrm{He}$ mixing chambers. Extraction and insertion of such cold fingers will be possible while the refrigerator is operating by means of a top-loading facility, shown schematically in Fig. 2.5.

The necessary pumps and controls for the refrigerator will be contained in a housing adjacent to the cryostat mounting.

\subsubsection{Weak Interaction Physics (WBS 1.4.3.2.2)}

After optional polarization and/or filtering of the neutron beam, it will be directed to a station containing user-supplied equipment. After passage through this equipment, it may be necessary to insert a beam-diagnostic apparatus and a beam dump. (See Fig. 2.6.)

\subsubsection{Parity Violation (WBS 1.4.3.2.3)}

After optional polarization and/or filtering of the neutron beam, it will be directed to a station containing user-supplied equipment. After passage through this equipment, it may be necessary to insert a beam-diagnostic apparatus and a beam dump. (See Fig. 2.6.)

\subsubsection{General Nuclear Physics (WBS 1.4.3.3)}

\subsubsection{Nuclear Structure (WBS 1.4.3.3.1)}

A collimated neutron beam will be directed to an evacuated chamber, where it will interact with a sample. Scattered neutrons will be eliminated using ${ }^{6} \mathrm{Li}$ shielding, and transmitted neutrons will be directed to a remote beam dump. (See Fig. 2.7.)

The emitted nuclear radiation will be detected by an array of detectors of various types, depending on particular experimental considerations.

If charged-particle detectors are to be used, these will be housed within, or coupled directly to, the sample chambers (see Fig. TBD). Gamma-ray and neutron detectors will be mounted in a flexible skeletal framework. (See Fig. 2.8.) 


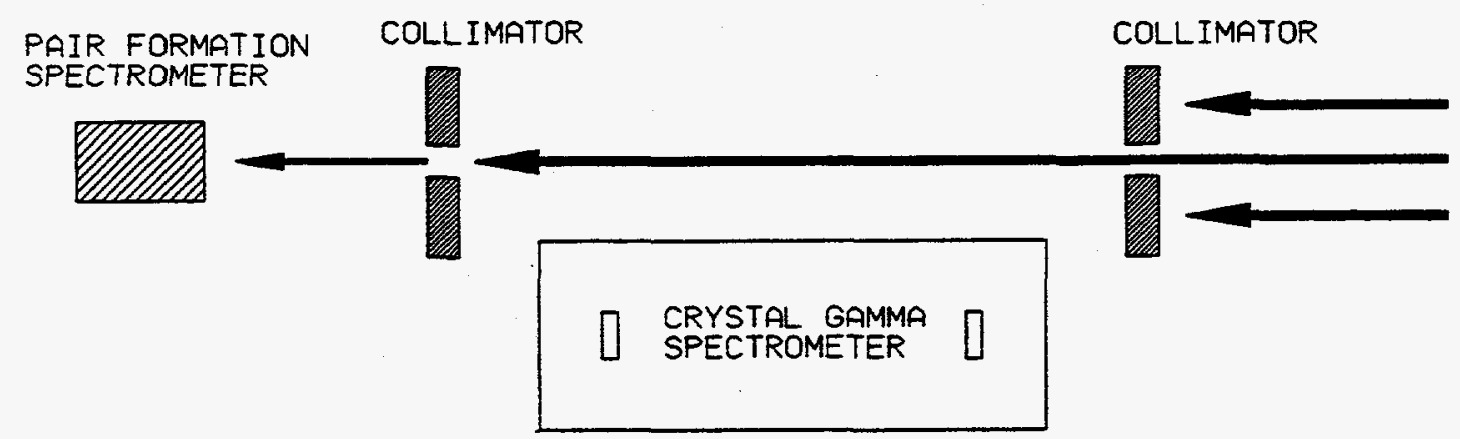

Fig. 23. Schematic plan view of the ultrahigh resolution spectroscopy station when configured for the formation spectrometer. 


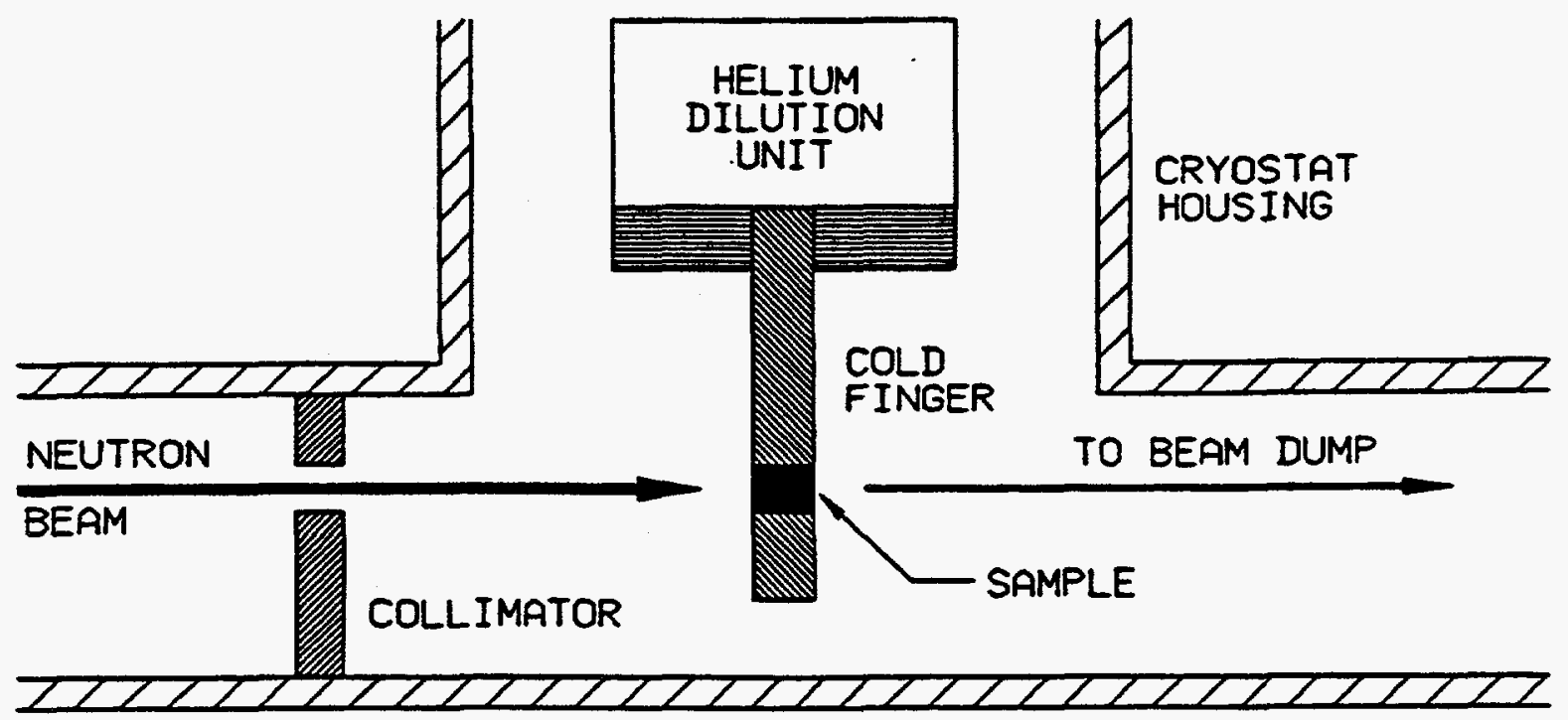

Fig. 24. Schematic side view of rample area in the helium dilution refrigerator. 


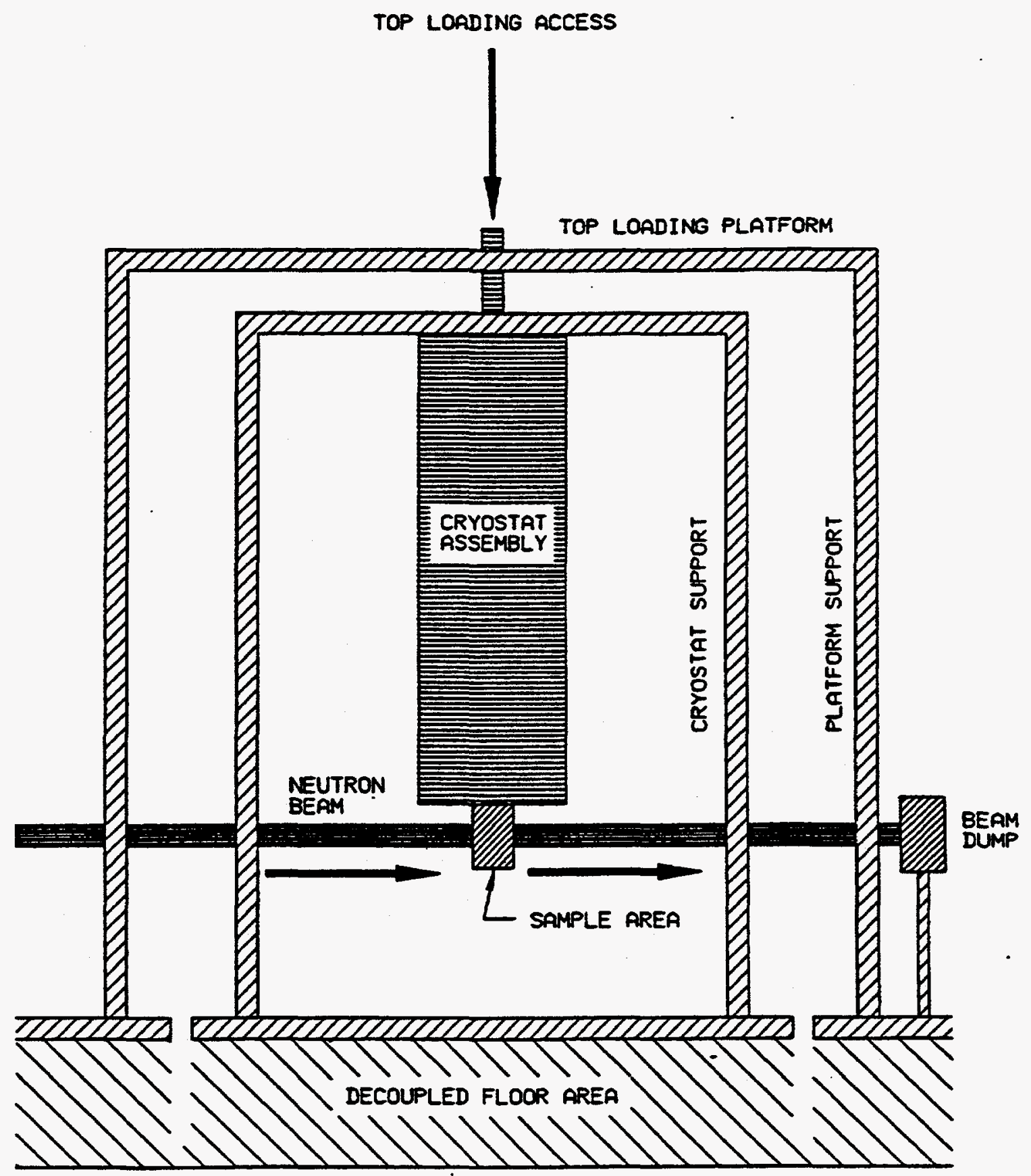

Fig. 25. Sehematic view of the helium dilution refrigerator. 


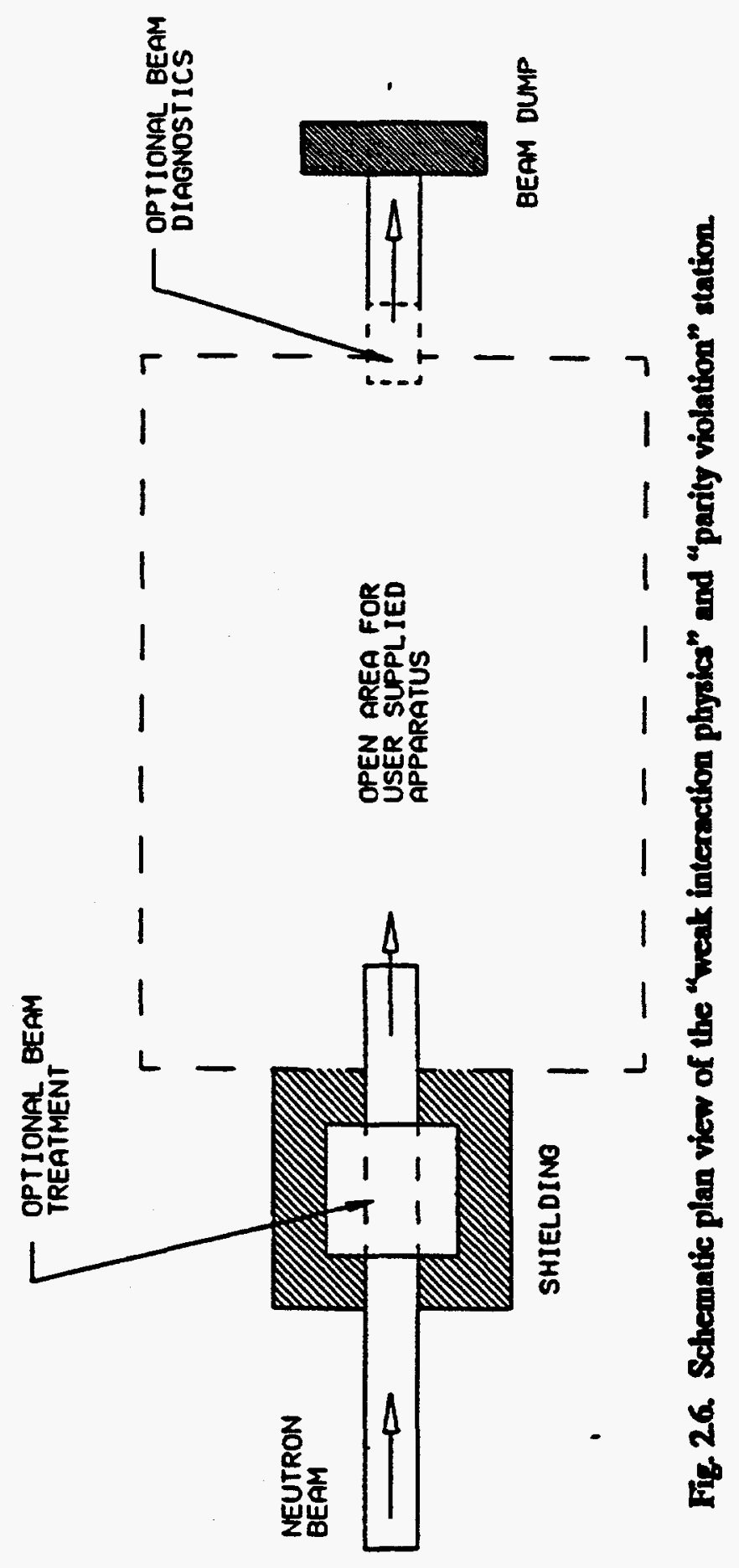




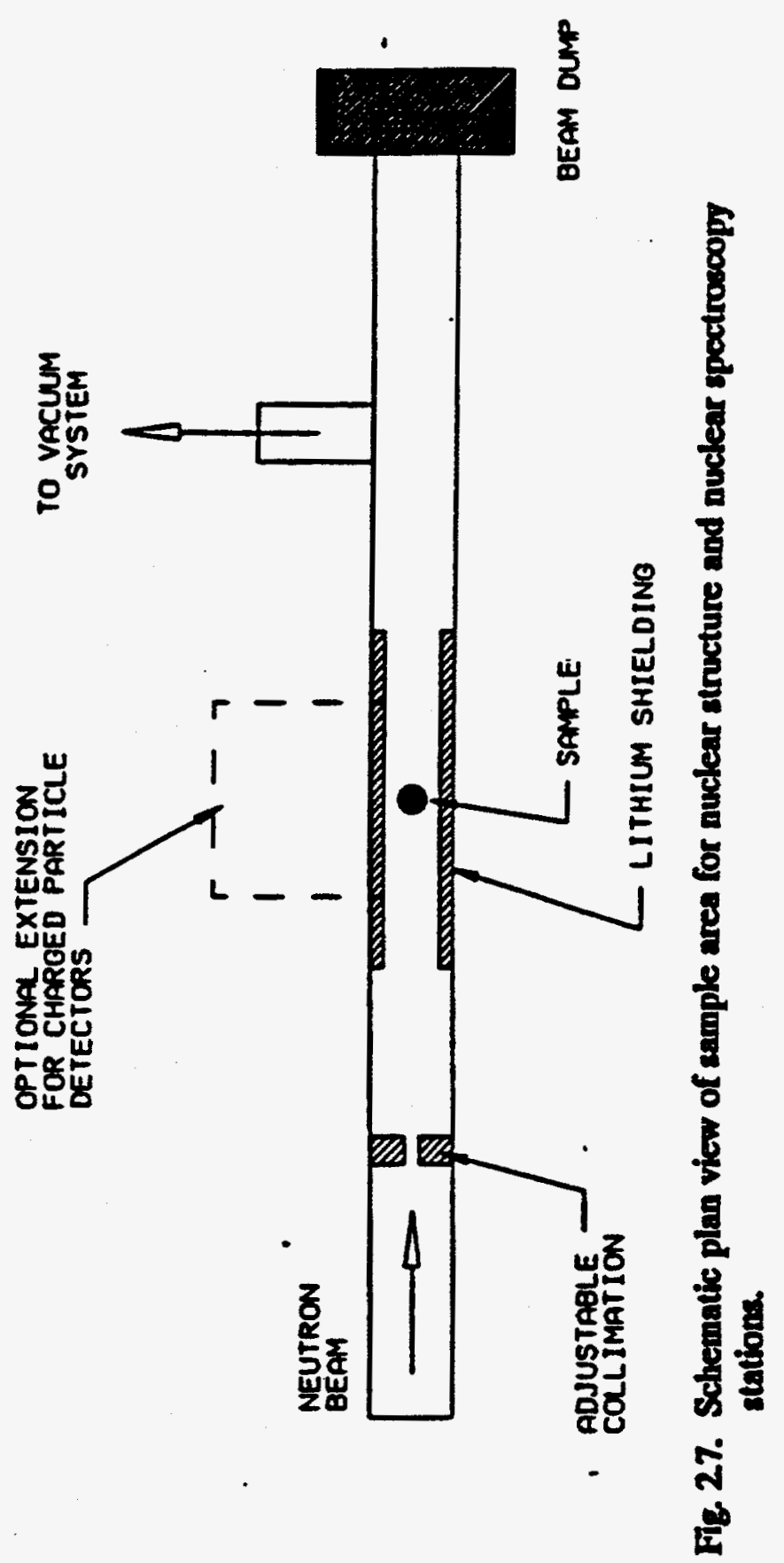

Appendix A, TM-13089 


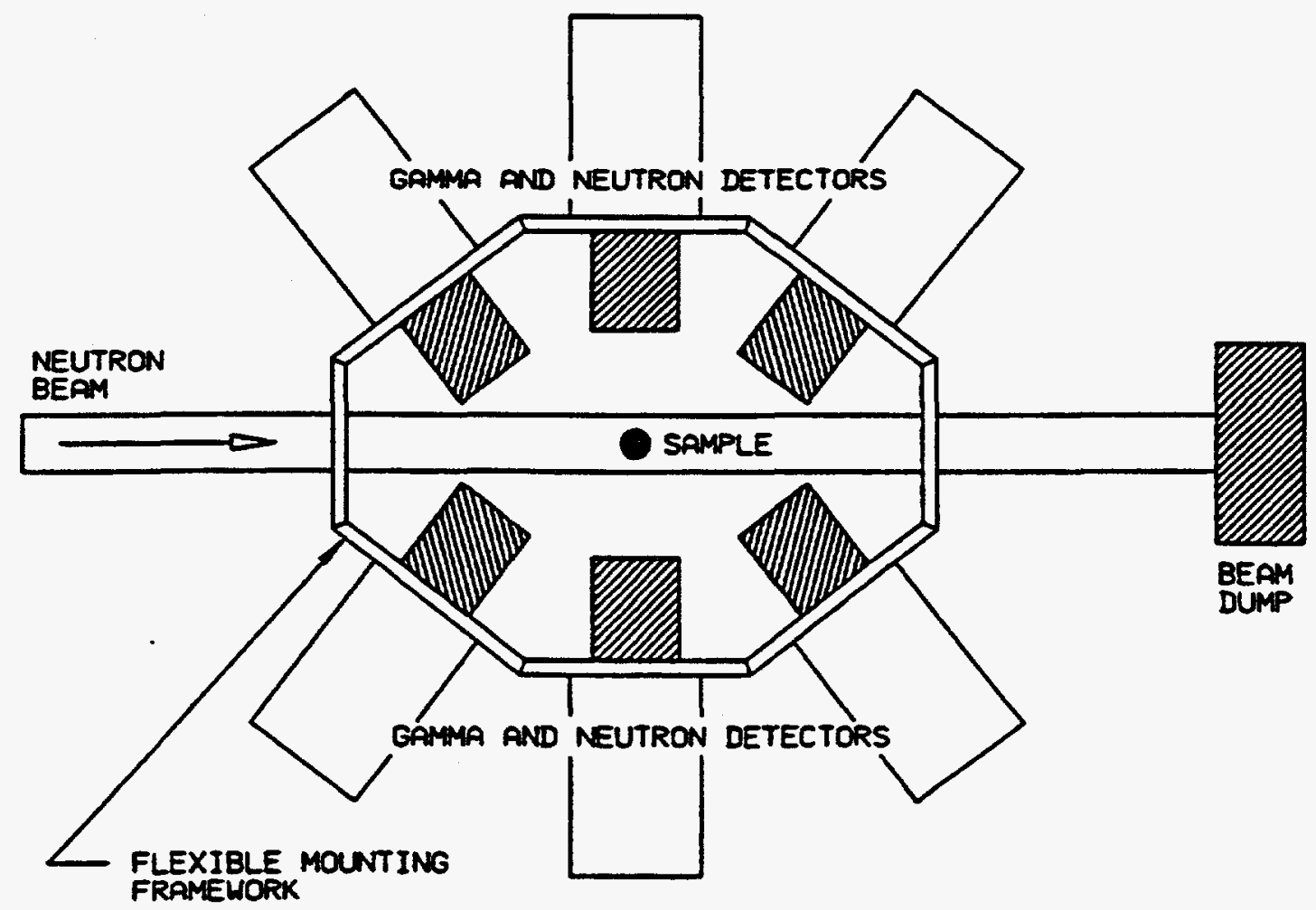

Fig 28 Schematic horizontal section through flerible detector arry.

Fig. 2.8. Schematic horizontal section through flexible detector array. 


\subsubsection{Nuclear Spectroscopy (WBS 1.4.3.3.2)}

A collimated neutron beam will be directed to an evacuated chamber, where it will interact with a sample. Scattered neutrons will be eliminated using ${ }^{6} \mathrm{Li}$ shielding, and transmitted neutrons will be directed to a remote beam dump. (See Fig. 2.7.)

The emitted nuclear radiation will be detected by an array of detectors of various types, depending on particular experimental considerations.

If charged particle detectors are to be used, these will be housed within, or coupled directly to, the sample chambers. Gamma-ray and neutron detectors will be mounted in a flexible skeletal framework. (See Fig. 2.8.)

\subsubsection{Isotope Separation On-Line (WBS 1.4.3.3.3)}

\subsection{Ion source and beam transport}

The ion source arrangement will be introduced into a neutron beam via a shielded cask. Within the ion source, neutrons will interact with milligram quantities of fissile material and the resulting ionized fission fragments will be allowed to recoil in the direction of the mass separator. In between, the ion beam will be controlled and steered by a system of electromagnetic lenses.

\subsection{Isotope separation}

The ion beam will pass between the poles of a large electromagnet and then between the plates of a large condensor arrangement.

By adjusting the electromagnetic fields in these regions, different ion masses can be guided to a well-defined exit port, which will interface to a measurement station. (See Figs. 2.9 and 2.10.)

\subsection{Measurement station}

The measurement station will be configured in one of several ways:

- $\quad$ direct measurement of nuclear radiation by a flexible array of detectors (Sects. 2.4.3.1 and 2.4.3.2 and Fig. 2.8),

- deposition of ions onto a tape transport system for measurement of subsequent nuclear radiation off-line by a flexible array of detectors, direct injection into a heavy-ion accelerator for the production of neutron-rich radioactive beams, and

implantation of ions into a host for later study of solid state effects.

\subsubsection{Neutron Interferometer (WBS 1.4.2.8.1)}

Figure 2.11 is a schematic drawing of the neutron interferometer. A double-crystal monochromator system is used to extract a monochromatic neutron beam from the neutron guide. This beam is then directed to the interferometer, which is housed in a controlled environment. 


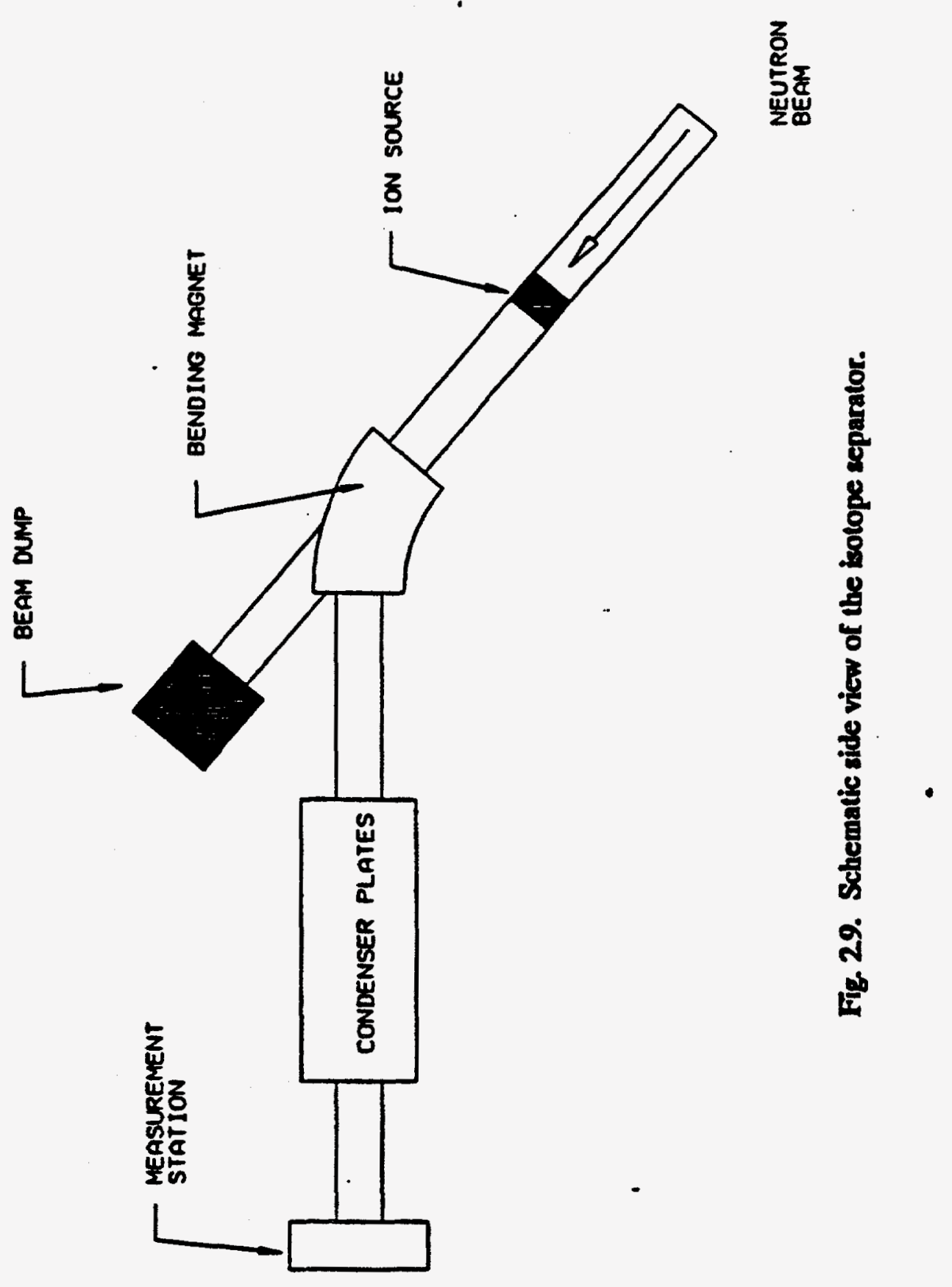




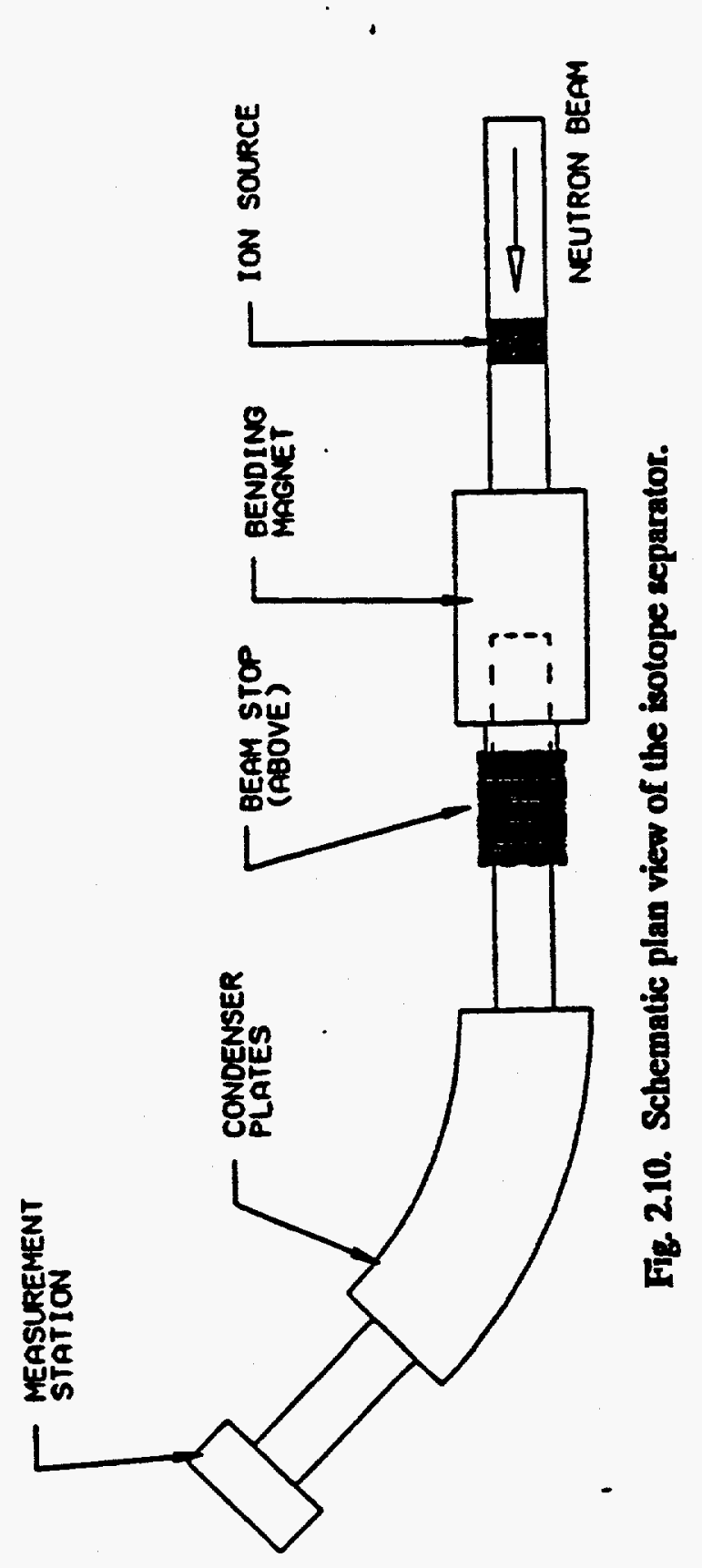




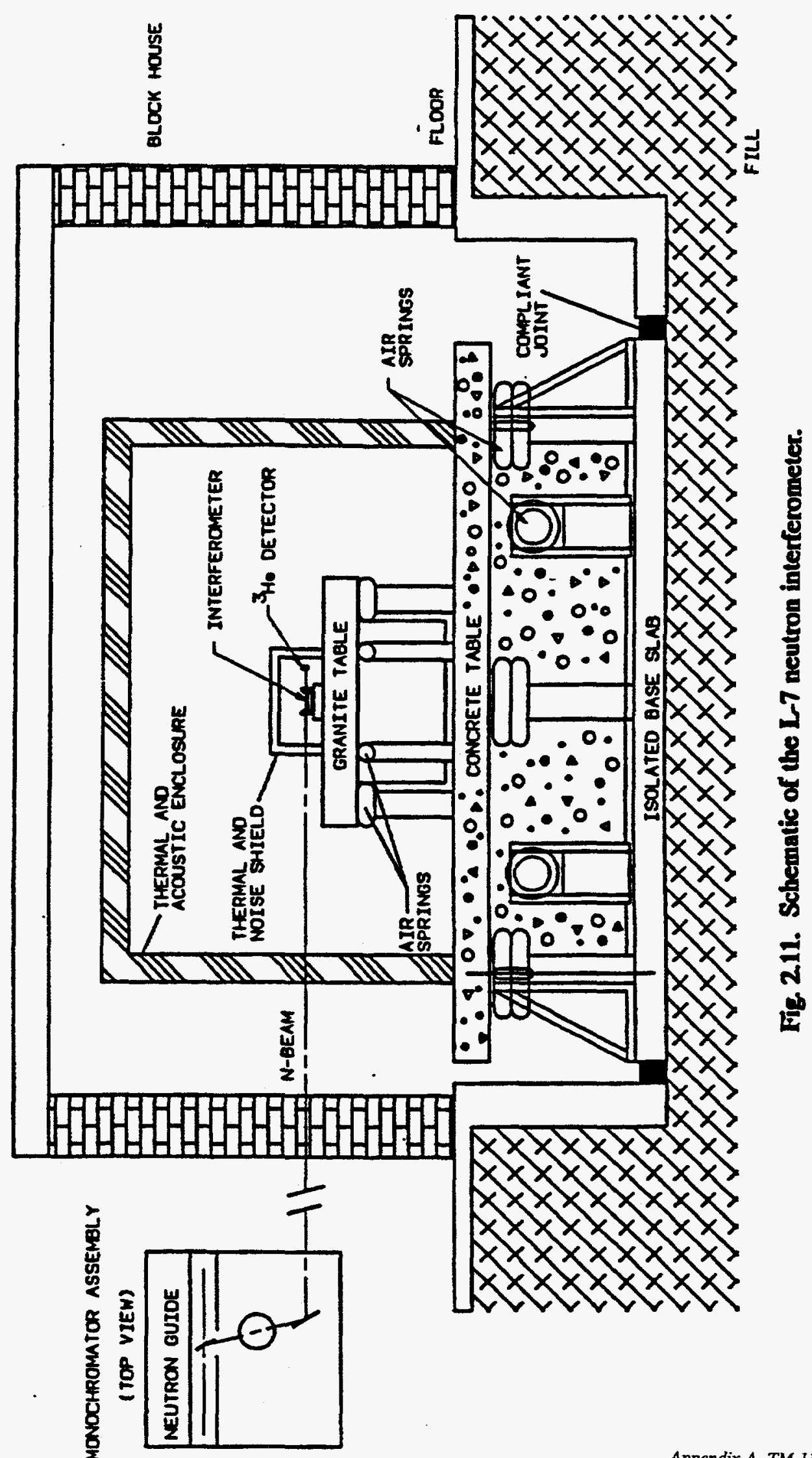




\subsection{COMPONENT DESIGN DESCRIPTIONS}

\subsubsection{Thermal Nuclear Physics (WBS 1.4.3.1)}

\subsubsection{Through Tube Loading Station (WBS 1.4.3.1.1)}

The through tube loading station will consist of several chambers located around a central work area. These chambers will house various sample-holding assemblies, which can be introduced into the work area when desired. One side of the loading station will interface to the tangential through tube (WBS 1.4.1.2.2) to allow the introduction and withdrawal of the sample-irradiation assembly. When such operations are not taking place, this interface will be plugged and shielded. All chambers will operate in a common inert atmosphere.

With all the sample-holding assemblies withdrawn, access will be possible to the floor of the work area, in which will be located an interface to a chute for the disposal of previously irradiated, active samples.

The following sections contain details of the various sample-holding assemblies that will have access to the work area.

\subsection{Sample-loading chariot}

After preparation, samples will be introduced into the loading station via an external interface system and placed into the sample-loading chariot. This chariot is then moved into the work area so that the samples can be accessed by the remote-handling devices. After a sample has been transferred, the chariot is withdrawn from the work area.

The sample-loading chariot will accommodate samples of the different designs required by the various spectrometer configurations. In all cases sample suspension and manipulation will be achieved using the same basic mechanism. Occasionally the sample-loading chariot will also be used to remove activated samples from the loading station for external study. The procedure for such operation will be a simple reversal of the sample-loading procedure.

\subsection{Sample-storage chariot}

In the case that a previously irradiated sample must be stored for future use, it will be placed in the sample-storage chariot. Similar in design to the sample-loading chariot, it will hold several different samples (of varying design) in a side chamber next to the work area. When storing (or accessing a previously stored) samples, this chariot will be introduced into the central work area.

\subsection{Sample-irradiation assembly}

The sample-irradiation assembly will consist of a hollow graphite cylinder that can be introduced into the tangential through tube. In the upper surface of the front end, there will be spaces in which samples of varying design can be suspended. When samples are to be loaded or unloaded from this assembly, they will be withdrawn from the through tube and into the work area. When other sample manipulations are to be carried out, this assembly will be further withdrawn to a shielded chamber behind the working area. 
To minimize thermal shocks, the insertion/withdrawal of the assembly to/from the through tube will be accomplished over several minutes. Only during this time will be plug/shield at the loading station/through-tube interface be removed. The final location of the assembly within the through tube will be variable to allow the sample to interact with different neutron fluxes. Once in place within the through tube, optional orientation of one sample position will be available.

\subsection{Work area}

All sample manipulations will take place within the work area. All sample designs will feature a common suspension/manipulation assembly such that they can all be controlled with the same remote access device. This device will consist of a remotely controlled grasping hand, which can descend into the work area from a vertical chamber above. All sample manipulations will be monitored externally with TV equipment.

\subsubsection{Ultrahigh Resolution $\gamma$-Ray Spectroscopy (WBS 1.4.3.1.2)}

Gamma rays from samples introduced into the tangential through tube will be studied at this station. To provide maximum flexibility the $\gamma$ beam will first pass through a variable $\gamma$ collimator before reaching the spectrometers. This collimator will be adjustable in both the vertical and horizontal directions in both the absolute and differential modes. In addition, shielding will be provided to prevent the scattering of stray neutrons into the spectroscopy station.

\subsection{Environmentally controlled enclosure}

Because the $\gamma$ spectrometers are very sensitive to small environmental changes, they will be housed in a sealed enclosure in which the atmosphere will be closely monitored and controlled. The temperature will be controlled by a servoregulated combination of heating and cooling elements responding to temperature probes at various locations in the enclosure and, particularly, on the spectrometers themselves. In addition, the floor beneath the crystal spectrometer table will be isolated from external vibrations by using both active and passive damping systems.

All spectrometer functions will be controlled from an external computer system and monitored with a remote TV system. The various spectrometer elements within the enclosure are discussed in the following paragraphs.

\subsection{Crystal spectrometer}

The crystal $\gamma$-ray spectrometer will be supported on a large, vibration-insulated plate. The upper surface of this plate will be smooth to allow spectrometer components to move on air-pads. The table carrying the diffracting crystals and associated interferometer optics will travel on air-pads on this large pate.

The diffracting crystals will be mounted on rotating vertical axes, which will be adjustable to allow for alignment correction of the diffracting planes. The angle of rotation will be controlled and monitored by using a laser-based interferometer system that will measure relative and absolute movements of the axes via selected optical paths. Positioning of the axes will be accomplished by using high-precision stepping motors and controllers. The lateral and rotational positions of the spectrometer table itself will also be accomplished with stepping motors. The diffracting crystals 
themselves will be cut such that possible distortion of the diffracting plane resulting from the stress of the mechanical mounting is eliminated.

After diffraction through one or two crystals (depending on the desired spectrometer geometry), the selected $\gamma$ beam will pass through a long collimator to reach a $\gamma$ detector. The positions of the latter will again be controlled by stepping motors to coincide with the diffracted beam. The $\gamma$ detector itself will be a standard hyperpure germanium (HPGe) detector (see Sect. 2.5.3) but will be heavily shielded from both external background and the very intense direct (i.e., nondiffracted) $\gamma$ beam. It is also essential that other instruments in the region of the spectrometer keep their background generation to the absolute minimum achievable.

\subsection{Pair formation spectrometer}

The principle of operation of the pair formation spectrometer has been discussed in Sect. 2.2.1.2.2. When in operation it will use the same sample-irradiation assembly (and $\gamma$-beam collimation) as the crystal spectrometer. However, the crystal spectrometer table will be laterally displaced from the beam axis and extra, adjustable collimation will be placed in front of the pair formation spectrometer.

The spectrometer will consist of a small central HPGe detector surrounded by large-volume scintillation detectors of limited energy resolution. The detector shielding will be arranged such that the scintillation detectors view only the central HPGe detector and do not see the incoming direct $\gamma$ beam. The whole detector assembly will be mounted on a table, the height and lateral position of which will be adjustable. When not in use, the whole spectrometer will be laterally displaced from the direct beam axis.

\subsubsection{Polarized Nuclear Physics (WBS 1.4.3.2)}

\subsubsection{Precision $\gamma$-Ray Bolometer (WBS 1.4.3.2.1)}

The main component of the bolometer will be a commercially purchased helium dilution refrigerator. The specifications will stipulate a sample area located on a copper extension (cold finger) from the low-temperature cryogenic stage such that the sample can be placed directly in the neutron beam. Pretreatment of this beam may include polarization and chopping. In addition, precise collimation is necessary to ensure that neutrons do not activate material in the immediate environment of the sample. For this reason also, the area of the cold finger immediately behind the sample should be as thin as possible.

Exchange of samples will be achieved by using a top-loading technique whereby samples are inserted and withdrawn along a channel that passes down the vertical axis of the cryostat. Because this involves the insertion of a long syphon, a stable platform will be provided at the top of the cryostat to allow manual control of this operation.

The neutron guide will be coupled directly to the cryostat both in front of and behind the sample position. To ensure the integrity of the cryostat vacuum system, thin beryllium windows may be used at these points. Also in the sample region (but not in the direct neutron beam), there will be a pair of superconducting Helmholtz coils, which will be used to generate the large magnetic fields required for nuclear orientation. Care will be taken to shield all such sensitive components from the effects of stray neutron scattering. 
Detection systems will consist of $\gamma$-ray and charged-particle detectors from the general pool. All the control systems for the dilution refrigerator will be housed in an adjacent cabinet.

\subsubsection{Weak Interaction Physics (WBS 1.4.3.2.2)}

This station will be left open for user-supplied equipment. Pretreatment of the neutron beam may include polarization and chopping. After passage through the experimental station, a position for the insertion of beam diagnostic equipment will be provided in front of a remote beam dump.

\subsubsection{Parity Violation (WBS 1.4.3.2.3)}

This station will be left open for user-supplied equipment. Pretreatment of the neutron beam may include polarization and chopping. After passage through the experimental station, a position for the insertion of beam diagnostic equipment will be provided in front of a remote beam dump.

Because certain experiments may involve the use of multiliter quantities of liquid hydrogen, this station will be located in a separate area of the facility. The structure of this location will be designed to minimize the effects of a worst-case scenario involving a leak of hydrogen and subsequent explosion. All possible measures will be taken to ensure early detection of such leaks.

\subsubsection{General Nuclear Physics (WBS 1.4.3.3)}

\subsubsection{General Detector Pool}

The various general nuclear physics stations will all use similar detector configurations taken from a general pool of detectors discussed in the following sections. A pool of associated electronics will also be maintained.

\subsection{Gamma-ray detectors}

Different types of $\gamma$-ray detectors will be used, depending on the experiment configuration desired.

2.5.3.1.1.1 Germanium detectors. The standard device used for $\gamma$-ray detection consists of a large biased HPGe diode. When a $\gamma$ ray interacts with this diode, the energy deposited can be detected as a current produced through the diode. Dedicated electronic modules integrate this current to produce pulses, the height of which are proportional to the energy deposited. To minimize interference from electronic noise, HPGe detectors are operated at liquid nitrogen temperatures attained by using a cold finger inserted into a dewar, which forms an integral part of the detector assembly.

Such detectors are available commercially and have relative efficiencies [with respect to a $76.2 \mathrm{~mm}$ ( $3 \mathrm{in}$.) by $76.2 \mathrm{~mm}$ ( $3 \mathrm{in}$.) NaI crystal] of up to $120 \%$. At least ten of these detectors will be needed for the general pool. To facilitate the flexible mounting of different geometrical arrays of these detectors, small dewars are essential. In this case these dewars must be filled every few hours; thus, automatic filling systems will be used. To minimize the effects of neutron damage, n-type HPGe detectors will be used.

2.5.3.1.1.2 Compton suppression shields. When a $\gamma$ ray interacts in a HPGe detector, it may leave only part of its energy within the germanium crystal, with the rest being scattered out (Compton 
effect). In such cases the detector does not record the full energy of the $\gamma$ ray, giving rise to an almost continuous distribution of energies that provide only limited information. Such signals can swamp the full-energy signals from weak, lower-energy $\gamma$ rays.

To counter this effect HPGe detectors are often enclosed in shields made from scintillator material. Although the energy resolution of such shields is vastly inferior to germanium detectors, they can still be effectively used to reject any signals in the HPGe detector that occur simultaneously with signals in the scintillator as a result of Compton scattering. The best choice of material for such scintillators is bismuth germanate (BGO) because it offers the best stopping power for $\gamma$ rays in the least volume.

The general detector pool will have at least six of these Compton suppression shields. Their geometry will be such that the HPGe/shield systems can be closely packed around the measurement stations. Such systems can be purchased commercially to user-supplied specifications.

2.5.3.1.1.3 Fast timing detectors. Although HPGe detectors provide superior energy resolution, they are of limited use when optimum timing is required. By using small scintillation crystals that combine fast response time and limited energy resolution, it is possible to measure the lifetime of excited nuclear states when they lie above a few picoseconds. The choice of crystal for this application is barium fluoride, coupled to a fast photomultiplier. Organic scintillators may also be needed when charged-particle radiation is involved. The general detector pool will have three each of such detector systems. The components are commercially available, and assembly is trivial.

\subsection{Particle detectors}

2.5.3.1.2.1 Sector magnet electron spectrometer. In certain experiments it will be necessary to measure, with good resolution, the energy of $\beta$ particles and conversion electrons coming from nuclear decay. In addition, these measurements will often be performed in coincidence with signals from $\gamma$-ray detectors. The instrument of choice in this case is a sector magnet spectrometer, which combines good resolution $(0.5 \%)$ with low background and the ability to use multistrip target techniques to increase efficiency.

Such a system, using magnetic fields to guide electrons from the target to the detector, eliminates interference from $\gamma$ radiation (the direct path of which is blocked).

\subsection{Charged-particle detectors}

The general pool will also have several silicon surface barrier detectors for studying chargedparticle emission during neutron-induced reactions. Such detectors are commercially available in configurations suitable for studying electrons, protons, and $\alpha$ particles.

\subsection{Neutron detectors}

In some nuclear decays fast neutrons are emitted. Commercially available detectors for such neutrons will also be in the general detector pool. 


\subsubsection{Nuclear Structure (WBS 1.4.3.3.1)}

The sample chamber for the nuclear structure station will be directly coupled to the incoming neutron guide. Samples, in graphite containers, will be placed in the neutron beam, and subsequent nuclear radiation will be detected with systems selected from the general detector pool (see

Sect. 2.5.3.1). The particular detector configuration will be dictated by the parameters of individual experiments. Where appropriate, a flexible skeletal framework will be used to mount the detectors.

In the section of neutron guide ahead of the sample chamber, continuously variable neutron collimation with optional beam polarization and/or chopping will occur. The sample position itself also will be adjustable within the sample chamber.

The sample chamber will be of the minimum size that can accommodate the sample, so that the radiation detectors can be placed as closely as possible to the sample. The inner surfaces of the sample chamber will be lined with neutron-absorbing materials to minimize detector damage. In cases in which charged-particle detectors must be placed within the same vacuum as the sample, extensions to the sample chamber will be used.

\subsubsection{Nuclear Spectroscopy (WBS 1.4.3.3.2)}

The sample chamber for the nuclear structure station will be coupled directly to the incoming neutron guide. Samples, in graphite containers, will be placed in the neutron beam, and subsequent nuclear radiation will be detected with systems selected form the general detector pool (see Sect. 2.5.3.1). The particular detector configuration will be dictated by the parameters of individual experiments. Where appropriate, a flexible skeletal framework will be used to mount the detectors.

In the section of neutron guide before the sample chamber, continuously variable neutron collimation with optional beam polarization and/or chopping will occur. The sample position itself will also be adjustable within the sample chamber.

The sample chamber will be of the minimum size that can accommodate the sample, so that the radiation detectors can be placed as closely as possible to the sample. The inner surfaces of the sample chamber will be lined with neutron-absorbing materials to minimize detector damage. In cases in which charged-particle detectors must be placed within the same vacuum as the sample, extensions to the sample chamber will be used.

\subsubsection{Isotope Separation On-Line}

\subsection{Ion source}

The ion source assembly will consist of a plug to be introduced into the biological shielding of the reactor. This plug will contain milligram quantities of fissile material in a supporting matrix. Neutrons coming up the inclined neutron beam will interact with this material and produce isotopic fission fragments. The ion source assembly also will contain a means of ionizing these fragments. (The exact technique will depend on the chemical species to be extracted.) The ionized fragments then will be guided (using magnetic lens elements) to the entrance of the separation stage. Several such ionsource plugs will be available for use with different fissile materials and configured for different chemical species. 


\subsection{Isotope separation}

The ionized fission fragments will pass between the flat poles of a large electromagnet, which drives them into a circular path, the radius of which is determined by the mass, velocity, and ionic charge of the fragments and the magnitude of the applied magnetic field. After this the ion beam will enter between the curved plates of a large condenser. An electric field is applied between these plates.

The combination of electric and magnetic fields can be varied to produce, at the exit of the separator stage, a beam of fission fragments characterized by a unique ionic charge-to-mass ratio. This beam will then be guided to the measurement station.

\subsection{Measurement station}

The sample chamber for the measurement station will be directly coupled to the separator. The incoming ions will be collected at a fixed point where subsequent nuclear radiation may be detected with systems selected from the general detector pool (see Sect. 2.5.3.1). The particular detector configuration will be dictated by the individual experiment parameters. Where appropriate, a flexible skeletal framework will be used to mount the detectors.

In some cases the ions will be collected on a tape transport system to be carried, periodically, to a remote measuring position. The collection and transport times will be dictated by considerations of individual experiments. For ion implantation studies the sample will be placed directly at the collection position and later removed for off-line study.

An option also will be available to couple the collection station directly to a further beam transport system for use in the generation of neutron-rich RIBs.

\subsubsection{Neutron Interferometer (WBS 1.4.2.8.1)}

\subsubsection{Double-Crystal Monochromator}

The variable-energy monochromatic neutron beam will be provided by a double-crystal assembly. The range of diffraction angles will be from $40^{\circ}$ to $110^{\circ}$, providing neutron wavelengths of 0.13 to $0.31 \mathrm{~nm}(1.3$ to $3.1 \AA$ ) with silicon (220) crystals and 0.23 to $0.55 \mathrm{~nm}$ ( 2.3 to $5.5 \AA$ ) with PG (002) crystals. To reduce attenuation caused by air scattering, the beam paths will be constructed by using telescoping aluminum tubing filled will gaseous helium and silicon crystal end windows. The monochromator crystals will encompass the full cross-sectional area of the neutron beam. The monochromator elements will be mounted on rotary tables and controlled by precision rack systems.

\subsubsection{Isolated Enclosure}

The thermal isolation of the interferometer will be accomplished by using three stages, or nested systems.

1. Construction of a large blockhouse, the internal temperature of which will be controlled by using a combination of heating and cooling elements.

2. Mounting the interferometer on a large (mass of about 3 tons) granite or cast-iron plate, the temperature of which will be maintained with a combination of heating and cooling elements controlled by servosystems. 
3. Positioning a small isothermal box around the interferometer proper, the temperature of which will be controlled using a servobased heater/cooler system.

To minimize vibrational and seismic disturbances of the interferometer system, a three-stage nested isolation system will be used.

1. The building must be designed so that the interferometer position rests on an independent foundation coupled to the rest of the building by compliant joints only.

2. A pneumatically supported concrete slab $5.5 \mathrm{~m} \times 4.3 \mathrm{~m} \times 0.9 \mathrm{~m}(18 \mathrm{ft} \times 14 \mathrm{ft} \times 3 \mathrm{ft})$ of approximately $22.7 \mathrm{Mg}$ (25 tons) will significantly reduce high-frequency vibrations. A smaller [2.7 Mg (3 tons)] slab, also pneumatically supported, will be positioned on top of the larger slab.

3. Active damping elements will be used to lock the upper table to an inertial proof mass mounted on the table. This proof mass will consist of a commercially available long-period seismometer.

\subsubsection{Neutron Interferometer}

The interferometer itself will be constructed of perfect silicon crystals. These will be mounted on the table and controlled by standard techniques. After passage through the crystal, neutrons will be detected by using a position-sensitive detector with $1-\mathrm{mm}(0.04 \mathrm{in}$.) resolution.

\subsection{INSTRUMENTATION AND CONTROL}

\subsubsection{Thermal Nuclear Physics (WBS 1.4.3.1)}

\subsubsection{Through Tube Loading Station (WBS 1.4.3.1.1)}

All functions of the through tube loading station will be controlled from an adjacent location. Three distinct groups of functions to be controlled from here can be identified.

1. The remote sample handling in the work area will be performed with a robot arm. This arm will pick up or insert individual samples from the various sample-holding assemblies that can be moved into the work area. In addition to the vertical degree of freedom, limited lateral and rotational motions also will be possible.

2. The sample irradiation assembly will be controlled during insertion and withdrawal from the through tube. During such operations, the through tube plug/radiation-shield will be withdrawn. Once in place, the rotation of the central sample position also will be possible.

3. The control of the helium gas circulation system will be via a network of remotely controlled valves. Pressure gauges also will be located centrally to ensure compatibility of different areas before physical connection. In case of failure of the remote system, some manual control of certain critical valves will be necessary. Gas exiting from the through tube will be monitored for increased radioactivity.

Such manipulations will be carried out through a computer interface and monitored, where appropriate, via a TV monitor system. The appropriate software and hardware will contain fail-safe mechanisms to prevent improper operation. It also will be necessary for certain of these functions to 
be monitored in the reactor control room to ensure early detection of possible problems resulting from sample disintegration or loss of integrity of the internal atmosphere.

\subsubsection{Ultrahigh Resolution $\gamma$ Spectroscopy (WBS 1.4.3.1.2)}

The exact positioning of the elements of the crystal $\gamma$-ray spectroscope will be controlled by using a computer and standard interface techniques. Visual monitoring will be accomplished by using remote TV systems. The same system will be used to control the pair-formation spectrometer and to perform all data acquisition tasks.

\subsubsection{Polarized Nuclear Physics (WBS 1.4.3.2)}

\subsubsection{Precision $\gamma$-Ray Bolometer (WBS 1.4.3.2.1)}

The control system for the dilution refrigerator will be housed in an adjacent cabinet. System performance will be monitored by periodic noting of system parameters essential to correct functioning. Data acquisition will be accomplished using a separate computer and interface.

\subsubsection{Weak Interaction Physics (WBS 1.4.3.2.2)}

In-house control is limited to beam diagnostic tasks.

\subsubsection{Parity Violation (WBS 1.4.3.2.3)}

In addition to standard beam diagnostics, this area will be monitored for leaks of hydrogen, when in use.

\subsubsection{General Nuclear Physics (WBS 1.4.3.3)}

\subsubsection{Nuclear Structure (WBS 1.4.3.3.1)}

Both neutron beam collimation and sample position will be controlled manually. Data acquisition will be accomplished by using a separate computer and interface.

\subsubsection{Nuclear Spectroscopy (WBS 1.4.3.3.2)}

Both neutron beam collimation and sample position will be controlled manually. Data acquisition will be accomplished by using a separate computer and interface.

\subsubsection{Isotope Separation On-Line (WBS 1.4.3.3.3)}

The magnetic and electric beam steering elements will be controlled by a dedicated computer and appropriate interface. Ion source performance will be indicated by experimental count rates. A separate computer system will control the optional tape transport mechanism and the data acquisition cycles. 


\subsubsection{Neutron Interferometer (WBS 1.4.2.8.1)}

The thermal and vibrational control systems will be controlled by dedicated electronics systems. The mechanical positioning of the monochromator and interferometer elements will be performed by a dedicated computer and interface system, which will also be responsible for data acquisition.

\subsection{SYSTEM INTERFACES}

As a standard all computer systems will have access to the site network. In addition all stations will be connections to the gas, vacuum, and other systems detailed in Sect. 1.2.7. Specific other interfaces are given in the following sections.

\subsubsection{Through Tube Loading Station (WBS 1.4.3.1.1)}

The environmental system of the loading station will interface to that of the through tube itself and, hence, to the "in-house" helium-recovery circuit. During sample manipulations close coordination with overall reactor control and radioprotection is necessary. At all times the main control room must have access to parameters that could indicate possible sample disintegration and/or loss of integrity of the internal atmosphere in the through tube.

\subsubsection{Parity Violation (WBS 1.4.3.2.3)}

When liquid hydrogen is in use, this station will interface directly to alarm and safety systems in the event of a detected leak.

\subsubsection{Isotope Separation On-Line (WBS 1.4.3.3.3)}

During insertion or removal of the ion source "plug" from the reactor shielding, close coordination with overall reactor control and radioprotection is necessary. 
Appendix A

STANDARDS, CODES AND GUIDES

TBD [HOLD] 

Appendix B

PARAMETERS LIST

TBD

B-1

Appendix A, TM-13089 


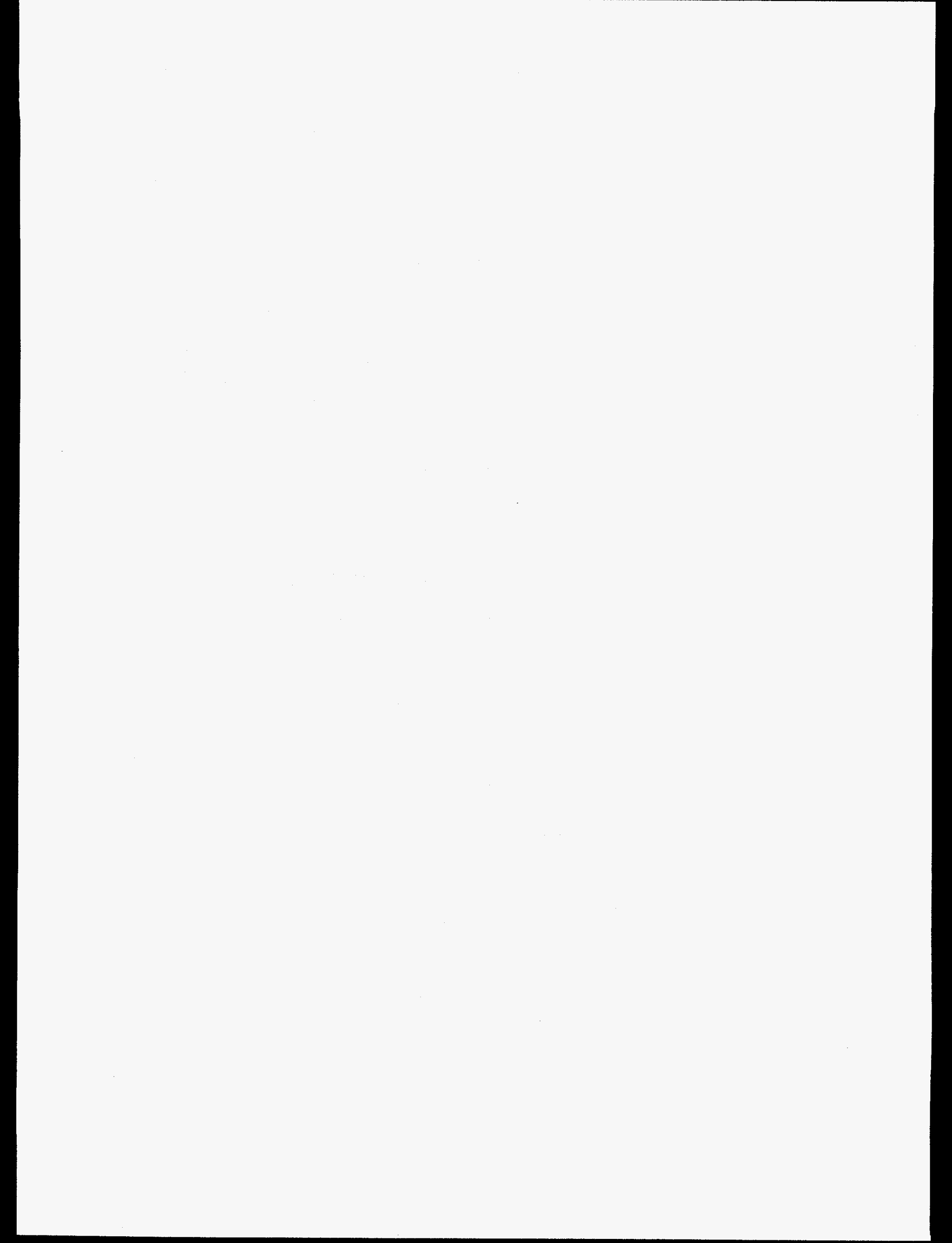


Appendix C

DRAWINGS

TBD

C-1

Appendix A, TM-13089 



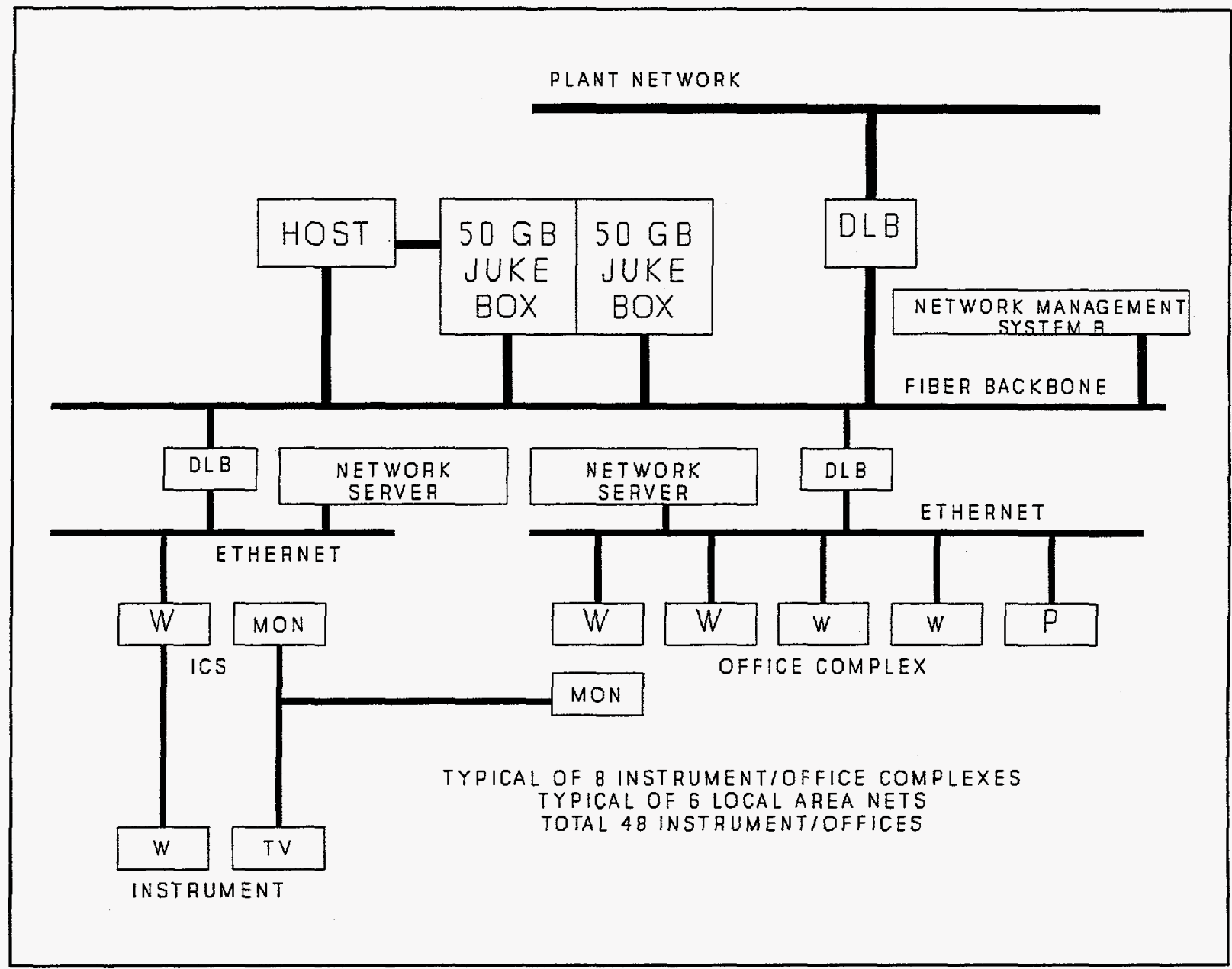

Fig. 1. Experiment Systems Computer Network Block Diagram (Only scattering instruments shown) 


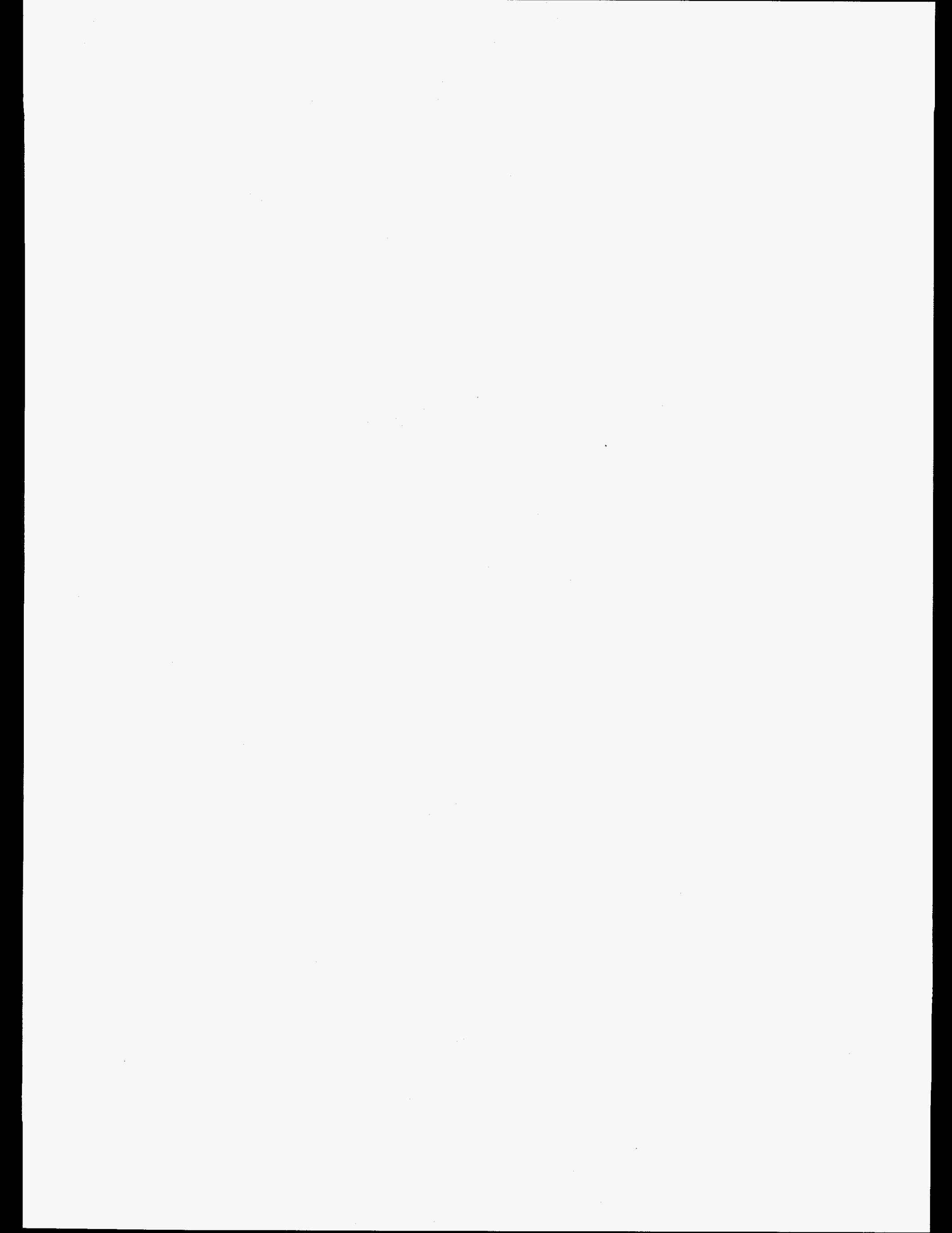


Appendix D

INSTRUMENT LIST

TBD

D-1 


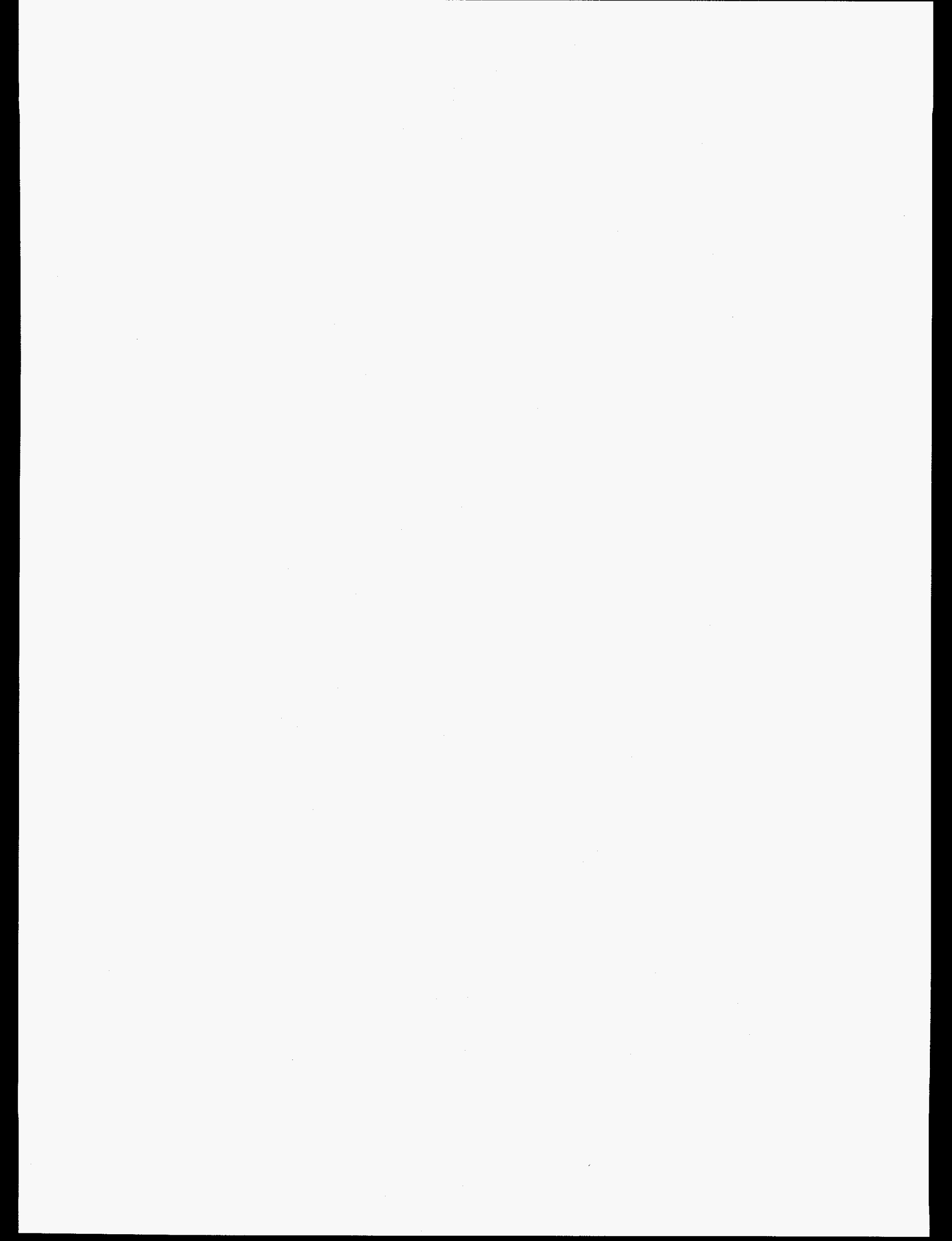


Appendix E

VALVE LIST

TBD

E-1

Appendix A, TM-13089 

Appendix F

EQUIPMENT LIST

TBD 


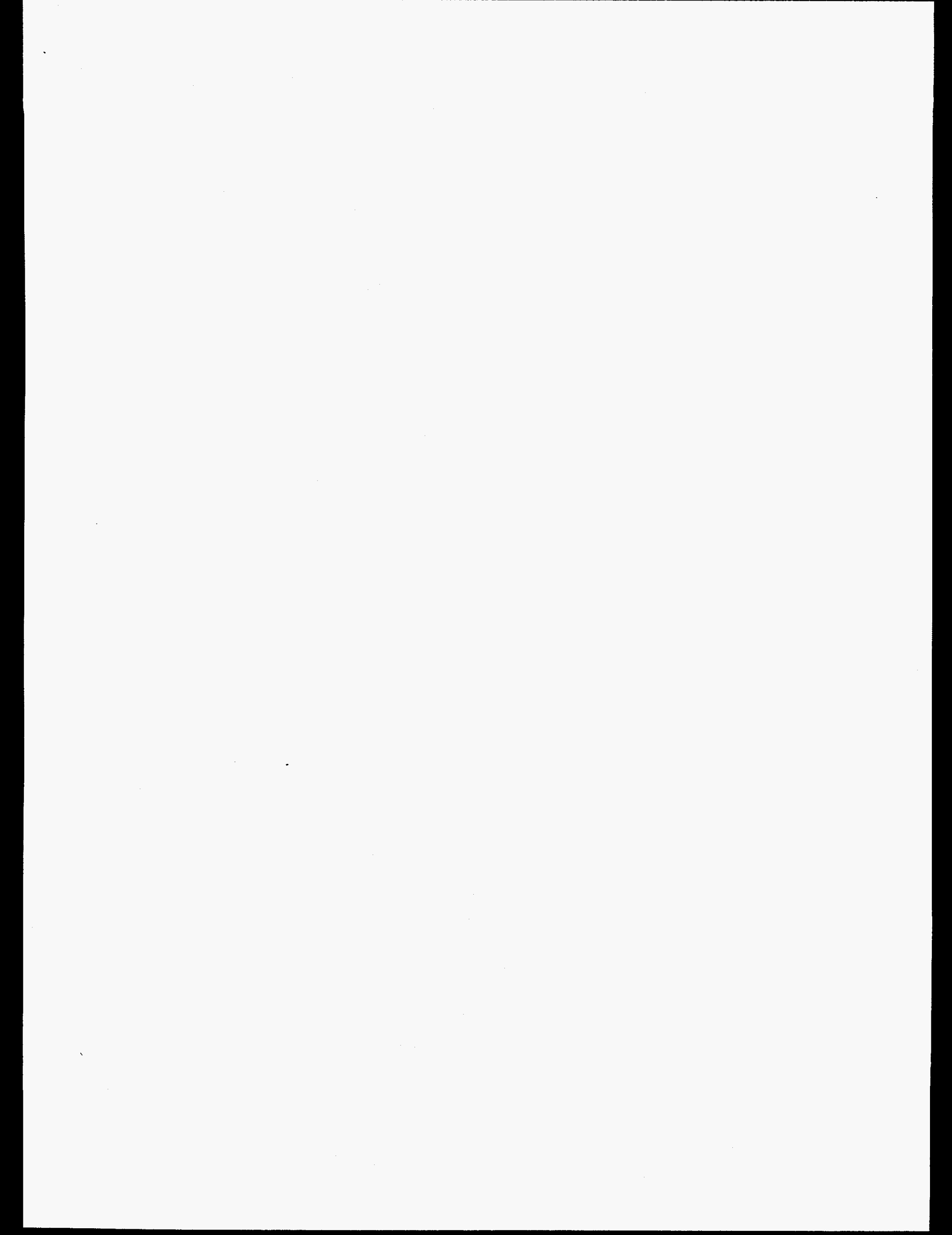


Appendix G

LINE LIST

TBD

G-1 


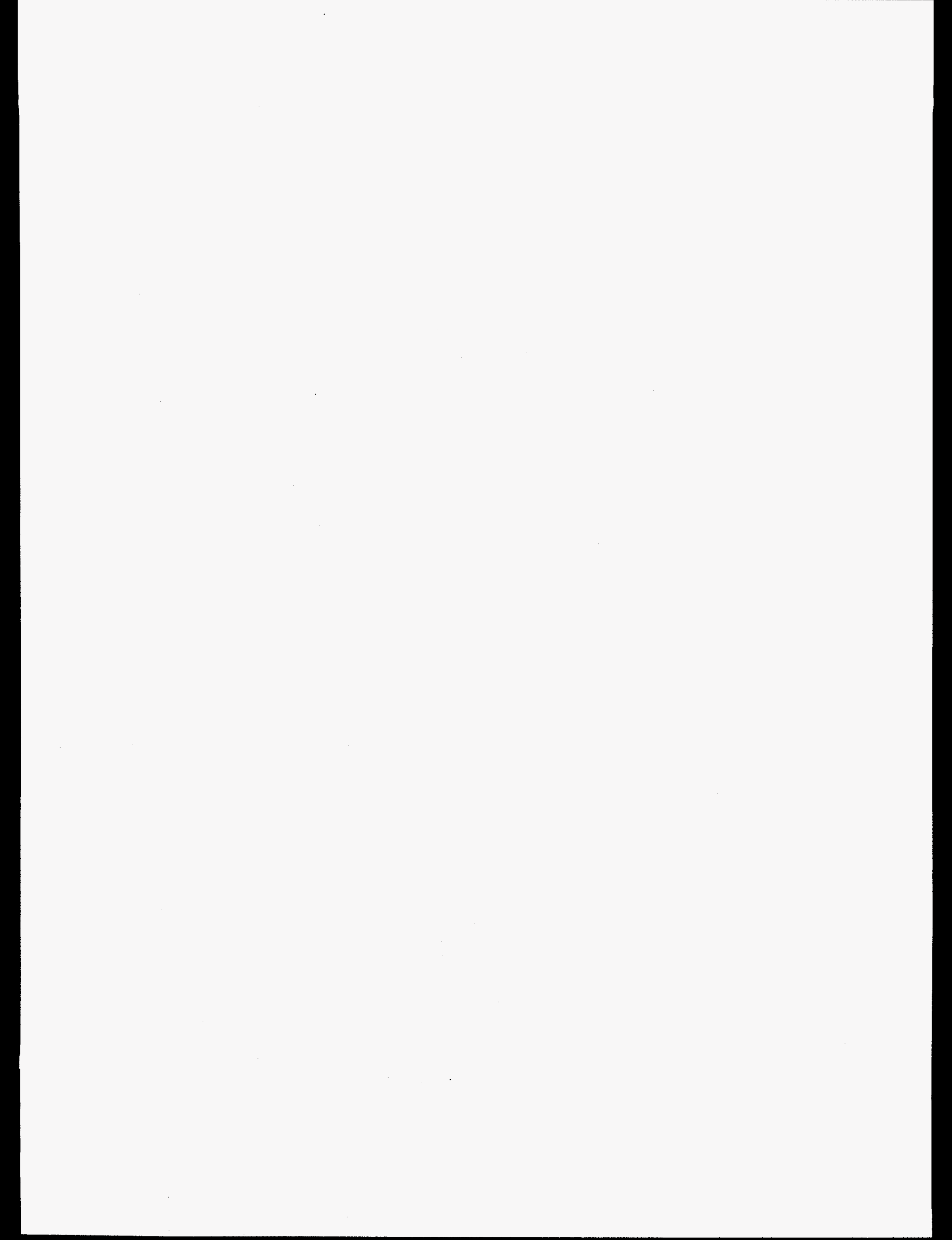


Appendix H

PRIMARY SYSTEM INTERFACES

TBD 


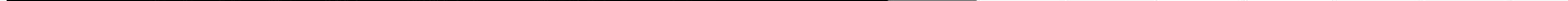


Appendix I

SECONDARY SYSTEM NTERFACES

TBD 

Appendix J

APPLICABLE STANDARDS, CODES, AND GUIDES 


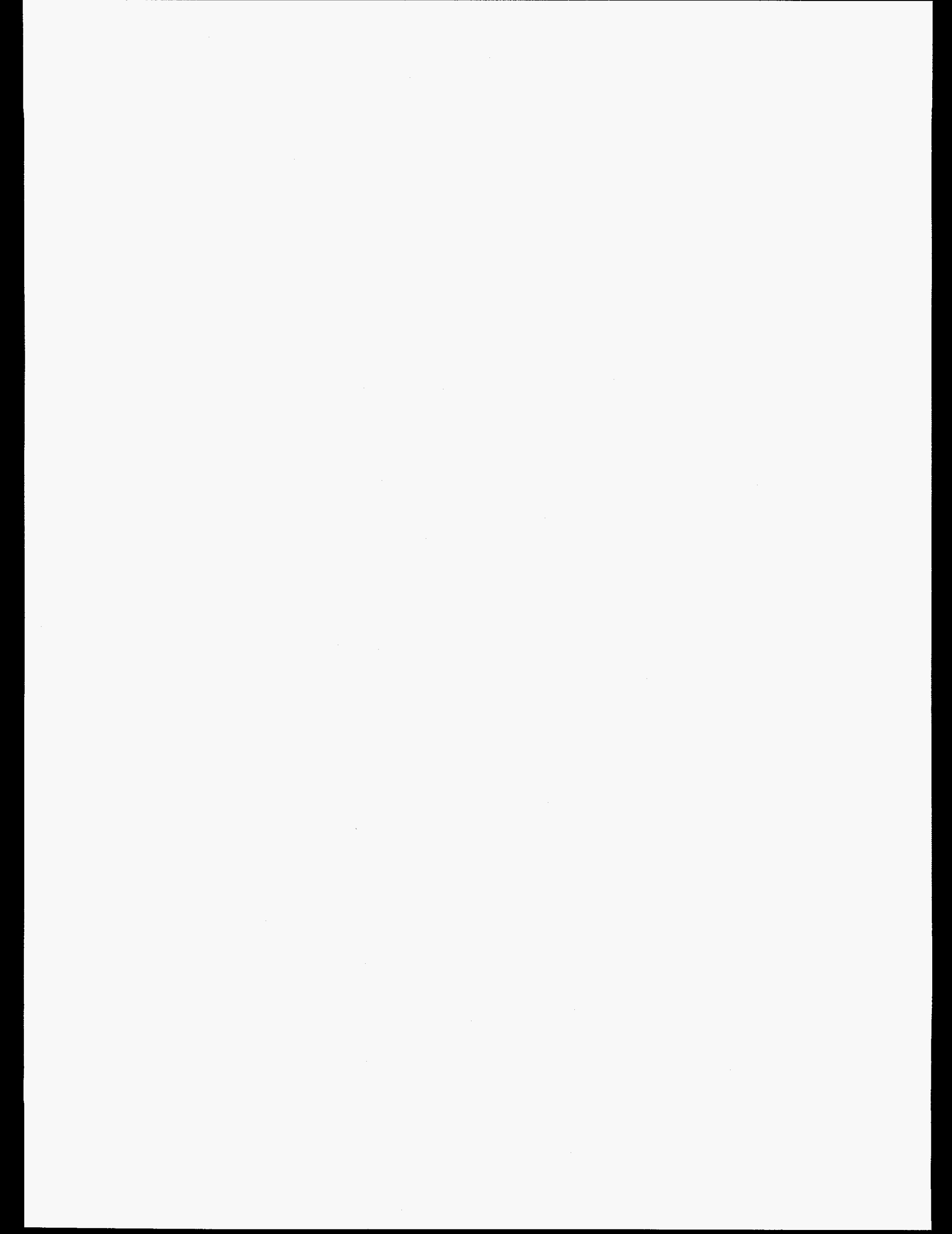


Document Number

Regulatory Documents

RG 1.8

RG 1.100

RG 1.11

RG 1.148

RG 1.28

RG 1.29

RG 1.30

RG 1.32

RG 1.33

RG 1.38

RG 1.73

RG 1.74

RG 4.1

RG 5.15

RG 1.57 (6.73)

NUREG/CR-0943

NUREG/CR-4892
Title

Qualification and Training of Personnel for Nuclear Power Plants

Seismic Qualification of Electric and Mechanical Equipment for Nuclear Power Plants

Instrument Lines Penetrating Reactor Containment

Functional Specification for Active Valve Assemblies in Systems Important to Safety in Nuclear Power Plants

Quality Assurance Program Requirements (Design and Construction)

Seismic Design Classification

Quality Assurance Requirements for the Installation, Inspection, and Testing of Instrumentation and Electric Equipment

Criteria for Safety-Related Electric Power Systems for Nuclear Power Plants

Quality Assurance Program Requirements (Operation)

Quality Assurance Requirements for Packaging, Shipping, Receiving, Storage, and Handling Items for Water-Cooled Nuclear Power Plants

Qualification Tests of Electric Valve Operators Installed Inside the Containment of Nuclear Power Plants

Quality Assurance Terms and Definitions

Programs for Monitoring Radioactivity in the Environs of Nuclear Power Plants

Security Seals for the Protection and Control of Special Nuclear Material

Design Limits and Loading Combinations for Primary Reactor Containment System Components

Threaded-Fastener Experience in Nuclear Power Plants

A Study of the Effects of Penetration Framing on Steel Containments Buckling Capacity 
Document Number

Nonregulatory Documents

ACI SP-84

ANSI B16.9

ANSI B16.11

ANSI B16.25

ANSI B16.34

ANSI B16.41

ANSI B16.5-1981

ANSI B16.9-1982

ANSI MC96.1

ANSI N45.2.5-1974

ANSI N278.1-1975 (R1984)

ANSI Y.32.16-1975

ANSI Y.32.2-1975

ASCE

ASME

ASME B31.8-1982

ASME OM-14

(Proposed)

ASME OM-6

(Proposed)

AWWA C500-71

ASME PTC 35

(DRAFT)

AWS D1.1-83
Title

Earthquake Effects on Reinforced Concrete Structures

Wrought Steel Butt Welding Fittings

Steel Fittings Steel Welding and Threaded

Butt Welding Ends - Pipe, Valves, Flanges, and Fittings

Steel Valves with Flanged and Butt Welding Ends

Functional Qualification Requirements for Power Operated Active Valve Assemblies for Nuclear Power Plants

Steel Pipe Flanges and Flanged Fittings

Building Services Piping

American National Standard for Temperature Measurement Thermocouples

Supplementary Quality Assurance Requirements for Installation, Inspection, and Testing of Structural Concrete and Structural Steel During the Construction Phase of Nuclear Power Plants

Self-operated and Power-operated Safety-related Valves Functional Specification Standard

Reference Designations for Electrical and Electronics Parts and (See ANSI/IEEE) Equipment (DOD Adopted)

Graphic Symbols for Electrical and Electronics Diagrams (Including Reference Designations Class Designation Letters)

Suggested Specification for Structures of Aluminum Alloys 6061-T6 and 6067-T6

ASME Boiler and Pressure Vessel Code

Gas Transmission and Distribution on Piping Systems

Requirements for Vibration Monitoring of Rotating Equipment

Requirements for Inservice Testing of Pumps in Light Water Cooled Nuclear Power Plants

Standard for Gate Valves, 3 in. Through 48 in., for Water and Other Liquids

ASME Code for Inservice Testing of Pumps in Nuclear Power Plants

Structural Welding Code-Steel 
Document Number

AWS D1.3-81

NEMA Standard

Publication IC56

NEMA ICS3-1978,

Part ICS3-304

NEMA Standard

Publication 250

\section{Title}

Specifications, Structural Welding Code-Sheet Steel

Enclosures for Industrial Control Systems

Industrial Controls and Systems

Enclosures for Electrical Equipment 


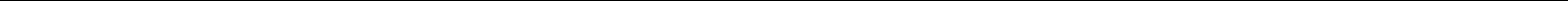


Appendix K

SYSTEM/COMPONENT SAFETY CLASSIFICATION AND STRUCTURAL DESIGN BASIS 

SDD 43: Materials Irradiation Facilities

\begin{tabular}{|c|c|c|c|c|c|c|c|}
\hline $\begin{array}{l}\text { System/ } \\
\text { Component }\end{array}$ & $\begin{array}{l}\text { Safety } \\
\text { Class }\end{array}$ & $\begin{array}{l}\text { Applicable } \\
\text { Construction } \\
\text { Code }\end{array}$ & $\begin{array}{l}\text { Seismic } \\
\text { Category }\end{array}$ & $\begin{array}{l}\text { Tornado } \\
\text { Category }\end{array}$ & $\begin{array}{c}\text { Design } \\
\text { Temperature } \\
{ }^{\circ} \mathrm{C}\left({ }^{\circ} \mathrm{F}\right)\end{array}$ & $\begin{array}{c}\text { Design } \\
\text { Pressure } \\
\mathrm{kPa} \text { (psig) }\end{array}$ & Notes \\
\hline
\end{tabular}

Thermal Nuclear Physics

Through-Tube Loading Station

NRS-1 ASME VIII

Class 1

I-L

Ultra-High Resolution $\gamma$-Spectroscopy

Polarized Nuclear Physics

Precision $\gamma$-ray Bolometer

NRS-1 ASME VIII

I-L

Weak Interaction Physics

Class 1

Parity Violation

General Nuclear Physics

Nuclear Structure

NRS-1 ASME VIII

I-L

Nuclear Spectroscopy

Class 1 

Appendix L

ELECTRICAL LOAD LIST

TBD

L-1

Apperdix A, TM-13089 


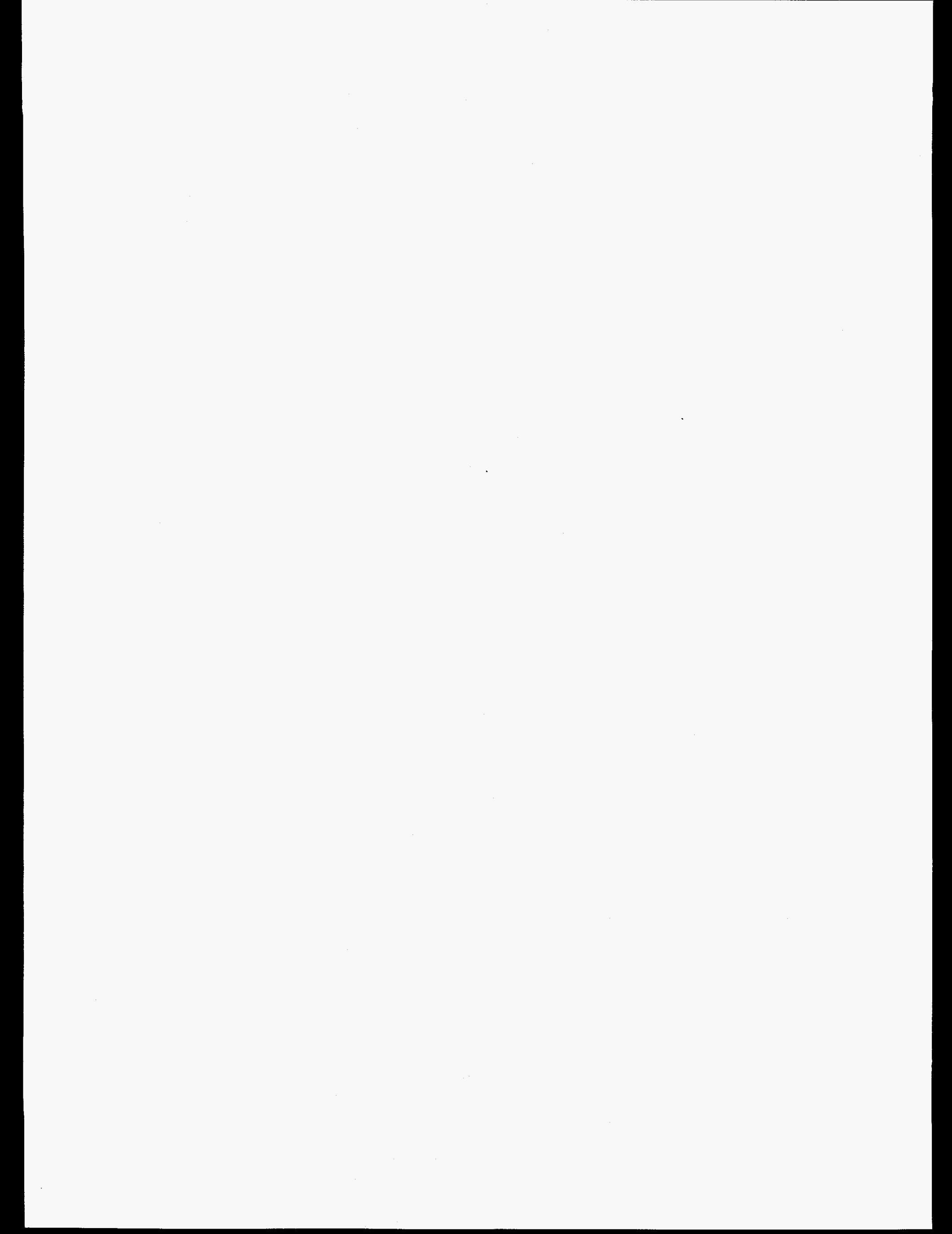


Appendix M

REQUIREMENTS TRACEABILITY INDEX

TBD 


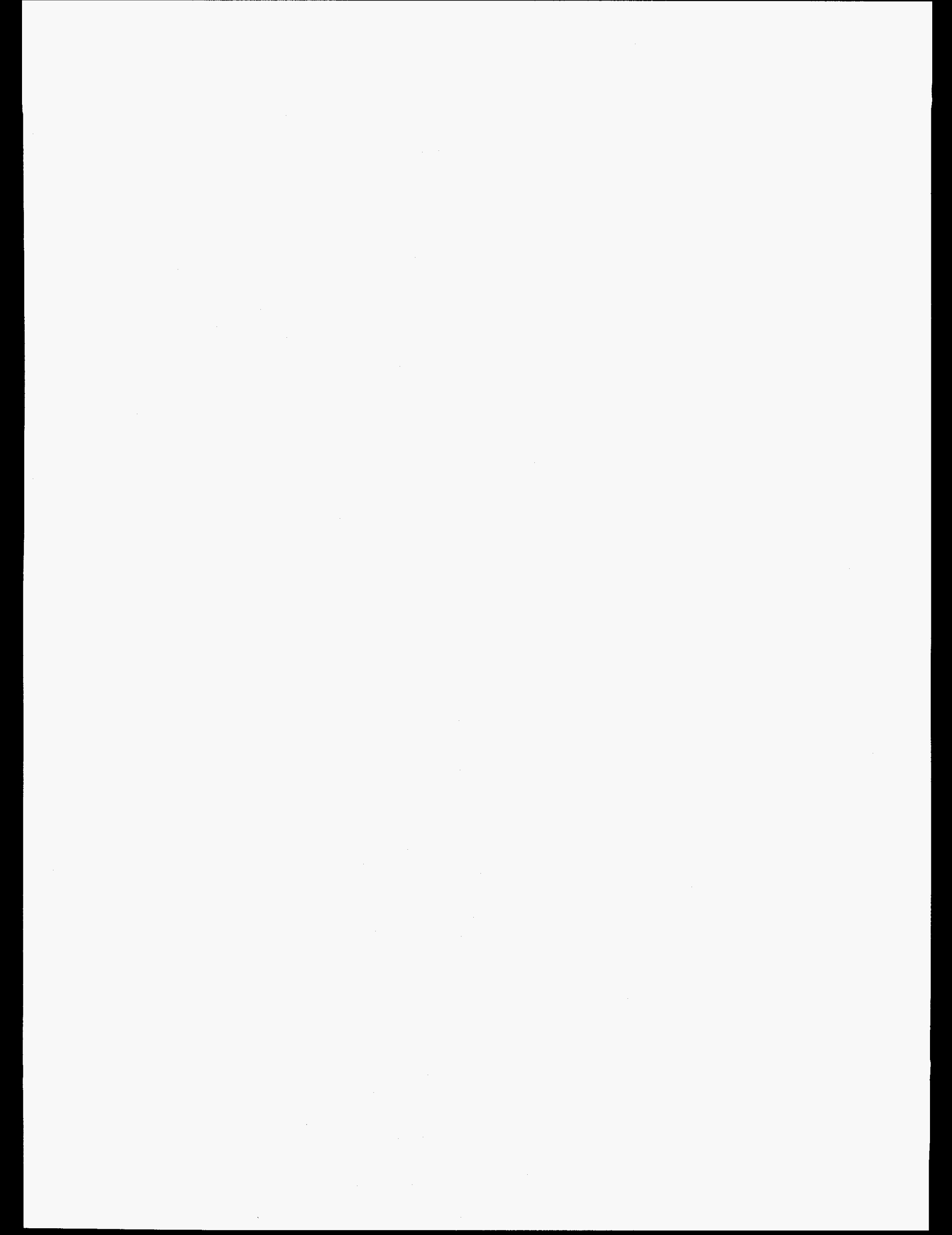


Appendix $\mathbf{N}$

CONFORMANCE WITH REGULATORY (NRC, DOE) REQUIREMENTS

TBD 
Appendix 0

HOLD/TBD RESPONSIBILITY-STATUS LIST

TBD

0-1 


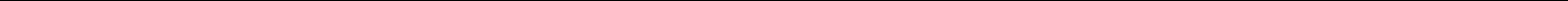


Appendix P

CONTAINMENT PENETRATION SCHEDULE

TBD 

Appendix B

COST AND PERSONNEL ESTIMATES 

Table B.1. Estimated instrument material costs and personnel requirements for nuclear and fundamental physics instruments at the ANS,

Version 1.2, March 1992

All costs in $\mathbf{k \$}$; all personnel estimates in person-months

Thermal Nuclear Physics

Through-tube loading station

Material Costs

Item

$\underline{\text { Cost }}$

Source Irradiation Assembly

500

Interface and remote handling

700

(Cost scaled from similar installation at ILL in 1985)

Computer and Interface

Total

(Contingency (50\%)

Personnel Estimates

Phase

Design and Planning

Time

Construction and Installation

Precision K-ray Spectroscopy

\section{Material Costs}

$\underline{\text { Item }}$

Crystal Gamma-Ray Spectrometer

Cost

(From instrument under construction at ILL)

(Contingency (10\%)

900

Pair formation spectrometer

(Estimate scaled from purchase at ILL in 1989)

(Contingency (20\%)

Associated electronic modules

(Estimate from current $E G \& G$ catalog)

Computer and Interface

Total

1025

(Contingency

106) 


\section{Personnel Estimates}

Phase

Design and Planning (Crystal Spectrometer)

Time

Design and Planning (Pair Spectrometer)

Design and Planning (Total)

Construction and Installation (Crystal Spectrometer)

Construction and Installation (Pair Spectrometer)

Construction and Installation (Total)

Polarized Nuclear Physics

Helium-Dilution Refrigerator (for y-ray Bolometer)

\section{Material Costs}

$\underline{\text { Item }}$

Dilution Refrigerator

Installation costs

(Costs scaled from installation of identical device

at ORNL (UNISOR) in 1985. This was purchased

from Oxford Instruments Ltd.)

Computer and Interface

Total

(Contingency (10\%)

Personnel Estimates

Phase

Design and Planning

Construction and Installation

\section{Neutron Polarizers}

\section{Material Costs}

$\underline{\text { Item }}$

Reflection type (4 off)

(Based on average cost of $k \$ 25$ per device constructed at ILL)

Neutron Polarizer ( ${ }^{3} \mathrm{He}$ type)

(Based on estimates from NIST study of such a device) 
Personnel Estimates

Phase

Design and Planning (Reflection Type)

Time

Design and Planning (He type)

Design and Planning (Total)

Construction and Installation (Reflection type)

Construction and Installation (He type)

Construction and Installation (Total)

$\frac{6}{30}$

Neutron Interferometer

Material Costs

$\underline{\text { Item }}$

Neutron Interferometer

(Based on cost of device recently constructed at NIST,

includes construction and installation)

(Contingency (20\%)

Personnel Estimates

Phase

Time

Design and Planning

36

Construction and Installation

(Included in Material Costs)

General Nuclear Physics

General Detector Pool

Material Costs

$\underline{\text { Item }}$

Cost

Germanium gamma-ray detectors (10 off)

(Cost per detector of $k \$ 46.5$ from current $E G \& G$

catalog for $50 \%$ efficient $n$-type detector with

cryostat/dewar)

(Contingency (5\%)

Compton Suppression Shields (6 off)

(Estimate from Harshaw/Filtrol Ltd. of

approximately $k \$ 50$ per shield, exact cost to

depend on design geometry)

(Contingency (20\%) 
Fast Timing Barium Fluoride Detectors (4 off)

(Cost per detector of $k \$ 4$ from recent purchases

for similar system at ORNL (UNISOR))

(Contingency (10\%)

Particle Detectors

(Price from current EG\&G catalog based on two

each of alpha (k\$2), beta (k\$11) and neutron (k\$23)

detectors)

(Contingency (10\%)

Detector Electronics

(Estimate from current $E G \& G$ catalog; based on maximum need if all above detectors in use simultaneously)

(Contingency (20\%)

Flexible detector Mounting Framework

(Contingency (50\%)

Computer and Interface (3 off)

Total

(Contingency

Personnel Estimates

Phase

Time

Design and Planning

Construction and Installation

\section{High Resolution Sector Magnet Electron Spectrometer}

Material Costs

Item

$\underline{\text { Cost }}$

Electron Spectrometer

**300

(Contingency (100\%)

Personnel Estimates

Phase

Design and Planning

Time

Construction and Installation

$* * 12$

$* * 12$ 


\section{On Line Isotope Separator}

\section{Material Costs}

Item

Ion Source Assembly for Isotope Separator

$\underline{\text { Cost }}$

(From estimates of upgrade of TRISTAN at Brookhoven

National Lab.)

(Contingency (50\%)

Beam Line and Ion Transport Mechanism

(From estimates of upgrade of TRISTAN at Brookhoven

National Lab.)

(Contingency (50\%)

Isotope Separator

$* 1,000$

(Contingency (50\%)

Computer and Interface

Total

(Contingency

Personnel Estimates

Phase

Time

Design and Planning (Ion Source)

Design and Planning (Beam Line)

Design and Planning (Separator)

Design and Planning (Total)

Construction and Installation (Ion Source)

Construction and Installation (Beam Line)

Construction and Installation (Separator)

Construction and Installation (Total)

Notes: ${ }^{*}$ To be verified, ${ }^{* *}$ Estimated. 


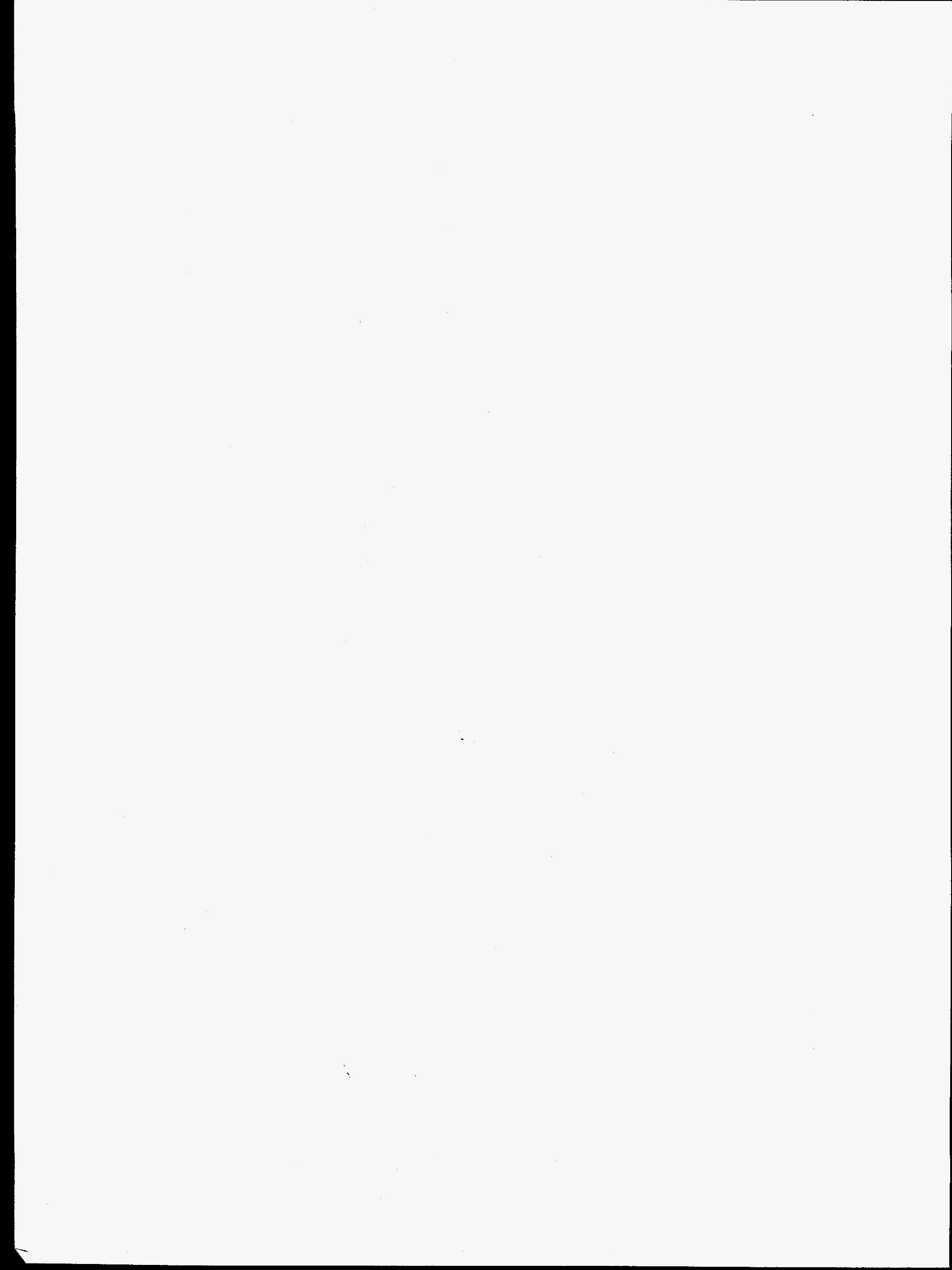


Appendix C

CONVENTIONAL ION SOURCE TYPES 



\section{C.1 INTRODUCTION}

Several different types of conventional ion sources are discussed briefly. Each has its own merits in terms of which elements are easily delivered. If this approach is chosen for the ANS ISOL facility, it is likely that some or all of these types will be needed.

\section{C.2 SURFACE-IONIZATION ION SOURCE}

This type of source relies on the fact that some atomic species ionize readily when they come into contact with a hot surface. The usual choice for the ionizing surface material is rhenium or tantalum. The incoming neutron beam interacts with the fissile material inside the ion source. The whole assembly is heated to $>2000^{\circ} \mathrm{C}$ using direct heating and electron bombardment, facilitating the diffusion of the fission products through the target matrix material (usually graphite). The fission products diffuse to the ionizing surface, from which, once ionized, they can be easily extracted.

Such sources have been fully developed and have lifetimes of $>2500 \mathrm{~h}{ }^{1}$ However, surface ionization is suitable only for producing beams of $\mathrm{Rb}, \mathrm{Sr}, \mathrm{Y}, \mathrm{Cs}, \mathrm{Ce}$, and $\mathrm{Pr}$.

\section{C.3 HIGH-TEMPERA TURE PLASMA ION SOURCE}

In this type of ion source, fission products are ionized by collisions with the particles in a high-temperature plasma discharge cloud. Again high temperatures $\left(>2500^{\circ} \mathrm{C}\right)$ encourage the rapid diffusion of fission products, which will eventually reach a region in which a continuous discharge takes place. In some designs the target is located at, and is actually part of, the discharge cathode, making for a higher efficiency. A source of this type has been tested at the TRISTAN separator at Brookhaven National Laboratory ${ }^{1}$ and has been shown to give an order of magnitude higher yield for certain elements than the more conventional FEBIAD ion source.

In addition, the plasma ion source allows the extraction of a much wider range of elemental beams, such as $\mathrm{Cu}, \mathrm{Zn}, \mathrm{Ga}, \mathrm{Ge}, \mathrm{As}, \mathrm{Se}, \mathrm{Br}, \mathrm{Kr}, \mathrm{Rb}, \mathrm{Sr}, \mathrm{Y}, \mathrm{Ag}, \mathrm{Cd}, \mathrm{In}, \mathrm{Sn}, \mathrm{Sb}, \mathrm{Te}, \mathrm{I}, \mathrm{Xe}, \mathrm{Cs}, \mathrm{Ba}, \mathrm{Ce}$, and Pr. It is this method of ionization that is used in the helium-jet ion source described in Sect. 3.3.4 of this report.

\section{C.4 THE BI-MODE ION SOURCE}

This unconventional bi-mode ion source was developed for use at the isotope separator in Studsvik (OSIRIS) $)^{2,3}$ and can be operated in either surface-ionization or plasma-discharge mode, depending on the configuration of the potentials between various elements of the ion source. Using this design, a virtually complete scan over the whole range of uranium fission products is possible with overall efficiencies ranging from 0.03 to $30 \%$, depending on the chemical species involved. This source has also been used to study oxide and carbide molecular ions formed by the fission products inside the ion source. 


\section{C.5 THE FEBIAD ION-SOURCE}

A modification of this standard ion source design ${ }^{4}$ has been developed at the TRISTAN separator. These modifications have allowed the target area to make use of the full size of the neutron beam and have also extended the lifetime of the ion source to up to $1500 \mathrm{~h}$. This type of source allows the extraction of beams of $\mathrm{Cu}, \mathrm{Zn}, \mathrm{Ga}, \mathrm{Ge}, \mathrm{Se}, \mathrm{Br}, \mathrm{Kr}, \mathrm{Rb}, \mathrm{Ag}, \mathrm{Cd}, \mathrm{In}, \mathrm{Sn}, \mathrm{Sb}, \mathrm{Te}, \mathrm{I}$, and $\mathrm{Xe}$.

\section{C.6 OTHER DESIGNS}

Several other types of ion source currently under different stages of development show promise for use at the ANS facility. Included among these are attempts to create efficient negative ion sources, attempts to reduce dwell times using laser-induced desorption, and, showing particular promise, plasma-type ion sources involving confinement using electron cyclotron resonance effects. Progress in ion source development is difficult to predict, and it is entirely possible that other designs may be forthcoming in time for incorporation the final planning for the ANS ISOL facility.

\section{C.7 REFERENCES}

1. M. Shmid et al., "A Long-Lived Surface-Ionization Ion Source," Nucl. Instrum. Methods 211, 287 (1983).

2. L. Jacobsson et al., "An Unconventional Bi-Mode Ion Source (ANUBIS) for the OSIRIS Facility," Nucl. Instrum. Methods B26, 223 (1987).

3. B. Fogelberg et al., "Efficiency and Delay of the Integrated Target-Ion Source of OSIRIS," Nucl. Instrum. Methods B70, 137 (1992).

4. R. Kirchner et al., "Ion Source Development for the On-Line Isotope Separator at GSI," Nucl. Instrum. Methods B70, 56 (1992). 


\section{INTERNAL DISTRIBUTION}

1. B. R. Appleton

2-4. J. G. Arterburn

5. J. B. Ball

6-10. S. J. Ball

11. F. E. Bertrand

12-16. J. H. Campbell

17. R. L. Johnson

18-20. T. J. McManamy

21-23. F. J. Peretz

24-28. S. Raman
29. D. L. Selby

30-34. J. K. Thacker

35. C. D. West

36. ORNL Patent Office

37-38. Central Research Library

Document Reference Section

39. Y-12 Technical Library

40-41. Laboratory Records Dept.

42. Laboratory Records, RC

\section{EXTERNAL DISTRIBUTION}

43-45. H. Faust, Institut Laue-Langevin, Grenoble, France

46-48. A. E. Piotrowski, Soltan Institute for Nuclear Studies, OS-400, Swierk, Poland

49-51. S. J. Robinson, Physics Department, Tennessee Technological University, Cookeville, TN 38505

52. U.S. Department of Energy, ANS Project Office, Oak Ridge Operations Office, FEDC, MS-8218, P.O. Box 2009, Oak Ridge, TN 37831-8218

53-54. Office of Scientific and Technical Information, P.O. Box 63, Oak Ridge, TN 37831 
